\title{
Numerical simulations of non-spherical bubble collapse
}

With applications to shockwave lithotripsy

Thesis by

Eric Johnsen

In Partial Fulfillment of the Requirements for the Degree of

Doctor of Philosophy

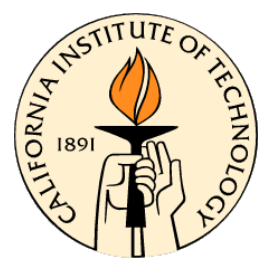

California Institute of Technology

Pasadena, California

2007

(Defended November 29, 2007) 
In the memory of Kjell Johnsen (1921-2007). 
(C) 2007

Eric Johnsen

All Rights Reserved 


\section{Acknowledgements}

Je tremble de n'avoir écrit qu'un soupir, quand je crois avoir noté une vérité.

Stendhal

First and foremost, I wish to express my genuine gratitude to my advisor, Professor Tim Colonius. His guidance over the course of my stay at Caltech has had a profound influence on my development, both academic and personal. Tim provided me with the freedom to pursue my own ideas while still offering valuable suggestions and encouragement when needed. I consider myself fortunate to have had a mentor who dedicates so much time and attention to his students and hope to one day be able to emulate his example.

I thank Professors John Brady, Chris Brennen and Joe Shepherd who, in addition to forming my thesis committee, have contributed many useful comments to this work. Their insightful suggestions have steered my research towards very interesting directions. I also wish to acknowledge the Caltech faculty who have taught me many interesting lessons in the classroom and in life.

I am grateful to Andy Evan and the PPG group for providing the opportunity to cast my research in such an interesting field as shockwave lithotripsy. I was able to appreciate how a collection of individuals with very diverse backgrounds manages to carry out such a successful collaboration in congeniality. Many thanks go to Mike Bailey, Robin Cleveland, Larry Crum, Jon Freund, Raj Honda, Wayne

Kreider, Jim McAteer, Yuri Pischchalnikov, Oleg Sapozhnikov, and Jim Williams, for numerous discussions on cavitation, wave propagation and kidney stones.

One of the main reasons I managed to keep my sanity over the course of my Ph.D. is the fantastic atmosphere that resides in the Computational Flow Physics Group. Previous (Al Preston, Hongyu Ran, Michel Tanguay) and contemporary (Keita Ando, Guillaume Brès, Rick Burnes, Vedran Coralic, Jen Franck, Kristjan Gudmundsson, Won Tae Joe, Jeff Krimmel, and Sam Taira) members have all provided invaluable help, ranging from deep discussions on fluid mechanics and CFD to lunches/drinks at the Ath, from programming tricks and network administration 
to comic relief, from Cavitation group meetings (thanks to occasional attendees Rob Hagmeijer and Arjen Koop) to wings at Gus's - oftentimes combining several of these items! I also wish to acknowledge the dwellers of the Thomas building, who form an enjoyable and supportive community. In particular, I thank SOPS for the organization of social hours, BBQs, and intramural soccer and basketball teams, and the wonderful staff (in particular Lynn Burgess, Cheryl Geer and Chris Silva) for always being available for assistance.

My good friends at Caltech, including Corinne Ladous, Stuart Laurence, Angel Ruiz-Angulo, Pavlin Savov, and Fuling Yang, deserve special mention for their company and insights into all sorts of matters, academic and leisurely. I also express my heartfelt gratitude to my parents and sisters for their overseas support, encouragement and understanding, and to my grandfather, Kjell Johnsen, whose interest in my work led to many cherished discussions on physics.

Caltech is a truly special place, and I am confident that I will forever cherish the few years I was fortunate to spend here. I was able to learn from world-class scientists and engineers, work with the brightest colleagues, and meet such interesting people, some of whom will remain lifelong friends. But what I did not anticipate (and for which I am most grateful) was to encounter my soulmate and true love, Julie Biteen, in the very confines of the campus. Throughout my studies at Caltech, she stood by my side, sharing my joys and my fears, my successes and my failures. Without her understanding, love and support, I doubt my ability to have sucessfully completed this thesis.

This work was supported by NIH Grant PO1 DK043881 and ONR Grant N00014-06-1-0730. 


\title{
Numerical simulations of non-spherical bubble collapse \\ With applications to shockwave lithotripsy
}

by

\author{
Eric Johnsen
}

\begin{abstract}
Shockwave lithotripsy (SWL) is a non-invasive medical procedure in which shockwaves are focused on kidney stones in an attempt to break them. Because the stones are usually immersed in liquid, cavitation occurs during the process. However, the stone comminution mechanisms and the bubble dynamics of SWL are not fully understood. In the present thesis, numerical simulations are employed to study axisymmetric Rayleigh collapse and shock-induced collapse of a single gas bubble in a free field and near a wall. A high-order accurate, quasi-conservative, shock- and interface-capturing scheme is developed to solve the multicomponent Euler equations.

The primary contributions of the present work are the development of a new numerical framework to study compressible multicomponent flows, the characterization of the dynamics of non-spherical bubble collapse, and quantitative measurements of wall pressures generated by bubble collapse. Because of asymmetries in the flow field, a re-entrant jet develops and generates a large water-hammer pressure upon impact onto the distal side. Jet properties are calculated and, as an indication of potential damage, wall pressures are measured; pressures on the order of $1 \mathrm{GPa}$ are achieved locally. In shock-induced collapse, the wall pressure is amplified by the presence of bubbles within several initial radii from the wall. Thus, the pressure generated by the bubble collapse is larger than the incoming shock. The results extended to SWL show that shock-induced collapse has tremendous potential for damage along the stone surface. Furthermore, the simulations are coupled to an elastic wave propagation code to show that bubble collapse may cause damage within kidney stones as well.
\end{abstract}




\section{Contents}

List of Figures $\quad \mathrm{xx}$

List of Tables $\quad$ xxi

List of Publications $\quad$ xxiii

Nomenclature $\quad$ xxv

1 Bubble collapse and cavitation damage 1

1.1 The occurrence of bubbles in flows of liquids . . . . . . . . . . . . 1

1.2 Cavitation damage . . . . . . . . . . . . . . . . 2

1.2.1 The violence of bubble collapse . . . . . . . . . . . 2

1.2.2 Undesirable cavitation damage . . . . . . . . . . . . 3

1.2.3 Desirable cavitation damage . . . . . . . . . . . . 6

1.3 Single-bubble dynamics . . . . . . . . . . . . . . . . 11

1.3.1 Theoretical and experimental studies . . . . . . . . . . 11

1.3.2 Numerical simulations . . . . . . . . . . . . . . . 15

1.4 Motivation, accomplishments and overview . . . . . . . 17

2 Physical model $\quad 21$

2.1 Problem description . . . . . . . . . . . . . . . . . 21

2.1.1 Single-bubble collapse in shockwave lithotripsy . . . . . . 21

2.1.2 Domain . . . . . . . . . . . . . . . . . 23

2.2 Equations of motion . . . . . . . . . . . . . . . 25

2.2.1 Compressible multicomponent flows . . . . . . . . . 25 
2.2 .2 The Euler equations . . . . . . . . . . . . . . . 27

2.2 .3 The stiffened equation of state . . . . . . . . . . . 29

2.2 .4 Advection equations . . . . . . . . . . . . . . . 29

2.2.5 Non-dimensionalization and physical parameters . . . . . 30

2.3 Summary of the physical model . . . . . . . . . . . . . . . 32

3 Numerical method $\quad 35$

3.1 Numerical simulations of interfaces and shockwaves . . . . . . . 35

3.1 .1 Shock capturing . . . . . . . . . . . . . 36

3.1 .2 Interface tracking and capturing . . . . . . . . . 36

3.1.3 Present methodology . . . . . . . . . . . . . 37

3.2 Time-marching scheme . . . . . . . . . . . . . . . . 38

3.3 Shock-capturing scheme . . . . . . . . . . . . . . . 39

3.3.1 Finite volume vs. conservative finite difference . . . . . . 39

3.3.2 Finite volume WENO reconstruction . . . . . . . . . . . 40

3.3.3 The HLLC approximate Riemann solver . . . . . . . . . . . 46

3.4 Interface-capturing scheme . . . . . . . . . . . . . . . . . . . 49

3.4.1 Occurrence of oscillations at isolated interfaces . . . . . . 49

3.4.2 Reconstruction of the primitive variables . . . . . . . . . 50

3.4.3 Oscillation-free advection of an isolated interface . . . . . 52

3.5 Boundary conditions . . . . . . . . . . . . . . . 53

3.5.1 Reflecting boundary conditions . . . . . . . . . . 53

3.5.2 Non-reflecting boundary conditions . . . . . . . . . . . 54

3.6 Verification .......................... 56

3.6 .1 Convergence analysis . . . . . . . . . . . 56

3.6.2 Isolated interface advection problem . . . . . . . . . . 58

3.6.3 Shock-tube problems . . . . . . . . . . . . . . 60

3.6.4 Shock-bubble interactions . . . . . . . . . . . 65

3.7 Extension of the numerical methodology . . . . . . . . . . 71

3.7.1 Finite volume equations in cylindrical coordinates . . . . 71 
3.7.2 Boundary conditions at $r=0 \ldots \ldots \ldots 72$

3.7.3 Computational domain . . . . . . . . . . . . 73

3.7 .4 Initial conditions . . . . . . . . . . . . . . 75

3.8 Limitations of the numerical scheme . . . . . . . . . . . . 76

3.8.1 Inviscid simulations . . . . . . . . . . . . . 76

3.8.2 Resolution. . . . . . . . . . . . . . . 79

3.9 Summary of the numerical method . . . . . . . . . . . 81

4 Dynamics of non-spherical bubble collapse $\quad 83$

4.1 Preamble: spherical bubble dynamics . . . . . . . . . . . . . . 83

4.1.1 Rayleigh-Plesset equation . . . . . . . . . . . . 83

4.1.2 Velocity and pressure fields in the liquid . . . . . . . . 88

4.1 .3 Energy loss by radiation . . . . . . . . . . . . . . 91

4.1.4 Non-spherical perturbations . . . . . . . . . . . . . . 93

4.2 Flow visualization . . . . . . . . . . . . . . . . . 94

4.2.1 Free-field collapse . . . . . . . . . . . . . . . . . . 94

4.2 .2 Collapse near a wall . . . . . . . . . . . . . . . 97

4.3 Dynamics of collapse . . . . . . . . . . . . . . . . . . 101

4.3.1 Time scales in bubble collapse . . . . . . . . . . . . . 101

4.3.2 Initial motion of the interface . . . . . . . . . . . 103

4.3 .3 Interfacial instabilities . . . . . . . . . . . . . 106

4.3.4 Measure of non-sphericity . . . . . . . . . . . . . . . 108

4.3.5 Maximum jet velocity . . . . . . . . . . . . . . . 109

4.3 .6 Jet size . . . . . . . . . . . . . . . . . 113

4.3 .7 Collapse time . . . . . . . . . . . . . . 115

4.3.8 Displacement of the bubble centroid . . . . . . . . . . . 119

4.3 .9 Bubble circulation . . . . . . . . . . . . . . . . 121

4.4 Jet formation mechanism in shock-induced collapse . . . . . . . . 125

4.4.1 Flow induced by the passage of the shockwave . . . . . 126

4.4 .2 Non-spherical perturbations . . . . . . . . . . . . . . . 128 
4.4.3 Flow induced by baroclinic vorticity . . . . . . . . . . . 129

4.4.4 Transient nature of shock propagation . . . . . . . . . . 131

4.4.5 Rayleigh collapse near a wall . . . . . . . . . . . . . . 135

4.5 Damage potential of single-bubble collapse . . . . . . . . . . . . 137

4.5.1 Shock emission . . . . . . . . . . . . . . . . 138

4.5.2 Water-hammer pressure . . . . . . . . . . . 146

4.5 .3 Radiated energy . . . . . . . . . . . . . . . 150

4.5.4 Wall pressure . . . . . . . . . . . . . . 153

4.5.5 Dependence on the shock amplitude . . . . . . . . . . . 160

4.5 .6 Shielding . . . . . . . . . . . . . . . . 162

4.6 Summary of the bubble collapse results . . . . . . . . . . 163

5 Single-bubble collapse in shockwave lithotripsy 165

5.1 Modeling of the lithotripter pulse . . . . . . . . . . . . 165

5.2 Shock-induced collapse in SWL . . . . . . . . . . . . . . . 167

5.2.1 General observations of shock-induced collapse . . . . . . 168

5.2.2 Damage potential of shock-induced collapse in SWL . . . . 172

5.2.3 Rayleigh collapse in SWL . . . . . . . . . . . . . . . . 179

5.3 Stone comminution . . . . . . . . . . . . . . . . . . . . . 181

5.3 .1 Spallation and cavitation . . . . . . . . . . 181

5.3.2 A comminution mechanism for medium-sized stones . . . . 182

5.4 Summary of the SWL results . . . . . . . . . . . . . 185

6 Concluding remarks $\quad 187$

6.1 Summary and conclusions . . . . . . . . . . . . . . . 187

6.2 Suggestions for future work . . . . . . . . . . . . . . 190

6.2.1 Physical model . . . . . . . . . . . . . . . . . . . . . . 191

6.2 .2 Numerical method . . . . . . . . . . . . . . . . . . . 191

6.2.3 Extension to related problems . . . . . . . . . . . . . 192 
A The stiffened equation of state

A.1 Definitions . . . . . . . . . . . . . . . 195

A.1.1 Isentropic flow . . . . . . . . . . . . . . . 195

A.1.2 Thermodynamic quantities . . . . . . . . . . 196

A.1.3 Riemann invariants . . . . . . . . . . . . . . . 197

A.2 Rankine-Hugoniot relations . . . . . . . . . . . . . . . . 198

A.2.1 Determination of $\gamma$ and $P_{\infty} \ldots \ldots . \ldots 200$

A.3 Expansion waves . . . . . . . . . . . . . . . . 201

B WENO reconstruction on non-uniform grids 203

B.1 WENO polynomials . . . . . . . . . . . . . . . 203

B.2 Polynomial values at cell edges . . . . . . . . . . . . . . . 204

B.3 Ideal weights . . . . . . . . . . . . . . . 205

B.4 Smoothness indicators . . . . . . . . . . . . 206

$\begin{array}{ll}\text { C Post-processing } & 209\end{array}$

C.1 Interface location . . . . . . . . . . . . . . . . . . . 209

C.2 Bubble properties. . . . . . . . . . . . . . 209

C.2.1 Bubble volume . . . . . . . . . . . . . . . . . 209

C.2.2 Bubble position . . . . . . . . . . . . . . . . . . 210

C.2.3 Bubble pressure . . . . . . . . . . . . . . . 210

C.2.4 Bubble non-sphericity . . . . . . . . . . . . . 210

C.3 Circulation . . . . . . . . . . . . . . . . 211

C.4 Jet properties . . . . . . . . . . . . . . . . . . . . . 212

C.5 Numerical Schlieren contours . . . . . . . . . . . . . . . 212

$\begin{array}{lr}\text { Bibliography } & 213\end{array}$ 


\section{List of Figures}

1.1 Cavitation damage in the spillway tunnel of the Yellowtail Dam . . 5

1.2 Schematic of an underwater explosion . . . . . . . . . . 6

1.3 Schematic of the set-up for SWL using an electrohydraulic lithotripter 8

1.4 Pressure profile in the focal region of a lithotripter . . . . . . . 9

1.5 Spallation and cavitation damage caused to artificial kidney stones 10

1.6 Collapse of a laser-generated cavitation bubble near a solid surface 14

1.7 Shock-induced collapse of a laser-generated cavitation bubble in a free-field . . . . . . . . . . . . . . . . . . 15

2.1 Typical bubble cycle in SWL . . . . . . . . . . . . . . 22

2.2 Schematic of the problem geometry . . . . . . . . . . . . . 24

2.3 Solution to the Rayleigh-Plesset equation for one period . . . . . . 27

3.1 Schematic of reflecting boundary conditions on a Cartesian grid. . 53

$3.2 L_{1}$ and $L_{\infty}$ errors in density for a smooth acoustics problem $\left(\epsilon=10^{-4}\right) 58$

$3.3 L_{1}$ and $L_{\infty}$ errors in density for a smooth acoustics problem $\left(\epsilon=10^{-1}\right) 59$

3.4 Advection of a material interface for the finite difference, fully conservative finite volume, and quasi-conservative finite volume schemes 60

3.5 Advection of a material interface using the present scheme . . . . . 61

3.6 Modified Sod problem using the present scheme . . . . . . . . . 62

3.7 Gas-liquid Riemann problem using the present scheme . . . . . . . 63

3.8 Shock-interface interaction using the present scheme . . . . . . . 64

3.9 One-dimensional shock-bubble interaction using the present scheme 66 
3.10 One-dimensional shock-bubble interaction, using the quasi-conservative FV scheme ..................... 67

3.11 Interaction between a Mach 1.22 shockwave in air and a He bubble using the present scheme . . . . . . . . . . . . . 68

3.12 Pressure profiles in shock-bubble interaction . . . . . . . . . . . . . 69

3.13 Interaction between a Mach 1.22 shockwave in air and a He bubble using the quasi-conservative scheme . . . . . . . . . 70

3.14 Schematic of the grid points near the centerline . . . . . . . . 72

3.15 Grid-stretching function . . . . . . . . . . . . . . . . . . 74

3.16 Computational grid used for Rayleigh collapse . . . . . . . . . . . . 75

3.17 Vorticity contours and density lines for shock-induced collapse for different resolutions . . . . . . . . . . . . . . . . 78

3.18 Circulation history for different resolutions . . . . . . . . . . . . 78

3.19 Bubble volume history for different resolutions . . . . . . . . . . . 79

3.20 Density lines and numerical Schlieren contours for different resolutions 80

4.1 Solution to the Rayleigh-Plesset equation for Rayleigh collapse . . 86

4.2 Acceleration of the bubble interface for Rayleigh collapse . . . . . 86

4.3 Phase diagram for a bubble cycle for Rayleigh collapse . . . . . . . 87

4.4 Pressure in the liquid as a function of distance for different times for Rayleigh collapse . . . . . . . . . . . . . . . . . . . . . 89

4.5 Pressure in the liquid as a function of distance for different times to highlight the bifurcation. . . . . . . . . . . . . . . . . 90

4.6 Pressure and numerical Schlieren contours for Rayleigh collapse in a free field $\left(p_{s} / p_{o}=34\right) \ldots \ldots \ldots \ldots . \ldots . \ldots 95$

4.7 Pressure and numerical Schlieren contours of shock-induced collapse in a free field $\left(p_{s} / p_{o}=353\right) \ldots \ldots \ldots \ldots 7$

4.8 Pressure and numerical Schlieren contours for Rayleigh collapse near a wall $\left(p_{s} / p_{o}=34, H_{o} / R_{o}=1.5\right) \ldots \ldots \ldots . \ldots 98$ 
4.9 Pressure and numerical Schlieren contours for shock-induced collapse near a wall $\left(p_{s} / p_{o}=353, H_{o} / R_{o}=1.5\right) \ldots \ldots \ldots$

4.10 Chronology of events in free-field shock-induced collapse . . . . . . 102

4.11 Chronology of events as a function of the pressure ratio across the shock for free-field shock-induced collapse. . . . . . . . . . . . . . . 102

4.12 Initial velocity of the interface for Rayleigh collapse and shockinduced collapse . . . . . . . . . . . . . . 105

4.13 Pressure ratio and Mach number of the transmitted shock for Rayleigh collapse and shock-induced collapse . . . . . . . . . . . . . 106

4.14 Numerical Schlieren contours for Rayleigh collapse for different resolutions . . . . . . . . . . . . . . . . 107

4.15 History of the bubble non-sphericity . . . . . . . . . . . . 109

4.16 Maximum bubble non-sphericity . . . . . . . . . . . . . . . . . 110

4.17 Maximum velocity of the jet and distal side for free-field Rayleigh collapse and shock-induced collapse . . . . . . . . . . . . . . 111

4.18 Maximum velocity of the jet and distal side as a function of the initial stand-off distance for Rayleigh collapse near a wall . . . . . 112

4.19 Maximum velocity of the jet and distal side as a function of the initial stand-off distance for shock-induced collapse near a wall . . 113

4.20 Jet outline for free-field shock-induced collapse . . . . . . . . . . . 114

4.21 Jet size and bubble height as a function of the pressure ratio for free-field shock-induced collapse . . . . . . . . . . . . . . . 115

4.22 Collapse time as a function of the initial pressure ratio for free-field Rayleigh collapse and shock-induced collapse . . . . . . . . . . 116

4.23 Collapse time as a function of the initial stand-off distance for Rayleigh collapse and shock-induced collapse near a wall . . . . . 117

4.24 History of the bubble displacement for Rayleigh collapse near a wall 119

4.25 Distance of the bubble from the wall at collapse for Rayleigh collapse and shock-induced collapse . . . . . . . . . . . . . . . 120 
xviii

4.26 Total circulation and rate of change of circulation for free-field shock-induced collapse . . . . . . . . . . . . . . . . . 122

4.27 Total circulation and rate of change of circulation for Rayleigh collapse near a wall . . . . . . . . . . . . . . . . . . 123

4.28 Circulation after the propagation of the internal shock through the entire bubble in free-field shock-induced collapse . . . . . . . . . .

4.29 Collapse of a bubble based on the flow induced by the passage of the shock . . . . . . . . . . . . . . 127

4.30 Non-spherical perturbations of the bubble interface . . . . . . . . . 129

4.31 Jet velocity and bubble non-sphericity in jet formation for shockinduced collapse . . . . . . . . . . . . . . . 133

4.32 Jet velocity as a function of the interface position for free-field shockinduced collapse . . . . . . . . . . . . . . . . . . . . . 134

4.33 Jet velocity and bubble non-sphericity in jet formation for Rayleigh collapse near a wall . . . . . . . . . . . . . . . . 136

4.34 Initial flow field for free-field Rayleigh collapse . . . . . . . . . . . 139

4.35 Flow field for free-field Rayleigh collapse just before and after collapse140

4.36 Flow field just before the impact of the jet onto the distal side for Rayleigh collapse near a wall . . . . . . . . . . . . . . . . . . 142

4.37 Flow field just after the impact of the jet onto the distal side for Rayleigh collapse near a wall . . . . . . . . . . . . . . . . . 143

4.38 Flow field as the shock diffracts off the bubble for free-field shockinduced collapse . . . . . . . . . . . . . . . . . . . . . . 144

4.39 Flow field just before the jet hits the distal side for free-field shockinduced collapse ...................... 145

4.40 Flow field when the water-hammer is generated for free-field shockinduced collapse . . . . . . . . . . . . . . . . . . 147

4.41 Flow field just after the jet hits the distal side for free-field shockinduced collapse . . . . . . . . . . . . . . . . . . . . 148

4.42 Water-hammer pressure . . . . . . . . . . . . . . 149 
4.43 Fraction of radiated energy and maximum bubble pressure for freefield shock-induced collapse . . . . . . . . . . . . . . . . . . 151

4.44 Fraction of radiated energy and maximum bubble pressure for Rayleigh collapse near a wall . . . . . . . . . . . . . . . . . 152

4.45 History of the wall pressure for shock-induced collapse near a wall 153

4.46 Maximum wall pressure for shock-induced collapse . . . . . . . . . 155

4.47 Maximum wall pressure for shock-induced collapse with comparisons to experiments . . . . . . . . . . . . 156

4.48 Wall pressure along the centerline for shock-induced collapse . . . 157

4.49 Maximum wall pressure for Rayleigh collapse . . . . . . . . . . . . 158

4.50 Averaged wall pressure for Rayleigh collapse . . . . . . . . . . . 159

4.51 Maximum wall pressure for shock-induced collapse near a wall . . . 161

4.52 Maximum wall pressure due to the shock for shock-induced collapse near a wall . . . . . . . . . . . . . . . . . 162

5.1 Pressure waveform of a lithotripter pulse at the focus . . . . . . . 167

5.2 Planar shock reflection off a solid surface . . . . . . . . . . . . . 168

5.3 Pressure and numerical Schlieren contours for shock-induced collapse in SWL . . . . . . . . . . . . . . . . . . . 169

5.4 History of the bubble volume and displacement and velocity of the jet and of the distal side for shock-induced collapse in SWL . . . . 170

5.5 History of the wall pressure and pressure profiles along the wall for shock-induced collapse in SWL . . . . . . . . . . . . . . . . . 172

5.6 Wall pressure along the centerline as a function of the initial standoff distance for two different bubble sizes . . . . . . . . . . . . . . . 174

5.7 Collapse time and bubble displacement as a function of the pulse width for free-field shock-induced collapse in SWL . . . . . . . . . 175

5.8 Maximum velocity of the jet and distal side and water-hammer pressure as a function of the pulse width for free-field shock-induced collapse in SWL . . . . . . . . . . . . . . . 176 
5.9 Bubble pressure and normalized radiated energy at collapse for shock-induced collapse in SWL . . . . . . . . . . . . . . 177

5.10 Circulation of the transmitted shock and maximum circulation for shock-induced collapse in SWL . . . . . . . . . . . . . 177

5.11 Wall pressure along the centerline as a function of pulse width for different initial stand-off distances in SWL . . . . . . . . . . . . . 178

5.12 Waveform for increasing pulse widths . . . . . . . . . . . 179

5.13 Maximum tensile stress for a $2 \mathrm{~mm}$-radius stone over the course of the elastic waves simulation . . . . . . . . . . . . . . . 184

5.14 Maximum tensile stress for a $1 \mathrm{~mm}$-radius stone over the course of the elastic waves simulation . . . . . . . . . . . 185

A.1 Schematic of the reference frames for the shock . . . . . . . . 198

A.2 Shock speed vs. particle speed in water . . . . . . . . . 200

A.3 Space-time diagram for expansion waves . . . . . . . . . . . 202 


\section{List of Tables}

2.1 Fluid properties . . . . . . . . . . . . . . . . . . 31

2.2 Definition of the different problems under consideration . . . . . 33

3.1 Definitions of variables used in the derivation of the WENO procedure 41

3.2 Computation of characteristic variables at the boundaries . . . . 55

3.3 Comparison between the different FD and FV WENO schemes . . 57

4.1 Events of interest during bubble collapse . . . . . . . . . . . . . 101

4.2 Perturbation velocity due to baroclinic vorticity for shock-induced collapse ............................ 131

5.1 Representative values of the pulse width and initial bubble radius for an electro-hydraulic lithotripter . . . . . . . . . . . . 175

A.1 Values of $\gamma, P_{\infty}$ used in the literature, with the corresponding shock Mach number . . . . . . . . . . . . . . . . . . 201 
xxiii

\section{List of Publications}

\section{Journal articles}

E. Johnsen, R. O. Cleveland, and T. Colonius, "Internal structural damage caused by bubble collapse on neighboring solid surfaces", in preparation (2008).

E. Johnsen and T. Colonius, "Numerical simulations of non-spherical bubble collapse", J. Fluid Mech., in preparation (2008).

E. Johnsen and T. Colonius, "Shock-induced collapse of a gas bubble in shockwave lithotripsy", J. Acoust. Soc. Am., submitted (2008).

E. Johnsen and T. Colonius, "Implementation of WENO schemes in compressible multicomponent flow problems", J. Comput. Phys. 219 (2006) 715-732.

\section{Conference proceedings}

E. Johnsen, T. Colonius, W. Kreider, and M. R. Bailey, Axisymmetric collapse of an air bubble subjected to a lithotripter pulse, International Mechanical Engineering Congress and Exposition, American Society of Mechanical Engineers, Seattle, USA (2007).

E. Johnsen and T. Colonius, Numerical simulation of the collapse of a bubble subjected to a lithotripter pulse, 1st Annual International Urolithiasis Research Symposium, International Kidney Stone Institute, Indianapolis, USA (2006).

E. Johnsen and T. Colonius, Computations of shock-bubble interaction and aspherical bubble collapse, Sixth International Symposium on Cavitation, Maritime Research Institute, Wageningen, Netherlands (2006). 
xxiv 


\section{Nomenclature}

\section{Roman characters}

A Atwood number

c Sound speed

d Ideal WENO weight

e $\quad$ Specific internal energy

f Flux vector

E Total energy per unit volume

E Total energy

$h \quad$ Specific enthalpy

$H \quad$ Distance of the bubble from the wall

$l \quad$ Characteristic jet size

$M \quad$ Mach number

M Total mass

$M U$ Total momentum

$N \quad$ Number of points

$p \quad$ Pressure

$p_{o} \quad$ Ambient pressure

$p_{s} \quad$ Pressure behind the shock

$p_{v} \quad$ Vapor pressure

$P_{\infty} \quad$ Stiffness constant 
q Vector of conservative variables

$R$ Bubble radius

$r$ Radial coordinate

$s \quad$ Shock speed

$s \quad$ Specific entropy

$s \quad$ Wave speed in HLL solvers

$S$ Surface tension

$t$ Time

$T$ Temperature

$u \quad$ Velocity

$u$ Velocity component in the axial direction $(x)$

u Velocity vector

u Vector of characteristic variables

$v \quad$ Velocity

$v \quad$ Velocity component in the radial direction $(r)$

$v \quad$ Specific volume

V Volume

$x \quad$ Axial coordinate

\section{Greek characters}

$\beta \quad$ WENO smoothness indicators

$\Delta r \quad$ Grid spacing in the $r$-direction

$\Delta V \quad$ Volume element

$\Delta x \quad$ Grid spacing in the $x$-direction

$\gamma \quad$ Ratio of specific heats (gases)

$\Gamma \quad$ Circulation

$\Gamma \quad$ Fundamental gasdynamic derivative 
$\lambda \quad$ Eigenvalues of the Euler equations

$\lambda$ Dilatation

$\Lambda \quad$ Eigenvalue matrix

$\mu \quad$ Dynamic viscosity

$\nu \quad$ Kinematic viscosity

$\nu \quad$ Bubble non-sphericity

$\rho$ Density

$\sigma \quad$ Width of the lithotripter pulse

$\xi \quad$ Volume fraction

$\phi \quad$ Vector of advected variables

$\omega$ WENO weight

$\boldsymbol{\omega}$ Vorticity

\section{Subscripts}

$Q_{\infty} \quad$ Value at infinity

$Q_{B} \quad$ Property of the bubble

$Q_{C D} \quad$ Property of the contact discontinuity

$Q_{d} \quad$ Value of $Q$ at the distal side

$Q_{\text {dist }}$ Value of $Q$ at the distal side

$Q_{G} \quad$ Property of the gas

$Q_{i j} \quad$ Value of $Q$ at coordinates $\left(x_{i}, r_{j}\right)$

$Q_{j} \quad$ Value of $Q$ at the jet

$Q_{\text {jet }} \quad$ Value of $Q$ at the jet

$Q_{L} \quad$ Property of the liquid

$Q_{o} \quad$ Initial value of $Q$

$Q_{s} \quad$ Property behind the shock

$Q_{\text {wall }}$ Property at the wall 


\section{Superscripts}

$\bar{Q} \quad$ Cell-average value of $Q$

$\dot{Q} \quad$ Derivative of $Q$ with respect to time

$Q^{L} \quad$ Value of $Q$ at the left of a cell edge

$Q^{(n)} \quad$ Value of $Q$ at time level, $n$

$Q^{(r)} \quad r$ th-order WENO polynomial

$Q^{R} \quad$ Value of $Q$ at the right of a cell edge

\section{Abbreviations}

ENO Essentially non-oscillatory

FD Finite difference

FV Finite volume

HLL Harten-Lax-van Leer solver

HLLC HLL solver with built-in contact discontinuity

$\mathrm{RC} \quad$ Rayleigh collapse

RCW Rayleigh collapse near a wall

RHS Right-hand side

RK Runge-Kutta

SIC Shock-induced collapse

SICW Shock-induced collapse near a wall

TVD Total variation diminishing

WENO Weighted ENO 


\section{Chapter 1}

\section{Bubble collapse and cavitation damage}

The present chapter provides an introduction to cavitation and the structural damage caused by bubble collapse. First, the concept of cavitation is explained. Then, the phenomenon of damage due to bubble collapse is examined, including examples such as shockwave lithotripsy, which constitutes the motivation for the current work. Next, the collapse of a single bubble is presented through a historical overview of the problem, from analytical, experimental and numerical standpoints. This chapter ends with a list of the contributions of the present work to the field of numerical methods in bubble dynamics and an overview of the thesis.

\subsection{The occurrence of bubbles in flows of liquids}

When the local tension in a liquid (i.e., the difference between the saturated vapor pressure and the local pressure) is smaller than the tensile strength, the liquid may rupture. When the resulting microscopic cavity grows to a macroscopic bubble due to vaporization of the liquid, cavitation is said to occur (Knapp et al., 1970; Brennen, 1995). ${ }^{1}$ This phenomenon usually takes place at specific locations in the liquid where imperfections, such as dissolved gas or small particles, are present. When exposed to a transient tension in the liquid, cavities expand at these nucleation sites and grow to a maximum radius ranging from hundreds of microns to

\footnotetext{
${ }^{1}$ This phenomenon should be contrasted with boiling, in which the change in temperature is the mechanism driving the rupture of the liquid.
} 
centimeters in most engineering applications (Knapp et al., 1970). ${ }^{2}$ The bubbles then collapse violently to a small size prescribed by the amount of non-condensible gas contained within (Plesset \& Prosperetti, 1977), sometimes dissolving into the liquid after a single cycle.

Reductions in pressure leading to cavitation are typically caused by two factors (Li, 2000): local variations due to the hydrodynamics of the flow field (i.e., the Bernouilli effect) or the application of an acoustic field. Accordingly, the former is called hydrodynamic cavitation and is characterized by the fact that a given liquid element usually only passes through a cavitation zone only once. This phenomenon frequently occurs in naval structures and turbomachines, as described in the first half of Section 1.2.3. The latter is termed acoustic cavitation and is usually characterized by small velocity fluctuations; acoustic waves lead to regions of reduced pressure where cavitation takes place. This process is exploited in several bio-medical applications, as discussed in the second half of Section 1.2.3.

Because of the dynamical interaction of bubbles with the flow field, the occurrence of cavitation in hydraulic systems can lead to a number of drawbacks, such as a decline in performance and efficiency, or an increase in noise and vibrations (Franc \& Michel, 2004). One of the most severe consequences of cavitation is the high pressure generated when bubbles collapse and the structural damage resulting when this process occurs near a solid surface.

\subsection{Cavitation damage}

\subsubsection{The violence of bubble collapse}

Although the collapse of a bubble in a liquid may seem like an innocuous event, extreme physics may be observed during the process. From its maximum radius to

\footnotetext{
${ }^{2}$ In a number of applications, vapor cavities of a larger scale (e.g., up to meters) form due to a high density of cavitation events (Knapp et al., 1970; Arndt, 1981). Examples include vortex cavitation, where regions of high vorticity lead to low pressures, and attached cavitation, where a region of separated flow is filled with vapor. However, these flow phenomena are beyond the scope of the present study.
} 
collapse, the volume of a cavitation bubble decreases rapidly by several orders of magnitude. As this nearly adiabatic compression occurs, the pressure and temperature within the cavity reach high values, and shockwaves are emitted (Hickling \& Plesset, 1964). In fact, Suslick (1990) remarks that by using conventional ultrasound equipment to drive a bubble to oscillate near its natural frequency,

[...] one can create the temperature of the sun's surface, the pressure of deep oceanic trenches, and the cooling rate of molten metal splatted onto a liquid-helium-cooled surface!

Such conditions can give rise to a number of interesting phenomena, including sonoluminescence (Putterman \& Weninger, 2000), where light is emitted upon collapse, sonochemistry (Suslick, 1990), where chemical reactions rates are substantially improved, or sonofusion (Taleyarkhan et al., 2002), where experimenters claimed to have observed nuclear emissions. ${ }^{3}$ In sonoluminescence, temperatures up to $15,000 \mathrm{~K}$ (McNamara III et al., 1999) and pressures greater than $0.37 \mathrm{GPa}$ (Flannigan et al., 2006) have been measured in experiments, thus demonstrating the tremendous destructive potential of cavitation bubbles.

\subsubsection{Undesirable cavitation damage}

Conditions such as those described in the preceding section are uncommon in most engineering applications, where cavitation is usually of a hydrodynamic nature. Nevertheless, Suslick's comment illustrates the tremendous damage potential of even a single bubble collapsing near a structure. Half a century ago, Robert Knapp (1952) stated in his James Clayton Lecture,

Cavitation is one hydraulic phenomenon in which the effects are predominantly undesirable and often destructive.

\footnotetext{
${ }^{3}$ The experiments of Taleyarkhan et al. (2002) where fusion evidence was observed could not be reproduced by other researchers and constitutes an on-going controversy in the community (Riesch, 2007).
} 
At the time, the focus of cavitation research lay in the need to understand to what extent bubbles affected the operation of naval structures. In an investigation of the reasons for the sub-par operation of a British destroyer, Thornycroft \& Barnaby (1895) were the first to report on the reduced performance and structural damage caused by cavitation. ${ }^{4}$ Historically, naval engineering has been and still is one of the main areas of cavitation research as collapsing bubbles and the resulting damage may be observed along propellers, rudders, struts, and even hulls, (Knapp et al., 1970).

Turbomachines constitute another field where cavitation plays a deleterious role. In certain flows of liquids through rotating machines such as pumps and turbines, cavitation cannot be avoided ( $\mathrm{Li}, 2000)$. The operation of valves and nozzles may also be affected by cavitation, due to changes in the velocity of the liquid passing through them (Knapp et al., 1970). Thus, care must be taken in the design of such instruments in order to minimize the destructive action of cavitation (Brennen, 1994).

Damage caused by bubble collapse is observed over a wide range of scales and in different types of liquids. One of the most spectacular examples is the cavitation erosion sustained by the passage of a large flood through a spillway or the outlet of a dam, as illustrated by the size of the eroded parts compared to height of the person in Figure 1.1 (Arndt, 1981). Furthermore, cavitation damage is not restricted to flows of water. In the Spallation Neutron Source at Oak Ridge National Laboratory, short-pulse proton beams strike heavy-metal targets containing liquid mercury; because of the intense heating, large pressure variations may lead to the formation of cavitation bubbles, which then collapse and generate significant damage (Haines et al., 2005). On a smaller scale, examples of cavitation damage in the bio-medical field include damage to red blood cells in artifical heart valves (Yoganathan et al., 2004) or trauma due to impact head injuries (Lubock \& Goldsmith, 1980).

\footnotetext{
${ }^{4}$ It was in the context of the research of Thornycroft \& Barnaby (1895) that R. E. Froude coined the term cavitation.
} 


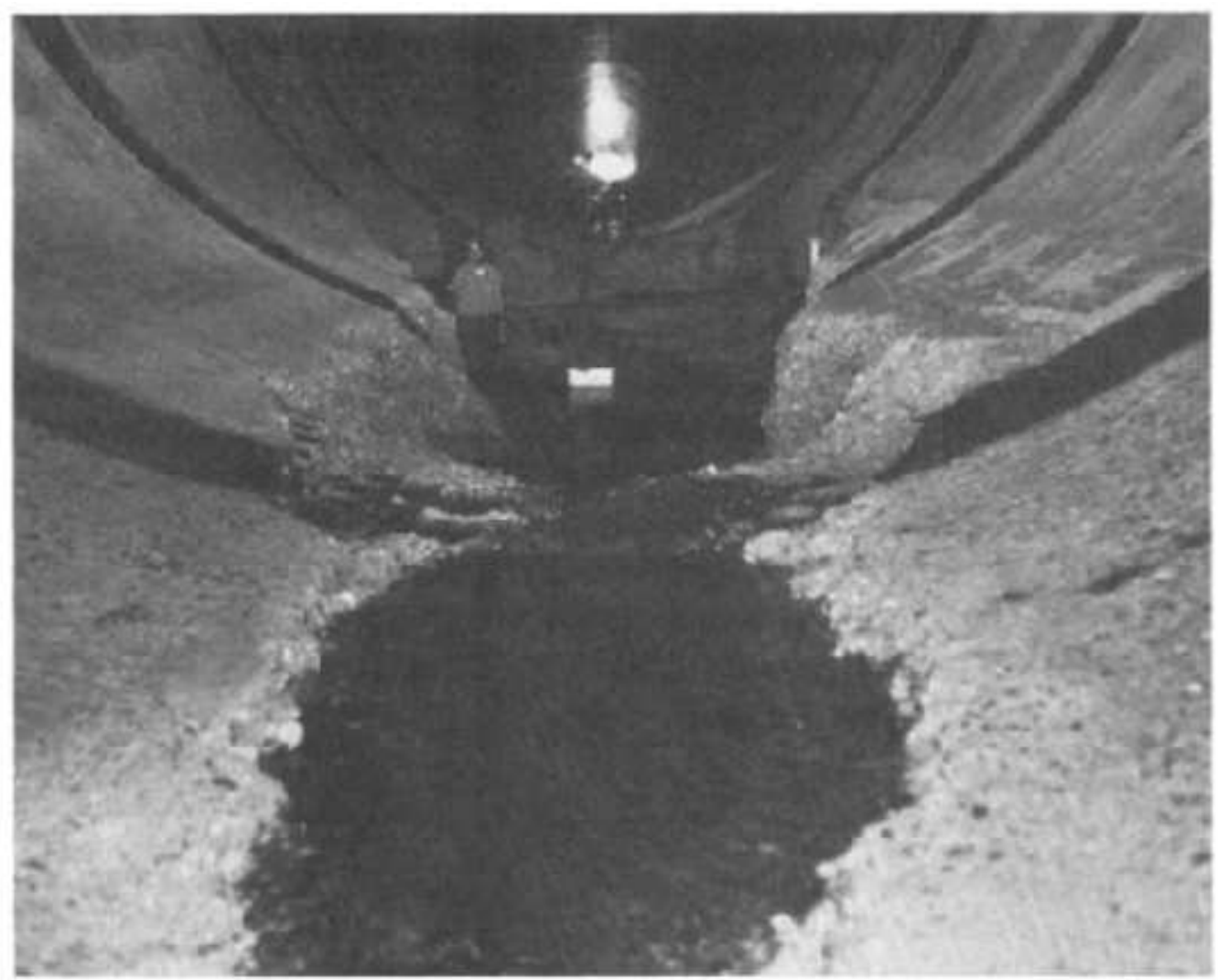

Figure 1.1: Cavitation damage in the spillway tunnel of the Yellowtail Dam, Montana (Arndt, 1981). Courtesy of the US Bureau of Reclamations.

In underwater explosions, the effects of cavitation are important. A large amount of energy is released from a small source, which generates a strong shockwave propagating radially outwards, with substantial damage potential (Cole, 1948). A gas bubble containing the products of the explosion rapidly expands to a large size (on the order of meters) and subsequently collapses violently. The dynamics of the gas bubble are complex, due to buoyancy effects and the possible presence of nearby free-surfaces or solid structures, and large quantities of water may be displaced during the process (Holt, 1977). However, cavitation damage seems to occur on a small scale. As described in Figure 1.2, the shockwave resulting from the underwater explosion loads the target, prompting it to recoil and create a region of low pressure (Wardlaw \& Luton, 2000). After reflecting off the target, 
the shock interacts with the explosion bubble and is reflected as an expansion wave due to the impedance mismatch. This leads to a further pressure decrease near the target, providing conditions favorable to the creation of cavitation bubbles, which may inflict significant damage to the structure upon collapse.

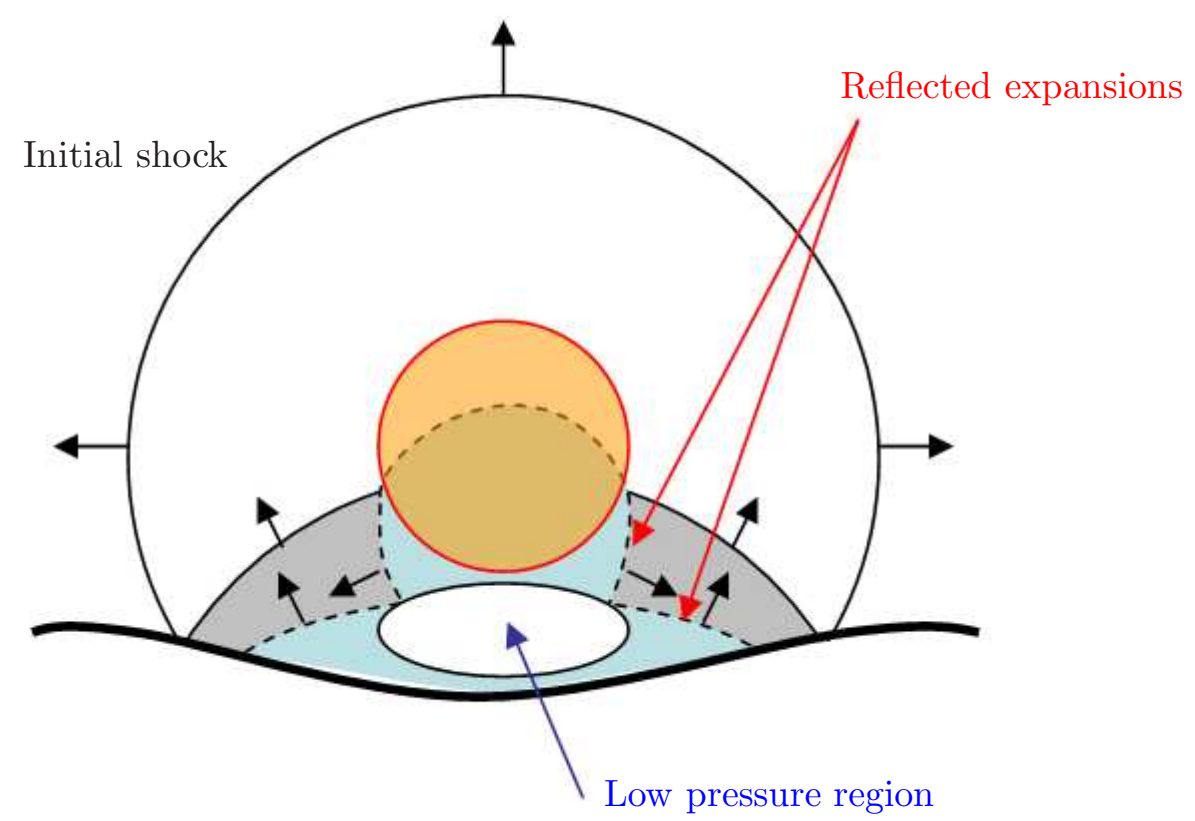

Figure 1.2: Schematic of an underwater explosion, adapted from Wardlaw \& Luton (2000).

\subsubsection{Desirable cavitation damage}

The examples mentioned in the previous section constitute a non-exhaustive list of drawbacks of cavitation damage. However, in a number of applications, cavitation erosion is employed in a deliberate fashion to clean or break materials. In these instances, cavitation is usually controlled by acoustic means.

In ultrasonic cleaning, the flow induced by a bubble oscillating stably under the action of an acoustic field (i.e., microstreaming (Elder, 1959)) generates shear stresses that remove particulates from surfaces. Wet finishing, where textile materials are treated in an attempt to modify their properties (Moholkar et al., 2004), 
and silicon wafer cleaning (Busnaina et al., 1995) are examples of this process. Ohl et al. (2006) recently suggested that the main contributions to the shear flow in ultrasonic cleaning emanate from the spreading of the liquid jet generated during bubble collapse along surfaces.

The use of cavitation bubble collapse to an advantage has even been observed in animals. When the snapping shrimp closes its claw, it generates a high-speed jet, which in turn leads to a local pressure below vapor pressure (Versluis et al., 2000). Cavitation bubbles are thereby generated and their collapse provides means for snapping shrimps to communicate amongst each other and stun their prey.

Several bio-medical applications use cavitation in a deliberate and controlled fashion. In dentistry, plaque is removed using an ultrasonically vibrating probe; Walmsley et al. (1988) showed that cavitation occurring in the cooling water supply as it passes over the tip contributes to the plaque removal process. Such research has translated into the mainstream sonic toothbrush and, more recently, into its ultrasonic sibling (Mourad et al., 2007). In cataract surgery, the eye's natural lens is emulsified and removed in a process called phacoemulsification (Packer et al., 2005). Bubbles also play an important role in shockwave lithotripsy, which is described in greater detail below; the necessity to better understand certain aspects of this medical procedure, in particular the role of bubble collapse in the stone comminution process, constitutes the main motivation for the present work.

\section{Shockwave lithotripsy}

Over the course of their life, 5-12\% of Americans will develop a kidney stone (Coe et al., 2005). Most stones are made of calcium oxalate and calcium phosphate; they usually form because these substances dissolved in urine within the urinary tract solidify. If small enough, the stones can be passed naturally by the body; however, when they are large ${ }^{5}$ or located in certain strategic sites, they can lead to severe pain and must therefore be removed.

\footnotetext{
${ }^{5}$ Kidney stones can reach a size of several centimeters.
} 
The most common form of treatment is a non-invasive medical procedure called (extracorporeal) shockwave lithotripsy (SWL) (NIH, 2004), which has been used since the 1980s (Chaussy, 1982). The patient is typically immersed in a water bath, while shockwaves generated underwater are focused onto the kidney stone in an attempt to break them. Current devices include electrohydraulic lithotripters, in which the shock is generated using electrodes and is focused using a reflector, electromagnetic lithotripters, in which acoustic pulses are focused using a paraboloid reflector or a lens, and piezoelectric lithotripters, which consist of an array of spherically aligned piezoelectric elements.

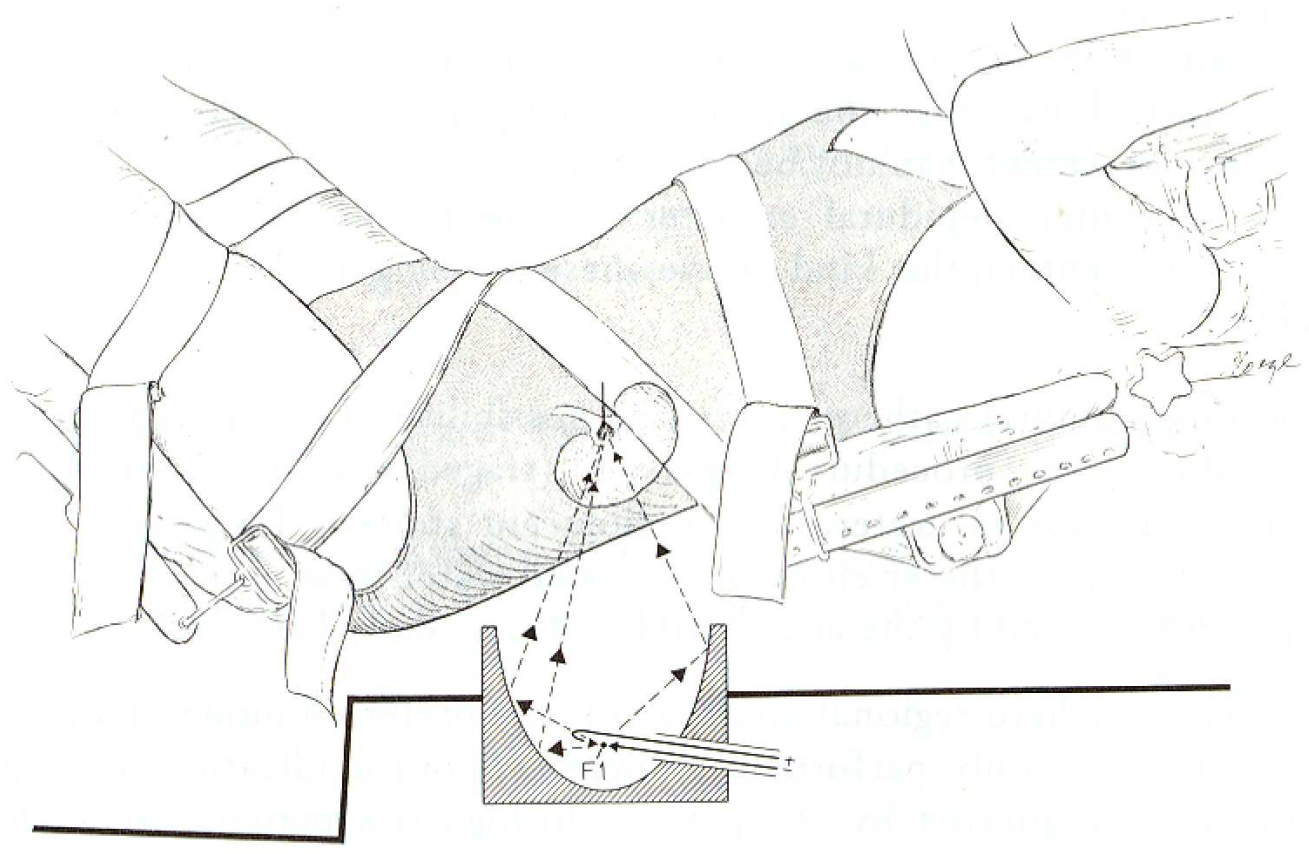

Figure 1.3: Schematic of the set-up for SWL using an electrohydraulic lithotripter. Reproduced with permission from Chaussy (1986). Copyright 1986 by Karger AG, Basel.

A typical electrohydraulic lithotripter consists of a reflector in the shape of a truncated ellipsoid, with a sparkplug in one focus. The patient is immersed in a water bath, such that the kidney stone is located at the second focus, as shown in Figure 1.3 (Chaussy, 1986). When the spark is generated, a shockwave propagates 
radially outwards, and, upon reflection, is focused onto the kidney stone. In a typical procedure, several hundreds to thousands of shockwaves are fired at a rate of approximately $0.5 \mathrm{~Hz}$ (Paterson et al., 2002; Willis et al., 2005). As a result, the stones are broken into fragments small enough that they can be passed naturally by the human body. The main reason for the popularity of SWL is the fact that surgery is avoided altogether. However, collateral damage to the kidney may be caused during the process, on the cellular (Lokhandwalla \& Sturtevant, 2001; Sapozhnikov et al., 2002) and systemic (Evan et al., 1998, 2002) levels. In addition to kidney stones, shockwave lithotripsy has been used to treat gall stones, pancreatic stones, salivary stones and bone fracture (Delius, 1994).

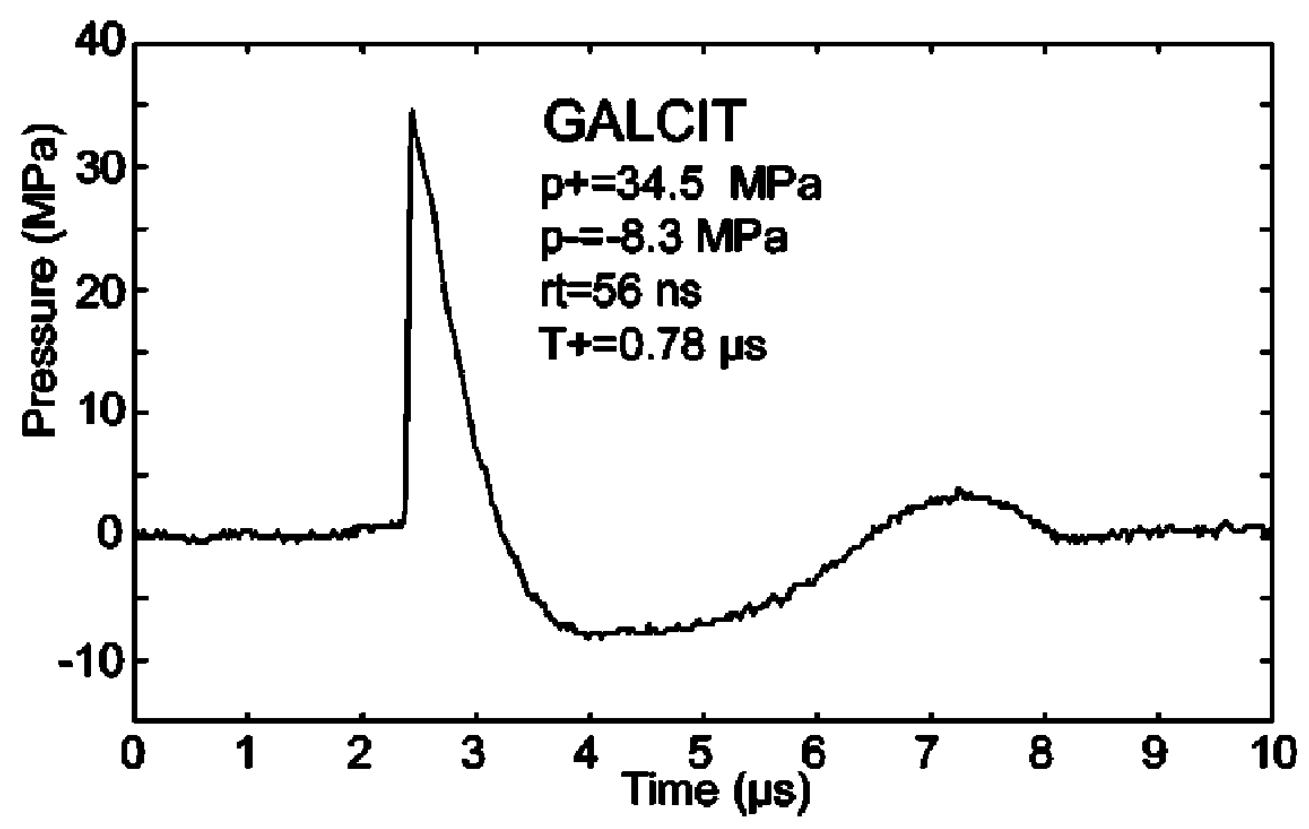

Figure 1.4: Pressure profile in the focal region of a lithotripter (Cleveland et al., 2000).

Because of the complex physics governing the process, the precise stone comminution mechanisms are not yet fully understood (Eisenmenger, 2001). As shown in Figure 1.4 (Cleveland et al., 2000), the lithotripter pulse in the focal region consists of a steep compressive front followed by a long expansion tail with a tensile 
component. Since kidney stones are typically immersed in urine and possibly in pooled blood, this tension leads to the formation of bubbles at nuclei sites near the stone. Two mechanisms are thus important in stone comminution: shockwave propagation within and around the stone (Lokhandwalla \& Sturtevant, 2000), which may lead to spallation (Gracewski et al., 1993), squeezing (Eisenmenger, 2001) or the constructive interference of shear waves (Cleveland \& Sapozhnikov, 2005), and cavitation erosion due to violent bubble collapse along the surface (Coleman et al., 1987; Crum, 1988). Artifical stones damaged by spallation and cavitation are shown in Figure 1.5.
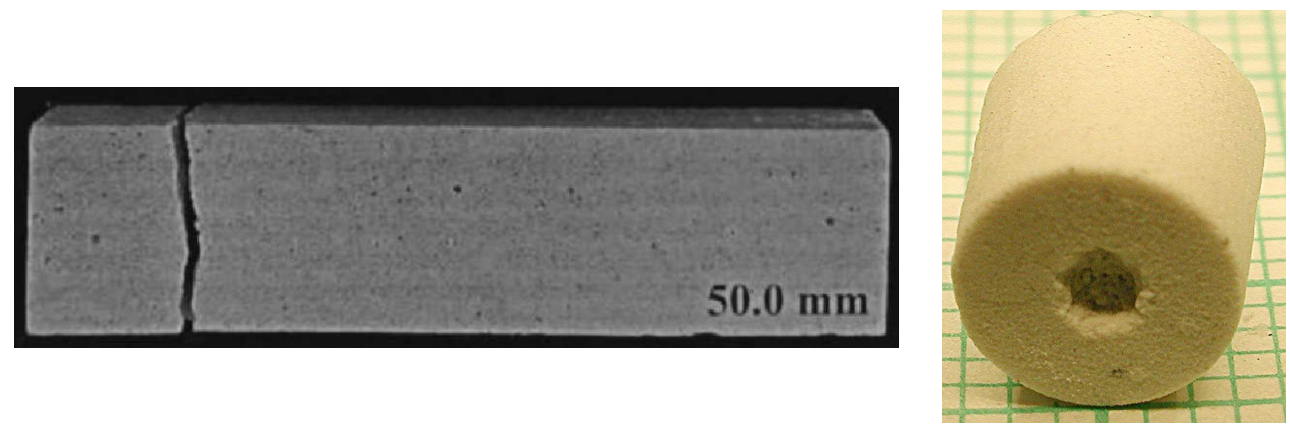

Figure 1.5: Damage on artificial kidney stones. Left: spallation (adapted with permission from Xi \& Zhong (2001), copyright 2001 by the Acoustical Society of America; right: cavitation erosion (Pishchalnikov et al., 2003).

Experimental findings suggest that the combined effect of the internal stress waves and cavitation erosion pulverizes the stones (Zhu et al., 2002); if either cavitation or the wave propagation within the stone are inhibited, the comminution efficiency decreases. Though repeated bubble collapse may lead to significant structural damage, the individual impact of bubble dynamics on stone comminution is still unclear. After the passage of a lithotripter pulse, bubbles generally gather in clusters that grow and collapse near the stone surface, thus generating shockwaves (Xi \& Zhong, 2001; Pishchalnikov et al., 2003). The effect of bubble clouds in SWL has been studied numerically by Tanguay (2004), who showed that there exists an optimal bubble population for which the damage potential is maximized, while stone shielding is minimized. It is clear (at least in vitro) that 
nucleation of bubbles takes place at existing gas nuclei, which become more numerous as the treatment progresses (Pishchalnikov et al., 2003). Thus, in addition to cavitation bubble collapse (Rayleigh, 1917), which occurs in SWL at a time $\mathrm{O}(100)$ microseconds after the initial tensile portion of the shockwave, pre-existing gas bubbles are collapsed on a scale of microseconds by the compressive portion of the pulse (i.e., the shock).

\subsection{Single-bubble dynamics}

Whether cavitation damage is desirable or not, an understanding of the basic physics leading to this phenomenon is necessary. A number of attempts to classify the various types of cavitation have been made in the literature (Knapp et al., 1970; Brennen, 1995; Franc \& Michel, 2004). Acosta (2000) even tabulated twelve different regimes likely to occur in pumps or turbines! The most common type of cavitation in SWL is a combination between cloud cavitation, where a collection of bubbles behaves as a coherent whole, and acoustic cavitation, which is described in Section 1.1. Historically, before considering the complex dynamics of a cloud, the fundamental problem of the transient growth and collapse of a single bubble subjected to time-varying pressure fields was first examined. ${ }^{6}$

\subsubsection{Theoretical and experimental studies}

Starting with the research of Lord Rayleigh (1917), single-bubble collapse has been studied for almost a century. Besant (1859) had solved the following theoretical problem previously:

An infinite mass of homogeneous incompressible fluid acted upon by no forces is at rest, and a spherical portion of the fluid is suddenly annihilated; it is required to find the instantaneous alteration of pressure at any point of the mass, and the time in which the cavity will be

\footnotetext{
${ }^{6}$ In collections of bubbles, if the number density is low enough, a given bubble may be assumed to behave independently from its neighbors (Knapp et al., 1970).
} 
filled up, the pressure at an infinite distance being supposed to remain constant.

However, Rayleigh was the first to fully appreciate the engineering implications of bubbles repeatedly collapsing along propeller blades, and his studies set the basis for cavitation research henceforth (Hammitt, 1980). Because the time scale of bubble collapse is typically much smaller than the heat diffusion and conduction time scales, the compression of the bubble during collapse can be assumed adiabatic; this implies that a large amount of energy is concentrated into a tiny volume. The high pressures achieved during collapse and the impact of shockwaves thereby emitted were the first proposed cavitation damage mechanisms (Rayleigh, 1917; Hickling \& Plesset, 1964; Fujikawa \& Akamatsu, 1980). Many improvements to Rayleigh's theory of spherical bubble dynamics have been made over the last century. In studies of traveling cavitation bubbles, Plesset (1949) included viscous and surface tension effects into what is now known as the Rayleigh-Plesset equation. Gilmore (1952) further extended the theory to include liquid compressibility effects. Thermal effects were considered by Plesset \& Zwick (1954) and Hickling (1963). Further extensions and studies can be found in Plesset \& Prosperetti (1977) and Brennen (1995).

In the middle of the twentieth century, it was appreciated that the asymmetry in the flow field caused by the presence of a solid surface near a bubble would lead a re-entrant jet to form during the collapse. Kornfeld \& Suvorov (1944) first proposed this flow phenomenon, which was later confirmed by the experiments of Naudé \& Ellis (1961) and Benjamin \& Ellis (1966), who also considered the problem analytically. During the collapse, the far end of the bubble involutes, such that a high-speed re-entrant jet directed towards the solid surface forms and penetrates the bubble during collapse, as shown in the recent high-speed photographs of Lindau \& Lauterborn (2003) in Figure 1.6. If such a bubble is close enough to the wall, the jet may directly impact it and generate a large water-hammer pressure (Plesset \& Chapman, 1971). 
Most early experiments employed a spark discharge method to vaporize a small portion of liquid and generate a bubble (Naudé \& Ellis, 1961; Benjamin \& Ellis, 1966; Kling \& Hammitt, 1972). Using this technique, Tomita \& Shima (1986) studied the collapse of isolated cavitation bubbles near a solid surface and measured wall pressures up to $12 \mathrm{MPa}$. They found that bubbles near the wall migrate towards it significantly and further argued that cavitation damage was caused by the collapse of tiny bubbles generated during the collapse of the main bubble. However, electrodes interfer with the flow field, and the bubbles thereby generated are not always spherical. In order to study spherical bubble collapse in a controlled and repeatable fashion, optical cavitation was developed, where a laser is used to vaporize water, thus creating a bubble (Lauterborn \& Bolle, 1975). The combination of this technique with high-speed photography has provided many advances in understanding the phenomenon of bubble collapse near a wall and provided important insight into cavitation erosion. Vogel et al. (1989) investigated the formation of the jet and the effect of the wall on the bubble dynamics. Philipp \& Lauterborn (1998) determined that bubbles in direct contact with solid surface were responsible for the largest part of the erosion. Lindau \& Lauterborn (2003) visualized shockwaves emitted during collapse and studied the properties of the counterjet formed after the collapse. However, the method by which such cavitation bubbles are generated may lead to dynamical features different from those observed in engineering applications (Lauterborn \& Bolle, 1975).

When the flow is inviscid, the collapse of a bubble near a rigid wall is equivalent to the simultaneous collapse of two identical bubbles. Another type of asymmetry consists of the presence of an external force field, such as gravity. In such problems, a re-entrant jet forms in the direction of the buoyancy force (Walters \& Davidson, 1962). The passage of a shockwave over a bubble also leads to the formation of a re-entrant jet in the direction of propagation of the shock (Tomita \& Shima, 1986). In cloud cavitation, shockwaves may be generated by the collapse of individual bubbles, as explained previously, or by the coherent collapse of the cloud (Hansson et al., 1982). In SWL, the incoming pulse may interact with pre-existing 


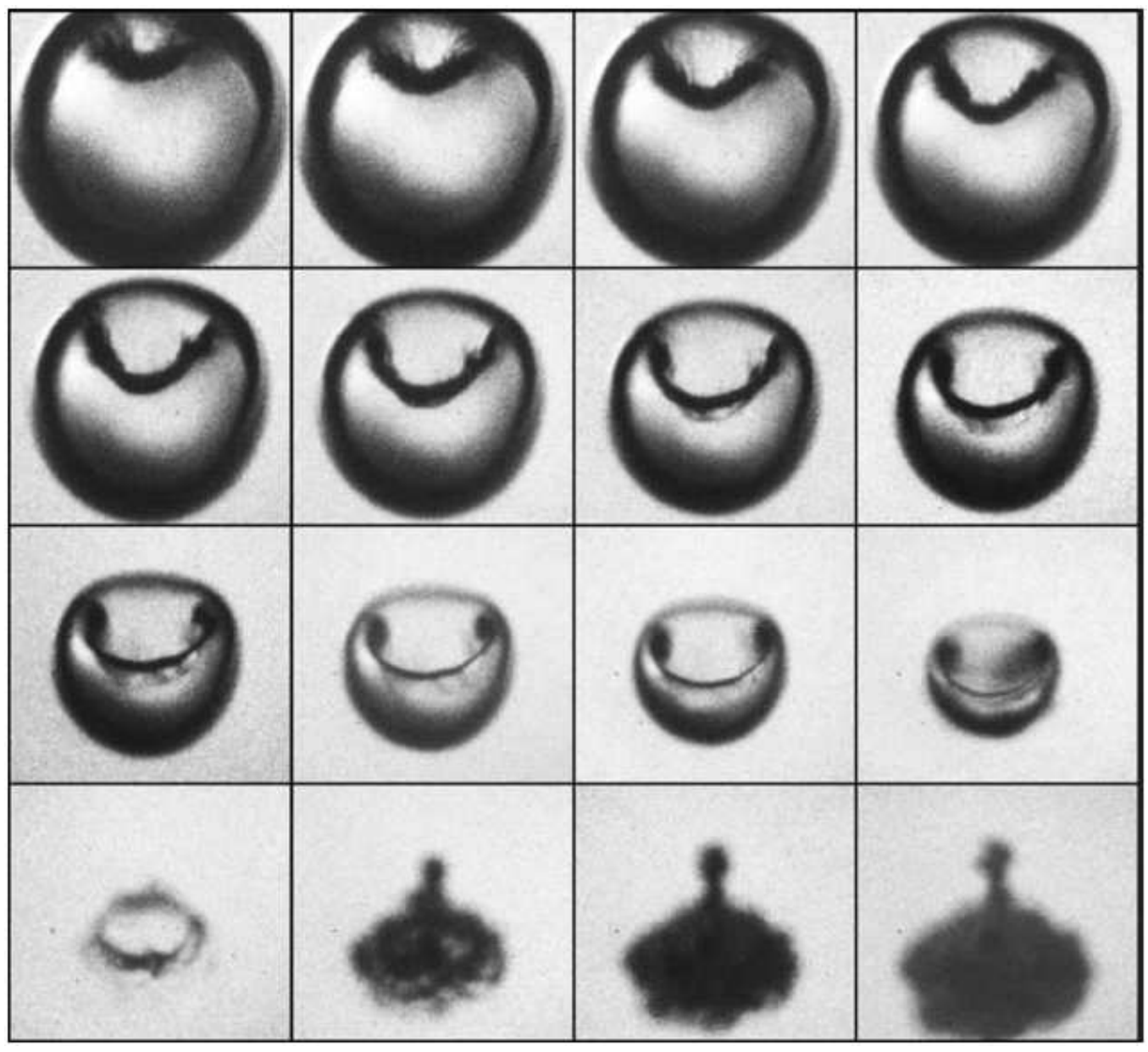

Figure 1.6: Collapse of a laser-generated cavitation bubble near a solid surface (maximum radius: $1.5 \mathrm{~mm}$; initial stand-off distance: $2.4 \mathrm{~mm}$; interframe spacing: $1 \mu \mathrm{s})$. Reprinted with permission from Lindau \& Lauterborn (2003). Copyright 2003 by Cambridge University Press.

bubbles or gas nuclei (Philipp et al., 1993; Ohl \& Ikink, 2003; Sankin et al., 2005), as illustrated by Figure 1.7. When non-spherical bubble collapse takes place near the kidney stone, the re-entrant jet may directly impact the stone. This phenomenon has been regarded as the most important cause for cavitation damage in SWL (Coleman et al., 1987; Crum, 1988) and has been studied in the context of cavitation erosion (Philipp \& Lauterborn, 1998) as well. Two-dimensional shockbubble interactions have been studied in the framework of shock propagation in 
non-uniform media: Dear \& Field (1988) and Bourne \& Field (1992) considered the impact of shockwaves onto cavities in a water/gelatin mixture to better understand ignition and propagation of explosive reactions, while Haas \& Sturtevant (1987) investigated the impingement of shockwaves onto gaseous cylinders.

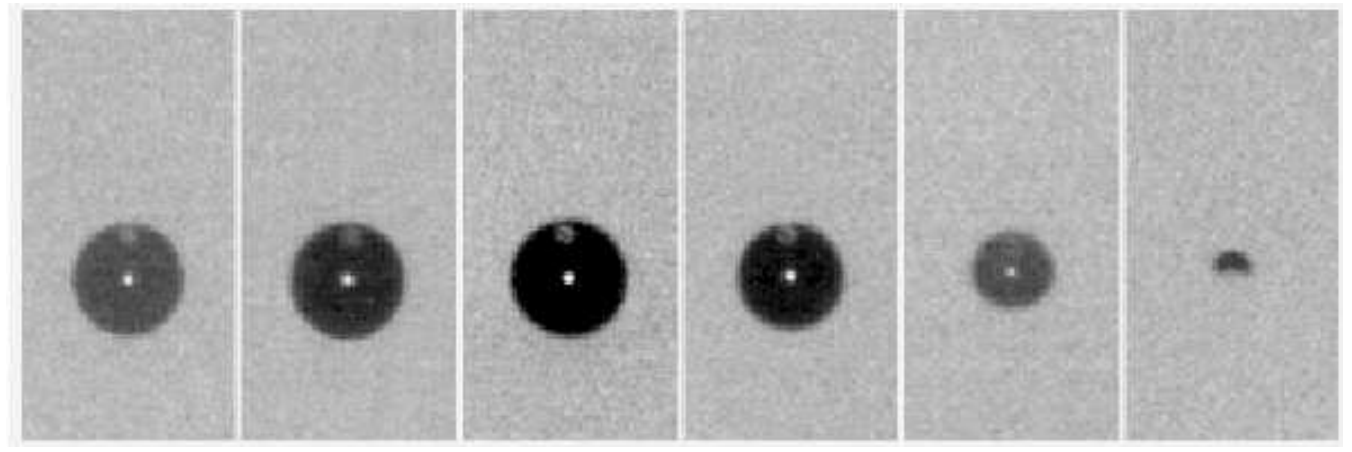

Figure 1.7: Shock-induced collapse of a laser-generated cavitation bubble in a freefield (frame width: $0.7 \mathrm{~mm}$; interframe spacing: $0.5 \mu \mathrm{s}$ ). Reprinted with permission from Sankin et al. (2005). Copyright 2005 by the American Institute of Physics.

\subsubsection{Numerical simulations}

Because of the complexity of the physics and the wide range of spatial and temporal scales present in bubble collape, it is difficult to perform precise measurements in experiments. Numerical simulations have therefore emerged as a reliable tool to complement analytical and experimental studies. However, a direct numerical simulation of such flow phenomena is not realizable at the present time, because of high computational costs and a lack of suitable algorithms. Although simulations of single-bubble collapse have been carried out over last few decades, prior studies usually fell into one of two categories: the numerical method accurately solved a problem in which the physics were highly restrictive, or the physics of the problem were more adequately represented at the expense of accuracy.

Methods based on potential theory were first used to compute the collapse of a bubble near a wall (e.g., the Particle In Cell Method of Plesset \& Chapman (1971), the Boundary Integral Method (BIM) of Blake et al. (1986) and Best (1993), and 
later the Boundary Element Method of Klaseboer et al. (2006)). These methods capture the initial bubble behavior and the formation of the re-entrant jet well, so that quantities of practical use can be computed, such as the collapse time, the jet speed, or the bubble pressure. However, during the last stages of collapse, the bubble wall velocity becomes comparable to the local sound speed, so that the assumption of liquid incompressibility breaks down. Additional drawbacks of these methods include the fact that shockwaves cannot be simulated in an incompressible formulation and that the bubble pressure is assumed uniform. Furthermore, "numerical surgery" (Best, 1993) must be carried out after the jet has hit the distal side, in order to correct for the topological change in the bubble surface. Another study based on incompressible flow was undertaken by Popinet \& Zaleski (2002), who used an axisymmetric front-tracking method to investigate the effect of viscosity for Rayleigh collapse near a solid surface. This method has drawbacks similar to the BIM.

However, in the problems under consideration, compressibility accounts for a large part of the energy dissipation (Vogel et al., 1988), which is important for shock emission upon collapse and is therefore relevant to the damage potential. Recent efforts have therefore been devoted to developing methods capable of simulating the full compressible Euler equations in order to capture any shock generated during the process; most of these methods are in their verification and validation stages. The main difficulty consists of treating shockwaves and interfaces in a robust and stable fashion. Thus, in order to handle these features, shockcapturing methods solving the Euler equations are currently being developed. Ball et al. (2000) used a two-dimensional second-order accurate Free-Lagrange Method to study shock-bubble interactions based on the experiments of Bourne \& Field (1992). The gas was observed to heat substantially due to internal reflections of the shockwave. However, the complexities of the mesh are not very practical for intricate bubble shapes in three dimensions. Nourgaliev et al. (2006) employed adaptive characteristics-based matching, and $\mathrm{Hu}$ et al. (2006) used a level set method with corrections to account for conservation losses to simulate the same problem and 
two-dimensional underwater explosions, with similar results. However, the methods are not conservative near interfaces because the numerical fluxes do not have the telescoping property (LeVeque, 1992). Chang \& Liou (2007) implemented a volume fraction formulation in the $\mathrm{AUSM}^{+}$-up methodology to simulate similar problems. The main drawback of this method is that several problem-dependent constants must be adjusted. The Rayleigh collapse of a bubble was studied by Nagrath et al. (2006), who combined a finite element and a level set method to simulate three-dimensional collapse of a spherical bubble initially following the Rayleigh-Plesset equation for a single set of parameters. The most comprehensive work to date in the study of shock-bubble interactions in SWL was carried out by Jamaluddin (2005) using an axisymmetric implementation of the Free-Lagrage Method of Ball et al. (2000). The shockwave resulting from the impact of the jet onto the distal side was captured, and predictions for jet speed and wall pressure were made for certain specific parameters.

\subsection{Motivation, accomplishments and overview}

As illustrated in this chapter, bubble collapse shows tremendous potential for damage. Since cavitation is such a ubiquitous phenomenon, a better understanding of cavitation erosion is of great interest in numerous applications, including SWL. However, the complexity of the physics and the wide range of spatial and temporal scales limit experiments, while current numerical simulations do not represent the physics accurately.

The motivation for the present research lies in the recognition that numerical simulations of single-bubble collapse will provide insights into cavitation damage in general. Furthermore, a full understanding of the role of cavitation in SWL will allow for improvements in the treatment techniques (e.g., lithotripter design). The objectives of the present thesis are twofold. The first is to develop a robust numerical method that accurately computes flows in which strong shockwaves and interfaces separating fluids with high density ratios (e.g., water and air) interact. 
The second is to utilize this method to simulate the collapse of a gas bubble, in order to investigate non-spherical bubble dynamics and to provide quantitative measurements for the potential damage generated by bubble collapse near a solid surface. These results can then be cast in the context of SWL to determine the role of bubble collapse in stone comminution.

The work reported in this thesis constitutes the most comprehensive computational study of non-spherical bubble collapse to date, in terms of the range of the parameter space, of the accuracy and robustness of the numerical method, and of the detailed analysis of the results. Since the entire flow field is computed, quantitative measurements can be readily made, and the relevant physical phenomena can be studied (e.g., shock-bubble interaction, generation of shockwaves during collapse, and interactions between shockwaves and interfaces or solid surfaces). These results are then examined more carefully in the context of shockwave lithotripsy.

The main contributions of the current research to the field of numerical methods in bubbles dynamics include:

- The development of a new high-order accurate, quasi-conservative, interfaceand shock-capturing scheme for the simulation of compressible multicomponent flows with shocks (Chapter 3),

- Robust and stable simulations of strong shockwaves and interfaces between fluids of very large density ratios,

- Verification and validation of the method,

- Extension of the method to cylindrical coordinates and to non-uniform grid spacing,

- Theoretical investigations of non-spherical bubble collapse (Chapter 4),

- Numerical simulations of Rayleigh collapse, driven by the initial pressure difference across the interface, and of shock-induced collapse, driven by the passage of a shockwave (Chapter 4), 
- Characterization of the dynamics of non-spherical bubble collapse,

- Analysis of the mechanism of formation of the re-entrant jet in shockinduced collapse and in Rayleigh collapse near a wall,

- Determination of the shockwaves emitted upon bubble collapse,

- Estimation of the bubble pressure and energy radiated during collapse,

- Quantitative measurements of the pressure generated by bubble collapse along a neighboring wall,

- Application of the numerical method to SWL conditions (Chapter 5),

- Analysis of the influence of the pulse properties on the bubble dynamics,

- Evaluation of the potential damage caused by bubble collapse in SWL,

- Presentation of shock-induced collapse as a stone comminution mechanism,

- Implementation of the present results as boundary conditions for a code to simulate elastic wave propagation in kidney stones to investigate the internal structural damage due to the pressure generated by bubble collapse (Chapter $5)$.

The thesis is organized in the following manner. Chapter 2 provides the background information for the rest of the work. First, the problem is defined. Then, relevant assumptions are listed and the governing equations are presented; additional relationships pertaining to the equation of state are included in Appendix A. Chapter 3 is devoted to the numerical scheme. The method is described in detail for a uniform Cartesian grid and then extended to cylindrical coordinates; further derivations for non-uniform grids are listed in Appendix B. The verification of the method is presented using various test problems. The dynamics of non-spherical bubble collapse are then studied in detail in Chapter 4. Theoretical remarks on spherical bubble dynamics are first provided, followed by flow visualizations of the simulations. After investigating the dynamics of collapse, the jet formation 
mechanism is discussed. Then, the damage potential of bubble collapse is examined. The methodology is extended to SWL in Chapter 5, where the modeling of the pulse is first considered, followed by a parameter study of bubble collapse in SWL. A comminution mechanism for small- to medium-sized stone is then presented. Finally, Chapter 6 provides concluding remarks and further extensions for the present work. 


\section{Chapter 2}

\section{Physical model}

In the current chapter, the physical model used to study bubble collapse is presented. First, a description of the occurrence of single-bubble collapse in shockwave lithotripsy is provided and the set-up of the problem is presented. Then, relevant assumptions are discussed to motivate the use of the Euler equations for studying compressible multicomponent flows. The stiffened equation of state and the advection equations required to close the system are then presented and the range of parameters explored in the study is listed.

\subsection{Problem description}

\subsubsection{Single-bubble collapse in shockwave lithotripsy}

In shockwave lithotripsy (SWL), the typical cycle of the lifetime of a bubble is summarized in Figure 2.1. A pre-existing gas nucleus or vapor bubble is initially in equilibrium with its surroundings. When the compressive part of the lithotripter pulse impacts the bubble, it induces collapse. Then, because of the tensile portion of the pulse, the bubble cavitates and grows to a large size. At its maximum radius, the bubble mainly contains vapor. It then undergoes an isolated collapse due to the difference between the bubble pressure and its surroundings. After several of these cycles, the bubble may contain more non-condensible gas because of rectified diffusion (Hsieh \& Plesset, 1961; Crum, 1980), so that its equilibrium 
radius is larger.

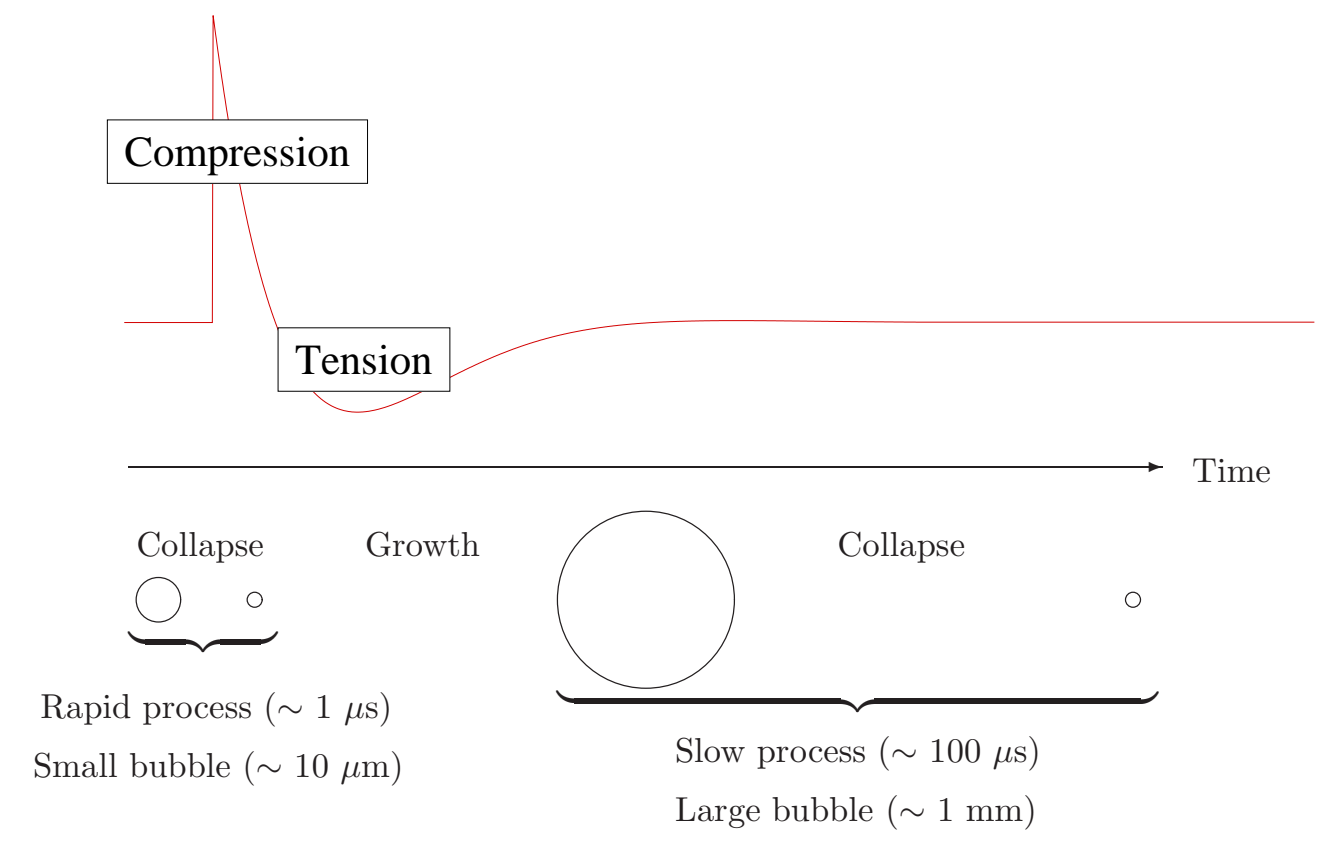

Figure 2.1: Typical bubble cycle in SWL.

The first type of collapse caused by the shockwave is termed shock-induced collapse (SIC). This phenomenon is usually rapid, because the initial bubble radius is small $\left(R_{o} \approx 10 \mu \mathrm{m}\right)$ and the pressure ratio is large $\left(p_{s} / p_{o}=353\right.$ for a lithotripter pulse, $\left.p_{B} \approx p_{a t m}\right)$. The resulting collapse time is on the order of microseconds. The second type of collapse caused by the static pressure difference between the bubble and its surroundings is called Rayleigh collapse (RC). ${ }^{1}$ This phenomenon lasts over a longer time-scale, because the initial bubble radius is larger $\left(R_{o} \approx 1\right.$ $\mathrm{mm})$ and the pressure ratio is smaller $\left(p_{s} / p_{o}=34, p_{B} \approx p_{v}\right.$ and $\left.p_{o}=p_{a t m}\right)$. The resulting collapse time is on the order of milliseconds.

In order to understand the fundamental bubble dynamics in SWL, the Rayleigh collapse and shock-induced collapse described above are studied in depth. Further-

\footnotetext{
${ }^{1}$ Named after the original problem considered by Rayleigh (1917).
} 
more, the limiting case of shock-induced collapse for which the shock speed in the liquid is infinite corresponds to a Rayleigh collapse. In order to provide a bridge for comparing the two problems, this third problem, which is equivalent to raising the ambient pressure by a giving amount while keeping the bubble pressure at atmospheric value, is considered. Thus, three fundamental problems are considered:

1. Rayleigh collapse under cavitation conditions $\left(p_{s} / p_{o} \approx 34\right)$,

2. Shock-induced collapse $\left(p_{s} / p_{o}=353\right)$, and

3. Rayleigh collapse under shock conditions $\left(p_{s} / p_{o}=353\right){ }^{2}$

These three problems are considered in two different geometries: free-field collapse, in which the domain consists of an infinite sea of liquid and collapse near a wall, in which the domain is half-infinite, with a perfectly reflecting solid surface extending to infinity. The presence of a wall introduces an additional length scale, i.e., the stand-off distance.

\subsubsection{Domain}

The domain consists of a cylinder, along whose centerline the bubble is located. Appropriate non-reflecting boundary conditions are used at the edge of the cylinder to extend the domain to infinity, as discussed in Section 3.5. Initially, the bubble is spherical and has radius $R_{o}$. It contains only non-condensible gas and and is assumed to be in equilibrium with its surroundings. ${ }^{3}$ If the collapse takes place near a wall, the initial stand-off distance is denoted $H_{o}$. The bubble radius, $R(t)$, and the stand-off distance, $H(t)$, change throughout the collapse. Because most of the problems considered involve non-spherical collapse, $R$ is an average value calculated from the bubble volume, which is given by Equation C.2, while $H$ denotes the position of the bubble centroid, as described by Equation C.4.

\footnotetext{
${ }^{2}$ If the collapse occurs near a solid boundary, $p_{s} / p_{o}=714$, to account for pressure doubling caused by the reflection of the shock.

${ }^{3}$ Because the focus is on the potential for damage, the nucleation and growth phases are ignored; in Rayleigh collapse, this implies that the initial bubble radius is $R_{o}=R_{\max }$.
} 
A slice through the center of the domain is shown in Figure 2.2, where the dashed line denotes the computational domain. The region enclosed by the dashed line is revolved about the axis, $r=0$ (bottom of the domain). In the case of shock-induced collapse, the incoming shockwave starts inside the domain, travels from right to left, and impinges the wall normally. Thus, the problems of interest (Rayleigh collapse and shock-induced collapse in a free-field and near a wall) are axisymmetric, so that they can be reduced from three spatial dimensions to cylindrical coordinates with azimuthal symmetry. Hence, each computational cell consists of an annular volume. This simplification greatly alleviates the computational expense.

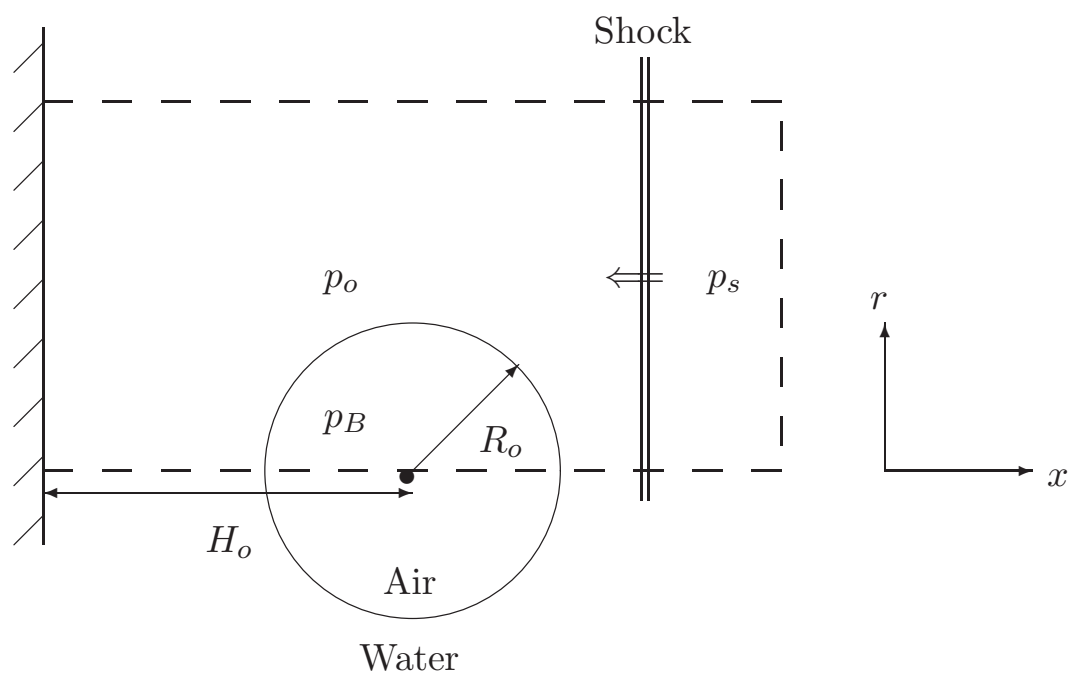

Figure 2.2: Schematic of the problem geometry.

The pressure in three regions is specified: the bubble pressure, $p_{B}$, the ambient pressure, $p_{o}$, and the shock pressure, $p_{s}$. If there is no shock, $p_{s}=p_{o}$. In the present set-up, the proximal side always denotes the right side of the bubble in Figure 2.2 (i.e., the side closest to the shock), and the distal side always denotes the left side of the bubble (i.e., the side closest to the wall). 
In SWL, the pulse consists of a steep compressive front, followed by a long expansion. Thus, an additional length (or time) scale is introduced to the problem, e.g., the pulse width. In order to simplify the analysis, shockwaves consist of a step change in pressure in the presentation of the results for shock-induced collapse in Chapter 4. When applying the results to SWL in Chapter 5, a suitable lithotripter pulse with properties similar to that of Figure 1.4 is used.

In practice, the shock is not necessarily aligned with the stone normal and the stone surface may not be flat, thus illustrating the three-dimensionality of the general problem. The present assumption of axisymmetry constitutes the geometrical configuration for which the bubble collapse is the most energetic, because of symmetry. A full three-dimensional simulation would be necessary to compute the general problem, in which case an additional parameter, the angle between the shock and the wall normal, should be introduced. Furthermore, the bubble collapse may take place under different conditions as well in SWL. For instance, the collapse of each bubble generates shockwaves that may interact with bubbles out of equilibrium, so that both the amplitude of the shock during the interaction and the bubble radius may vary with time and space. However, these issues reside beyond the scope of the present work.

\subsection{Equations of motion}

\subsubsection{Compressible multicomponent flows}

The main characteristics of single-bubble collapse in shockwave lithotripsy are large non-spherical interface deformations and the generation and propagation of shockwaves. Interactions between different types of waves and interfaces are thus the most important flow features, so that compressibility effects in gases and liquids are primordial.

On the other hand, diffusive effects, surface tension, and mass transfer are not expected to play a significant role until the rebound and subsequent growth, as the collapse time is small compared to the other time scales. This is illustrated 
in the following rudimentary analysis of spherical collapse. Because the bubble wall reaches speeds comparable to the sound speed in water, $c_{L}$, at collapse, the characteristic speed is taken as $c_{L}$, so that the Mach number is unity. The Reynolds number for the present problem can be defined as $R e=R_{o} c_{L} / \nu_{L}$, while the Weber number can be expressed as $W e=\rho_{L} R_{o} c_{L}^{2} / S$. Room temperature values of the viscosity and surface tension are taken $\left(\nu_{L}=10^{-6} \mathrm{~m}^{2} / \mathrm{s}, S=0.0728 \mathrm{~N} / \mathrm{m}\right)$. The Prandtl and Schmidt numbers depend on the ambient temperature, with $\operatorname{Pr} \approx 6, S c \approx 450$. In order to assess their importance during spherical collapse, the magnitude of surface tension and viscous effects can be studied using the Rayleigh-Plesset equation 4.3 discussed in Section 4.1.1. A comparison of the magnitude of the contributions of these effects with those of the bubble pressure and inertia are shown in Figure 2.3, for $p_{s} / p_{o}=34$ and $R_{o}=10^{-6}, 10^{-3} \mathrm{~m}$. The length scales are non-dimensionalized using the initial bubble radius. The main contribution to the acceleration is the term consisting of the bubble pressure and the velocity, whereas the viscous and surface tension effects are essentially negligible. In non-spherical collapse, the jet is characterized by high curvatures and small scales, so that viscous and surface tension effects may be important for small bubbles in shock-induced collapse, especially when the jet develops, as explained in Section 4.3.6.

Based on these assumptions, compressible multicomponent flows are considered (Shyue, 1998). They constitute a subset of multiphase flows where the different fluid components, characterized by their respective (constant) ratio of specific heats, are immiscible. The resulting inviscid and adiabatic flows are governed by the Euler equations. The bubble contains only non-condensible gas (e.g., air), which is assumed to behave ideally; the liquid is water and obeys an appropriate equation of state. 

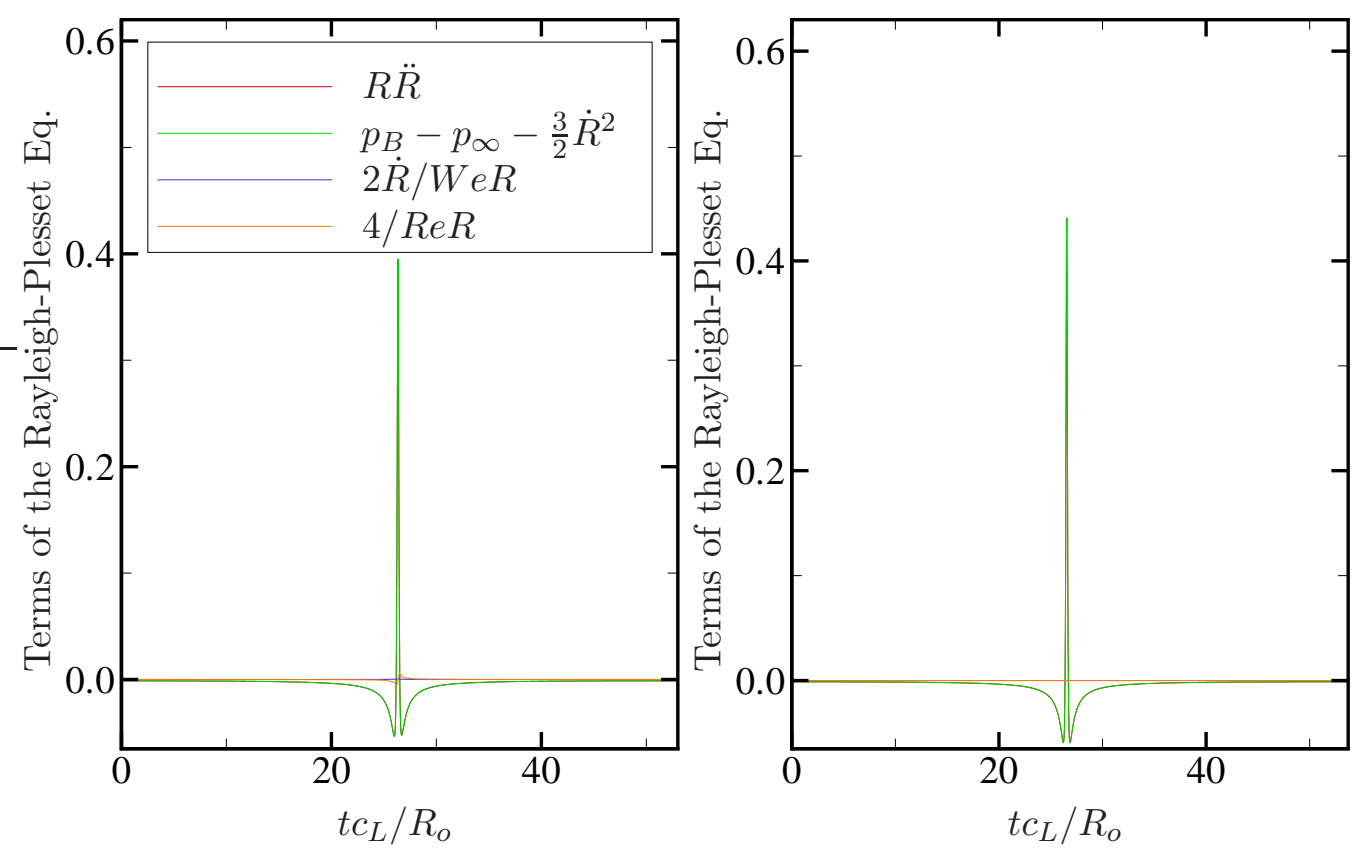

Figure 2.3: Solution to the Rayleigh-Plesset equation for one period $\left(p_{s} / p_{o}=34\right)$. Left: $R_{o}=1 \mu \mathrm{m}$; right $R_{o}=1 \mathrm{~mm}$.

\subsubsection{The Euler equations}

Compressible multicomponent flows are governed by the unsteady Euler equations, written here in conservative form,

$$
\frac{\partial \boldsymbol{q}}{\partial t}+\nabla \cdot \boldsymbol{f}=\mathbf{0}, \quad \boldsymbol{q}(\mathbf{x}, t)=\left(\begin{array}{c}
\rho \\
\rho \mathbf{u} \\
E
\end{array}\right), \quad \mathbf{f}(\mathbf{q})=\left(\begin{array}{c}
\rho \mathbf{u} \\
\rho \mathbf{u u}+p \mathbf{I} \\
(E+p) \mathbf{u}
\end{array}\right)
$$

where $\rho$ is the density, $\mathbf{u}$ is the velocity vector, $p$ is the pressure, $E$ is the total energy and $\mathbf{I}$ is the identity tensor. An equation of state relating the pressure and the internal energy is required to close the system, as described in Section 2.2.3. Given an appropriate set of initial and boundary conditions, the resulting system can then be solved.

In the simulations of bubble collapse, cylindrical coordinates with azimuthal 
symmetry are used:

$$
\left(\begin{array}{c}
\rho \\
\rho u \\
\rho v \\
E
\end{array}\right)_{t}+\left(\begin{array}{c}
\rho u \\
\rho u^{2}+p \\
\rho u v \\
(E+p) u
\end{array}\right)_{x}+\left(\begin{array}{c}
\rho v \\
\rho u v \\
\rho v^{2}+p \\
(E+p) v
\end{array}\right)_{r}+\frac{1}{r}\left(\begin{array}{c}
\rho v \\
\rho u v \\
\rho v^{2} \\
(E+p) v
\end{array}\right)=\mathbf{0}
$$

The axial direction is represented by the $x$-coordinate. Equations 2.2 are then discretized in a finite volume formulation, as explained in Section 3.7.1.

In the numerical scheme, the characteristic form of the Euler equations is exploited. The characteristic equations are written here for the $x$-direction only:

$$
\frac{\partial \mathbf{w}}{\partial t}+\Lambda \frac{\partial \mathbf{w}}{\partial x}=0
$$

where

$$
d \mathbf{w}=\left(d u-\frac{d p}{\rho c}, d \rho-\frac{d p}{c^{2}}, d v, d u+\frac{d p}{\rho c}\right)^{T}, \quad \Lambda=\operatorname{Diag}[u-c, u, u, u+c]
$$

This form is necessary for the non-reflecting boundary conditions and the characteristic reconstruction, both described in Chapter 3. During the numerical reconstruction of the variables, the primitive variables are projected onto the characteristic fields. To do so, the following transformation matrices are required:

$$
L=\left(\begin{array}{cccc}
0 & -\frac{1}{2} & 0 & \frac{1}{2 \rho c} \\
1 & 0 & 0 & -\frac{1}{c^{2}} \\
0 & 0 & 1 & 0 \\
0 & \frac{1}{2} & 0 & \frac{1}{2 \rho c}
\end{array}\right), \quad R=\left(\begin{array}{cccc}
\frac{\rho}{c} & 1 & 0 & \frac{\rho}{c} \\
-1 & 0 & 0 & 1 \\
0 & 0 & 1 & 0 \\
\rho c & 0 & 0 & \rho c
\end{array}\right)
$$




\subsubsection{The stiffened equation of state}

Because the fluids consist of liquids and gases, an appropriate equation of state must be chosen. The stiffened equation of state (Harlow \& Amsden, 1971),

$$
\Gamma p+\Pi_{\infty}=E-\frac{1}{2} \rho \mathbf{u} \cdot \mathbf{u},
$$

is used to close the Euler equations, where $\Gamma=1 /(\gamma-1)$ and $\Pi_{\infty}=\gamma P_{\infty} /(\gamma-1)$.

For perfect gases, $\gamma$ is the ratio of specific heats and $P_{\infty}=0$, so that the usual relationship between the pressure and the internal energy is achieved. For water, as shown by Equations A.29 and A.30, $\gamma$ and $P_{\infty}$, which is a measure of the stiffness of the liquid, are determined based on the shock Mach number (Cocchi et al., 1996). Because the shock Mach numbers in water relevant to SWL are close to unity, the limit $M_{s} \rightarrow 1$ is taken, so that constant values of $\gamma_{L}=6.684$ and $P_{\infty}=4049 \mathrm{~atm}$ are employed for all the simulations. Further relations derived from the stiffened equation of state are provided in Appendix A. This equation of state is thermodynamically consistent (Menikoff \& Plohr, 1989) and has been used extensively to model multicomponent flows with shockwaves (Cocchi et al., 1996; Shyue, 1998; Saurel \& Abgrall, 1999b,a; Abgrall \& Karni, 2001). However, whether the equation is thermodynamically consistent when the interface diffuses numerically is unclear. It should be noted that in the limit $\Delta x \rightarrow 0$ the interface converges to a sharp one, leading to an appropriate physical behavior.

Although the sound speed does not appear explicitly in the Euler equations 2.1 , it is used for upwinding in the numerical algorithm. Given the equation of state 2.6, the local speed of sound can be computed, as shown in Equation A.9 Appendix A:

$$
c=\sqrt{\gamma \frac{p+P_{\infty}}{\rho}} .
$$

\subsubsection{Advection equations}

Since mass transfer is neglected, interfaces between two fluid components are advected by the flow. Because the fluid components are assumed immiscible, in- 
terfaces are specified by a discontinuity in the fluid composition characterized by the material properties $\gamma$ and $P_{\infty}$. Thus, the functions $\phi=\left(\Gamma, \Pi_{\infty}\right)^{T}$ obey the advection equation,

$$
\frac{\partial \phi}{\partial t}+\boldsymbol{u} \cdot \nabla \boldsymbol{\phi}=\mathbf{0}
$$

Although not fully conservative, the system of equations 2.1 and 2.8 conserves the required physical properties: the Euler equations 2.1 are conservative, ensuring that mass, momentum and energy are conserved, while the advection equations 2.8 , which are non-conservative, specify the correct location of the interface, so that the relevant properties are defined. Equations 2.1 and 2.8 form a quasi-conservative system of equations (Abgrall, 1996).

The advection equations 2.8 could be combined with the continuity equation to form the following conservative equations:

$$
\frac{\partial}{\partial t}(\rho \phi)+\nabla \cdot(\rho \mathbf{u} \phi)=0
$$

where $\phi$ could be any function of $\gamma$ and $P_{\infty}$. The system of equations 2.1 and 2.9 is fully conservative. However, it is shown in Section 3.4.1 that this coupling leads to oscillations at interfaces when using standard shock-capturing schemes, so that the functions $\Gamma=1 /(\gamma-1)$ and $\Pi_{\infty}=\gamma P_{\infty} /(\gamma-1)$ must be advected (according to Equation 2.8).

\subsubsection{Non-dimensionalization and physical parameters}

The ambient density and sound speed of water, $\rho_{L}$ and $c_{L}$, along with the initial bubble radius, $R_{o}$, are used to non-dimensionalize the equations. The density and sound speed are based on the values used in the derivation of the stiffened equation of state (Cocchi et al., 1996). Table 2.1 lists the relevant material properties of air and water.

The problems under consideration are simplified in order to minimize the number of parameters governing the physics of the problem. From the equations of motion and the problem description, the following non-dimensional groups can be 
Table 2.1: Fluid properties.

\begin{tabular}{l|cc} 
Variable & Dimensional value & Non-dimensional value \\
\hline$\rho_{G}$ & $1.22 \mathrm{~kg} / \mathrm{m}^{3}$ & $1.22 \times 10^{-3}$ \\
$\rho_{L}$ & $998 \mathrm{~kg} / \mathrm{m}^{3}$ & 1.00 \\
$c_{G}$ & $341 \mathrm{~m} / \mathrm{s}$ & 0.21 \\
$c_{L}$ & $1650 \mathrm{~m} / \mathrm{s}$ & 1.00 \\
$\gamma_{G}$ & - & 1.40 \\
$P_{\infty, G}$ & $0.00 \mathrm{~atm}$ & 0.00 \\
$\gamma_{L}$ & - & 6.68 \\
$P_{\infty, L}$ & $4050 \mathrm{~atm}$ & 0.15
\end{tabular}

formed: $H_{o} / R_{o}, p_{s} / p_{o}$ or $p_{o} / p_{B}, p_{o} / P_{\infty, L}, \rho_{G} / \rho_{L}, \gamma_{L}, \gamma_{R}$. However, $p_{o} / P_{\infty, L} \ll 1$, and, although $\gamma$ varies within the interfacial region due to numerical diffusion, it is bound by $\gamma_{L}$ and $\gamma_{R}$. Therefore, three non-dimensional numbers ${ }^{4}$ govern the problems under consideration:

1. The pressure ratio across the shock, $p_{s} / p_{o}$, for shock-induced collapse or across the interface, $p_{o} / p_{B}$, for Rayleigh collapse, ${ }^{5}$

2. The initial stand-off distance from the wall, $H_{o} / R_{o}$, and

3. The density ratio across the interface, $\rho_{G} / \rho_{L}$.

The pressure ratio is varied in the range, $p_{s} / p_{o}=34-710$. The lower bound corresponds to the pressure ratio driving the collapse of a cavitation bubble $\left(p_{a t m} / p_{v} \approx 34\right)$. In the present work, the bubble initially contains a fixed amount of non-condensible gas, because phase change is neglected; in nature, a cavitation bubble about to undergo Rayleigh collapse contains mainly vapor at its maximum radius and only a small amount of non-condensible gas. As explained in Section

\footnotetext{
${ }^{4}$ In the case of SWL, an additional non-dimensional parameter, the normalized pulse width $\left(\sigma / R_{o}\right)$, enters the problem.

${ }^{5}$ Alternately, a Mach number, $M_{s}$, based on the pressure ratio can be used, as shown in Equation A.25.
} 
4.1.1, the collapse is arrested by the compression of the non-condensible gas. Since it contains much more gas in the simulations, the bubble cannot collapse to as small a volume and reach as high an interfacial velocity as in nature. As a result of this modeling, Rayleigh collapse under cavitation conditions is much gentler in the simulations than it is in nature. The upper bound corresponds to an explosion occurring near the bubble (Cole, 1948). ${ }^{6}$

Based on preliminary tests, the effect of the wall on the results is minor for initial stand-off distances greater than five initial radii. Thus, the range, $H_{o} / R_{o}=$ $1.05-5$ is considered. Values less than unity are considered in experiments (Tomita \& Shima, 1986; Vogel et al., 1989; Philipp \& Lauterborn, 1998). However, because the bubble is not spherical in such a situation, the amount of vapor at $R_{\max }$ is not the same, so that it is not clear whether meaningful comparisons can be made.

Finally, the present work focuses on air bubbles in water. Thus, the density ratio is held fixed at $\rho_{G} / \rho_{L}=1.22 / 998$, except in Section 4.3 .9 where the bubble density is varied in order to study the bubble circulation.

\subsection{Summary of the physical model}

Rayleigh collapse and shock-induced collapse of an initially spherical air bubble in a free-field and near a wall are investigated. These types of collapse are fundamental problems in shockwave lithotripsy, as the former is exemplified by the isolated collapse of a cavitation bubble, while the latter involves the impact of an incoming shock onto a pre-existing bubble. The problems are defined in Table 2.2 based on the pressure in the different regions shown in Figure 2.2. RC refers to "Rayleigh collapse", SIC to "shock-induced collapse" and W to "collapse near a wall". It should be noted that the case for which the shock pressure and the ambient pressure are equal implies no shock (i.e., Rayleigh collapse). The sub-problems listed in the second row of the RC cases are considered as comparison tools, because they

\footnotetext{
${ }^{6}$ Although the pressure ratios are large, the corresponding Mach numbers are close to unity, as observed in Equation A.25.
} 
do not generally occur in nature.

Table 2.2: Definition of the different problems under consideration.

\begin{tabular}{|c|c|c|c|c|}
\hline $\begin{array}{l}\text { Collapse } \\
\text { type }\end{array}$ & $\begin{array}{l}\text { Initial } \\
\text { stand-off } \\
\text { distance }\end{array}$ & $\begin{array}{l}\text { Shock } \\
\text { pressure } \\
\left(p_{s}\right)\end{array}$ & $\begin{array}{l}\text { Ambient } \\
\text { pressure } \\
\left(p_{o}\right)\end{array}$ & $\begin{array}{l}\text { Bubble } \\
\text { pressure } \\
\left(p_{B}\right)\end{array}$ \\
\hline \multirow[t]{2}{*}{$\mathrm{RC}$} & \multirow{2}{*}{$\infty$} & $p_{a t m}$ & $p_{\text {atm }}$ & $p_{v} \ldots$ \\
\hline & & $p_{\max }$ & $p_{\max }$ & $p_{a t m}$ \\
\hline SIC & $\infty$ & $p_{\max }$ & $p_{a t m}$ & $p_{a t m}$ \\
\hline \multirow{2}{*}{ RCW } & \multirow{2}{*}{$H_{o}$} & $p_{a t m}$ & $p_{a t m}$ & $p_{v}$ \\
\hline & & $p_{\max }$ & $p_{\max }$ & $p_{a t m}$ \\
\hline SICW & $H_{o}$ & $p_{\max }$ & $p_{a t m}$ & $p_{a t m}$ \\
\hline
\end{tabular}

The problems are idealized, so that the underlying physics of bubble collapse can be probed. Because the main features of these flows involve interaction between various types of waves and interfaces, compressible multicomponent flows are considered, where diffusive effects, surface tension, and mass transfer are neglected, and the fluid components are immiscible. Hence, the axisymmetric Euler equations, closed by a stiffened equation of state and supplemented by appropriate advection equations, are solved. For the present study of Rayleigh collapse and shock-induced collapse, three non-dimensional numbers are expected to govern the physics: $H_{o} / R_{o}, p_{s} / p_{o}$, and $\rho_{G} / \rho_{L}$. In the case of shockwave lithotripsy, the normalized pulse width, $\sigma / R_{o}$, also enters the problem. 


\section{Chapter 3}

\section{Numerical method}

A new numerical framework is developed to solve compressible multicomponent flow problems. ${ }^{1}$ The need for the development of a new method is first motivated. Then, the time-marching, the spatial discretization, and the interface-capturing schemes are described, followed by a presentation of the boundary conditions. Thereafter, the method is validated. The chapter ends with the extension of the method to cylindrical coordinates and a discussion of the limitations of inviscid simulations.

\subsection{Numerical simulations of interfaces and shockwaves}

Previous numerical studies of bubble dynamics can be divided into two main categories: incompressible and compressible simulations. The former is a mature field, where several different techniques, such as Boundary Integral (Power, 1995), Level Set (Sethian, 1996), and Particle (Koumoutsakos, 2005) methods, are capable of accurately describing the dynamics of interfaces for certain types of flows. On the other hand, the study of compressible multiphase flows, especially when shockwaves are present, is still in its early developmental stages. In the present work, a

\footnotetext{
${ }^{1}$ The corresponding journal article presenting the two-dimensional method solving the system of equations 2.1 and 2.8 on a uniform Cartesian grid was published in the Journal of Computational Physics (Johnsen \& Colonius, 2006), Copyright Elsevier 2006.
} 
numerical method is developed to simulate compressible multicomponent flows in a first step to study the compressible-flow features of bubble dynamics.

\subsubsection{Shock capturing}

Because the Euler equations 2.1 are nonlinear hyperbolic conservation laws, a discontinuous solution can develop in a finite time even with smooth initial conditions. Thus, shock-capturing schemes, in which numerical dissipation is added to prevent oscillations near shockwaves and which result in the smearing of discontinuities over a few grid points, form the backbone of numerical methods simulating compressible multicomponent flows. Shock-capturing schemes that exhibit up to second-order accuracy are widely used in computational fluid dynamics to resolve compressible flow features that involve both shockwaves and complex smooth structures. In order to achieve higher-order accuracy in smooth regions, finite difference and finite volume Essentially Non-Oscillatory (ENO) and Weighted ENO (WENO) methods can be used and perform well in such problems (Harten et al., 1987; Shu \& Osher, 1988, 1989; Liu et al., 1994; Jiang \& Shu, 1996). They prevent oscillations near shockwaves without introducing excessive dissipation, which is a serious drawback of first- and second-order accurate methods (Shu, 2003). However, a naive implementation of the aforementioned schemes in compressible multicomponent flow problems gives rise to oscillations at material interfaces. Thus, the interface must be treated in a special fashion.

\subsubsection{Interface tracking and capturing}

In early algorithms for computing compressible multicomponent flows, the discontinuous nature of the fluid composition was represented by the mass fraction (Larrouturou \& Fezoui, 1989), the ratio of specific heats (Karni, 1994), or a levelset function (Mulder et al., 1992), and evolved according to an advection equation coupled to the Euler equations. The resulting system was solved using first- and second-order accurate reconstructions with a Roe solver (Roe, 1981). However, spurious oscillations developed at interfaces. The cause of these oscillations was 
identified by Abgrall (1996), who proposed a quasi-conservative method based on the mass fraction formulation for gases; subsequently, this was extended to more general equations of state (Shyue, 1998; Saurel \& Abgrall, 1999b; Shyue, 2001), and to multiphase and reactive flows (Abgrall \& Saurel, 2003; Billet \& Abgrall, 2003). The difficulty resides in maintaining the pressure equilibrium across the interface despite numerical dissipation (Karni, 1994) and in coupling advection equations to the Euler system in a consistent fashion (Abgrall, 1996; Fedkiw et al., 2000). The implementation of non-oscillatory methods, such as the finite difference WENO scheme in Marquina \& Mulet (2003), does not suppress these oscillations.

In analogy to shock capturing, the methods described above are termed interfacecapturing, because the interface is not explicitly tracked but allowed to diffuse numerically. Sharp interfaces can be achieved using interface-tracking methods, where a level set function (Osher \& Sethian, 1988) or marker particles (Tryggvason et al., 2001) track the interface. However, such formulations often involve slight modifications to the governing equations. Karni $(1994,1996)$ replaced the energy equation by a pressure evolution equation near interfaces. In the Ghost Fluid Method (Fedkiw et al., 1999) and simplified versions thereof (Abgrall \& Karni, 2001; Koren et al., 2002), thermodynamically similar variables are added across interfaces to complete stencils. Jenny et al. (1997) provided a correction for the internal energy based on an updated value of the ratio of specific heats. These methods do not generate spurious oscillations at interfaces and can be used with WENO schemes. However, they are not discretely conservative (Abgrall \& Karni, 2001; Liu et al., 2003), making them less desirable for problems where shockwaves are involved. An important drawback of sharp-interface methods for the simulation of compressible multicomponent flows is that ill-posed features may develop (Samtaney \& Pullin, 1996), as described in greater detail in Section 3.8.

\subsubsection{Present methodology}

The present goal is to simulate compressible multicomponent flow problems with a numerical method that satisfies certain important numerical properties mentioned 
in the previous section:

1. High-order accurate: good convergence properties in smooth regions and little dissipation at discontinuities,

2. Discretely conservative: discrete conservation of mass, momentum and energy, so that correct wave speeds are achieved, and

3. Oscillation-free: prevention of spurious oscillations at shockwaves and interfaces.

From a practical standpoint, it is also desirable that the scheme be computationally efficient and easy to implement. In order to achieve these goals, the existing quasiconservative interface-capturing formulation of Abgrall (1996) and Shyue (1998) is extended by implementing a high-order accurate WENO reconstruction of the average primitive variables and modifying the HLLC solver to solve the advection equations.

\subsection{Time-marching scheme}

A total variation diminishing (TVD) Runge-Kutta (RK) scheme (Shu, 1988; Gottlieb \& Shu, 1998) is employed to march the equations forward in time. These explicit schemes are used to solve systems of initial value problems of the type $q_{t}=L(q)$, which result from a method of lines approximation to the partial differential $q_{t}=-f(q)_{x}$. The total variation diminishing property is especially desirable for nonlinear systems like the Euler equations in order to prevent oscillations. In particular, the following third-order accurate scheme (RK3TVD) is used:

$$
\begin{aligned}
q^{(1)} & =q^{n}+\Delta t L\left(q^{n}\right) \\
q^{(2)} & =\frac{3}{4} q^{n}+\frac{1}{4} q^{(1)}+\frac{1}{4} \Delta t L\left(q^{(1)}\right) \\
q^{n+1} & =\frac{1}{3} q^{n}+\frac{2}{3} q^{(2)}+\frac{2}{3} \Delta t L\left(q^{(2)}\right),
\end{aligned}
$$


with a critical CFL coefficient of 1 . The scheme 3.1 has the advantage of requiring few storage locations, which is important for large-scale computations, and few right-hand-side (RHS) evaluations. In comparison to the three RHS evaluations and three storage locations of RK3TVD, six RHS evaluations and five storage locations are required for the corresponding fourth-order accurate RK TVD scheme. Though the order of accuracy of the time-marching scheme is important for smooth problems, results for bubble collapse problems hardly differ when using RK3TVD or RK4TVD.

\subsection{Shock-capturing scheme}

\subsubsection{Finite volume vs. conservative finite difference}

A comparison between finite volume (FV) and conservative finite difference (FD) ENO and WENO approximations is first provided, following Shu (1997). It is shown in Section 3.4.1 that a FV formulation must be used to suppress oscillations generated at interfaces, so this formulation will be described in greater detail in the following sections. In the computational cell $I_{i}=\left[x_{i}-\Delta x / 2, x_{i}+\Delta x / 2\right]$, equation (2.1) can be written in semi-discrete form

$$
\frac{d \tilde{\mathbf{q}}_{i}}{d t}=-\frac{\mathbf{f}_{i+1 / 2}-\mathbf{f}_{i-1 / 2}}{\Delta x}
$$

where $\tilde{\mathbf{q}}_{i}$ approximates the conserved variable, $\mathbf{q}$, at $i$, and $\mathbf{f}_{i+1 / 2}$ approximates the flux, $\mathbf{f}(\mathbf{q})$, at the cell edge, $i+1 / 2$. In the FV formulation, $\tilde{\mathbf{q}}_{i}$ is the cell-average value of $\mathbf{q}$ in $I_{i}$, and is reconstructed on either side of each cell edge, thus yielding a Riemann problem with left and right states, $\mathbf{q}_{i+1 / 2}^{L}$ and $\mathbf{q}_{i+1 / 2}^{R}$. An approximate Riemann solver provides the correct upwind numerical flux. In the FD formulation, $\tilde{\mathbf{q}}_{i}$ is the point value of $\mathbf{q}$ at $x_{i}$, and positive and negative fluxes defined at the cell centers are interpolated at the cell edges. A flux-splitting scheme is used to compute the correct upwind numerical flux. 


\subsubsection{Finite volume WENO reconstruction}

In first-order FV methods, the left and right states of the Riemann problem are reconstructed from the cell averages in a piecewise constant fashion. Such methods can be extended to second-order accuracy by using limiters (LeVeque, 2002). ENO reconstruction (Harten et al., 1987; Shu \& Osher, 1988) is based on adaptive stencils, such that the optimal one is chosen. Given its cell-average values, the function is interpolated on either side of the cell edges. This provides high-order accuracy and essentially non-oscillatory behavior. WENO reconstruction (Liu et al., 1994; Jiang \& Shu, 1996) constitutes an improvement over ENO in that it consists of a convex combination of all the candidate stencils.

The following description of FV WENO is based on Shu (1997). The goal of the WENO reconstruction is the following: given the cell-average values, $\bar{v}_{i}, i=$ $0, \ldots, N$, of a function, $v(x)$, approximate the value of $v(x)$ at $x_{i+1 / 2}$ to order $2 k-1$. This is achieved in two steps: first, $k$ polynomials of degree $k-1$ are reconstructed from the cell-average values of $v$; then, these polynomials are combined to provide a $2 k-1^{\text {th }}$ order non-oscillatory approximation to $v$.

A few useful definitions for an arbitrary function are listed in Table 3.1. Based on these definitions, the primitive function can be related to the cell-average value,

$$
V\left[x_{i-1 / 2}, x_{i+1 / 2}\right]=\frac{V\left(x_{i+1 / 2}\right)-V\left(x_{i-1 / 2}\right)}{x_{i+1 / 2}-x_{i-1 / 2}}=\bar{v}_{i},
$$

so that, for uniform grid spacing, $\bar{v}\left[x_{i}, \ldots, x_{i+j+1}\right]=j V\left[x_{i-1 / 2}, \ldots, x_{i+j-1 / 2}\right]$. In addition,

$$
V\left(x_{i+1 / 2}\right)=\sum_{j=-\infty}^{i} \int_{x_{j-1 / 2}}^{x_{i+1 / 2}} v(\xi) d \xi=\sum_{j=-\infty}^{i} \bar{v}_{j} \Delta x_{j}
$$

\section{Reconstruction from cell-average values}

The goal of the reconstruction is the following: given the cell-average values, $\bar{v}_{i}$, of a function, $v(x)$, find a polynomial, $p(x)$, of degree at most $k-1$ for each cell 
Table 3.1: Definitions of variables used in the derivation of the WENO procedure.

\begin{tabular}{|l|c|}
\hline Cell-average value & $\bar{v}_{i}=\frac{1}{x_{i+1 / 2}-x_{i-1 / 2}} \int_{x_{i-1 / 2}}^{x_{i+1 / 2}} v(\xi) d \xi$ \\
\hline Primitive function & $V(x)=\int_{-\infty}^{x} v(\xi) d \xi$ \\
\hline & $v\left[x_{i+1 / 2}\right]=v\left(x_{i+1 / 2}\right)$ \\
& $v\left[x_{i-1 / 2}, x_{i+1 / 2}\right]=\frac{v\left(x_{i+1 / 2}\right)-v\left(x_{i-1 / 2}\right)}{x_{i+1 / 2}-x_{i-1 / 2}}$ \\
$\ldots$ \\
Divided differences & \\
& $v\left[x_{i-1 / 2}, \ldots, x_{i+j+1 / 2}\right]=\frac{v\left[x_{i+1 / 2}, \ldots, x_{i+j-1 / 2}\right]-v\left[x_{i-1 / 2}, \ldots, x_{i+j-3 / 2}\right]}{x_{i+j+1 / 2}-x_{i-1 / 2}}$ \\
\hline
\end{tabular}

$I_{i}$ such that it is a $k$ th-order accurate approximation of $v(x)$ on $I_{i}$, i.e., $p_{i}(x)=$ $v(x)+O\left(\Delta x^{k}\right), \forall x \in I_{i}$. In particular,

$$
\begin{aligned}
& v_{i+1 / 2}^{L}=p_{i}\left(x_{i+1 / 2}\right)=v\left(x_{i+1 / 2}\right)+O\left(\Delta x^{k}\right), \\
& v_{i-1 / 2}^{R}=p_{i}\left(x_{i-1 / 2}\right)=v\left(x_{i-1 / 2}\right)+O\left(\Delta x^{k}\right) .
\end{aligned}
$$

The subscript $i$ of $p$ denotes that the polynomial is evaluated in cell $I_{i}$. The superscripts $L$ and $R$ of $v_{i \pm 1 / 2}$ denote at which side of the cell edge (left or right) the polynomial is evaluated. ${ }^{2}$

Given the location of $I_{i}$ and the order of accuracy, $k$, a stencil based on $r$ cells to the left and $s$ cells to the right of $I_{i}, S(i)=\left\{I_{i-r}, \ldots, I_{i+s}\right\}$, is defined, where $r+s=k-1$ and $r, s \geq 0$. Then, there exists a unique polynomial of degree at most $k-1=r+s$, denoted by $p(x)$, whose cell average in each of the cells in $S(i)$ agrees with that of $v(x)$. The unique polynomial of degree at most $k$, which interpolates $V(x)$ at the $k+1$ points, $\left\{x_{i-r-1 / 2}, \ldots, x_{i+s+1 / 2}\right\}$, is denoted $P(x)$, and

\footnotetext{
${ }^{2}$ The polynomials in cells $I_{i}$ and $I_{i+1}$ both are evaluated at $x_{i+1 / 2}$, one on the left of the cell edge, one on the right. A Riemann solver then computes the appropriate state at the cell edge.
} 
its derivative $p(x)$. Then, it follows that

$$
\begin{aligned}
& \frac{1}{\Delta x_{j}} \int_{x_{j-1 / 2}}^{x_{j+1 / 2}} p(\xi) d \xi=\frac{1}{\Delta x_{j}} \int_{x_{j-1 / 2}}^{x_{j+1 / 2}} P^{\prime}(\xi) d \xi=\frac{P\left(x_{i+1 / 2}\right)-P\left(x_{i-1 / 2}\right)}{\Delta x_{j}} \\
& \quad=\frac{V\left(x_{i+1 / 2}\right)-V\left(x_{i-1 / 2}\right)}{\Delta x_{j}}=\frac{1}{\Delta x_{j}}\left(\int_{-\infty}^{x_{j+1 / 2}} v(\xi) d \xi-\int_{-\infty}^{x_{j-1 / 2}} v(\xi) d \xi\right)=\bar{v}_{j}
\end{aligned}
$$

where $j=i-r, \ldots, i+s$. The third equality holds true because $P$ interpolates $V$ at the points $x_{j \pm 1 / 2}$ by definition. Uniqueness can be shown by considering two functions, $p_{1}$ and $p_{2}$, whose cell-average values are both equal to that of $v$. Since $P$ approximates $V$ to order $k+1$ in $I_{i}$, then

$$
P^{\prime}(x)=V^{\prime}(x)+O\left(\Delta x^{k}\right) \quad \Rightarrow \quad p(x)=v(x)+O\left(\Delta x^{k}\right), \quad \forall x \in I_{i} .
$$

In order to calculate the reconstructed polynomial $p(x)$, the Newton form of $P$,

$$
P(x)=\sum_{j=0}^{k} V\left[x_{i-r-1 / 2}, \ldots, x_{i-r+j-1 / 2}\right] \prod_{m=0}^{j-1}\left(x-x_{i-r+m-1 / 2}\right),
$$

is differentiated to obtain:

$$
p(x)=\sum_{j=1}^{k} V\left[x_{i-r-1 / 2}, \ldots, x_{i-r+j-1 / 2}\right] \sum_{m=0}^{j-1} \prod_{\substack{l=0 \\ l \neq m}}^{j-1}\left(x-x_{i-r+l-1 / 2}\right) .
$$

Since the mapping from the given cell-average values to the interpolating polynomial is linear, there exist constants, $c_{r j}$, that are functions of $r, k$, and $\Delta x_{j}$ (but not of $v$ ), such that

$$
v_{i+1 / 2}^{L}=p\left(x_{i+1 / 2}\right)=\sum_{j=0}^{k-1} c_{r j} \bar{v}_{i-r+j}=v\left(x_{i+1 / 2}\right)+O\left(\Delta x^{k}\right)
$$

is a $k$ th order approximation to $v\left(x_{i+1 / 2}\right)$. Similarly, $v_{i-1 / 2}^{R}=\sum_{j=0}^{k-1} \tilde{c}_{r j} \bar{v}_{i-r+j}$, where $\tilde{c}_{r j}=c_{r-1, j}$. In order to determine the coefficients $c_{r j}$, the Lagrange form 
of $P$ is more useful:

$$
P(x)=\sum_{m=0}^{k} V\left(x_{i-r+m-1 / 2}\right) \prod_{\substack{l=0 \\ l \neq m}}^{k} \frac{x-x_{i-r+l-1 / 2}}{x_{i-r+m-1 / 2}-x_{i-r+l-1 / 2}} .
$$

Using the identity

$$
1=\sum_{m=0}^{k} \prod_{\substack{l=0 \\ l \neq m}}^{k} \frac{x-x_{i-r+l-1 / 2}}{x_{i-r+m-1 / 2}-x_{i-r+l-1 / 2}}
$$

it follows that

$$
P(x)-V\left(x_{i-r-1 / 2}\right)=\sum_{m=0}^{k}\left(V\left(x_{i-r+m-1 / 2}\right)-V\left(x_{i-r-1 / 2}\right)\right) \prod_{\substack{l=0 \\ l \neq m}}^{k} \frac{x-x_{i-r+l-1 / 2}}{x_{i-r+m-1 / 2}-x_{i-r+l-1 / 2}} .
$$

Futhermore,

$$
V\left(x_{i-r+m-1 / 2}\right)-V\left(x_{i-r-1 / 2}\right)=\sum_{j=0}^{m-1} \bar{v}_{i-r+j} \Delta x_{i-r+j} .
$$

Thus, taking the derivative,

$$
p(x)=\sum_{m=0}^{k} \sum_{j=0}^{m-1} \bar{v}_{i-r+j} \Delta x_{i-r+j} \frac{\sum_{\substack{l=0 \\ l \neq m}}^{k} \prod_{\substack{q=0 \\ q \neq m, l}}^{k}\left(x-x_{i-r+q-1 / 2}\right)}{\prod_{\substack{l=0 \\ l \neq m}}^{k}\left(x_{i-r+m-1 / 2}-x_{i-r+l-1 / 2}\right)} .
$$

It should be noted that the following identity holds true:

$$
\sum_{m=0}^{k} \sum_{j=0}^{m-1} \equiv \sum_{j=0}^{k-1} \sum_{m=j+1}^{k}
$$

Thus,

$$
c_{r j}=\left(x_{i-r+j+1 / 2}-x_{i-r+j-1 / 2}\right) \sum_{m=j+1}^{k} \frac{\sum_{\substack{l=0 \\ l \neq m}}^{k} \prod_{\substack{q=0 \\ q \neq l, m}}^{k}\left(x_{i+1 / 2}-x_{i-r+q-1 / 2}\right)}{\prod_{\substack{l=0 \\ l \neq m}}^{k}\left(x_{i-r+m-1 / 2}-x_{i-r+l-1 / 2}\right)} .
$$




\section{WENO reconstruction}

The $k$ candidate stencils, $S_{r}(i)=\left\{x_{i-r}, \ldots, x_{i-r+k-1}\right\}$, produce $k$ different reconstructions to $v\left(x_{i+1 / 2}\right)$ :

$$
p_{i+1 / 2}^{(r)}=\sum_{j=0}^{k-1} c_{r j} \bar{v}_{i-r+j}=v\left(x_{i+1 / 2}\right)+O\left(\Delta x^{k}\right) .
$$

In order to obtain a non-oscillatory $2 k$-1th-order accurate approximation of $v\left(x_{i+1 / 2}\right)$, the $k$ polynomials on $S_{r}(i)$ are combined:

$$
v_{i+1 / 2}=\sum_{r=0}^{k-1} \omega_{r} p_{i+1 / 2}^{(r)}+O\left(\Delta x^{2 k-1}\right)
$$

where $\omega_{r}$ are nonlinear weights. If $v(x)$ is smooth in all candidate stencils, then there are constants, $d_{r}$, such that a convex combination of the polynomials is taken:

$$
v_{i+1 / 2}=\sum_{r=0}^{k-1} d_{r} p_{i+1 / 2}^{(r)}+O\left(\Delta x^{2 k-1}\right)
$$

where $\sum_{r=0}^{k-1} d_{r}=1, d_{r} \geq 0$. The weights $\omega_{r}$, are constructed based on $d_{r}$. For consistency, it is also required that $\sum_{r=0}^{k-1} \omega_{r}=1$ and $\omega_{r} \geq 0$. In order to satisfy the accuracy requirement of Equation 3.19, $\omega_{r}=d_{r}+O\left(\Delta x^{k-1}\right)$. This follows from

$$
\begin{gathered}
\sum_{r=0}^{k-1} \omega_{r} p_{i+1 / 2}^{(r)}-\sum_{r=0}^{k-1} d_{r} p_{i+1 / 2}^{(r)}=\sum_{r=0}^{k-1}\left(\omega_{r}-d_{r}\right) p_{i+1 / 2}^{(r)}-\left(\sum_{r=0}^{k-1}\left(\omega_{r}-d_{r}\right)\right) v_{i+1 / 2} \\
=\sum_{r=0}^{k-1}\left(\omega_{r}-d_{r}\right)\left(p_{i+1 / 2}^{(r)}-v_{i+1 / 2}\right)=\sum_{r=0}^{k-1} O\left(\Delta x^{k-1}\right) O\left(\Delta x^{k}\right)=O\left(\Delta x^{2 k-1}\right) .
\end{gathered}
$$

The weights can then be defined as

$$
\omega_{r}=\frac{\alpha_{r}}{\sum_{s=0}^{k-1} \alpha_{s}}, \quad \alpha_{r}=\frac{d_{r}}{\left(\epsilon+\beta_{r}\right)^{2}},
$$


where $\epsilon$ is a small parameter that prevents division by zero and the smoothness indicators, $\beta_{r}$, are the sum of the squares of the scaled $L_{2}$ norms for all derivatives of the interpolation polynomial over the interval $\left(x_{i-1 / 2}, x_{i+1 / 2}\right)$ :

$$
\beta_{r}=\sum_{l=1}^{k-1} \int_{x_{i-1 / 2}}^{x_{i+1 / 2}} \Delta x^{2 l-1} \frac{d^{l} p^{(r)}(x)}{d x^{l}} d x .
$$

If the solution is smooth, $\beta_{r}=O\left(\Delta x^{2}\right)$, while $\beta_{r}=O(1)$ when discontinuities are present. In practice, $\epsilon \approx 10^{-5}-10^{-7}$ (Jiang \& Shu, 1996).

\section{Further WENO extensions}

Further improvements to the WENO methodology can be implemented. For instance, artificial compression (Yang, 1990) may be used to obtain sharper contact discontinuities. Tools to achieve higher-order accuracy include monotonicitypreserving WENO schemes (Balsara \& Shu, 2000), which preserve the monotonicity of the reconstructed function, and Mapped WENO schemes (Henrick et al., 2005), which provide more accurate weights.

Multidimensional ENO and WENO schemes can easily be implemented in the FD formulation dimension by dimension (Shu, 1999). On the other hand, a two-dimensional FV reconstruction is computationally intensive, because two one-dimensional reconstructions are needed per grid point. Since the fluxes must be averaged along the cell edges, a Gaussian quadrature rule is used in multiple dimensions (Grasso \& Pirozzoli, 2000; Titarev \& Toro, 2004). After the WENO reconstruction, $\mathbf{q}_{i+1 / 2, j}^{L}$ and $\mathbf{q}_{i+1 / 2, j}^{R}$ are reconstructed in the transverse direction, thus yielding the values at $j \pm 1 / 2 \sqrt{3}$ required by the Gaussian quadrature.

The reconstruction is often performed in characteristic space because of the selfsimilar nature of the Riemann problem for the Euler equations (2.1). In nonlinear systems of equations, oscillations can develop in component-wise reconstruction due to the interaction of discontinuities of different characteristic fields, regardless of the CFL constraint (Harten et al., 1987; Qiu \& Shu, 2002). Thus, a reconstruction in characteristic space is preferred. The variables are first locally decomposed 
onto the respective characteristic fields and then reconstructed; thereafter, they are projected back into physical space (Shu, 1999). The advection equations are already in characteristic form, so that the projection onto characteristic space is trivial. Although this reconstruction is more expensive computationally, each field is treated separately, thus avoiding collisions between characteristics.

The extensions to WENO discussed in this section have been implemented in the present chapter for a uniform grid. However, for bubble collapse problems, a non-uniform grid is used in order to provide high resolution near the bubble and to prevent reflections from the boundaries to affect the solution, as described in Section 3.7.3. The derivation of the polynomials, ideal weights, and smoothness indicators for a non-uniform grid is provided in Appendix B.

\subsubsection{The HLLC approximate Riemann solver}

Because the Riemann problem resulting from the reconstruction is computationally expensive to solve exactly, an approximate Riemann solver is used. Examples thereof include the Roe (Roe, 1981) and HLL (Harten et al., 1983) solvers; the Lax-Friedrichs solver is a special case of the HLL solver (Toro, 1999). Roe solvers are less dissipative, but more computationally intensive. More importantly, they do not preserve positivity (Einfeldt et al., 1991). This is a critical property when computing problems where low densities and pressures are achieved; this situation can occur in a number of compressible multicomponent flow calculations. Thus, HLL solvers are favored, in particular the HLLC solver (Toro et al., 1994; Toro, 1999), because it resolves discontinuities more sharply, and isolated shockwaves and contacts exactly (Batten et al., 1997). Given allowable left and right states, the HLLC solver preserves positivity (Batten et al., 1997).

The HLLC solver is an extension to the HLL solver, whereby the contact discontinuity is restored. The HLLC flux can be written:

$$
\mathbf{f}^{H L L C}=\frac{1+\operatorname{sign}\left(s^{*}\right)}{2}\left[\mathbf{f}^{L}+s^{-}\left(\mathbf{q}^{* L}-\mathbf{q}^{L}\right)\right]+\frac{1-\operatorname{sign}\left(s^{*}\right)}{2}\left[\mathbf{f}^{R}+s^{+}\left(\mathbf{q}^{* R}-\mathbf{q}^{R}\right)\right],
$$


where the intermediate "star" state is defined as

$$
\mathbf{q}^{* k}=\chi^{k}\left(\begin{array}{c}
\rho^{k} \\
\rho^{k} s^{*} \\
E^{k}+\left(s^{*}-u^{k}\right)\left(\rho^{k} s^{*}+\frac{p^{k}}{s^{k}-u^{k}}\right)
\end{array}\right), \quad \chi^{k}=\frac{s^{k}-u^{k}}{s^{k}-s^{*}}
$$

where $k=L, R$. Following Einfeldt et al. (1991), the wave speeds are given by

$$
s^{-}=\min \left(0, s^{L}\right), \quad s^{+}=\max \left(0, s^{R}\right)
$$

where

$$
s^{L}=\left((u-c)^{R O E}, u^{L}-c^{L}\right), \quad s^{R}=\left((u+c)^{R O E}, u^{R}+c^{R}\right) .
$$

The intermediate wave speed is computed according to Batten et al. (1997):

$$
s^{*}=\frac{p^{R}-p^{L}+\rho^{L} u^{L}\left(s^{L}-u^{L}\right)-\rho^{R} u^{R}\left(s^{R}-u^{R}\right)}{\rho^{L}\left(s^{L}-u^{L}\right)-\rho^{R}\left(s^{R}-u^{R}\right)} .
$$

\section{Adapting the HLLC solver to the advection equation}

Although hyperbolic, advection equations are not conservation laws, so it is not immediately clear how to implement any approximate Riemann solver consistently with the Euler equations. The Lax-Friedrichs, Roe, and HLL solvers have been adapted to the advection equation (Abgrall, 1996; Shyue, 1998; Saurel \& Abgrall, 1999b). In order to adapt the HLLC solver, it is tempting follow the same thought process by considering two states connected by a wave moving at speed, $u_{i}$. Equation 2.8 becomes, in semi-discrete form:

$$
\frac{d \bar{\phi}_{i}}{d t}=-\frac{u_{i}}{\Delta x}\left[\frac{1+\operatorname{sign}\left(u_{i}\right)}{2}\left(\phi_{i+1 / 2}^{L}-\phi_{i-1 / 2}^{L}\right)+\frac{1-\operatorname{sign}\left(u_{i}\right)}{2}\left(\phi_{i+1 / 2}^{R}-\phi_{i-1 / 2}^{R}\right)\right] .
$$

It is easy to verify that this leads to the oscillation-free advection of an isolated interface and propagation of isolated shocks. However, if the pressure varies strongly for non-constant $\phi$, the wavespeed in the advection equation, $u_{i}$, is inconsistent 
with that of the contact discontinuity, $s_{i \pm 1 / 2}^{*}$, in the Euler equations. Equation 2.8 can be rewritten using the chain rule as

$$
\phi_{t}+\mathbf{g}_{x}-\phi u_{x}=0, \quad \mathbf{g}=u \phi
$$

The second term is a conservative flux, and the velocity differentiation in the third term allows the HLLC solver to be adapted. Integrating the advection equation 3.30 over a cell,

$$
\left.\frac{d \overline{\boldsymbol{\phi}}}{d t}\right|_{i}=-\frac{1}{\Delta x}\left(\mathbf{g}_{i+1 / 2}-\mathbf{g}_{i-1 / 2}\right)+\frac{1}{\Delta x} \int_{x_{i}-1 / 2}^{x_{i}+1 / 2} \phi \frac{\partial u}{\partial x} d x
$$

This equation is exact. The following approximation is made to evaluate the integral:

$$
\left.\frac{d \overline{\boldsymbol{\phi}}}{d t}\right|_{i}=-\frac{1}{\Delta x}\left(\mathbf{g}_{i+1 / 2}-\mathbf{g}_{i-1 / 2}\right)+\frac{1}{\Delta x} \bar{\phi}_{i}\left(u_{i+1 / 2}-u_{i-1 / 2}\right) .
$$

Equation 3.32 is exact far from interfaces, where $\phi$ is assumed constant. At discontinuities, shock-capturing schemes can achieve no better than first-order accuracy (LeVeque, 2002); since the integral in Equation 3.31 is evaluated using a midpoint rule and the derivative using a centered scheme, it is at worst second-order accurate. Therefore, this approximation does not deteriorate the overall order of accuracy of the method. Based on the conservative form of the advection equation (Toro, 1999), the velocity in the source term is computed as

$$
u^{H L L C}=\frac{1+\operatorname{sign}\left(s^{*}\right)}{2}\left[u^{L}+s^{-}\left(\chi^{* L}-1\right)\right]+\frac{1-\operatorname{sign}\left(s^{*}\right)}{2}\left[u^{R}+s^{+}\left(\chi^{* R}-1\right)\right] .
$$




\subsection{Interface-capturing scheme}

\subsubsection{Occurrence of oscillations at isolated interfaces}

Since the present scheme is shock-capturing, isolated shockwaves and rarefaction waves are treated appropriately. Across isolated material interfaces, the model equations $(2.1,2.8)$ preserve the pressure equilibrium (Shyue, 1998). The goal is to provide a spatial discretization scheme that satisfies this condition.

To motivate the numerical method, the analysis of Abgrall (1996), Abgrall \& Karni (2001) and Shyue (1998) is followed, where the problem of the advection of an isolated interface between two different gases at constant velocity is considered. The kinematic and dynamic interface conditions require $u$ and $p$ to be uniform in time and space; therefore, the Euler equations 2.1 become:

$$
\begin{aligned}
\frac{\partial \rho}{\partial t} & =-u \frac{\partial \rho}{\partial x} \\
\frac{\partial(\rho u)}{\partial t} & =-u \frac{\partial \rho}{\partial x} \\
\frac{\partial E}{\partial t} & =-u\left(\frac{u^{2}}{2} \frac{\partial \rho}{\partial x}+p \frac{\partial \Gamma}{\partial x}\right) .
\end{aligned}
$$

Combining Equations 3.34a and 3.34b, $d u / d t=0$. Equation 3.34c and the equation of state 2.6 yield $d p / d t=0$ if

$$
\frac{\partial \Gamma}{\partial t}=-u \frac{\partial \Gamma}{\partial x}
$$

In FV methods, the conservative variables, $\mathbf{q}=(\rho, \rho u, E, \rho \Gamma)^{T}$, are reconstructed. However, the dissipation introduced in these variables, in particular in $E$ and $\rho \Gamma$, does not maintain pressure equilibrium (Karni, 1994; Saurel \& Abgrall, 1999b), especially when using a nonlinear reconstruction such as WENO. For the same reason, FD methods fail.

In order to preserve the pressure equilibrium in the discrete equations, the behavior of the continuous equations 3.34 and 3.35 is emulated by reconstructing $u$ and $p$ individually. Since the elements of the interpolating stencils are identical near isolated interfaces, the equilibrium in these variables is preserved. Thus, the 
primitive variables, $\mathbf{u}=(\rho, u, p, \Gamma)^{T}$, must be reconstructed, rather than $\mathbf{q}$. This allows the kinematic and dynamic interface conditions to be preserved.

This has further implications for the advection equation. First, the specific function, $\Gamma(\gamma)=1 /(\gamma-1)$, must be advected, not just any arbitrary function of $\gamma$ (Shyue, 1998). Thus, model equations 2.1 and 2.8 must be used. Also, the advection equation must be discretized in a fashion consistent with the energy equation (Abgrall, 1996; Saurel \& Abgrall, 1999b). Coupling a level set equation as in Fedkiw et al. (2000) does not necessarily guarantee this requirement and might thus cause oscillations. It is straightforward to treat the case of $\Pi_{\infty}$ in an analogous fashion (Shyue, 1998).

This analysis shows that, if the above conditions are not met, a discontinuity in $\Gamma$ causes an error in pressure at the interface, which generates oscillations that propagate within the flow. Methods where there is no explicit discontinuity in $\Gamma$

(e.g., Ghost Fluid methods (Fedkiw et al., 1999; Abgrall \& Karni, 2001)) or where $\Gamma$ does not enter the equations at interfaces (e.g., Pressure Evolution methods (Karni, 1994, 1996)) are not affected by this problem.

\subsubsection{Reconstruction of the primitive variables}

At the beginning of each time step, the cell averages of the conservative quantities are given. In order to reconstruct the primitive variables, the local average velocity, $\bar{v}$, and pressure, $\bar{p}$, are defined:

$$
\bar{v}_{i} \triangleq \frac{\overline{\rho u}_{i}}{\bar{\rho}_{i}}, \quad \bar{p}_{i} \triangleq \frac{\bar{E}_{i}-\bar{\rho}_{i} \frac{\bar{v}_{i}^{2}}{2}}{\bar{\Gamma}_{i}}
$$

The average velocity, $\bar{v}$, is analogous to the density-weighted average used in compressible turbulence models (Favre, 1965). These variables, along with $\bar{\rho}_{i}$ and $\bar{\Gamma}_{i}$, are reconstructed using WENO. This provides the left and right states for the Riemann problem at the cell edges.

To understand the impact of this methodology on the accuracy of the scheme, 
the sliding average of a function, $q(x)$, is defined by

$$
\bar{q}(x) \triangleq \frac{1}{\Delta x} \int_{x-\Delta x / 2}^{x+\Delta x / 2} q(\xi) d \xi, \quad \bar{q}_{i}=\bar{q}\left(x_{i}\right)
$$

so that in smooth regions, $\bar{q}(x)=q(x)+O\left(\Delta x^{2}\right)$. ENO and WENO reconstructions have the following property (Harten et al., 1987; Shu, 1999):

$$
\overline{\tilde{q}}\left(x_{i}\right)=\bar{q}_{i},
$$

where $\tilde{q}(x)$ is a pointwise approximation to $q(x)$. Therefore, the reconstruction preserves the total amount of $q$ in each cell. Equation 3.37 implies that $\bar{u}(x)=u(x)+O\left(\Delta x^{2}\right)$. Using Taylor series expansion, $\bar{v}(x)=u(x)+O\left(\Delta x^{2}\right)$, so that $\bar{v}$ approximates $u(x)$ to the same order that $\bar{u}$ does. This justifies the definitions in Equation 3.36, which are the building blocks of the reconstruction. From Equation 3.38, given the reconstructed density, $\tilde{\rho}(x)$, and momentum, $\widetilde{\rho u}(x)$, the reconstructed velocity, $\tilde{v}(x)$, satisfies

$$
\overline{\tilde{\rho}}(x) \overline{\tilde{v}}(x)=\overline{\widetilde{\rho u}}(x)
$$

In other words, $\tilde{v}(x)$ is a high-order accurate approximation to the velocity, which preserves the total momentum in each cell. Therefore, $\rho u_{i+1 / 2}=\rho_{i+1 / 2} v_{i+1 / 2}$ to high-order accuracy. Similarly, $\tilde{p}(x)$ is a high-order accurate approximation to the pressure, which preserves the total energy in each cell. The convergence analysis presented in Section 3.6.1 indicates that the proper convergence rate is achieved. Characteristic reconstruction can easily be implemented, as the characteristic variables are naturally expressed in terms of primitive variables. Furthermore, the Euler equations 2.1 are still written in flux difference form (LeVeque, 2002):

$$
\mathbf{f}_{i+1 / 2}=\mathbf{f}_{(i+1)-1 / 2}
$$

Equations 3.39 and 3.40 show that the numerical scheme is discretely conservative 
(LeVeque, 2002) for the Euler equations.

\subsubsection{Oscillation-free advection of an isolated interface}

The present scheme can be shown to maintain pressure equilibrium for the problem of the advection of an isolated interface between two gases at constant speed, $u$. Without loss of generality, the case $\left|s^{k}\right|>u>0$ is considered, so that $\mathbf{q}^{* L}=\mathbf{q}^{L}$ and $u^{* L}=u^{L}=u$. From the interface conditions, the velocity, $u$, and the pressure, $p$, are uniform. Then, using the HLLC solver 3.24 and 3.33 and the reconstruction of the primitive variables described above, the Euler and advection equations 2.1 and 2.8 can be marched forward by a time step:

$$
\begin{aligned}
\bar{\rho}_{i}^{n+1} & =\bar{\rho}^{n}-\frac{\Delta t}{\Delta x} u\left(\rho_{i+1 / 2}^{L}-\rho_{i-1 / 2}^{L}\right) \\
\overline{\rho u}_{i}^{n+1} & =\overline{\rho u}_{i}^{n}-\frac{\Delta t}{\Delta x} u^{2}\left(\rho_{i+1 / 2}^{L}-\rho_{i-1 / 2}^{L}\right) \\
\bar{E}_{i}^{n+1} & =\bar{E}_{i}^{n}-\frac{\Delta t}{\Delta x} u\left[\frac{u^{2}}{2}\left(\rho_{i+1 / 2}^{L}-\rho_{i-1 / 2}^{L}\right)+p\left(\Gamma_{i+1 / 2}^{L}-\Gamma_{i-1 / 2}^{L}\right)\right] \\
\bar{\Gamma}_{i}^{n+1} & =\bar{\Gamma}_{i}^{n}-\frac{\Delta t}{\Delta x} u\left(\Gamma_{i+1 / 2}^{L}-\Gamma_{i-1 / 2}^{L}\right) .
\end{aligned}
$$

Since $u$ is constant, $\bar{\rho} \bar{u}_{i}^{n}=\bar{\rho}_{i}^{n} u$. The equation of state (2.6) and equations (3.41) can be combined to yield the velocity and pressure at the next time step:

$$
u_{i}^{n+1} \triangleq \frac{\overline{\rho u}_{i}^{n+1}}{\bar{\rho}_{i}^{n+1}}=u, \quad p_{i}^{n+1} \triangleq \frac{\bar{E}_{i}^{n+1}-\frac{\left(\overline{\rho u}_{i}^{n+1}\right)^{2}}{2 \bar{\rho}_{i}^{n+1}}}{\bar{\Gamma}_{i}^{n+1}}=p .
$$

Therefore, the present scheme maintains equilibrium in velocity and pressure for the advection of an isolated interface. This can be generalized to fluids with $P_{\infty} \neq 0$ and to multiple dimensions. However, this derivation cannot readily be extended to problems where interfaces interact with shockwaves; nevertheless, it is demonstrated using difficult test cases that no oscillations are observed. 


\subsection{Boundary conditions}

Two types of boundary conditions are implemented: reflecting boundary conditions, which represent a solid wall, and non-reflecting boundary conditions, which reduce an infinite physical domain to a computational domain of finite size.

\subsubsection{Reflecting boundary conditions}

Reflecting boundary conditions are used to represent a solid boundary in inviscid flow and are constructed such that the no-through-flow condition is satisfied. A centerline approach is used, where scalar quantities (and the tangential velocity component) are mirrored across the wall, while the normal velocity component is reflected; thus, the wall is considered as an axis of symmetry. ${ }^{3}$ In Cartesian rectangular coordinates, the situation is shown in Figure 3.1 for the first point next to the wall. If larger stencils are required, the same procedure is used (e.g., for point $N+2$, the values at $N-2$ are used).

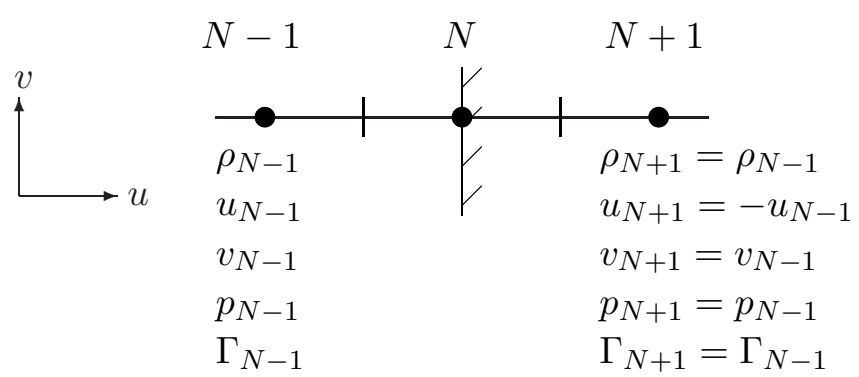

Figure 3.1: Schematic of reflecting boundary conditions on a Cartesian grid.

In FV schemes, both the reconstruction and the flux calculation are affected by this procedure. In the reconstruction, the WENO scheme does not need to be modified at the wall: extra points are added at $N+1, N+2, \ldots$, with the value specified as shown above. Therefore, the reconstruction retains its formal order of accuracy throughout the domain. The reflected fluxes across the wall are then

\footnotetext{
${ }^{3}$ In viscous flows, an additional condition is required for the tangential velocity component.
} 
computed as follows:

$$
\begin{aligned}
\Gamma_{N+1 / 2} & =\Gamma_{N-1 / 2}, \quad \rho u_{N+1 / 2}=-\rho u_{N-1 / 2}, \quad \rho u v_{N+1 / 2}=-\rho u v_{N-1 / 2}, \\
\left(\rho u^{2}+p\right)_{N+1 / 2}=\left(\rho u^{2}+p\right)_{N-1 / 2}, \quad(u(E+p))_{N+1 / 2} & =-(u(E+p))_{N-1 / 2} .
\end{aligned}
$$

\subsubsection{Non-reflecting boundary conditions}

Non-reflecting boundary conditions are constructed such that waves radiate outwards and that no incoming waves enter the domain, as described in Thompson $(1987,1990)$. The theory is based on the characteristic form of the Euler equations 2.3 , which can be written,

$$
\begin{aligned}
& \left(\frac{\partial p}{\partial t}+\rho c \frac{\partial v}{\partial t}\right)+(v+c)\left(\frac{\partial p}{\partial r}+\rho c \frac{\partial v}{\partial r}\right)+\frac{\rho c^{2} v}{r}=0, \\
& \left(\frac{\partial p}{\partial t}-c^{2} \frac{\partial \rho}{\partial t}\right)+v\left(\frac{\partial p}{\partial r}-c^{2} \frac{\partial \rho}{\partial r}\right)=0, \\
& \left(\frac{\partial p}{\partial t}-\rho c \frac{\partial v}{\partial t}\right)+(v-c)\left(\frac{\partial p}{\partial r}-\rho c \frac{\partial v}{\partial r}\right)+\frac{\rho c^{2} v}{r}=0 .
\end{aligned}
$$

In Cartesian rectangular coordinates, the geometrical source terms in 3.44a and $3.44 \mathrm{c}$ do not appear. The eigenvalues are $\lambda_{1}=v-c, \lambda_{2}=v$, and $\lambda_{3}=v+c$. The following variables are then defined:

$\mathcal{L}_{1}=(v-c)\left(\frac{\partial p}{\partial r}-\rho c \frac{\partial v}{\partial r}\right), \quad \mathcal{L}_{2}=v\left(\frac{\partial p}{\partial r}-c^{2} \frac{\partial \rho}{\partial r}\right), \quad \mathcal{L}_{3}=(v+c)\left(\frac{\partial p}{\partial r}+\rho c \frac{\partial v}{\partial r}\right)$

These variables represent the characteristic left- or right-moving waves. In order to satisfy non-reflecting conditions for subsonic outflow, only outgoing waves are

allowed at the edges of the computational domain. This is done by computing the appropriate values for $\mathcal{L}$, as described in Table 3.2. The primitive equations can then be written in terms of $\mathcal{L}_{i}$ :

$$
\frac{d p}{d t}=-\frac{\mathcal{L}_{3}+\mathcal{L}_{1}}{2}-\frac{\rho c^{2} v}{r}, \quad \frac{d v}{d t}=-\frac{\mathcal{L}_{3}-\mathcal{L}_{1}}{2 \rho c}, \quad \frac{d \rho}{d t}=\frac{1}{c^{2}}\left(\frac{d p}{d t}+\mathcal{L}_{2}\right) .
$$


Table 3.2: Computation of characteristic variables at the boundaries.

\begin{tabular}{l|cc} 
Sign of eigenvalue & Left boundary & Right boundary \\
\hline$u-c \geq 0$ & $\mathcal{L}_{1}=0$ & compute $\mathcal{L}_{1}$ \\
$u-c \leq 0$ & compute $\mathcal{L}_{1}$ & $\mathcal{L}_{1}=0$ \\
$u \geq 0$ & $\mathcal{L}_{2}=0$ & compute $\mathcal{L}_{2}$ \\
$u \leq 0$ & compute $\mathcal{L}_{2}$ & $\mathcal{L}_{2}=0$ \\
$u+c \geq 0$ & $\mathcal{L}_{3}=0$ & compute $\mathcal{L}_{3}$ \\
$u+c \leq 0$ & compute $\mathcal{L}_{3}$ & $\mathcal{L}_{3}=0$
\end{tabular}

The main practical issue with non-reflecting boundary conditions is that the spatial discretization scheme must be modified near the boundaries, since the variables at points beyond the boundary are unknown. As a result, biased differencing must be implemented. In the present case, a FV WENO5 scheme is used for the interior points, so that the stencil consists of five points centered about $i$. Thus, for both the second cell and the penultimate cell, one point is missing in the stencil, i.e., an extra point would be required beyond the domain. For these cells, the scheme is modified to a third-order accurate WENO scheme, which uses only a three-point stencil. The first and last points are computed by differentiating the variables using a fourth-order accurate one-sided difference scheme:

$$
\left.\frac{d f}{d x}\right|_{0}=\frac{-25 f_{0}+48 f_{1}-36 f_{2}+16 f_{3}-3 f_{4}}{12 \Delta x}
$$

Thus, the right-hand side is evaluated by computing the (discrete) derivatives of average velocity and pressure (and cell-average $\rho$ and $\Gamma$ ); no flux calculations are required. It should be noted that the equations are not conservative at these points, but this is not relevant, because the shocks are weak by the time they reach the boundaries and the domain is large enough that reflections at the boundaries do not contaminate the solution. 
This type of non-reflecting boundary conditions is not exact in multiple dimensions, so that small amplitude waves may be reflected into the domain. However, as described in Section 3.7.3, the domain is chosen to be large enough, and the mesh stretching allows the waves to dissipate more as they propagate farther away.

\subsection{Verification}

In the present section, a number of problems have been chosen to confirm that the method solves the equations accurately (verification stage). Validation of the scheme is provided throughout Chapter 4 by comparisons with theory and experiments.

In certain test cases, the present method is compared to a standard implementation of finite difference (FD) and finite volume (FV) WENO schemes. The differences lie in the model equations used and the variables that are interpolated or reconstructed using WENO, as listed in Table 3.3. The FD and fully-conservative schemes are implemented using existing methods (Shu, 1997) and are expected to behave in a similar fashion, since the model equations are identical. The quasiconservative FV scheme is implemented following Saurel \& Abgrall (1999b) and is expected to generate smaller oscillations than the former schemes. The present scheme is not expected to generate oscillations. The time-marching is RK3TVD, unless otherwise specified. WENOr denotes a WENO scheme of order $r$. The grid and time stepping are uniform and $\Delta t / \Delta x=0.2$.

\subsubsection{Convergence analysis}

In order to assess the convergence of the present method, a smooth acoustics problem in a single gas component is considered. The primitive variables are perturbed about a steady state,

$$
\rho(x, t)=\rho_{o}+\epsilon \rho^{\prime}(x, t), \quad u(x, t)=\epsilon u^{\prime}(x, t), \quad p(x, t)=p_{o}+\epsilon p^{\prime}(x, t),
$$


Table 3.3: Comparison between the different FD and FV WENO schemes, listing the model equations and variables that are interpolated/reconstructed using WENO.

\begin{tabular}{l|cc} 
Scheme & Model & WENO variables \\
\hline FD & $(2.1,2.9)$ & $\left(\rho u, \rho u^{2}+p,(E+p) u, \rho u \Gamma\right)^{T}$ \\
Fully conservative FV & $(2.1,2.9)$ & $(\rho, \rho u, E, \rho \Gamma)^{T}$ \\
Quasi-conservative FV & $(2.1,2.8)$ & $(\rho, \rho u, E, \Gamma)^{T}$ \\
Present & $(2.1,2.8)$ & $(\rho, u, p, \Gamma)^{T}$
\end{tabular}

where $\epsilon$ is small. Using conservation of mass and momentum, and the definition of the sound speed (Equation A.7), it can be shown that $\rho, u$, and $p$ satisfy the second order wave equation, with wave speed $c_{o}$, to first order in $\epsilon$. The initial perturbation is given by $f(x)=\sin ^{8}(\pi x)$, such that the initial conditions are

$$
\rho(x, 0)=1+\epsilon f(x), \quad u(x, 0)=0, \quad p(x, 0)=\frac{1}{\gamma}+\epsilon f(x) .
$$

As $\epsilon$ increases, the wave steepens and ultimately breaks, so that a shockwave forms. When $\epsilon \approx 0.4$, the wave breaks within a period. Figures 3.2 and 3.3 compare the $L_{1}$ and $L_{\infty}$ errors in density between the present and quasi-conservative FV schemes for $\epsilon=10^{-4}$ and $\epsilon=0.1$, respectively, after one period. The reference solution is the solution computed with a much larger number of points $\left(N_{\text {exact }}=1280\right)$ than those studied $(N=10,20,40,80,160)$. WENO3 and WENO5 are implemented with the HLLC solver and without characteristic reconstruction, though there is hardly any difference if different solvers or characteristic reconstruction are used (Shu, 1999). The time-marching is handled using RK5.

The agreement between the two methods is excellent for $\epsilon=10^{-4}$. A slight departure from the -5 slope near $N=160$ is observed in both methods, as the "exact" solution is approached. The solution is still smooth for $\epsilon=0.1$, although the wave starts to steepen. As anticipated, the convergence rate departs from the expected slope, especially in the $L_{\infty}$ plot. The present method performs slightly 

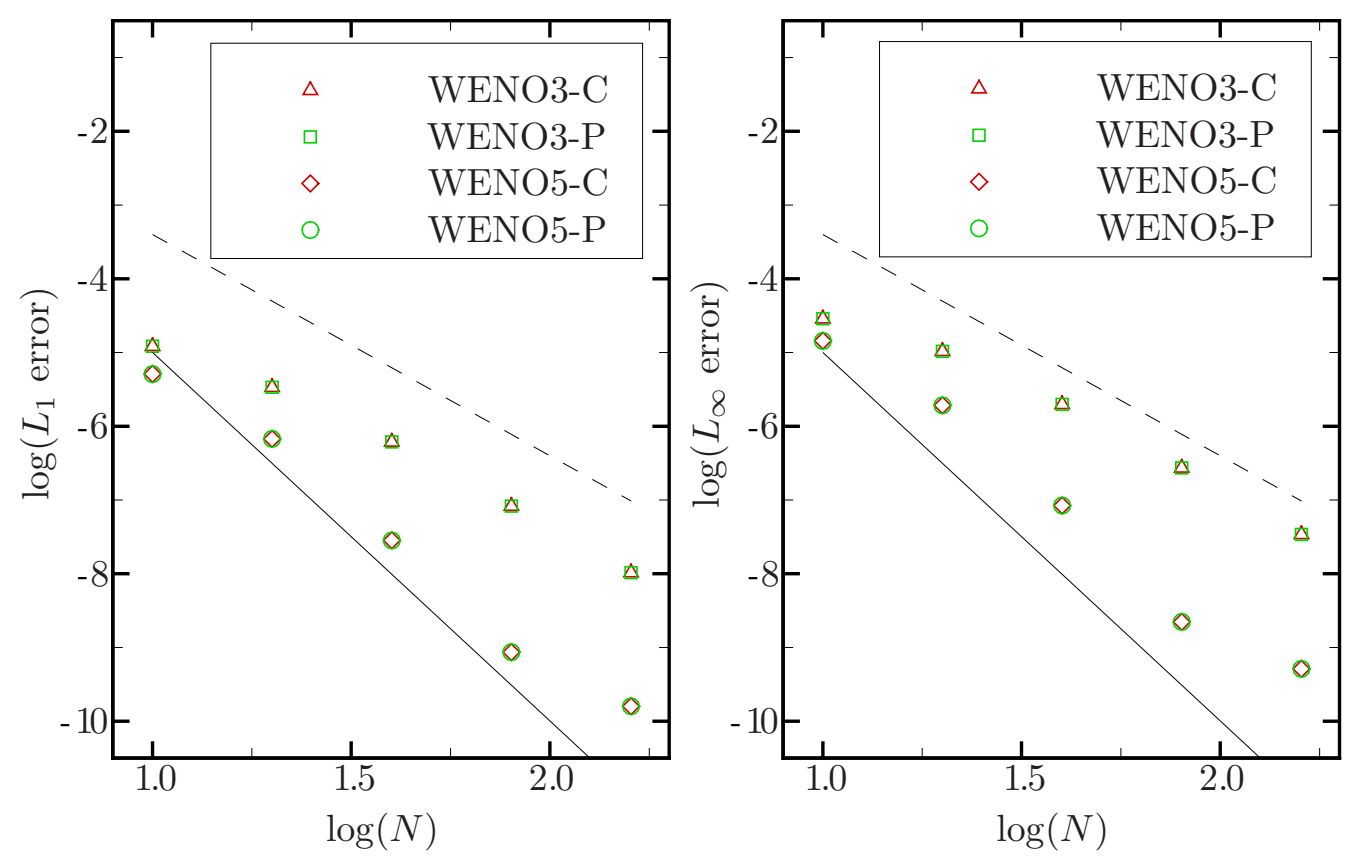

Figure 3.2: $L_{1}$ and $L_{\infty}$ errors in density for a smooth acoustics problem with $\epsilon=10^{-4}$ using WENO3 and WENO5 (C: quasi-conservative scheme; P: present scheme); dashed and solid lines have slopes -3 and -5 , respectively.

better than the quasi-conservative FV scheme when using WENO3, but slightly worse when using WENO5. These discrepancies indicate different sensitivity to the steepening of the waves. However, this feature is not very relevant in practical multi-dimensional computations because the discrepancy is small and the resolution is rarely better than the highest $(N=160)$ shown here.

\subsubsection{Isolated interface advection problem}

The occurrence of oscillations is illustrated for the simplest possible test case, namely the advection of a gas-gas interface, with the following initial states:

$$
(\rho, u, p, \gamma)_{L}^{T}=(1,0.5,1 / 1.4,1.4)^{T}, \quad(\rho, u, p, \gamma)_{R}^{T}=(10,0.5,1 / 1.4,1.6)^{T}
$$

The domain is periodic with $N=100$. The Lax-Friedrichs solver and the cor- 

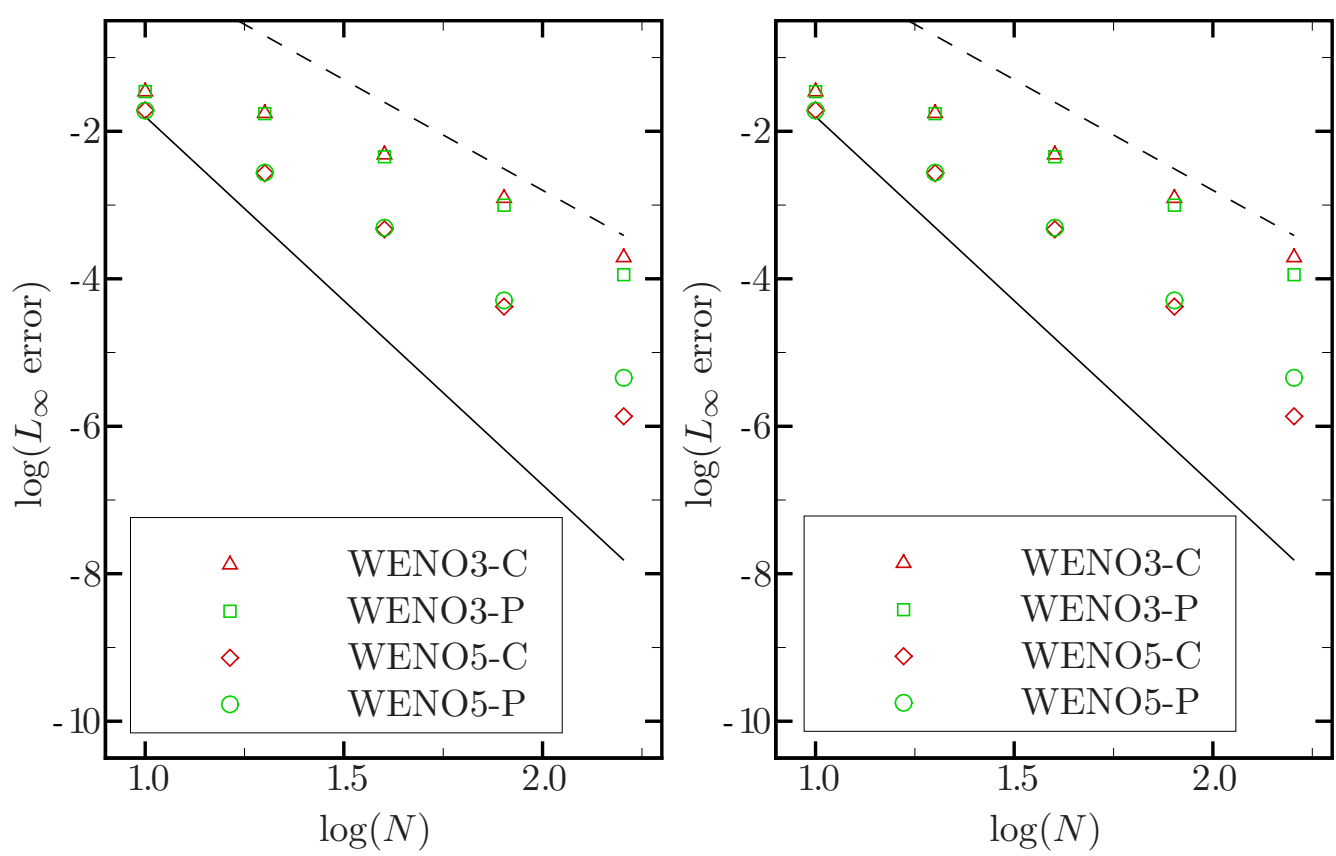

Figure 3.3: $L_{1}$ and $L_{\infty}$ errors in density for a smooth acoustics problem with $\epsilon=10^{-1}$ using WENO3 and WENO5 (C: quasi-conservative scheme; P: present scheme); dashed and solid lines have slopes -3 and -5 , respectively.

responding flux-splitting (Shu, 1999) are implemented in an effort to compare all four schemes of Table 3.3. Figure 3.4 shows density, pressure, $\gamma$, and velocity profiles, as well as the residual mass, momentum, and energy, after subtraction of the total values, after the interface has moved the length of the domain, for the finite difference (FD), fully conservative finite volume (FV-F), and quasi-conservative finite volume (FV-C) cases.

Even though the difference in $\gamma$ is small, oscillations develop at the interface at the first time step and propagate in the flow field thereafter. The FD and fully conservative schemes behave in a similar fashion and generate larger oscillations than the quasi-conservative FV scheme. Nevertheless, the interface is advected at approximately the correct speed despite small errors in velocity due to the oscillations. Although not shown here, the magnitude of the oscillations decreases as the mesh is refined (Abgrall \& Karni, 2001). On the other hand, oscillations 

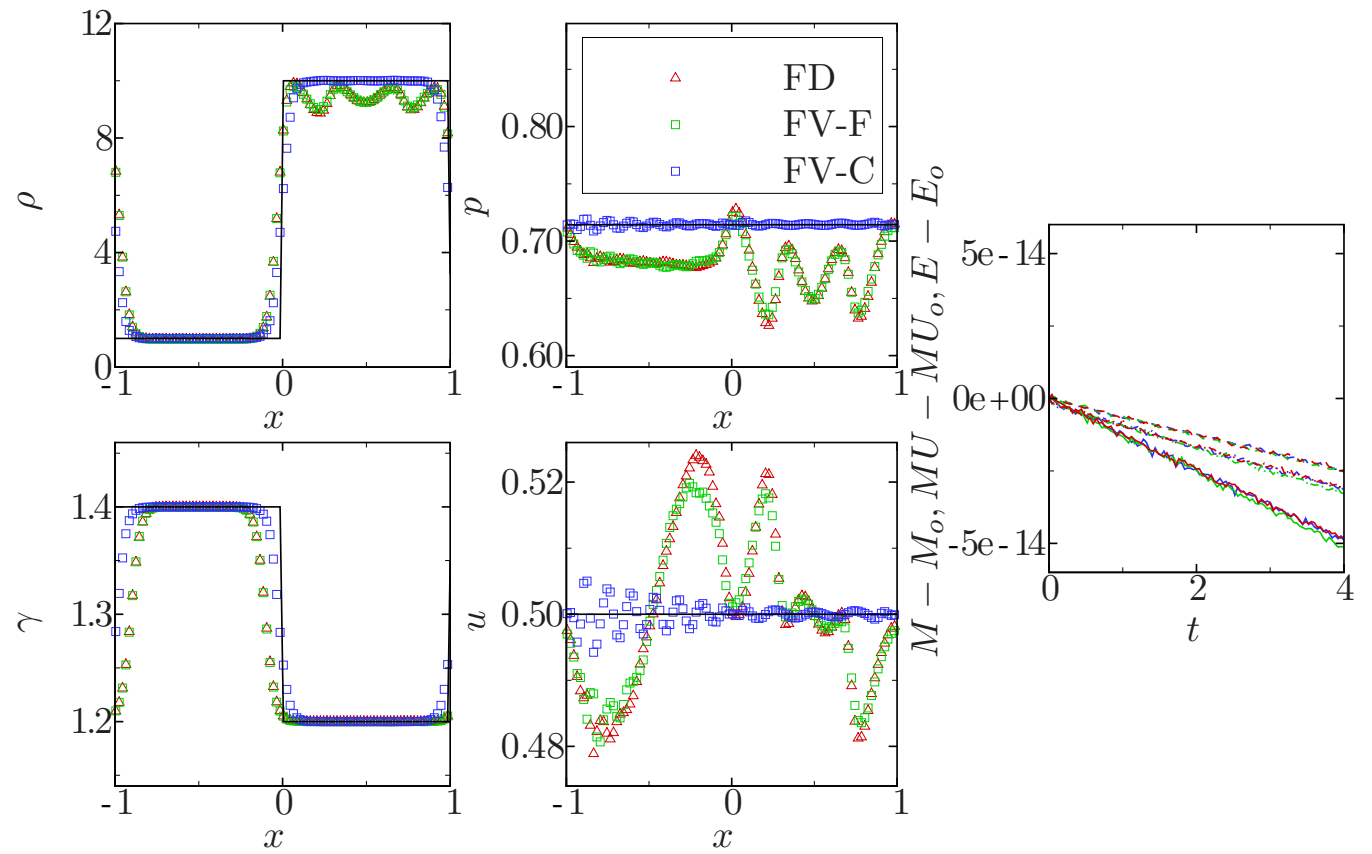

Figure 3.4: Density, pressure, $\gamma$, and velocity, along with the residual mass (dashed), momentum (dash-dot), and energy (solid), for the advection of a material interface with the FD, fully conservative FV (FV-F), and quasi-conservative FV (FV-C) schemes. The solid line denotes the exact solution.

are observed only at the round-off level when using the present scheme, as seen in Figure 3.5, where the base velocity and pressure have been subtracted. All schemes show discrete conservation to round-off error.

\subsubsection{Shock-tube problems}

Standard single-component shock-tube problems (Sod, 1978; Lax, 1954) were computed and produced good agreement amongst the schemes in Table 3.3. However, they are not shown here for conciseness. The present scheme with WENO5 and the HLLC solver is used, and $N=100$. 

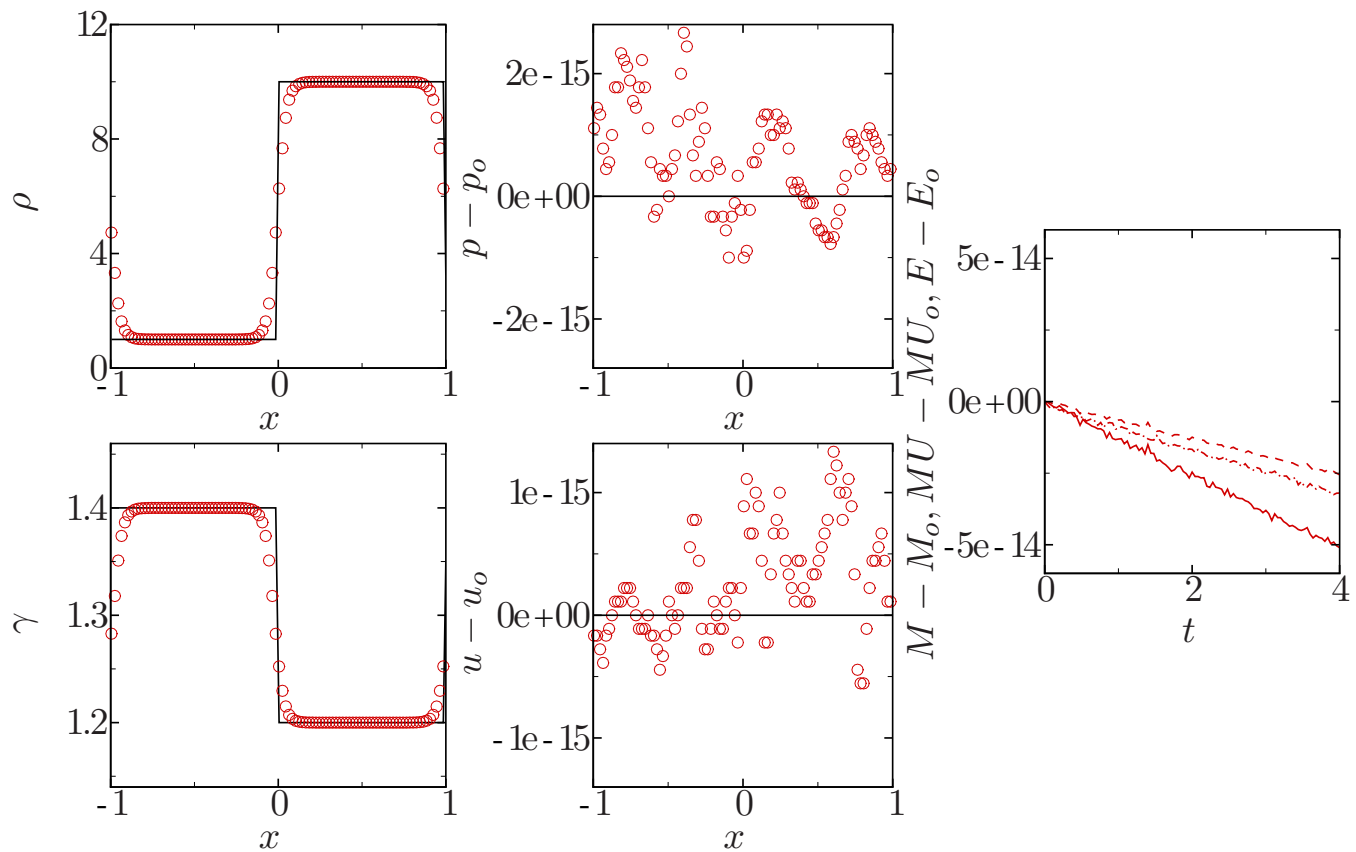

Figure 3.5: Density, pressure, $\gamma$, and velocity, along with the residual mass (dashed), momentum (dash-dot), and energy (solid), for the advection of a material interface with the present scheme (the base $u$ and $p$ have been subtracted). The solid line denotes the exact solution.

\section{Strong shock in gases}

The following multicomponent Sod problem with high pressure and density ratios (Wackers \& Koren, 2005) is considered:

$$
\left(\rho, u, p, \gamma, P_{\infty}\right)_{L}^{T}=(10,0,10,1.4)^{T}, \quad\left(\rho, u, p, \gamma, P_{\infty}\right)_{R}^{T}=(0.125,0,0.1,1.2)^{T}
$$

Figure 3.6 shows density, pressure, $\gamma$, and velocity profiles at time 1.6, as well as the residual mass, momentum, and energy, after subtraction of the total values.

Again, the computed solution agrees very well with the exact solution. Mass, momentum and energy are conserved to round-off error; the divergence of these values at large times illustrates that the shockwave is close to the boundary. In the $\gamma$ plot, there is more dissipation towards the left side of the interface, because of the 

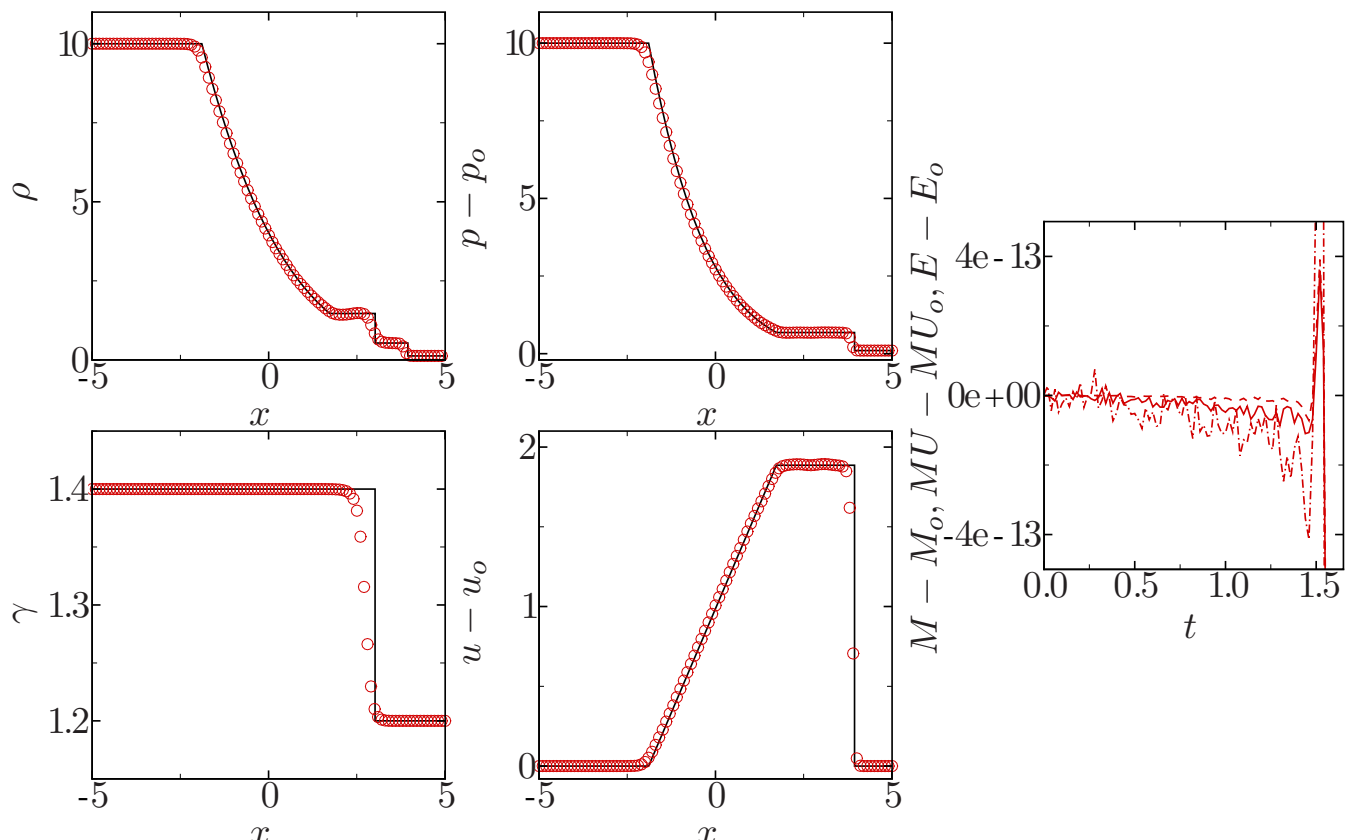

Figure 3.6: Density, pressure, $\gamma$, and velocity along with the residual mass (solid), momentum (dashed), and energy (dotted), for the modified Sod problem using the present scheme at time 1.6. The solid line denotes the exact solution.

original density ratio; although not shown here, as $N$ is increased, the computed solution converges to the exact solution.

\section{Gas-liquid Riemann problem}

A more difficult shock-tube problem is the following gas-liquid Riemann problem used to model underwater explosions (Cocchi et al., 1996; Shyue, 1998):

$$
\begin{aligned}
\left(\rho, u, p, \gamma, P_{\infty}\right)_{L}^{T} & =(1.241,0,2.753,1.4,0)^{T} \\
\left(\rho, u, p, \gamma, P_{\infty}\right)_{R}^{T} & =\left(0.991,0,3.059 \times 10^{-4}, 5.5,1.505\right)^{T} .
\end{aligned}
$$

The gas is highly compressed, as illustrated by the large pressure and small density ratios. Figure 3.7 shows density, pressure, $\gamma$, and velocity profiles at time 0.1 , as well as the residual mass, momentum, and energy after subtraction of the total 
values. The plot of $P_{\infty}$ is not included because it is similar to that of $\gamma$.
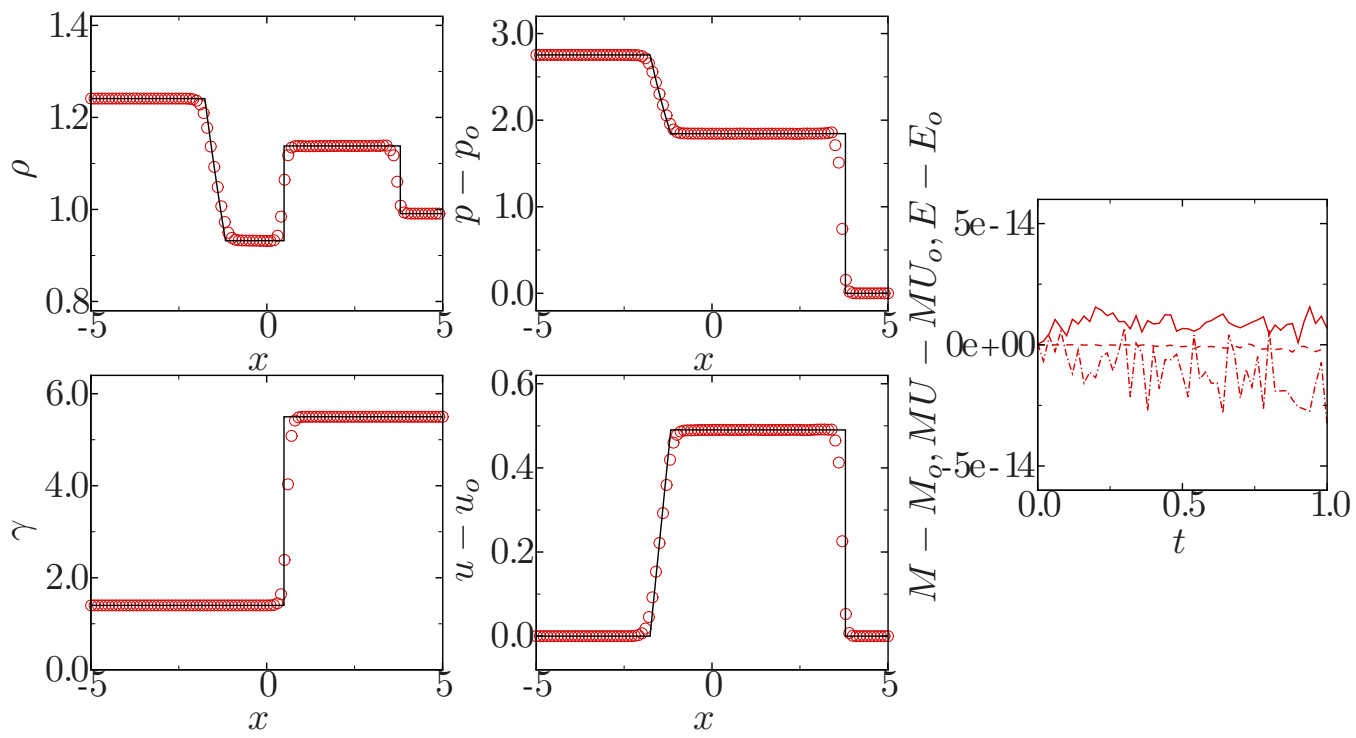

Figure 3.7: Density, pressure, $\gamma$, and velocity, along with the residual mass (solid), momentum (dashed), and energy (dotted), for the gas-liquid Riemann problem at time 0.1 . The solid line denotes the exact solution.

The computed solution agrees well with the exact solution and compares favorably to previous findings (Cocchi et al., 1996; Shyue, 1998). The wave speeds are correct and there are no oscillations at the interface. Mass, momentum, and energy are conserved up to round-off error.

\section{Shock-interface interaction}

The final one-dimensional test for oscillations and conservation is the interaction of a strong shock with an interface. A Mach 8.96 shockwave travels in helium and hits an air interface (Liu et al., 2003), with states:

$$
\begin{aligned}
(\rho, u, p, \gamma)_{L}^{T} & =(0.386,26.59,100,1.67)^{T} \\
(\rho, u, p, \gamma)_{M}^{T} & =(0.1,-0.5,1,1.67)^{T} \\
(\rho, u, p, \gamma)_{R}^{T} & =(1,-0.5,1,1.4)^{T}
\end{aligned}
$$


This shock is very strong, as illustrated by the pressure ratio of $100: 1$. Initially, the shock is located at $x=-0.8$, and the interface at $x=-0.2$. Figure 3.8 shows density, pressure, $\gamma$, and velocity profiles at time 0.07 , as well as the residual total mass, momentum, and energy after subtraction of the base values. The problem is solved using the present scheme with the Lax-Friedrichs solver.
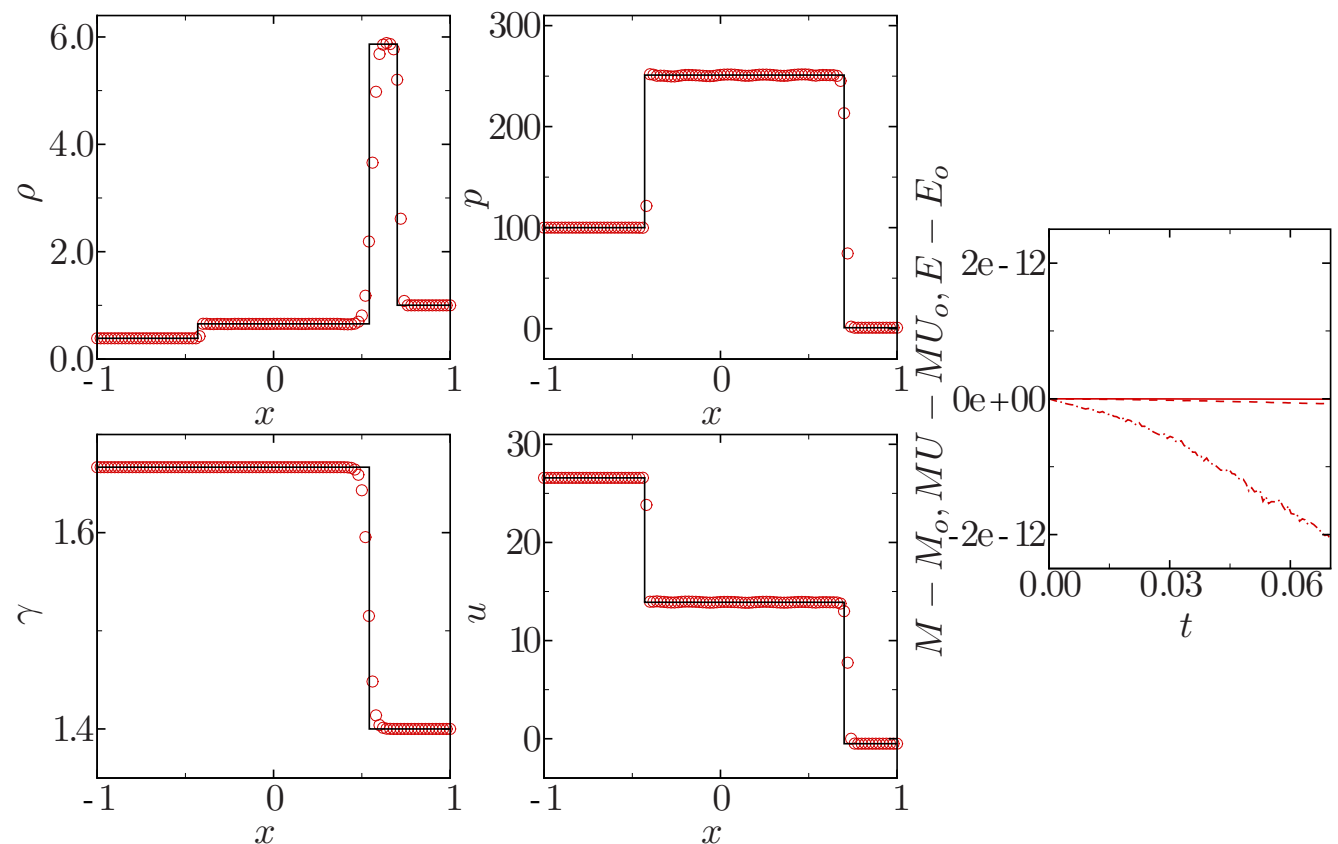

Figure 3.8: Density, pressure, $\gamma$, and velocity, along with the residual total mass (dashed), momentum (dash-dot), and energy (solid), for the shock-interface interaction at time 0.07 . The solid line denotes the exact solution.

Due to the respective impedances of the fluid components, one shock is reflected backwards, while another shock is transmitted into the air. The computed solution agrees well with the exact solution. The wave speeds are correct and there are no oscillations at the interface. Mass, momentum, and energy are conserved up to round-off error. 


\subsubsection{Shock-bubble interactions}

The previous examples show that the present method converges at the proper rate, and that the correct waves speeds are obtained in one-dimensional problems. The extension to two dimensions does not change this behavior. It is verified in Titarev \& Toro (2004) that the two-dimensional FV WENO schemes converge at the proper rate. The extension of Section 3.3.2 to two-dimensions is trivial, as the Euler equations are in flux-difference form and the reconstruction is conservative; therefore, for two-dimensional problems similar to the previous one-dimensional problems, conservation is satisfied.

An example of two-dimensional compressible multicomponent flow is the interaction between a shockwave and a cylindrical bubble studied experimentally in Haas \& Sturtevant (1987), where a Mach 1.22 shockwave in air impacts upon a Helium cylinder $\left(\rho_{H e}=0.138, \gamma_{H e}=1.67\right)$. The bubble has a $50 \mathrm{~mm}$ diameter, and the width of the shock-tube is $89 \mathrm{~mm}$. Several numerical studies have been undertaken, in which a non-conservative scheme with adaptive mesh refinement (Quirk \& Karni, 1996) and a FD-WENO5 scheme (Marquina \& Mulet, 2003) were used.

\section{One-dimensional problem}

The corresponding one-dimensional problem is first considered, to show convergence and to understand the early wave interactions. WENO5 with characteristic reconstruction is used, along with the HLLC solver; non-reflecting boundary conditions (Thompson, 1987) are implemented. Figures 3.9 and 3.10 show density, pres-

sure, $\gamma$, and velocity profiles shortly before the left-moving transmitted shockwave leaves the domain for the present and quasi-conservative FV schemes, respectively. Results with 100 and 400 points are presented in order to show convergence.

The density plots depict the interface and certain waves quite well. However, a better understanding of the wave interactions is achieved by considering the pressure and velocity plots. Initially, the incident shockwave hits the bubble from the left. Upon impact, a shockwave is transmitted into the bubble, whereas an expan- 

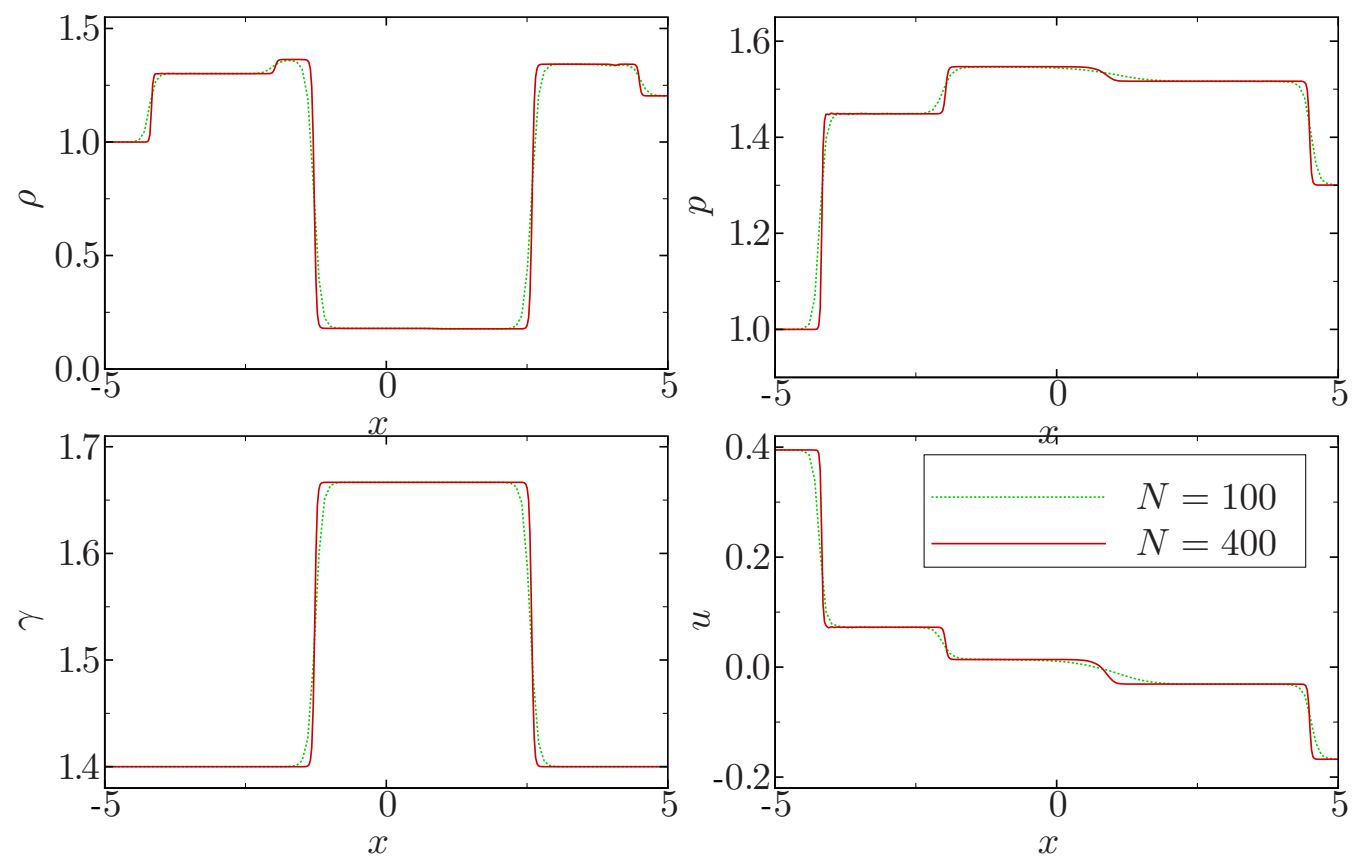

Figure 3.9: Density, pressure, $\gamma$, and velocity for the one-dimensional shock-bubble interaction using the present scheme (dotted: $N=100$; solid: $N=400$ ).

sion wave is reflected. The shockwave inside the bubble propagates to the other interface and, upon impact, generates a transmitted shockwave and a reflected shockwave. This phenomenon continues as the reflected shockwave trapped in the bubble impacts either interface. In Figures 3.9 and 3.10, the left-most wave is the first transmitted shockwave; the next wave is the second transmitted shockwave after two reflections off the interface; the next wave is the internally reflected shockwave after three reflections; and the right-most wave is the first transmitted shockwave after one reflection upon the far interface. The two left-most waves are propagating to the left and the two right-most to the right.

The quasi-conservative FV scheme clearly exhibits unacceptable oscillations, especially in the pressure and velocity. On the other hand, such oscillations do not occur when using the present scheme. Both schemes converge; the amplitude of the oscillations present in the former scheme decreases as the mesh is refined, but their frequency increases. 

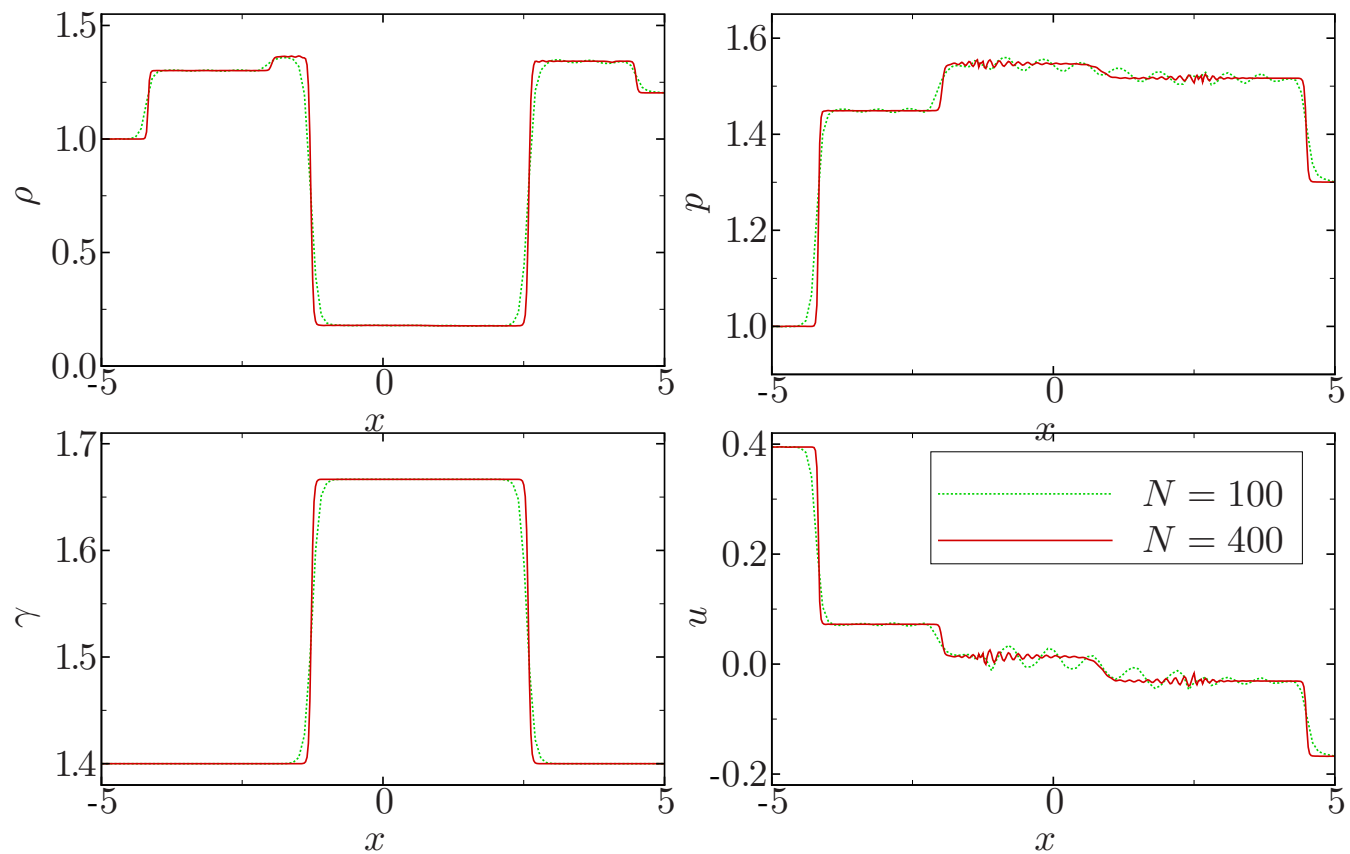

Figure 3.10: Density, pressure, $\gamma$, and velocity for the one-dimensional shockbubble interaction, using the quasi-conservative FV scheme (dotted: $N=100$; solid: $N=400)$.

\section{Two-dimensional problem}

For the two-dimensional problem, a $800 \times 400$ grid is used with reflecting boundary conditions along the top boundary and the centerline. The initial conditions are specified from a FV discretization. For a given radius, the appropriate properties are assigned inside and outside the bubble. Any cell that is crossed by the interface is given properties weighted by the fraction of both fluids. The initial shockwave is a straight discontinuity, with no smoothing.

Idealized Schlieren contours (Quirk \& Karni, 1996; Marquina \& Mulet, 2003), which allow the visualization of the general wave structure, and density lines, which show the details of the flow, are shown in Figure 3.11 for the present scheme. Frame A is taken shortly after the shockwave has impacted the bubble, frame B shortly before the first transmitted shockwave has left the domain, frame $\mathrm{C}$ shortly before 
the first reflection from the top wall leaves the domain, and frame $\mathrm{D}$ at a later time. The pressure along the horizontal centerline and the vertical line at the middle of the domain are plotted in Figure 3.12 at times corresponding to frames $\mathrm{A}$ and $\mathrm{B}$, respectively.

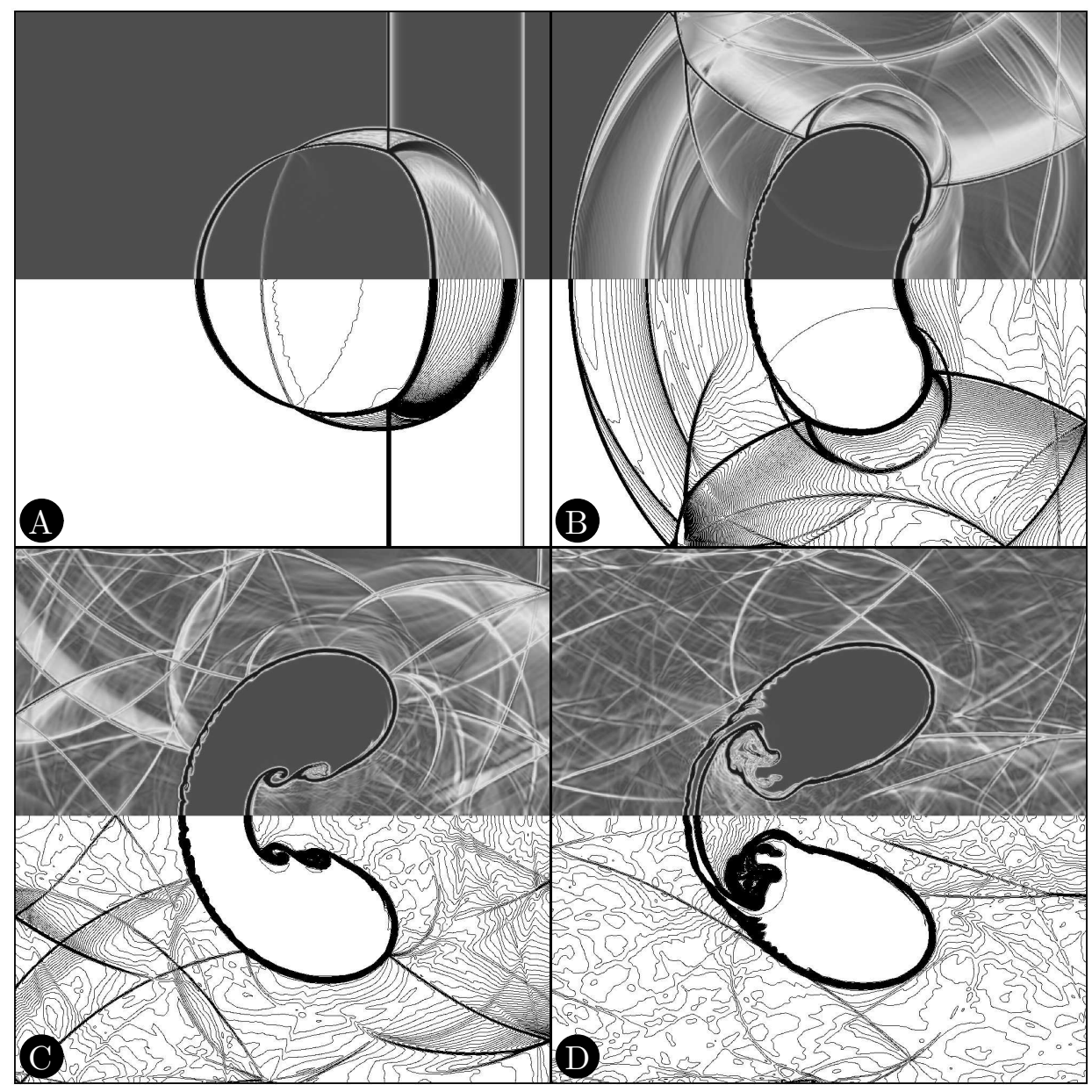

Figure 3.11: Idealized Schlieren images (top) and density lines (bottom) of an interaction between a Helium bubble and a Mach 1.22 shockwave in air using the present scheme.

The results are in good qualitative agreement with Haas \& Sturtevant (1987), Quirk \& Karni (1996) and Marquina \& Mulet (2003). The first wave interactions (frame A) and the subsequent reflections off the wall and interface (frame B) are 

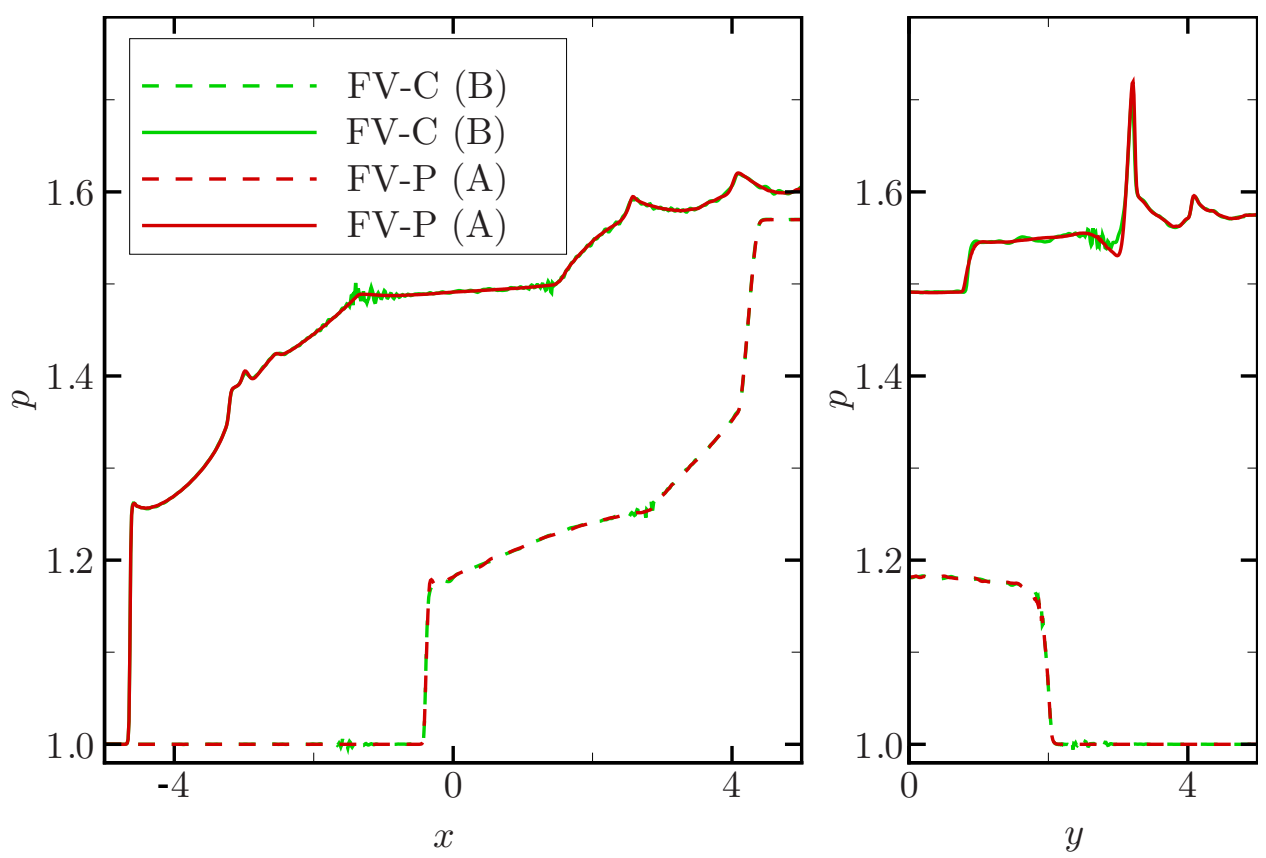

Figure 3.12: Pressure along the horizontal symmetry centerline $(y=0)$ on the left and the vertical centerline $(x=0)$ on the right corresponding to frames A (dotted) and B (solid) of Figure 3.11, using the present scheme and the quasi-conservative FV scheme.

well captured. The Kelvin-Helmholtz instability that develops along the interface and the jet formation (frames $\mathrm{C}$ and $\mathrm{D}$ ) are consistent with prior findings. The high-order accurate scheme allows the complex patterns due to the wave interactions to be well resolved; no spurious oscillations are observed at interfaces or shockwaves. The start-up error generated by the initial discontinuity is advected by the flow, but has no dynamical effect. This could have been avoided by starting the shockwave farther from the bubble (Quirk \& Karni, 1996) or by breaking a wave to form a shockwave.

On the other hand, Figure 3.13 shows that, when the quasi-conservative FV scheme is used, oscillations are generated at the interface in the form of spurious waves (frame A), as in the one-dimensional problem. Their main dynamical effect is to perturb the interface; this triggers the Kelvin-Helmholtz instability earlier than 


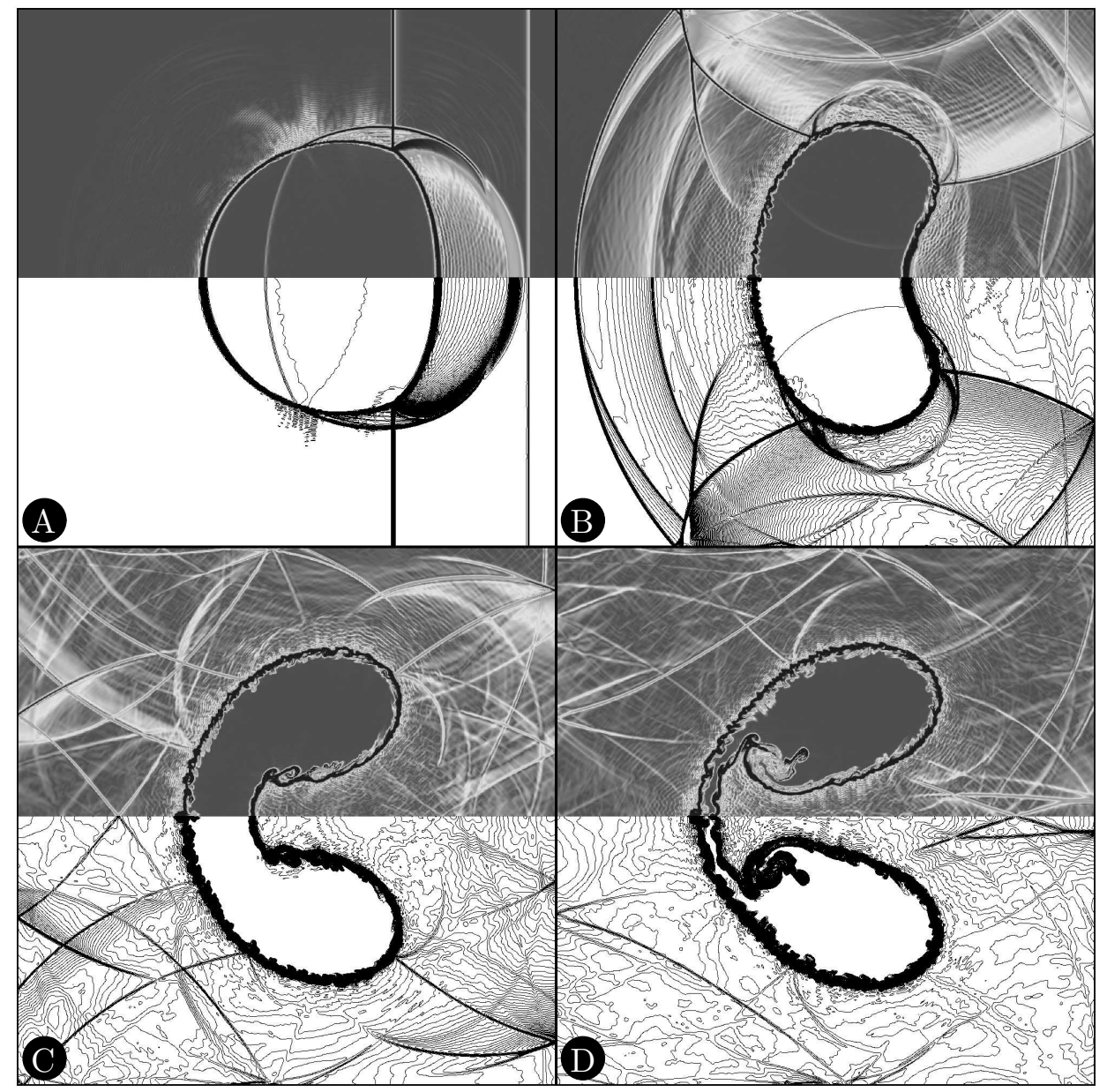

Figure 3.13: Idealized Schlieren images (top) and density lines (bottom) of an interaction between a Helium bubble and a Mach 1.22 shockwave in air, using the quasi-conservative FV scheme.

expected (frames B and C), as might be the case with the scheme of Marquina \& Mulet (2003), which does not strictly suppress oscillations. The interface becomes more smeared, which causes a decrease in the strength of the wave fronts that pass through it. These undesirable features do not occur when using the present method. The slight jaggedness of the two thin density lines inside the bubble in frame A for both cases is attributed to the initial irregular contour of the bubble on a Cartesian grid. 


\subsection{Extension of the numerical methodology}

\subsubsection{Finite volume equations in cylindrical coordinates}

Although a three-dimensional extension of the present numerical method is trivial, its implementation is computationally expensive. Instead, an axisymmetric extension is considered, since the bubble collapse problems considered here have azimuthal symmetry. For simplicity, only the radial component is considered; the axial component is treated as described in the previous sections.

In polar coordinates with azimuthal symmetry, the cell-average value of a function can be related to the cell-centered value using Taylor series:

$$
\frac{2 \pi}{\Delta V_{j}} \int_{r_{j-1 / 2}}^{r_{j+1 / 2}} f(r) r d r=\frac{F_{j+1 / 2}-F_{j-1 / 2}}{r_{j} \Delta r}=f_{j}+O\left(\Delta r^{2}\right),
$$

where $F^{\prime}=f r$. However, the following expression also approximates the cellcentered value of $f$ to the same order of accuracy:

$$
\frac{1}{\Delta r} \int_{r_{j-1 / 2}}^{r_{j+1 / 2}} f(r) d r \frac{1}{r_{j} \Delta r} \int_{r_{j-1 / 2}}^{r_{j+1 / 2}} r d r=f_{j}+O\left(\Delta r^{2}\right) .
$$

This naturally leads to the definition of a linear cell-average value, as in Equation 3.37 ,

$$
\bar{f}_{j}=\frac{1}{\Delta r} \int_{r_{j-1 / 2}}^{r_{j+1 / 2}} f(r) d r
$$

such that $f_{j} r_{j}=\overline{f r}_{j}+O\left(\Delta r^{2}\right)=\bar{f}_{j} \bar{r}_{j}+O\left(\Delta r^{2}\right)$. This follows the same idea as the appropriately averaged velocity and pressure described in Section 3.4.2. Then, the integral form of the radial component of Equations 2.2 can be re-arranged in 
the following semi-discrete fashion:

$$
\begin{aligned}
\frac{d}{d t} \bar{\rho}_{j} & =-\frac{(\rho v)_{j+1 / 2}-(\rho v)_{j-1 / 2}}{\Delta r}-\frac{(\rho v)_{j+1 / 2}+(\rho v)_{j-1 / 2}}{2 r_{j}}, \\
\frac{d}{d t} \overline{\rho v}_{j} & =-\frac{\left(\rho v^{2}+p\right)_{j+1 / 2}-\left(\rho v^{2}+p\right)_{j-1 / 2}}{\Delta r}-\frac{\left(\rho v^{2}\right)_{j+1 / 2}+\left(\rho v^{2}\right)_{j-1 / 2}}{2 r_{j}}, \\
\frac{d}{d t} \bar{E}_{j} & =-\frac{[v(E+p)]_{j+1 / 2}-[v(E+p)]_{j-1 / 2}}{\Delta r}-\frac{[v(E+p)]_{j+1 / 2}+[v(E+p)]_{j-1 / 2}}{2 r_{j}} .
\end{aligned}
$$

Thus, the right-hand side consists of a convective flux and a geometrical source term. The numerical fluxes obtained using the approximate Riemann solver can be used to compute the source terms in Equations 3.57a and 3.57c. For the momentum equation, the HLLC solver is applied to the variable, $\rho v^{2}$, in addition to the usual

flux, $\rho v^{2}+p$, so that appropriate upwinding is achieved for the source term. The form of Equations in 3.57 is similar to a finite difference discretization of the radial component of Equations 2.2.

\subsubsection{Boundary conditions at $r=0$}

In radial coordinates, the boundary conditions at the origin are similar to reflecting boundary conditions. The main difference is that the cell width is half that of a normal cell, as shown in Figure 3.14 below.

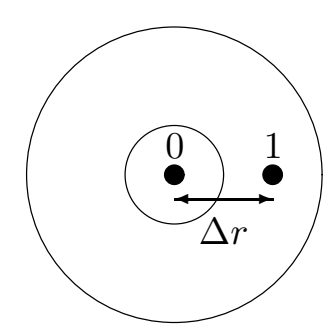

Figure 3.14: Schematic of the grid points near the centerline

The radial component of Equations 2.2 are integrated over the cell, so that, using the linear cell average from Equation 3.56, the following conditions are ob- 
tained at the centerline:

$$
\begin{gathered}
\frac{d}{d t} \bar{\rho}_{0}=-2 \frac{(\rho v)_{\Delta r / 2}}{\Delta r / 2}, \quad \frac{d}{d t} \overline{\rho u}_{0}=-2 \frac{(\rho u v)_{\Delta r / 2}}{\Delta r / 2}, \\
\frac{d}{d t} \overline{\rho v}_{0}=-2 \frac{\left(\rho v^{2}+p\right)_{\Delta r / 2}-\bar{p}_{0}}{\Delta r / 2}, \quad \frac{d}{d t} \bar{E}_{0}=-2 \frac{(v(E+p))_{\Delta r / 2}}{\Delta r / 2}, \\
\frac{d}{d t} \bar{\Gamma}_{0}=0,
\end{gathered}
$$

where $\bar{p}_{0}$ is computed from the cell-average values of the conservative variables.

\subsubsection{Computational domain}

\section{Domain size}

Non-reflecting boundary conditions are employed to truncate the computational domain and extend it to infinity (see Section 3.5). However, the scheme used in the present work (Thompson, 1987, 1990) is not exact in multidimensions, so that spurious waves are reflected back into the domain (Colonius, 2004). Although these reflections are weak at the boundaries, they focus in the cylindrical geometry. Thus, the domain is extended to a size large enough that the waves reflected at the boundaries do not have time to contaminate the solution. Since these waves travel at sonic speeds, the length of the domain is set by computing the distance traveled by waves emitted during bubble collapse over the time of the collapse (computed from the Rayleigh-Plesset equation 4.4). Furthermore, the stretching described in the next section allows the outgoing waves to dissipate significantly on the coarse grid far away from the bubble. Another way to prevent reflections is to implement a buffer region (Colonius \& Ran, 2002).

\section{Mesh stretching}

As remarked in the previous section, the domain must be large enough to prevent reflections off the boundaries from contaminating the solution. Since the area of interest is small, a static stretched grid is used to minimize the computational effort. 
In order to prevent reflections due to an abrupt change in grid spacing, the following infinitely smooth function is used:

$$
h(x)=2 x_{0}-\left(x_{1}+x_{2}\right)+\frac{\ln \left[\cosh \left(a\left(x-x_{1}\right)\right)\right]+\ln \left[\cosh \left(-a\left(x+x_{2}\right)\right)\right]}{a},
$$

where $x_{0}$ is determined by setting $h(0)=1$. In the case of free-field Rayleigh collapse, $a=4$ and $x_{1}=x_{2}=1.83$. Figure 3.15 shows the grid-stretching function.

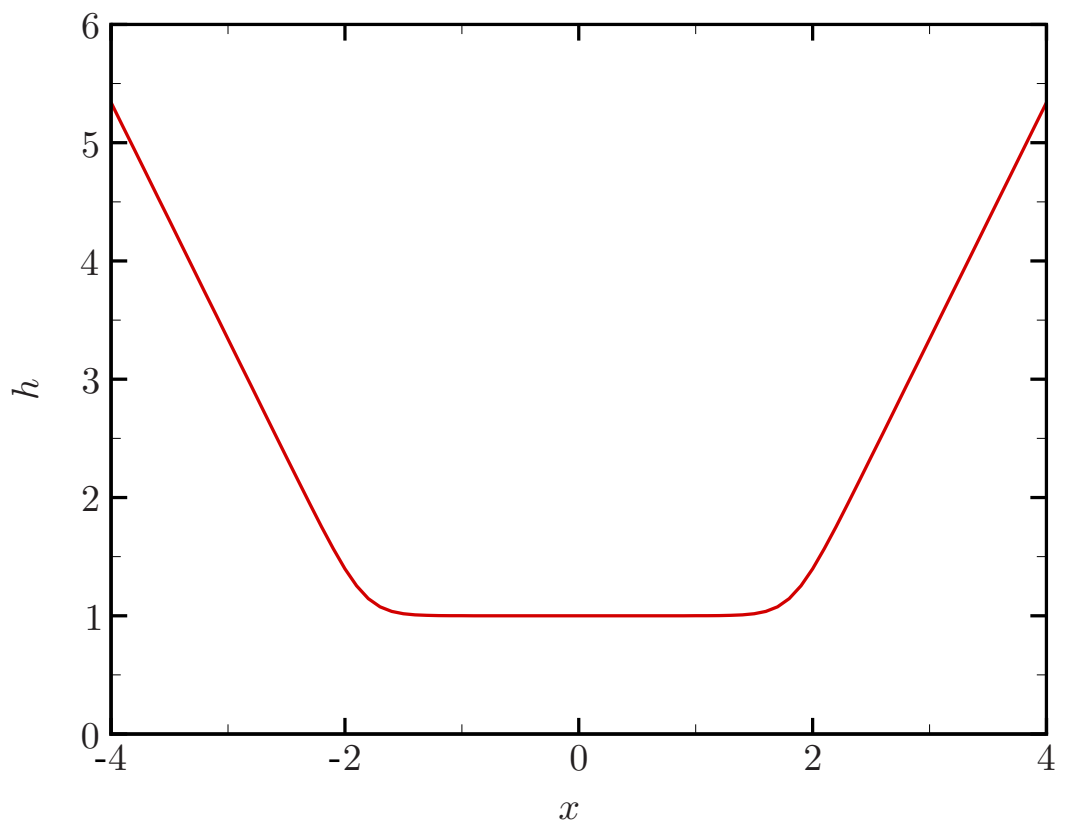

Figure 3.15: Grid-stretching function.

The resulting grid allows for high resolution near the bubble and a coarse mesh far away. Because of the stretching, the grid is no longer uniform, as illustrated in Figure 3.16, which shows the computational domain for the free-field Rayleigh collapse (only one in ten points is included). Thus, the WENO scheme is extended to account for the non-uniformity. In particular, the modified form of the polynomials, the ideal weights, and the smoothness indicators were calculated based on the grid, according to the equations listed in Appendix B. On the other hand, the multidimensional reconstruction (Titarev \& Toro, 2004), artificial compression (Yang, 1990), and mapped-WENO scheme (Henrick et al., 2005) were not extended 
to non-uniform grid.

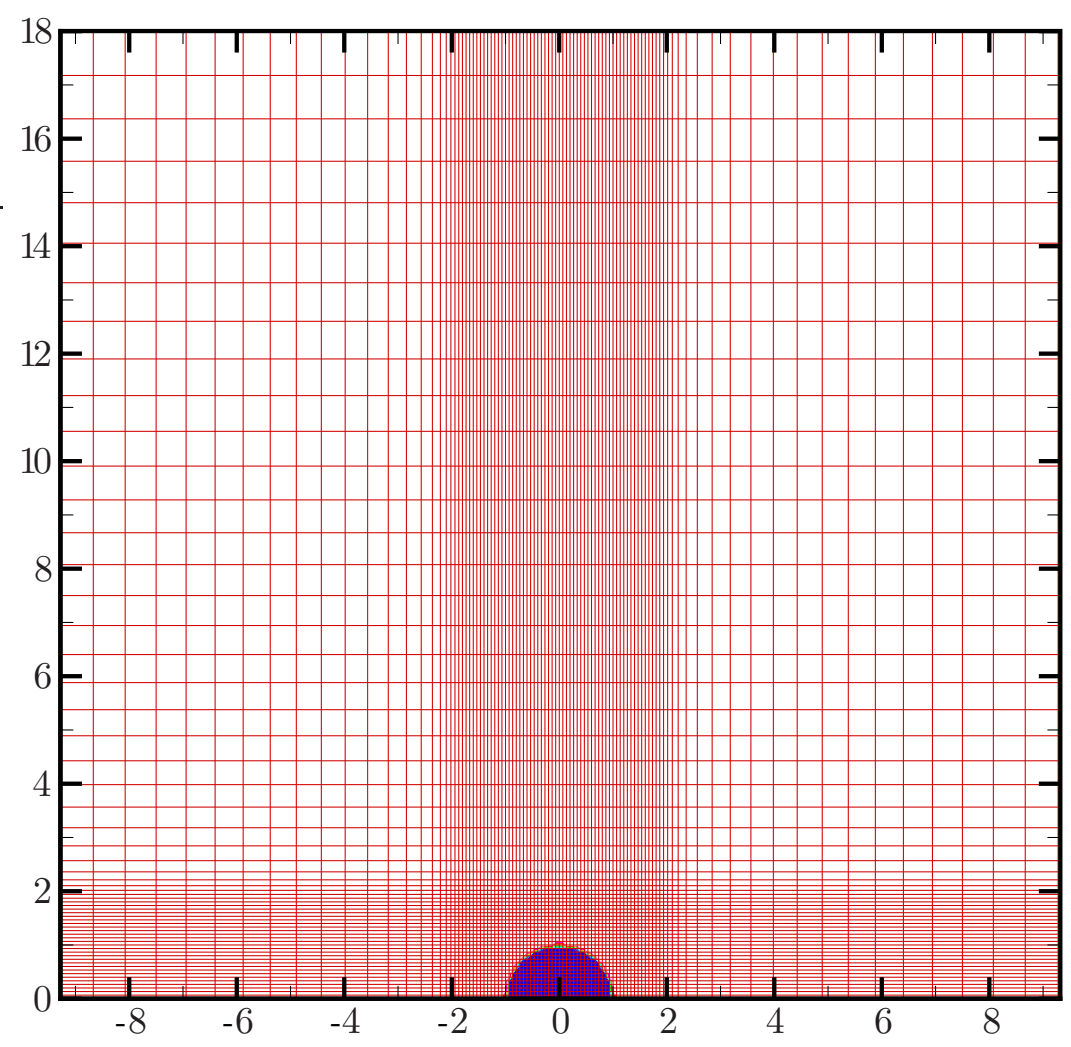

Figure 3.16: Computational grid used for Rayleigh collapse (only one in ten cells is shown.

\subsubsection{Initial conditions}

\section{Volume fraction}

The initial conditions for computational cells containing only gas or liquid are easily specified. However, for cells that are intersected by the interface, the variables must be assigned based on the fraction of gas and of liquid contained in the cell. By definition, the volume fraction of gas is defined as $\xi_{i j}=\frac{V_{G}}{V_{i j}}$. Since the density in a cell is given by the total mass of fluid divided by the cell volume, it follows that 
the density of a cell is given by

$$
\rho_{i j}=\xi_{i j} \rho_{G}+\left(1-\xi_{i j}\right) \rho_{L}
$$

Similarly, the impedance in a mixture is given by (Brennen, 1995):

$$
\frac{1}{\rho c^{2}}=\frac{x}{\rho_{G} c_{G}^{2}}+\frac{1-x}{\rho_{L} c_{L}^{2}}
$$

From Equations 3.60 and 3.61, the local mixture value of $\gamma$ and $P_{\infty}$ can be specified:

$$
\frac{1}{\gamma_{i j}}=\frac{\xi_{i j}}{\gamma_{G}}+\frac{1-\xi_{i j}}{\gamma_{L}}, \quad P_{\infty, i j}=P_{\infty, L}
$$

\section{Initial smoothing of the interface}

In the finite volume formulation, the initial conditions for the bubble can be specified based on the volume fraction of gas in each cell. However, this leads to corrugations of the bubble shape, due to the discretization of a circle onto a rect-

angular grid, as described in Section 3.8. Thus, the volume fraction function was smoothed over a few grid points using the following function:

$$
\xi_{i j}=\frac{1-\tanh \left[\alpha\left(\sqrt{x_{i}^{2}+r_{j}^{2}}-R_{o}\right) / \Delta x\right]}{2},
$$

where the coefficient, $\alpha \approx 1-10$, such that the bubble volume and circulation do not change significantly.

\subsection{Limitations of the numerical scheme}

\subsubsection{Inviscid simulations}

The numerical solution to the multidimensional Euler equations in which shockwaves interact with interfaces may exhibit ill-posed features. In particular, Samtaney \& Pullin (1996) showed that the solution to the interaction between a shock and a vortex sheet does not necessarily converge in a pointwise or weak sense. 
As discussed in Niederhaus et al. (2007), irregularities due to the discretization of the initial conditions introduce a cut-off wavenumber for the Kelvin-Helmholtz instability, so that the solution does not continuously depend on the initial conditions. In the limit of infinite resolution, the wavenumber of these perturbations tends to infinity, leading to the exponential growth characteristic of the Hadamard instability (Joseph, 1989).

Such ill-posedness is not exhibited in nature, because of the regularizing effects of viscosity, surface tension, and other diffusive processes. From a numerical standpoint, this problem may be lessened by initially smoothing interfaces over a few grid points (Niederhaus et al., 2007), as described in Section 3.7.4. It should be noted that both the shock- and interface-capturing capability of the present method introduce numerical dissipation at shockwaves and interfaces, thereby numerically smoothing gradients.

In order to illustrate such features, Figure 3.17 shows contours of vorticity and lines of density for shock-induced collapse with $p_{s} / p_{o}=353$. Different resolutions are included $\left(\Delta x_{\min }=2.67 \times 10^{-2}, 1.33 \times 10^{-2}, 6.67 \times 10^{-3}\right)$. Only the half part of the bubble is shown, 5.49 time units after the shock hit the bubble; evidence of the transmitted shock can be seen in the vorticity plots.

As explained in Section 4.3.9, baroclinic vorticity is generated as the shock intersects the interface, due to the misalignment of the density and pressure gradients. In theory, only negative (clockwise) vorticity should be generated during the process. However, positive vorticity is observed in Figure 3.17, especially near the axis, $r=0$. This can be attributed to the fact that grid-dependent perturbations affect the small baroclinic term in this region. The perturbations wavelength depends on the grid-spacing. As the grid is refined, the amplitude of the perturbations increases, but the area over which they grow decreases. As a result, the overall circulation is not affected by this, as shown in Figure 3.18. 

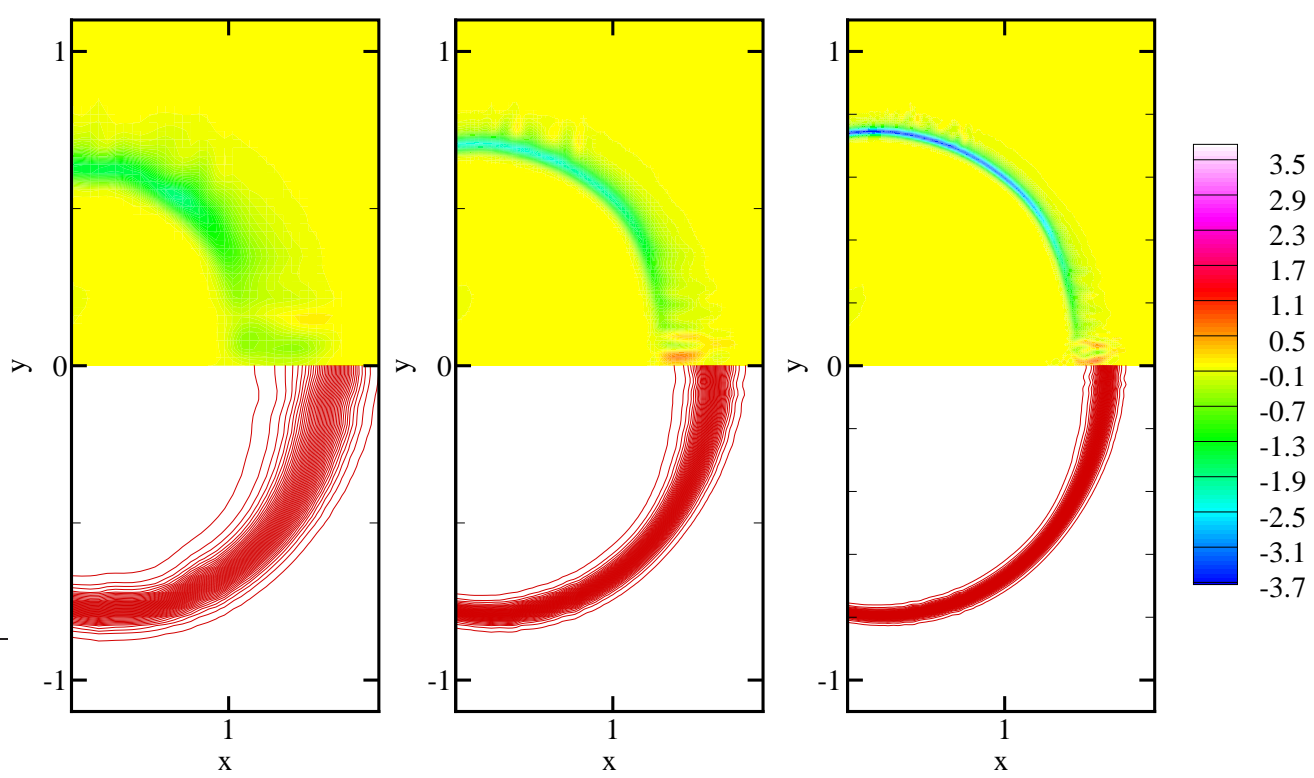

Figure 3.17: Vorticity contours (top) and density lines (bottom) for shock-induced collapse $\left(p_{s} / p_{o}=641\right)$ for different resolutions (left: $\Delta x_{\min }=2.67 \times 10^{-2}$; center: $\Delta x_{\min }=1.33 \times 10^{-2}$; right: $\left.\Delta x_{\min }=6.67 \times 10^{-3}\right)$.

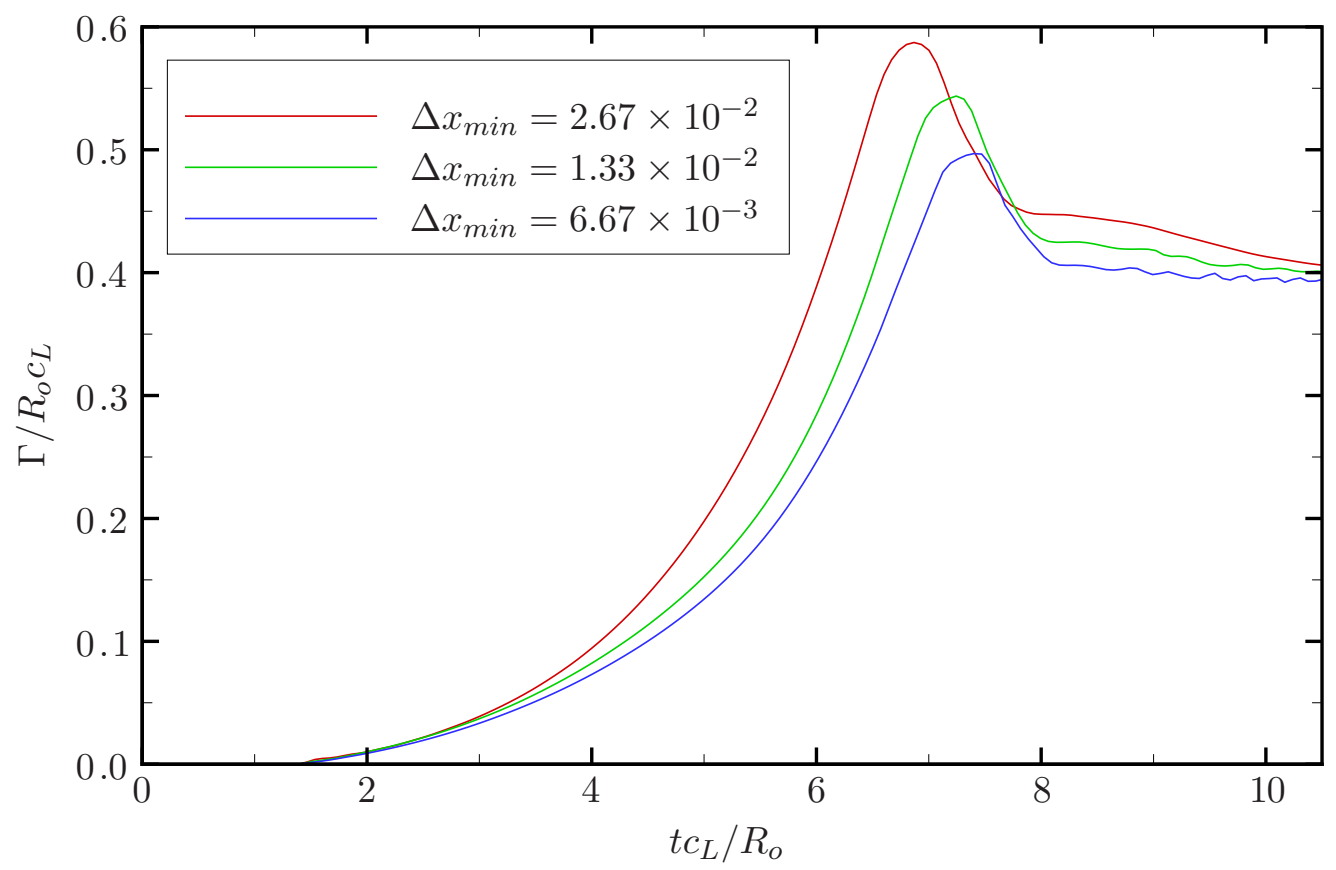

Figure 3.18: Circulation history for different resolutions. 


\subsubsection{Resolution}

For smooth problems, Section 3.6.1 showed good convergence properties. However, when discontinuities such as shockwaves and interfaces are present, the method reduces to first-order accuracy in those regions. As a result, a far greater number of grid points are required in order to make meaningful predictions.

In order to illustrate this issue, Figure 3.19 shows the history of the bubble volume for free-field Rayleigh collapse $\left(p_{s} / p_{o}=353\right)$ for different resolutions until the bubble reaches $V_{\min }$.

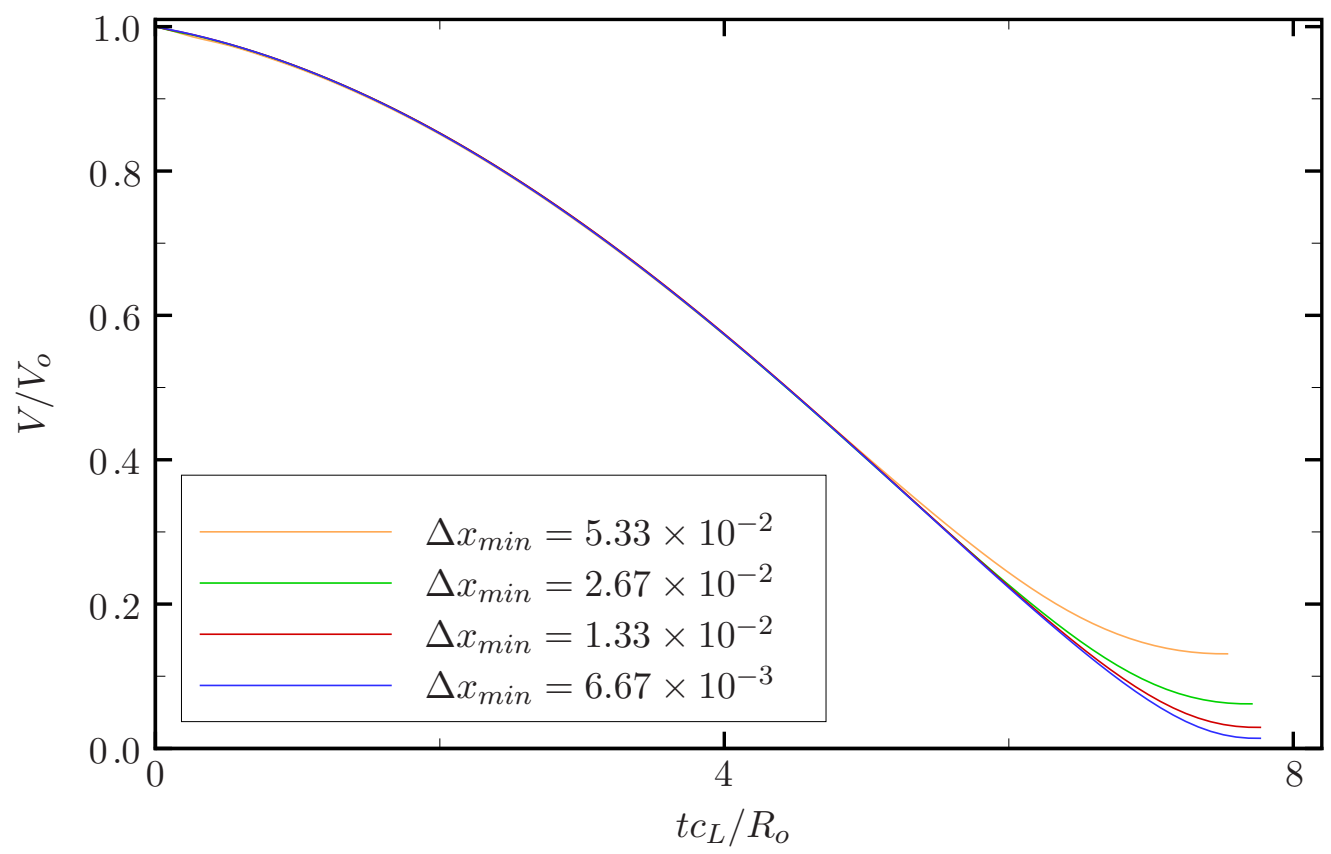

Figure 3.19: Bubble volume history for different resolutions $\left(p_{s} / p_{o}=353\right)$.

As the number of grid points is increased, the bubble volume appears to be converging. ${ }^{4}$ Even though convergence is not completely achieved, the present computations constitute the best possible effort based on the current computing power to obtain solutions that can be used to carry out a parameter study.

\footnotetext{
${ }^{4}$ Although it was noted in Section 3.8 that pointwise convergence may not hold, the smoothing introduced in Section 3.7.4 alleviates this ill-posedness, so that convergence does have a meaning in the present computations.
} 
Because the grid is not aligned with the bubble interface, a shape instability can be observed in idealized Schlieren contours; such grid-dependent effect may be relevant to the instabilities shown in Nagrath et al. (2006). This feature depends on the numerical dissipation of the scheme and therefore decreases as the resolution is increased; this matter is further discussed in Section 4.3.3. Figure 3.20 depicts density lines and Schlieren contours at different resolutions for free-field Rayleigh collapse $\left(p_{s} / p_{o}=353\right)$.
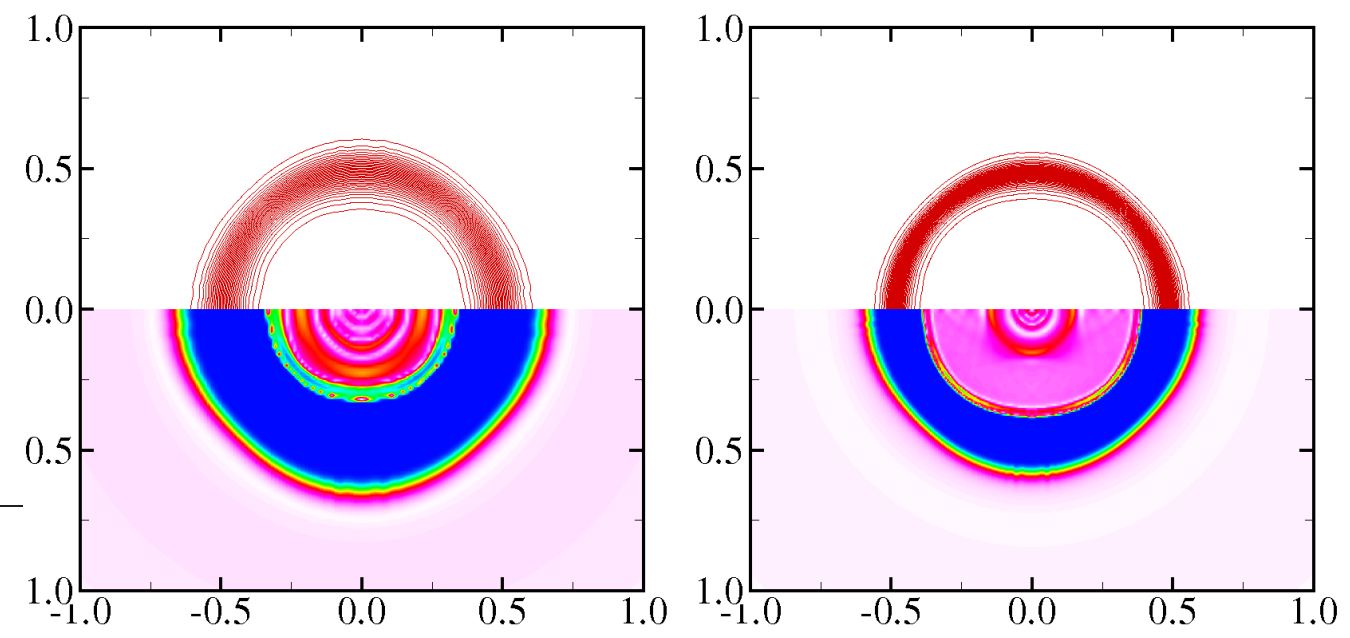

Figure 3.20: Density lines (top) and numerical Schlieren contours (bottom) for $\Delta x=1.33 \times 10^{-2}$ (left) and $\Delta x=6.67 \times 10^{-3}$ (right).

There appears to be a preferred direction of dissipation in the Schlieren contours (at $\sim 45^{\circ}$ ). This may be explained by the fact that the numerical fluxes are evaluated in the $x$ and $r$ directions separately, so that flow features at an angle of $45^{\circ}$ receive contributions from both directions. Yet, the density lines are less distorted. Schlieren plots are useful because they allow both interfaces and shockwaves to be visualized. This quantity is computed from the derivative of density (see Section C.5), so that such effects are emphasized, and the interface tends to look more diffuse than it really is.

The resolution also plays a role in the interfacial region. Because of the numerical diffusion in the interface-capturing scheme, the interface spreads over a 
few grid points and thus has a finite thickness. The diffuse interface consists of a mixture of gas and liquid with properties $\left(\gamma\right.$ and $\left.P_{\infty}\right)$ given based on the fraction of each phase. This effect may become problematic when a large negative pressure interacts with the interface, because the stiffness in this region can be small, thereby leading to negative values of $c^{2}$ and stopping the simulation. This phenomenon sometimes occurs in the present simulations, but usually long after collapse, so that the results are not affected.

\subsection{Summary of the numerical method}

A high-order accurate, quasi-conservative, shock- and interface-capturing scheme was developed to simulate compressible multicomponent flow problems. A TVD Runge-Kutta method is used for time-marching, while the spatial discretization consists of a finite volume WENO scheme, where the average primitive variables are reconstructed, and the HLLC solver, which is modified to solve the advection equations.

The following algorithm is proposed to compute compressible multicomponent flows based on the model equations 2.1 and 2.8:

Given the cell averages, $\overline{\mathbf{q}}_{i}$ and $\bar{\phi}_{i}$, at some time step, $n$,

1. Build the average primitive variables, $\overline{\mathbf{u}}_{i}$,

2. Using WENO, reconstruct the primitive variables $\mathbf{u}_{i+1 / 2}^{L}$ and $\mathbf{u}_{i+1 / 2}^{R}$,

3. Build the conservative variables $\mathbf{q}_{i+1 / 2}^{L}$ and $\mathbf{q}_{i+1 / 2}^{R}$ and the fluxes $\mathbf{f}_{i+1 / 2}^{L}$ and $\mathbf{f}_{i+1 / 2}^{R}$

4. Use the HLLC solver to compute the numerical flux, $\mathbf{f}_{i+1 / 2}$,

5. Use the adapted HLLC solver to compute the right-hand side of the advection equations, and

6. March $\overline{\mathbf{q}}_{i}$ and $\bar{\phi}_{i}$ forward in time.

The reconstruction is applied to the local characteristic fields (Shu, 1999), with the transformation matrices based on the primitive variables. This is done 
by multiplying $\overline{\mathbf{u}}_{i}$ by the left Roe matrix after step one, and $\mathbf{u}_{i+1 / 2}^{L}$ and $\mathbf{u}_{i+1 / 2}^{R}$ by the right Roe matrix after step two, where the matrices are given by Equation 2.5. This procedure requires approximately the same number of operations as a standard WENO code, and is no more difficult to implement.

Verification was carried out using one-dimensional Riemann problems and twodimensional shock-bubble interaction in gases. The appropriate convergence rate was achieved in smooth problems, no oscillations were generated at discontinuities, and mass, momentum and energy were conserved to round-off levels.

The method is extended to a non-uniform grid and cylindrical coordinates with azimuthal symmetry, in order to simulate bubble collapse problems. It is noted that certain grid-dependent features may occur, but do not affect the results significantly. 


\section{Chapter 4}

\section{Dynamics of non-spherical bubble collapse}

Results from the numerical simulations of Rayleigh collapse and shock-induced collapse in a free-field and near a wall are discussed in the present chapter. First, relevant analytical findings from spherical bubble dynamics are derived. Then, in order to provide a qualitative understanding of the important features, the flow field is visualized using contour plots. Next, the dynamics of bubble collapse are considered in detail by examining a wide range of properties. Based on these observations, the jet formation mechanism is discussed. Finally, the potential damage caused by the shockwaves emitted during bubble collapse is studied.

\subsection{Preamble: spherical bubble dynamics}

Although the collapse in most cases of interest is non-spherical, spherical bubble dynamics and the Rayleigh-Plesset equation are briefly discussed in order to understand certain fundamental aspects.

\subsubsection{Rayleigh-Plesset equation}

The derivation of the Rayleigh-Plesset equation for spherical bubble collapse follows that of Brennen (1995). The domain, at the origin of which the bubble is located, consists of an infinite sea of liquid, with boundary conditions at infinity,

$u(\infty, t)=0, p(\infty, t)=p_{\infty}(t)$. The flow is assumed incompressible in the liquid, so that, using the kinematic boundary condition at the interface, the velocity field 
can be written $u(r, t)=\frac{R^{2}}{r^{2}} \dot{R}$, where $R(t)$ is the bubble radius. Since this holds anywhere in the liquid, the momentum equation,

$$
\frac{\partial u}{\partial t}+u \frac{\partial u}{\partial r}=-\frac{1}{\rho_{L}} \frac{\partial p}{\partial r}
$$

can be integrated from infinity to the bubble interface to yield

$$
-\frac{p_{\infty}(t)-p(R)}{\rho_{L}}=\ddot{R} R+\frac{3}{2} \dot{R}^{2} .
$$

This equation is valid at the interface, where the pressure difference is balanced by viscous and surface tension stresses: $p_{B}=p(R, t)+4 \mu_{L} \dot{R} / R-2 S / R$, where $\mu_{L}$ is the dynamic viscosity and $S$ is the surface tension. If the bubble is assumed to contain vapor at ambient temperature and non-condensible gas obeying an adiabatic law, then the generalized Rayleigh-Plesset equation can be written

$$
\frac{p_{V}\left(T_{\infty}\right)-p_{\infty}(t)}{\rho_{L}}+\frac{p_{G o}}{\rho_{L}}\left(\frac{R_{o}}{R}\right)^{3 \gamma}=R \ddot{R}+\frac{3}{2} \dot{R}^{2}+\frac{4 \nu_{L} \dot{R}}{R}+\frac{2 S}{\rho_{L} R},
$$

where $p_{V}$ is the saturation pressure, $p_{G o}$ is the pressure of the non-condensible gas, and $\nu_{L}$ is the kinematic viscosity. It is noted that the presence of vapor simply provides a reduction in the pressure jump across the bubble interface. This equation was first derived by Rayleigh (1917), who assumed a constant pressure at infinity and neglected viscosity and surface tension, and later extended by Plesset (1949) to include these effects.

In the present work, viscous and surface tension effects are neglected, the bubble is assumed to contain only air, and the pressure is constant at infinity. ${ }^{1}$ Thus, the following equation constitutes the model for the spherical collapse studies in the present work:

$$
R \ddot{R}+\frac{3}{2} \dot{R}^{2}=\frac{p_{G o}}{\rho_{L}}\left[\left(\frac{R_{o}}{R}\right)^{3 \gamma}-\frac{p_{\infty}}{p_{G o}}\right] .
$$

\footnotetext{
${ }^{1}$ Based on these assumptions and on the problem description of Section 2.1, $p_{\text {Go }}=p_{a t m}$ in the present case, so that the relevant pressure ratio is $p_{\infty} / p_{a t m}$.
} 
Noting that

$$
\frac{d}{d t}\left(R^{3} \dot{R}^{2}\right)=2 R^{3} \dot{R} \ddot{R}+3 R^{2} \dot{R}^{3}=2 R^{2} \dot{R}\left(R \ddot{R}+\frac{3}{2} \dot{R}^{2}\right)
$$

Equation 4.4 can be written:

$$
\frac{1}{2 R^{2} \dot{R}} \frac{d}{d t}\left(R^{3} \dot{R}^{2}\right)+\frac{p_{G o}}{\rho_{L}}-\frac{p_{G o}}{\rho_{L}}\left(\frac{R_{o}}{R}\right)^{3 \gamma}=0
$$

Since $R=R(t)$ and $\dot{R}=\dot{R}(t)$, then $\dot{R}^{-1} d / d t=d / d R$. Thus, the Rayleigh-Plesset equation can be integrated from $R_{o}$ to $R$ to yield ${ }^{2}$

$$
\dot{R}^{2}=\frac{2}{3} \frac{p_{G o}}{\rho_{L}}\left[\frac{1}{\gamma-1}\left(\frac{R_{o}}{R}\right)^{3 \gamma}-\frac{p_{\infty}}{p_{G o}}+\left(\frac{p_{\infty}}{p_{G o}}-\frac{1}{\gamma-1}\right)\left(\frac{R_{o}}{R}\right)^{3}\right]
$$

Alternately, this expression can be derived by equating the kinetic energy of motion in the liquid to the work done on the liquid by the bubble. Neglecting gas contents, Rayleigh (1917) obtained an expression for the time it takes for the bubble to collapse to zero size:

$$
\tau_{c}=0.915 \sqrt{\frac{\rho_{L}}{p_{\infty}}} R_{o}
$$

More generally, the ordinary differential equation 4.4 can be integrated numerically to obtain $R(t)$. Figure 4.1 shows the history of the radius and of the interface velocity for the Rayleigh collapse of a gas bubble with $p_{s} / p_{o}=p_{\infty} / p_{G o}=34$. The acceleration at early times and at collapse is depicted in Figure 4.2.

The initial collapse is driven by the pressure difference between the bubble and infinity; $R(t)$ changes slowly, since both $\dot{R}$ and $\ddot{R}$ are small in Equation 4.4. After some time, the nonlinear velocity term becomes important, leading to a rapid increase in the bubble wall velocity. Soon after, the gas pressure increases substantially due to adiabatic compression and overwhelms the other terms, causing a large deceleration. As a result, the collapse is arrested and rebound ensues. Be-

\footnotetext{
${ }^{2}$ The initial condition, $\dot{R}(0)=0$, is used here. If the liquid is compressible, the initial interface velocity is non-zero and can be approximated by $\dot{R}(0) \approx-\left(p_{s}-p_{o}\right) / \rho_{L} c_{L}^{2}$ (Plesset \& Prosperetti, 1977) in the case of Rayleigh collapse. See Section 4.3.2 for more details.
} 

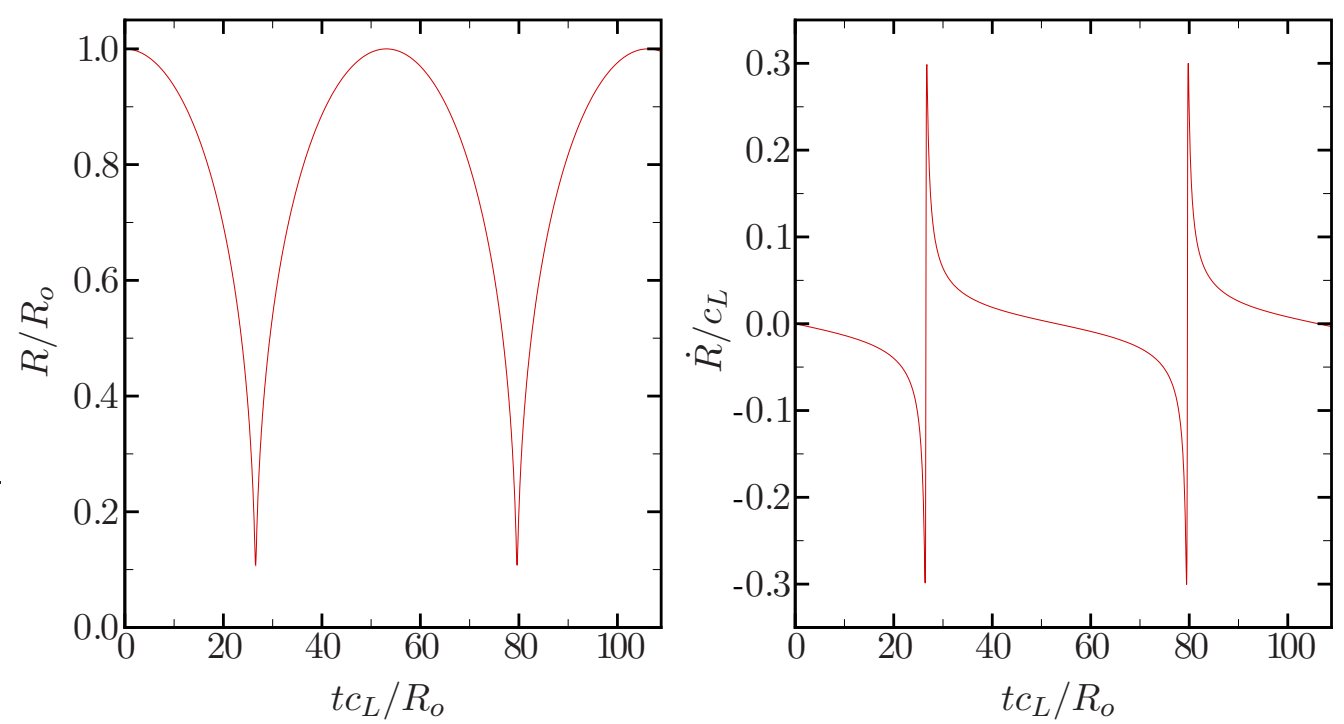

Figure 4.1: Solution to the Rayleigh-Plesset equation for Rayleigh collapse $\left(p_{s} / p_{o}=34\right)$. Left: bubble radius; right: interface velocity.

cause there are no mechanisms for energy loss in Equation 4.4, the bubble rebounds to its initial radius and the process is repeated.
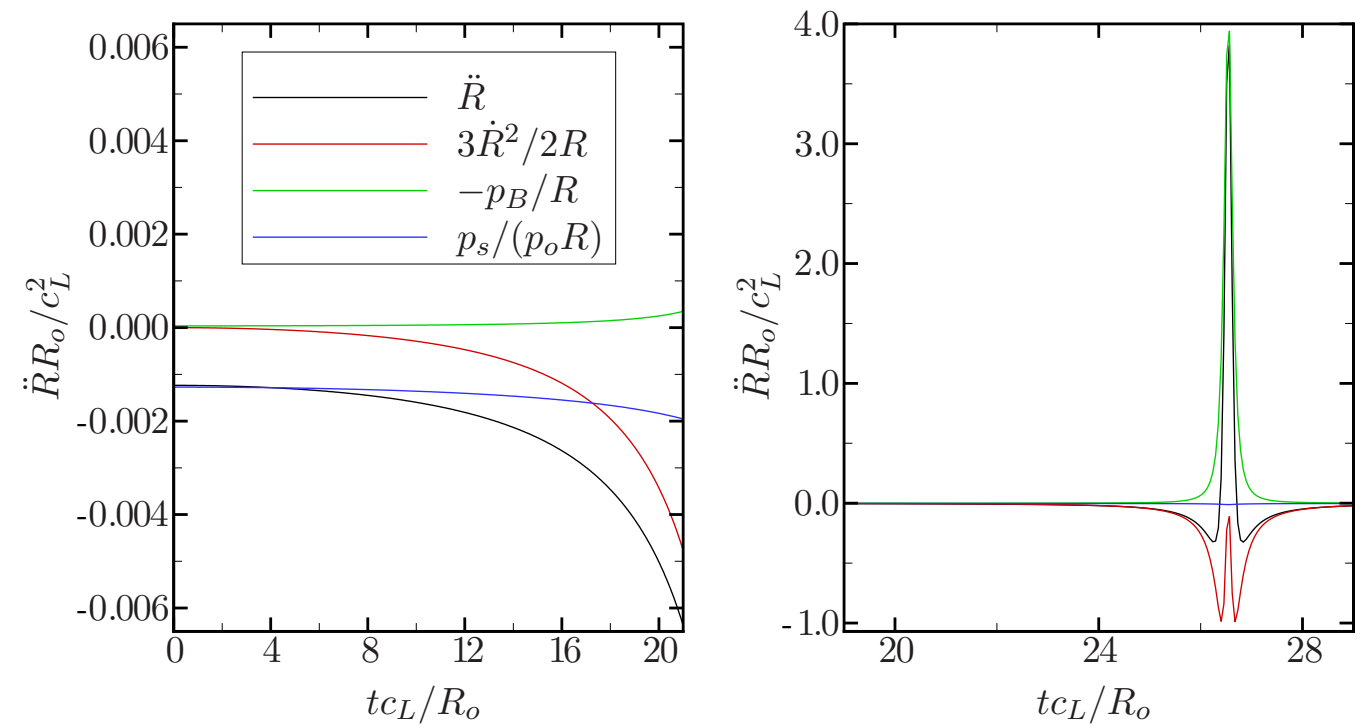

Figure 4.2: Acceleration of the bubble interface for Rayleigh collapse $\left(p_{s} / p_{o}=34\right)$. Left: early time; right: collapse. Note that the vertical scales are different.

Figure 4.2 shows that the the velocity increases according to two regimes during 
collapse. Initially, while $R(t) \approx R_{o}(1-\epsilon)$, where $\epsilon$ is small, the velocity obeys the following slow asymptotic growth:

$$
\dot{R} \rightarrow-\left[2 \frac{p_{\infty}-p_{G o}}{\rho_{L}}\left(1-\frac{R}{R_{o}}\right)\right]^{1 / 2}+O\left(\epsilon^{2}\right)
$$

On the other hand, for $R \ll R_{o}$, the velocity asymptotes to the following fast growth (Brennen, 1995):

$$
\dot{R} \rightarrow-\left(\frac{R_{o}}{R}\right)^{3 / 2}\left[\frac{2 p_{\infty}}{3 \rho_{L}}-\frac{2 p_{G o}}{3(\gamma-1) \rho_{L}}\left(\frac{R_{o}}{R}\right)^{3(\gamma-1)}\right]^{1 / 2}
$$

Just before the rebound, the last term of Equation 4.10 becomes large and must be taken into account. Figure 4.3 shows a phase diagram for the velocity and the radius. Initially, when $R(t) \sim R_{o}$, the velocity follows the slow regime and then transitions smoothly to the fast regime until the deceleration caused by the high gas compression.

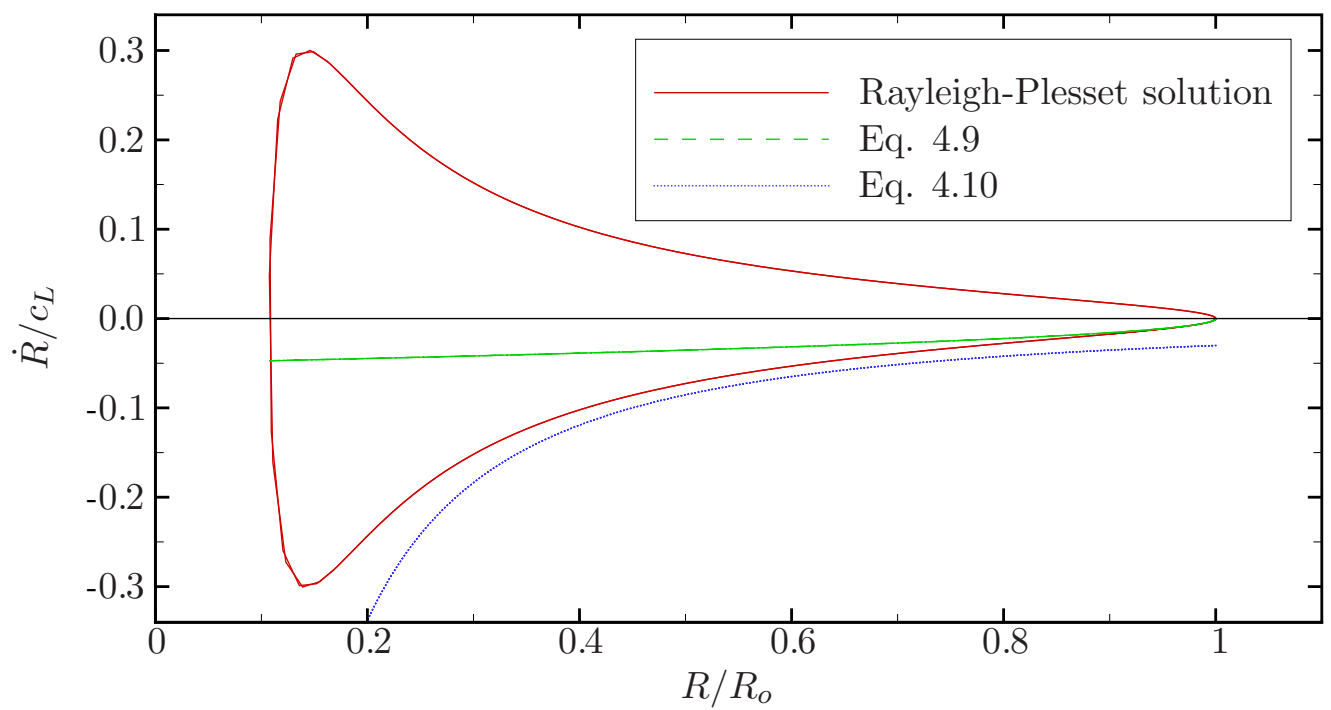

Figure 4.3: Phase diagram for a bubble cycle in Rayleigh collapse $\left(p_{s} / p_{o}=34\right)$. 


\subsubsection{Velocity and pressure fields in the liquid}

The velocity and pressure distributions in the liquid depend on the velocity of the bubble interface and the bubble pressure. The continuity equation provides an expression for the velocity field within the liquid: the velocity is $\dot{R}$ at the bubble interface and decreases as $1 / r^{2}$ with distance.

Equation 4.1 can be integrated from infinity to any point in the liquid to yield

$$
\frac{p(r)-p_{\infty}}{\rho_{L}}=\left(\ddot{R} R+2 \dot{R}^{2}\right) \frac{R}{r}-\frac{1}{2} \dot{R}^{2} \frac{R^{4}}{r^{4}},
$$

where, using Equation 4.4,

$$
\ddot{R} R+2 \dot{R}^{2}=\frac{\dot{R}^{2}}{2}+\frac{p_{B}-p_{\infty}}{\rho_{L}},
$$

and $p_{B}=p_{G o}\left(R_{o} / R\right)^{3 \gamma}$. The pressure at the bubble wall is $p_{B}$ and dominates Equation 4.11 in the last stages of collapse, while the pressure tends to $p_{\infty}$ far away from the bubble. In the region between, the terms on the right-hand side of Equation 4.11 compete, so it is not immediately clear how the pressure distribution behaves. To better understand this, Figure 4.4 shows the pressure as a function of distance at different times for Rayleigh collapse with $p_{s} / p_{o}=34$.

At early times, the pressure monotonically increases from the low initial bubble pressure to $p_{\infty}$. As the bubble collapses, the velocity of the bubble interface and the bubble pressure increase, so that the liquid pressure rises. Between time $t=19.1$ and $t=22.9$ in Figure 4.4, the pressure in the vicinity of the bubble increases beyond $p_{\infty}$, even though $p_{B}<p_{\infty}$, to reach a maximum near the bubble, and then decreases as $1 / r$ with distance. The bubble radius at this maximum, $r^{*}$, is calculated by substituting Equation 4.11 into Equation 4.1:

$$
r^{*}=\frac{R}{\left(\frac{\ddot{R} R}{2 \dot{R}^{2}}+1\right)^{1 / 3}} .
$$

Clearly, this maximum exists only when $\ddot{R} R+2 \dot{R}^{2}>0$, or $\rho_{L} \frac{\dot{R}^{2}}{2}+p_{B}>p_{\infty}$. The 


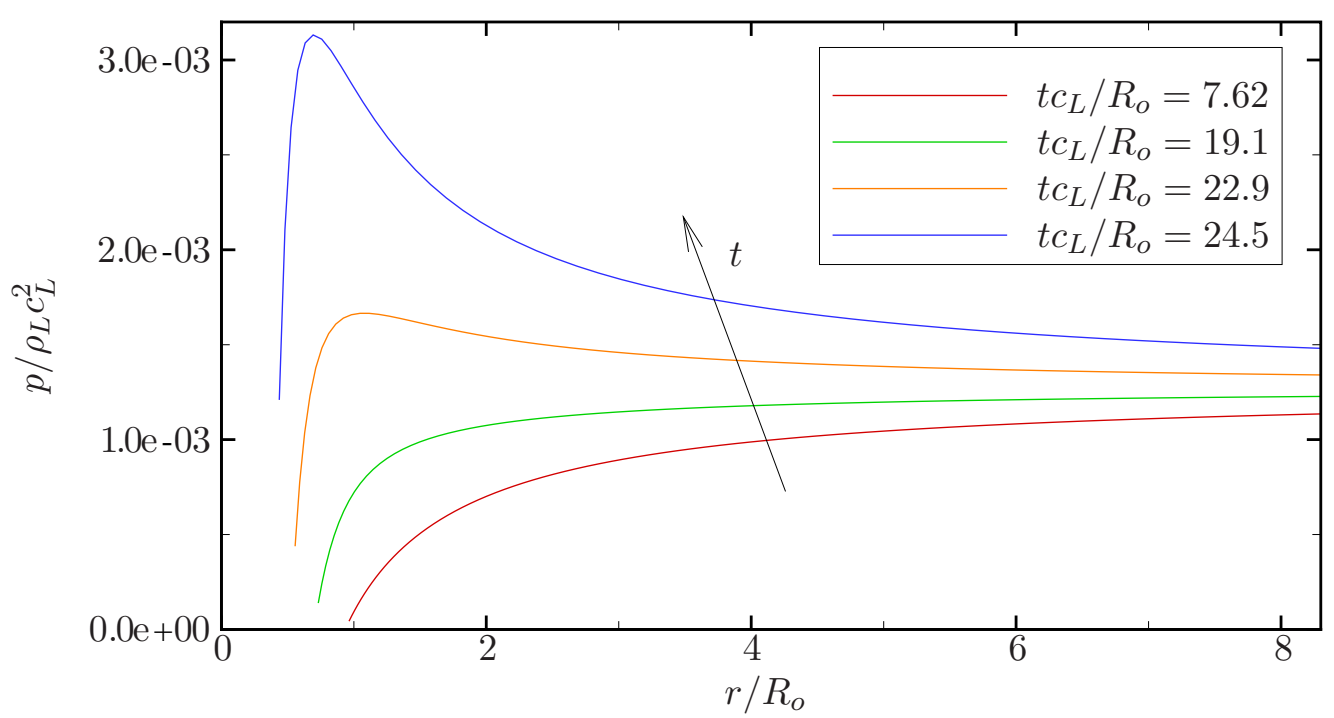

Figure 4.4: Pressure in the liquid as a function of distance for different times for Rayleigh collapse $\left(p_{s} / p_{o}=34\right)$.

value of the pressure maximum is then given by:

$$
\frac{p\left(r^{*}\right)-p_{\infty}}{\rho_{L}}=\frac{3}{2} \dot{R}^{2}\left(\frac{\ddot{R} R}{2 \dot{R}^{2}}+1\right)
$$

In summary, the pressure distribution in the liquid initially decreases monotonically from infinity to that of the bubble. Then, when $R(t)$ reaches a critical value given by Equation 4.13, the velocity of the bubble wall and the bubble pressure both achieve a high enough value that a bifurcation occurs and the pressure has a maximum between infinity and $R$. This maximum moves towards the bubble interface during collapse as the bubble wall speed increases, so that the maximum pressure in the liquid over all time occurs at $r=R$ at collapse. This analysis is verified by considering the Rayleigh collapse problem $\left(p_{s} / p_{o}=34\right)$ shown in Figure 4.4. The pressure distribution is shown in Figure 4.5 for time $t=21.0$ and $t=21.1$. Note that the scale has been adjusted for visualization purposes.

From the solution to the Rayleigh-Plesset equation, the first instance where $r^{*}$ exists is at time, $t=21.067\left(r^{*}=4.51\right)$. Then, it is observed that, for the 


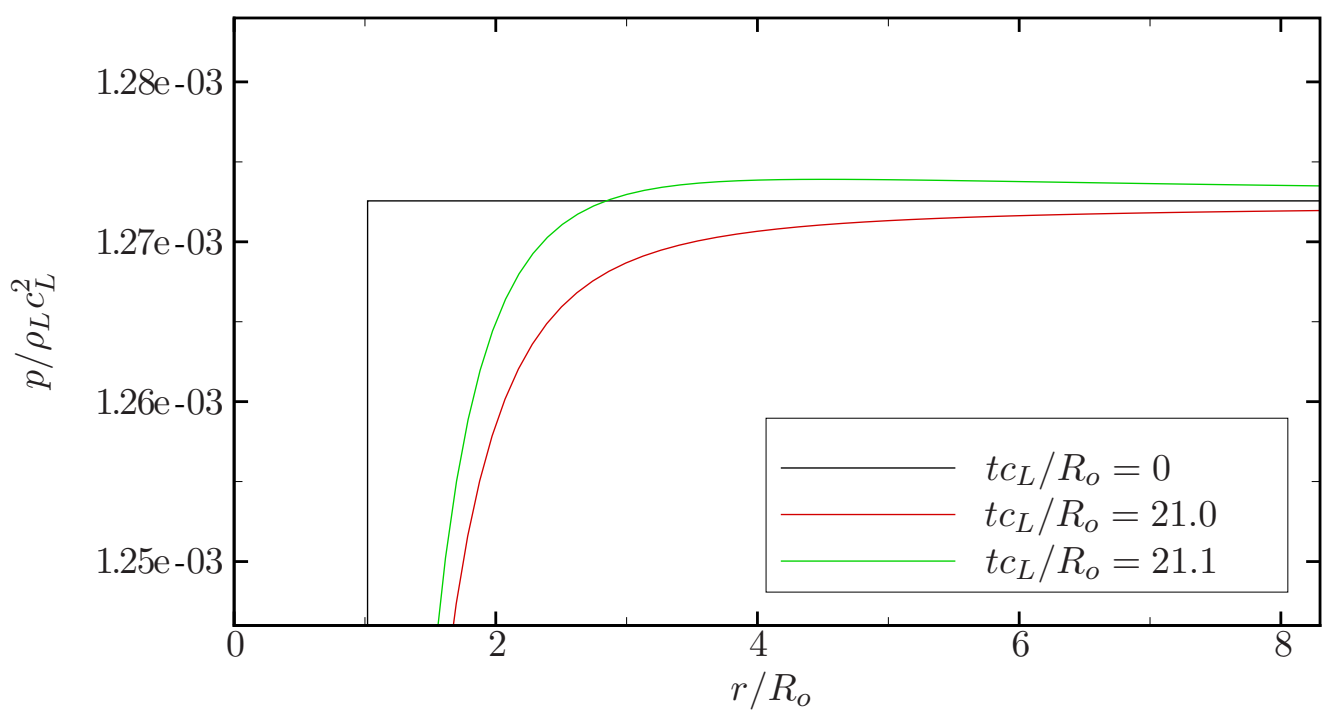

Figure 4.5: Pressure in the liquid as a function of distance for different times to highlight the bifurcation.

range of $r$ considered in the solution to the Rayleigh-Plesset equation, the pressure distribution in the liquid changes from being less than $p_{\infty}$ everywhere to having a maximum near the bubble during the time interval, $21.0-21.1$, as predicted by the theory. It is important to realize that this high local pressure near the bubble is not a cause, but a consequence of the acceleration of the bubble wall. This follows from the fact that Equation 4.4 is only a function of the bubble variables and the pressure at infinity, and does not depend on the details of the flow field in between.

Equation 4.11 was derived by Rayleigh (1917), albeit in a different form, but Benjamin (1958) was the first to understand its practical implications when considering the collapse of a cavity. He postulated that, if the liquid is compressible, the advance of the pressure front would lead to the formation of a shockwave originating in the liquid and propagating into the bubble. This phenomenon is further studied in the numerical simulations (Section 4.5.1). The high local pressure in the liquid is also observed when liquid compressibility is included into the model (Hickling \& Plesset, 1964; Fujikawa \& Akamatsu, 1980). Franc \& Michel 
(2004) considered the case of a bubble containing only vapor and predicted that the maximum to occur in the liquid when $R \approx 0.63 R_{o}$.

\subsubsection{Energy loss by radiation}

Starting from the Rayleigh-Plesset equation 4.4, an expression for the energy balance is derived for the incompressible formulation. Equation 4.7 can be re-arranged in the more enlightening form, for a control volume consisting of the bubble (Cole, 1948):

$$
3\left(\frac{4 \pi}{3} R^{3} \rho_{o}\right) \frac{\dot{R}^{2}}{2}+\left(\frac{4 \pi}{3} R^{3}-\frac{4 \pi}{3} R_{o}^{3}\right) p_{o}-\int_{R_{o}}^{R} p(R) 4 \pi R^{2} d R=0,
$$

where the first term represents the kinetic energy of the radial flow, the second term represents work done against hydrostatic pressure, $p_{o}$, by the liquid, and the third term represents the work done by the (expanding/contracting) bubble against the liquid, which is equivalent to the change in internal energy of the bubble.

The case of a compressible liquid is more complicated. The dilatation is defined as $\lambda=\nabla \cdot \mathbf{u}$. Noting that

$$
\begin{aligned}
{\left[r \frac{\partial u}{\partial t}\right]_{R}^{\infty} } & =\left[\frac{1}{r} \frac{\partial}{\partial t}\left(r^{2} u\right)\right]_{R}^{\infty}=\int_{R}^{\infty}\left(-\frac{1}{r^{2}}\right) \frac{\partial}{\partial t}\left(r^{2} u\right) d r+\int_{R}^{\infty} \frac{1}{r} \frac{\partial}{\partial r}\left(\frac{\partial}{\partial t}\left(r^{2} u\right)\right) d r \\
& =-\int_{R}^{\infty} \frac{\partial u}{\partial t} d r+\int_{R}^{\infty} r \frac{\partial \lambda}{\partial t} d r
\end{aligned}
$$

the momentum equation 4.1 can be integrated from $R$ to $\infty$ to yield:

$$
-\left[r \frac{\partial u}{\partial t}\right]_{R}^{\infty}+\int_{R}^{\infty} r \frac{\partial \lambda}{\partial t} d r+\left[\frac{u^{2}}{2}\right]_{R}^{\infty}+\int_{p(R)}^{p_{o}} \frac{d p}{\rho}=0 .
$$

This can be re-arranged as

$$
-\left[r\left(\frac{\partial u}{\partial t}+u \frac{\partial u}{\partial r}\right)\right]_{R}^{\infty}+\left[r u \frac{\partial u}{\partial r}\right]_{R}^{\infty}+\int_{R}^{\infty} r \frac{\partial \lambda}{\partial t} d r+\left[\frac{u^{2}}{2}\right]_{R}^{\infty}+\int_{p(R)}^{p_{o}} \frac{d p}{\rho}=0
$$

Noting that $r u \partial u / \partial r=r u \lambda-2 u^{2}$ and $u(\infty, t)=0$, the following form of the 
compressible Rayleigh-Plesset equation is obtained:

$$
R \ddot{R}+\frac{3}{2} \dot{R}^{2}-R \dot{R} \lambda(R)+\int_{R}^{\infty} r \frac{\partial \lambda}{\partial t} d r+\int_{p(R)}^{p_{o}} \frac{d p}{\rho}=0
$$

If the stiffened EOS is used, then

$$
\lambda(R)=-\left[\frac{1}{\rho c^{2}} \frac{d p}{d t}\right]_{R}=3 \frac{\gamma p(R)}{\rho c^{2}} \frac{\dot{R}}{R}=3 \frac{p(R)}{p(R)+P_{\infty}} \frac{\dot{R}}{R} .
$$

Thus, the only term that causes difficulty is the integral term for the time rate of change of $\lambda$. In principle, the momentum equation would have to be solved in the liquid with appropriate boundary conditions at the bubble and at infinity to compute $\partial \lambda / \partial t$.

In order to obtain an equation for the balance of energy, the model of Herring (1941) is followed. Although less accurate than that of Gilmore (1952), ${ }^{3}$ the integration term involving $\partial \lambda / \partial t$ is straightforward. For small-amplitude oscillations, $\rho$ and $c$ are constant, and $u$ satisfies the wave equation, so that $\lambda$ does as well (i.e, $\left.\lambda=f\left(r-c_{o} t\right)\right)$. Then,

$$
\int_{R}^{\infty} r \frac{\partial \lambda}{\partial t} d r=-c_{o} \int_{R}^{\infty} \frac{\partial}{\partial r}(r \lambda) d r=c_{o} R \lambda(R)
$$

It is recommended to further add the correction term (Herring, 1941)

$$
-\frac{1}{c_{o}} \frac{1}{R} \frac{d}{d t}\left(R^{2} \dot{R}^{2}\right)=\frac{2}{3} \frac{d}{d R}\left(R^{3} \dot{R}^{3}\right)
$$

to the equation. Thus, the momentum equation 4.19 becomes:

$$
R \ddot{R}+\frac{3}{2} \dot{R}^{2}+\frac{p_{o}-p(R)}{\rho_{o}}=\frac{2}{3} \frac{1}{c_{o}} \frac{d}{d R}\left(R^{3} \dot{R}^{3}\right)+\frac{R}{\rho_{o} c_{o}}\left[\frac{d p}{d t}\right]_{R}\left(1-\frac{\dot{R}}{c_{o}}\right) .
$$

\footnotetext{
${ }^{3}$ The Gilmore equation (Gilmore, 1952) is written as

$$
\left(1-\frac{\dot{R}}{c}\right) R \ddot{R}+\frac{3}{2}\left(1-\frac{1}{3} \frac{\dot{R}}{c}\right) \dot{R}^{2}=\left(1+\frac{\dot{R}}{c}\right) h+\frac{R}{c} \frac{d h}{d t} .
$$
}

where the enthalpy is given by $d h=\frac{d p}{\rho}$. 
Equation 4.23 can then be integrated with respect to $R$ to yield a balance of energy:

$$
\begin{gathered}
3\left(\frac{4 \pi}{3} R^{3} \rho_{o}\right) \frac{\dot{R}^{2}}{2}+\left(\frac{4 \pi}{3} R^{3}-\frac{4 \pi}{3} R_{o}^{3}\right) p_{o}-\int_{R_{o}}^{R} p(R) 4 \pi R^{2} d R \\
=4\left(\frac{4 \pi}{3} R^{3} \rho_{o}\right) \frac{\dot{R}^{2}}{2} \frac{\dot{R}}{c_{o}}+\int_{R_{o}}^{R} \frac{1}{c_{o}}\left[\frac{d p}{d t}\right]_{R}\left(1-\frac{\dot{R}}{c_{o}}\right) 4 \pi R^{3} d R
\end{gathered}
$$

The left-hand side of the equation represents conservation of energy for incompressible motion, i.e., Equation 4.15. Thus, changes in this value due to the right-hand side must be caused by energy radiation due to the compressibility of the liquid. Recalling that $[d p / d t]_{R}=-3 \gamma p(R) \dot{R} / R$, the right-hand side consists in a correction of order $O\left(\dot{R} / c_{o}\right)$ to the incompressible expression. ${ }^{4}$ The first term on the right-hand side is a correction to the kinetic energy and the second term is a correction to the work done by the bubble. Equation 4.24 is used to compute the radiated energy in Section 4.5.3.

\subsubsection{Non-spherical perturbations}

Large positive and negative accelerations occur during bubble collapse and lead to the catastrophical growth of small perturbations. Birkhoff (1954) and Plesset \& Mitchell (1956) considered the spherical equivalent to the planar Rayleigh-Taylor instability. The bubble radius may be perturbed using Lagrange polynomials (axisymmetric spherical harmonics), so that the resulting radius is given by

$$
R_{s}(t)=R(t)+\sum_{n} a_{n}(t) P_{n}(\cos \theta)
$$

where $\left|a_{n}\right| \ll R$. The Rayleigh equation 4.4 still holds, but the amplitude of each mode is governed by the following ordinary differential equation:

$$
\ddot{a}_{n}+3 \frac{\dot{R}}{R} \dot{a}_{n}-(n-1) \frac{\ddot{R}}{R} a_{n}=0
$$

\footnotetext{
${ }^{4}$ Cole (1948) suggests to discard terms in $\dot{R} / c_{o}$. However, this leads to the cancellation of the entire right-hand side, so that the incompressible energy balance is recovered.
} 
This second-order ordinary differential equation can be integrated simultaneously with the Rayleigh-Plesset using appropriate initial conditions for $a_{n}(0)$ and $\dot{a}_{n}(0)$. During collapse, $\dot{R}<0$, while $\ddot{R} \geq 0$ until just before the rebound. This analysis is used to understand the jet formation mechanism in Section 4.4.

\subsection{Flow visualization}

In order to understand the general flow features of non-spherical bubble collapse, numerical Schlieren and pressure contours in the $x r$-plane are shown. The calculation of the numerical Schlieren fields, which is based on the density gradient and is discussed further in Sections 3.8.2 and C.5, provides means to visualize both interfaces and shocks. The finest grid spacing for all cases is $\Delta x_{\min }=6.67 \times 10^{-3}$, except for Rayleigh collapse near a wall under cavitation conditions, where $\Delta x_{\min }=$ $10^{-2}$. This is due to the fact that a larger domain is required because of the slow process, in order to prevent reflections from the boundaries, as discussed in Section 3.5. Two types of collapse are considered: Rayleigh collapse under cavitation conditions $\left(p_{s} / p_{o} \approx 34\right)$ and shock-induced collapse of a pre-existing nucleus or bubble $\left(p_{s} / p_{o}=353\right)$, both in a free field and near a wall.

\subsubsection{Free-field collapse}

\section{Free-field Rayleigh collapse with $p / p_{o}=34$}

First, Rayleigh collapse driven by an initial pressure ratio of $p_{s} / p_{o}=34$ in a freefield is considered. Figure 4.6 shows pressure and numerical Schlieren contours, at $t c_{L} / R_{o}=0.87,13.1,22.6,24.4,26.1,29.0$.

As discussed in Section 4.3.2, the initial conditions for Rayleigh collapse constitute a Riemann problem along the interface. In frame 1, the expansion wave is propagating radially outwards, while the corresponding shockwave is converging within the bubble. ${ }^{5}$ The shock has converged at the center of the bubble in frame 2 , though this is barely visible in the figure. The bubble then collapses, while the

\footnotetext{
${ }^{5}$ The shock amplitude is too small to be noticed on the plots.
} 


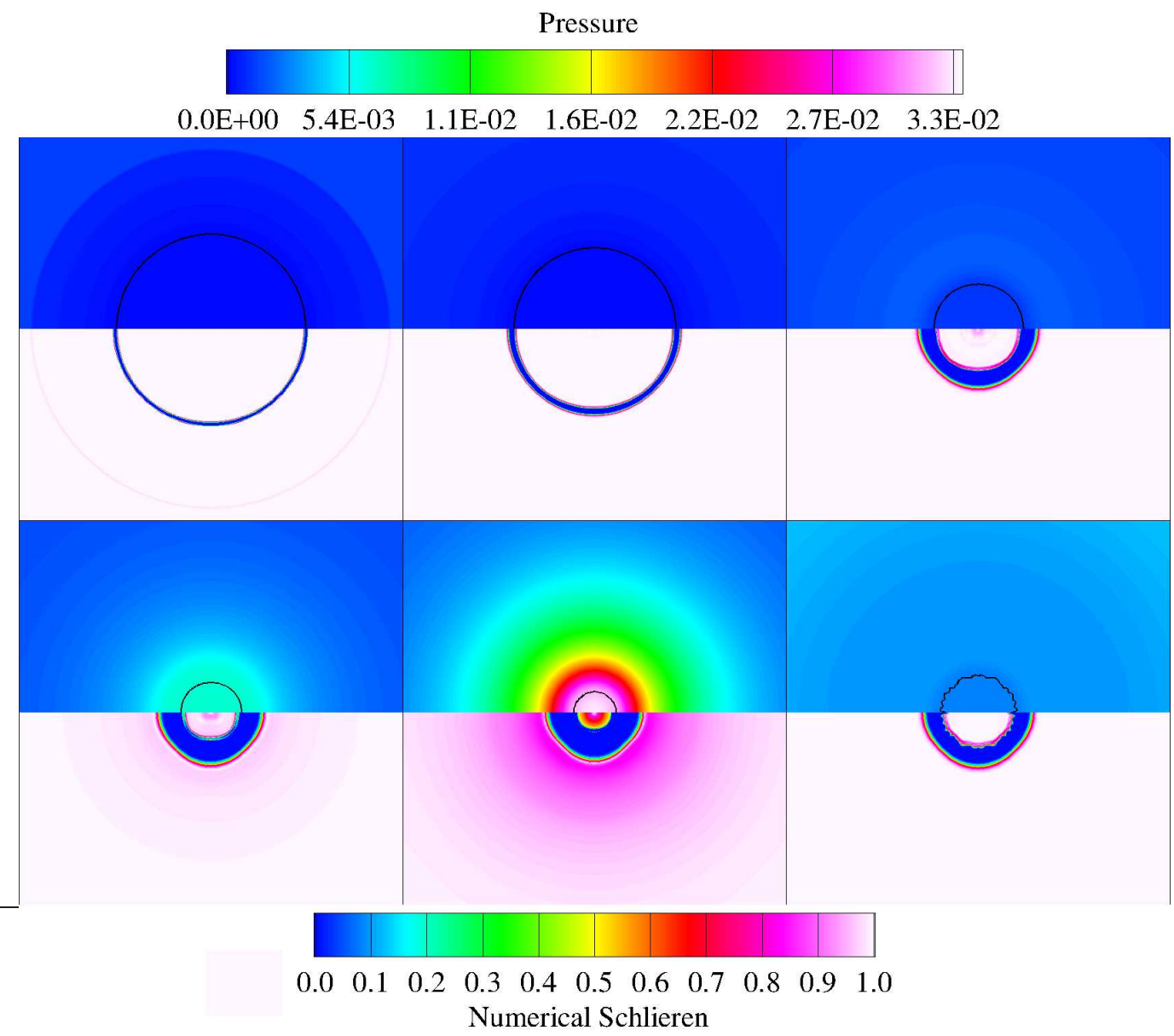

Figure 4.6: Pressure (top) and numerical Schlieren (bottom) contours for Rayleigh collapse in a free field $\left(p_{s} / p_{o}=34\right)$ at $t c_{L} / R_{o}=0.87,13.1,22.6,24.4,26.1,29.0$.

shock propagates radially outwards, eventually hitting the interface and reflecting almost in its entirety due to the large impedance mismatch. This effect is minor and does not lead to significant instabilities, such as Richtmyer-Meshkov. Because the bubble is contracting, the internal shock strengthens and converges again at the center of the bubble in frame 3 . The flow is rushing to fill the void left by the collapsing bubble, as illustrated by the radial variations in the pressure field. The bubble starts to compress significantly in frame 4, as the shockwave propagates within the bubble. As expected from the analysis of Section 4.1.2, a region of high pressure is visible in the liquid just outside the bubble. The minimum volume and 
consequently maximum bubble pressure are achieved in frame 5; pressure waves are radiated away from the bubble. As the bubble rebounds, the interface becomes distorted, as illustrated in frame 6. This results from instabilities in the last stages of collapse where the acceleration changes sign (Brennen, 2002) and is discussed further in Section 4.3.3.

The case of Rayleigh collapse driven by an initial pressure ratio of $p_{s} / p_{o}=353$ is similar. Because of the larger pressure ratio, the initial expansion wave and the shockwave propagating within the bubble are sharper, the collapse time is shorter, the pressure pulse released upon collapse is stronger, and the interfacial instabilities are enhanced.

\section{Free-field shock-induced collapse with $p / p_{o}=353$}

The collapse of a bubble due to the passage of a shock with a pressure ratio of $p_{s} / p_{o}=353$ is considered. Figure 4.7 shows pressure and numerical Schlieren contours, at $t c_{L} / R_{o}=1.65,5.13,7.19,7.93,8.70,9.70$.

When the left-moving shock hits the bubble, an expansion wave is reflected because of the impedance mismatch between water and air, while a weak shockwave is transmitted into the bubble in frame 1. After reaching the top of the bubble, the external shock diffracts until it intersects the $r=0$ axis; the shock along $r=0$ thus lags the main front. Meanwhile, the internal shock focuses within the bubble over a wider area, but not at the center, as seen in frame 2 . The proximal bubble end flattens as it accelerates, while the internal shock reflects within the bubble in frame 3. Eventually, the proximal end involutes, so that the re-entrant jet is manifest in frame 4. A region of high pressure is visible behind the jet, where the interface velocity is the highest, similarly to the free-field Rayleigh collapse. The jet impacts the distal side in frame 5 and generates a large water-hammer pressure; a precursor shock is emitted prior to this event. The shock emission is carefully examined in Section 4.5.1. While the water-hammer pressure propagates radially outwards in frame 6 , the bubble takes the form of a vortex ring and convects downstream. 


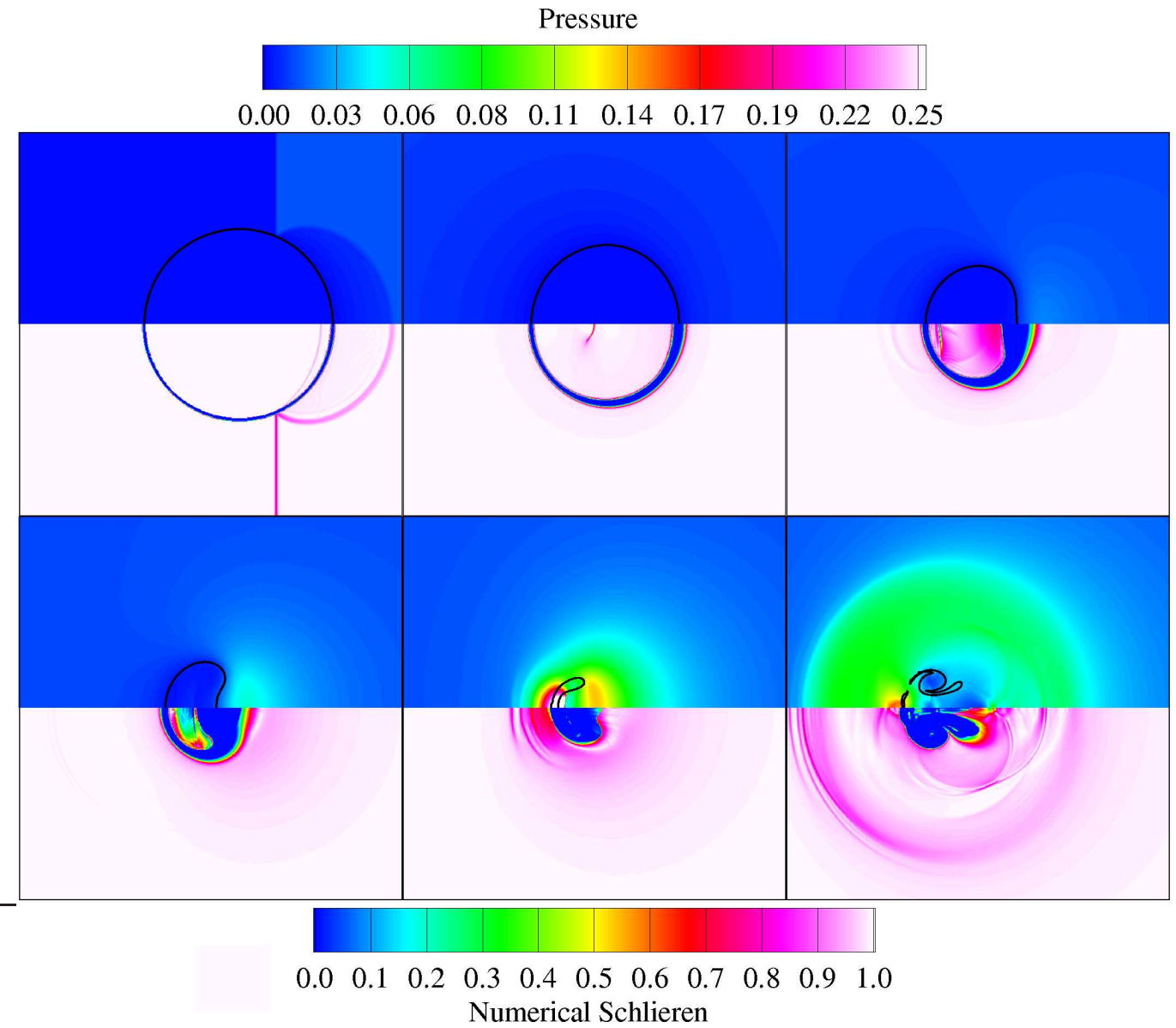

Figure 4.7: Pressure (top) and numerical Schlieren (bottom) contours for shockinduced collapse in a free field $\left(p_{s} / p_{o}=353\right)$ at $t c_{L} / R_{o}=1.65,5.13,7.19,7.93$, 8.70, 9.70 .

\subsubsection{Collapse near a wall}

\section{Rayleigh collapse near a wall with $p_{s} / p_{o}=34$}

First, Rayleigh collapse driven by an initial pressure ratio of $p_{s} / p_{o}=34$ near a wall $\left(H_{o} / R_{O}=1.5\right)$ is considered. Figure 4.8 shows pressure and numerical Schlieren contours at $t c_{L} / R_{o}=1.16,26.1,28.1,29.3,30.4,32.7$. The wall is denoted by the grey region on the left of the domain.

In frame 1, the initial expansion is propagating radially outwards and reflects off the wall back onto the bubble, while the shock converges within the bubble. The 


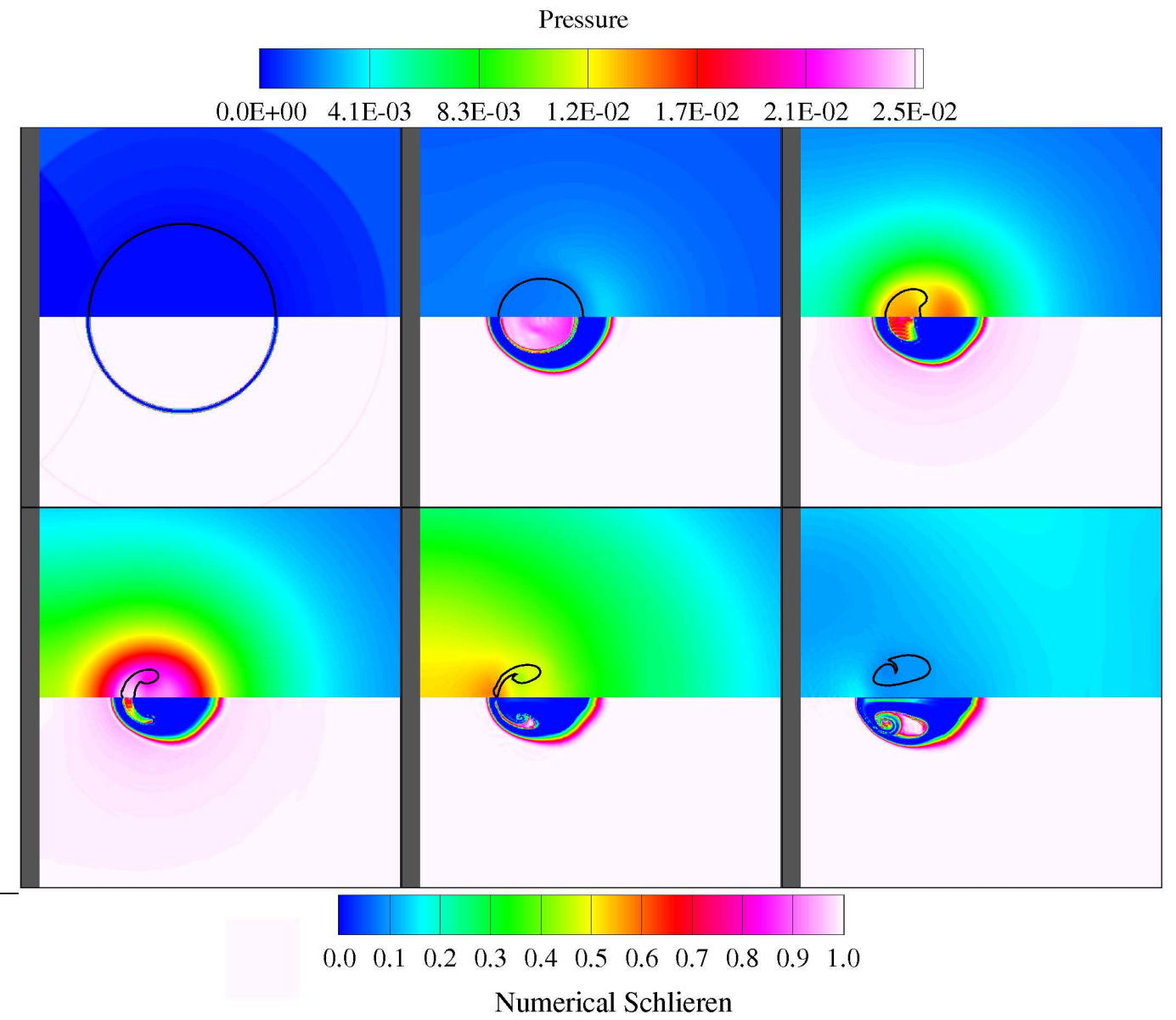

Figure 4.8: Pressure (top) and numerical Schlieren (bottom) contours for Rayleigh collapse near a wall $\left(p_{s} / p_{o}=34, H_{o} / R_{o}=1.5\right)$ at $t c_{L} / R_{o}=1.16,26.1,28.1,29.3$, $30.4,32.7$.

presence of the wall is communicated to the bubble via the reflection of the initial expansion. As a result, the pressure decreases locally along the bubble interface after the passage of the expansion. Because the strength of the expansion decreases with distance, it does not affect the proximal side as much. During the collapse, the bubble migrates towards the wall. The proximal side flattens in the direction normal to the wall (Rattray, 1951; Plesset \& Chapman, 1971), as shown in frame 2. These features result from the presence of the wall, as the flow of liquid attempting to fill the void left by the collapsing bubble is retarded. A region of high pressure 
forms in the liquid behind the proximal end (Blake et al., 1986; Best \& Kucera, 1992), after that end involutes. In frame 3, the air within the bubble is already highly compressed as the re-entrant jet directed towards the wall forms. Upon reaching its minimum volume in frame 4, the kidney-shaped bubble generates a pressure pulse propagating radially outwards, though it is stronger in the direction of the jet. This pulse hits the wall in frame 5. In the meantime, the bubble has taken the shape of a vortex ring that is convecting towards the wall as it expands and collapses, as observed in frame 6. The interface appears to have broken up upon the impact of the jet; this feature is caused by the dissipation of the numerical scheme and the finite grid size.

Again, the flow features of a Rayleigh collapse driven by an initial pressure ratio of $p_{s} / p_{o}=714$ are similar. As in the free-field case, a more violent collapse occurs because of the larger pressure ratio.

\section{Shock-induced collapse near a wall with $p_{s} / p_{o}=353$}

The shock-induced collapse of a bubble near a wall $\left(p_{s} / p_{o}=353, H_{o} / R_{o}=1.5\right)$ is considered. Figure 4.9 shows pressure and numerical Schlieren contours, at $t c_{L} / R_{o}=1.01,4.56,7.00,7.90,8.60,9.80$. The wall is denoted by the grey region on the left of the domain.

The collapse is characterized by a combination of the effects observed in freefield shock-induced collapse and Rayleigh collapse near a wall. The left-propagating incoming shock reflects off the bubble as an expansion wave, while a weak shock is transmitted into the bubble (frame 1). Then, the external shock diffracts around the bubble and reflects off the wall; the shock at $r=0$ lags the main front because of the diffraction. In frame 2, the external shock passes over the bubble again, while the initial internal shock focuses; a second shock is transmitted within the bubble, from the left this time, because of the interaction of the external shock reflected off the wall with the distal side of the bubble. Although not visible in the plots, waves are reflected between the bubble and the wall. The far end of the bubble flattens in frame 3; the internal shock structure is highly complex. As noted 


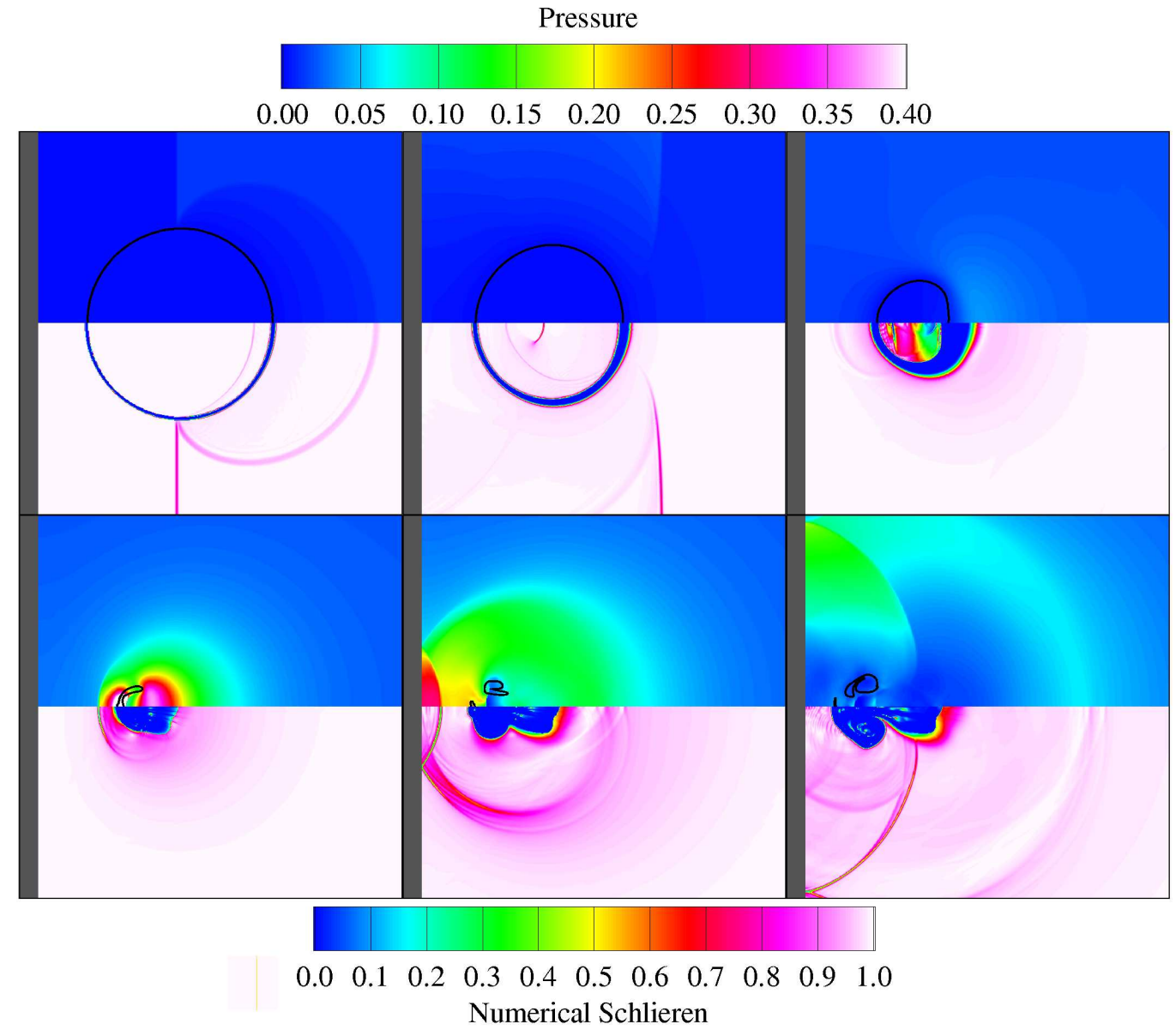

Figure 4.9: Pressure (top) and numerical Schlieren (bottom) contours for shockinduced collapse near a wall $\left(p_{s} / p_{o}=353, H_{o} / R_{o}=1.5\right)$ at $t c_{L} / R_{o}=1.01,4.56$, $7.00,7.90,8.60,9.80$.

previously, a region of high pressure behind the jet is observed in frame 4 . The re-entrant jet hits the distal side in frame 5 , after emitting a precursor shockwave. The water-hammer pressure generated in the liquid reflects off the wall in frame 6 and interacts with the rebounding bubble, which has now taken the form of a vortex ring. Upon reflection off the bubble, the amplitude of the pressure wave inverts because of the impedance mismatch, so that a tensile wave is reflected back to the wall. 


\subsection{Dynamics of collapse}

In the present section, the dynamics of non-spherical collapse are characterized and certain properties of the re-entrant jet are studied.

\subsubsection{Time scales in bubble collapse}

In order to illustrate the chronology of the events that occur during bubble collapse, Figure 4.10 shows a schematic of the different important phenomena that occur during free-field shock-induced collapse for $p_{s} / p_{o}=353$. The events of interest are listed in Table 4.1 and Figure 4.11 shows the time at which each of these events occurs as a function of the initial pressure ratio across the shock. In the case of Rayleigh collapse near a wall, $t_{d i f}$ is irrelevant, but $t_{\text {shock }}$ still occurs because of the shock released due to the initial set-up, as explained in Section 4.3.2.

Table 4.1: Events of interest during bubble collapse.

\begin{tabular}{l|l} 
Symbol & Description \\
\hline$t_{d i f}$ & The external shock has diffracted about the bubble. \\
$t_{\text {shock }}$ & The internal shock has traversed the bubble. \\
$t_{v_{j e t}}$ & The maximum jet velocity is achieved. \\
$t_{c o n}$ & The bubble shape becomes concave. \\
$t_{\dot{\Gamma}_{\max }}$ & The rate of change of circulation achieves its maximum. \\
$t_{\Gamma_{\max }}$ & The circulation achieves its maximum. \\
$t_{\ddot{R}}$ & The gross interface acceleration changes sign. \\
$\tau_{c}$ & The bubble achieves its minimum radius (collapse time).
\end{tabular}

The time at which the external shock has diffracted over the bubble is essentially constant, because the shock speed does not vary much for the range of pressure ratios considered $\left(p_{s} / p_{o}=34-714\right)$. The time it takes for the internal shock to traverse the bubble depends on the transmission of the incoming shock 


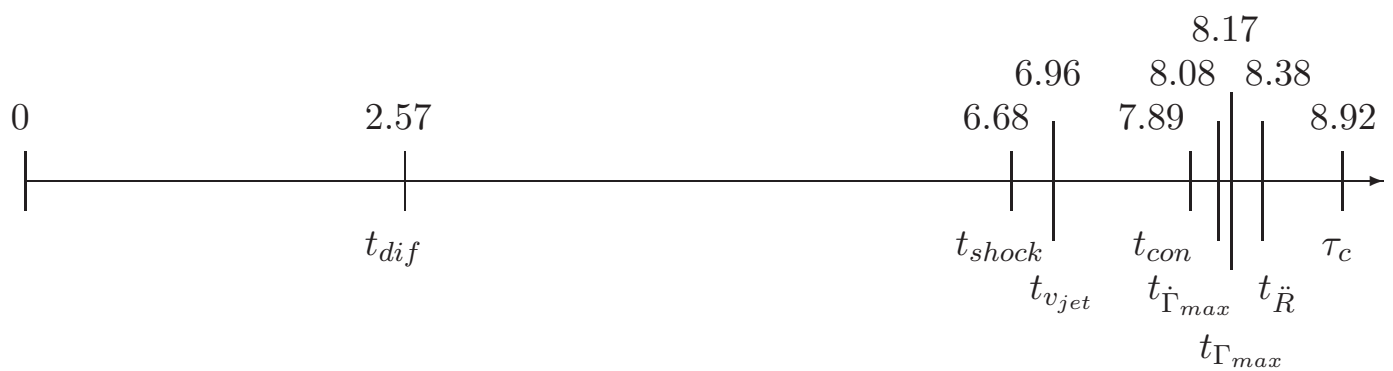

Figure 4.10: Chronology of events in free-field shock-induced collapse $\left(p_{s} / p_{o}=\right.$ $353)$.

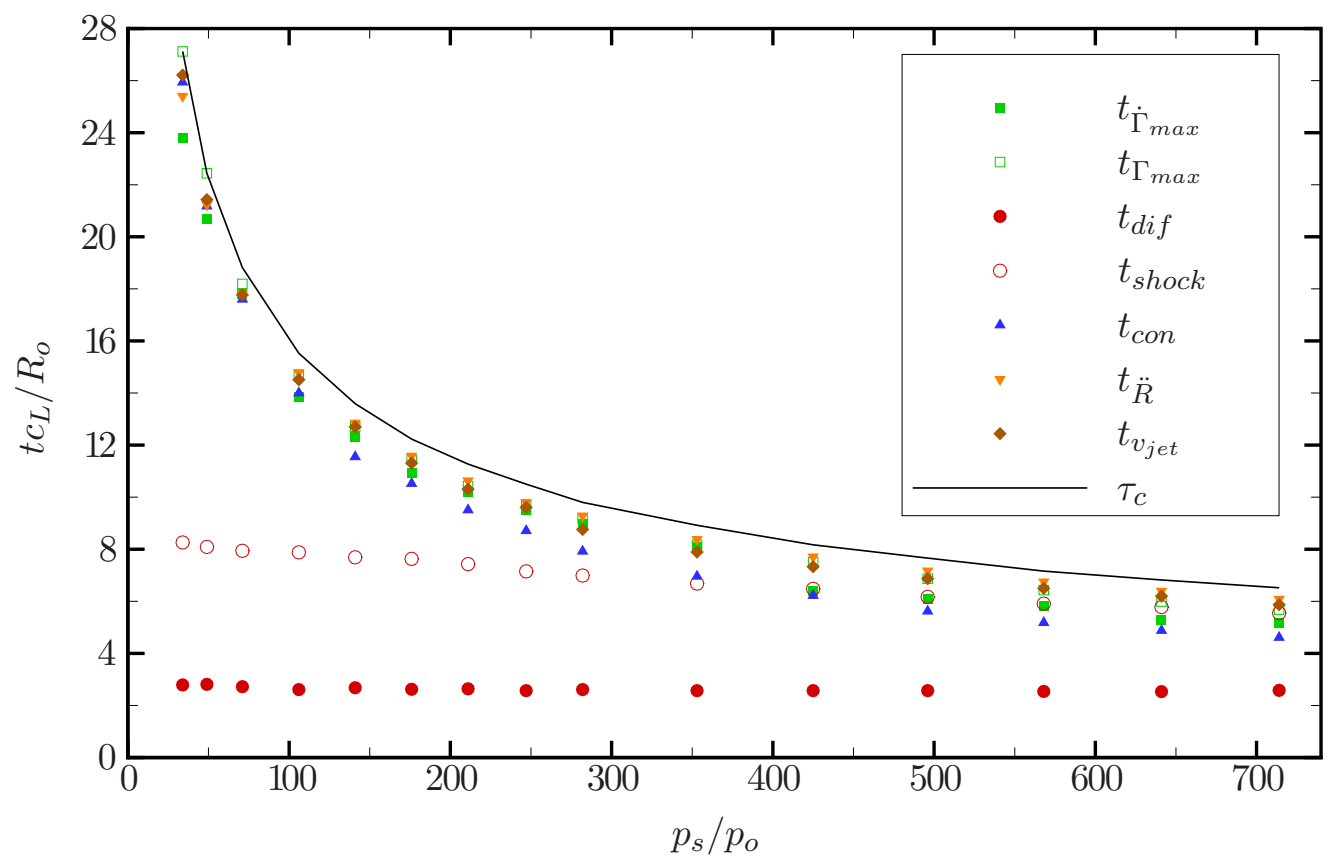

Figure 4.11: Chronology of events as a function of the pressure ratio across the shock for free-field shock-induced collapse.

and decreases essentially linearly with the pressure ratio across the shock, as expected from the analysis of Section 4.3.2. These shock-propagation phenomena are not influenced by the bubble collapse.

On the other hand, the other quantities follow a $1 / \sqrt{p_{s} / p_{o}}$ behavior characteristic of the collapse time described in Section 4.3.7. In the case of low pressure ratios, the time at which the maximum circulation is achieved is almost identical 
to the collapse time, while the time at which the internal shock has traversed the bubble is much shorter. However, as the pressure ratio is increased, the collapse is so rapid that the jet almost catches up with the internal shock. Another interesting observation is that, except for the very shock pressure ratios, the bubble becomes concave just before the rate of change of circulation reaches its maximum, thus suggesting a link between the two mechanisms.

In the range of parameters considered in the present study, the generation of the water-hammer pressure is essentially simultaneous with the time when the minimum volume is achieved. Thus, the wave merge into a single front (Sankin et al., 2005), so that the two events are undistinguishable at a certain distance from the collapse point.

\subsubsection{Initial motion of the interface}

In Rayleigh collapse, the problem is started by instantaneously raising the liquid pressure (or decreasing the bubble pressure). In the present compressible formulation, this corresponds to a gas/liquid Riemann problem across the interface. In the limit of small time, this problem reduces approximately to the traditional one-dimensional planar problem. Similarly, when the shockwave intersects the bubble in shock-induced collapse, the conditions along $r=0$ are those of a Riemann problem across the interface; for interface points off-axis, the problem is fully multi-dimensional. As a result of such initial conditions, an expansion wave is released into the liquid, while a shockwave propagates radially inwards in the bubble, as observed in Section 4.2. This discontinuous feature of the problem for Rayleigh collapse is introduced because the bubble growth is neglected and appropriate initial conditions must be specified. When considering the compressible Rayleigh-Plesset equation, Plesset (1969) used the following initial condition:

$$
\dot{R}_{o} \approx \int_{0}^{h} \frac{d h}{c} \approx \frac{p\left(R_{o}\right)-p_{o}}{\rho_{L} c_{L}}
$$


This expression is derived by re-arranging the Gilmore equation and retaining the terms relevant for the initial conditions; it is in fact a linearization of Equation A.24 in Appendix A, which is the normal shock relation for velocity. In the case of shock-induced collapse, the initial velocity can be estimated using linearized shock relations (Thompson, 1984),

$$
u_{C D} \approx 2 u_{p} \approx 2 \frac{p_{s}-p_{o}}{\rho_{L} c_{L}}
$$

where $u_{p}$ is the particle velocity behind the incoming shock. This expression is valid for the interaction of a shock and an interface between two fluids with very different acoustic impedances and where the fluid in which the shock is propagating has the higher impedance.

Figure 4.12 shows the initial velocity of the interface, $u_{C D}$, as a function of the initial pressure ratio across the interface for Rayleigh collapse and shock-induced collapse. Results from the simulations and from the corresponding one-dimensional Riemann problems are included, as well as the linearized approximations given by 4.27 and 4.28. The velocities are negative because the shock is propagating from right to left, and the position of the interface is calculated as described by Equation C.12 of Appendix C after one time-step.

The agreement between the simulation and the exact solution to the onedimensional Riemann problem is good, though there are deviations in the shockinduced collapse results for large pressure ratio. Small differences stem from the approximation to determine the jet position and from the fact that the data is only generated at a finite time. For a given pressure ratio, the interface velocity in the shock-induced collapse is twice the particle speed behind the shock, as expected from Equations 4.27 and 4.28.

The strength of the transmitted shock along $r=0$ just after refraction can be evaluated by solving the one-dimensional Riemann problem. Then, the Mach number of the transmitted shock can be expressed using Equation A.25, with $P_{\infty}=0$ in the bubble. Figure 4.13 shows the pressure ratio and the Mach number 


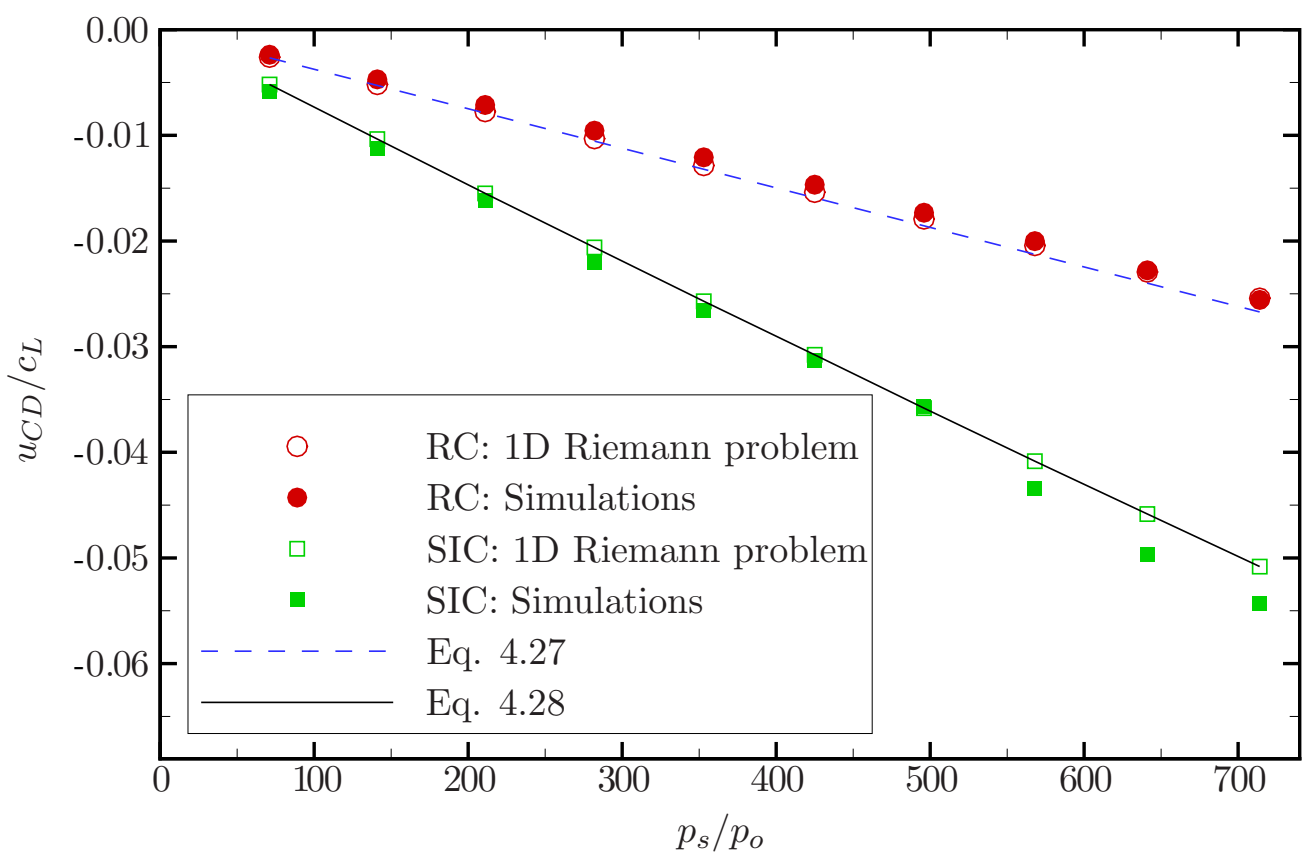

Figure 4.12: Initial velocity of the interface for Rayleigh collapse and shock-induced collapse for the simulations and different theoretical models.

of the transmitted shock as a function of the pressure ratio and Mach number of the incoming shock in water. Given that the pressure ratio across the transmitted shock in Rayleigh collapse is $1+\alpha x$, where $x$ is the initial pressure ratio across the interface, the pressure ratio across the transmitted shock is approximately $1+2 \alpha x$, as expected from the linearized shock relations (e.g., Equation 4.28).

Figures 4.12 and 4.13 illustrate that the initial motion of the bubble is well captured. As the bubble collapses, the behavior deviates from that of the onedimensional problem and focusing takes place, so that the velocity of the interface and shockwave increases. As a result of the focusing, the strength of the shock propagating within the bubble (i.e., the transmitted shockwave, in shock-induced collapse) increases. 

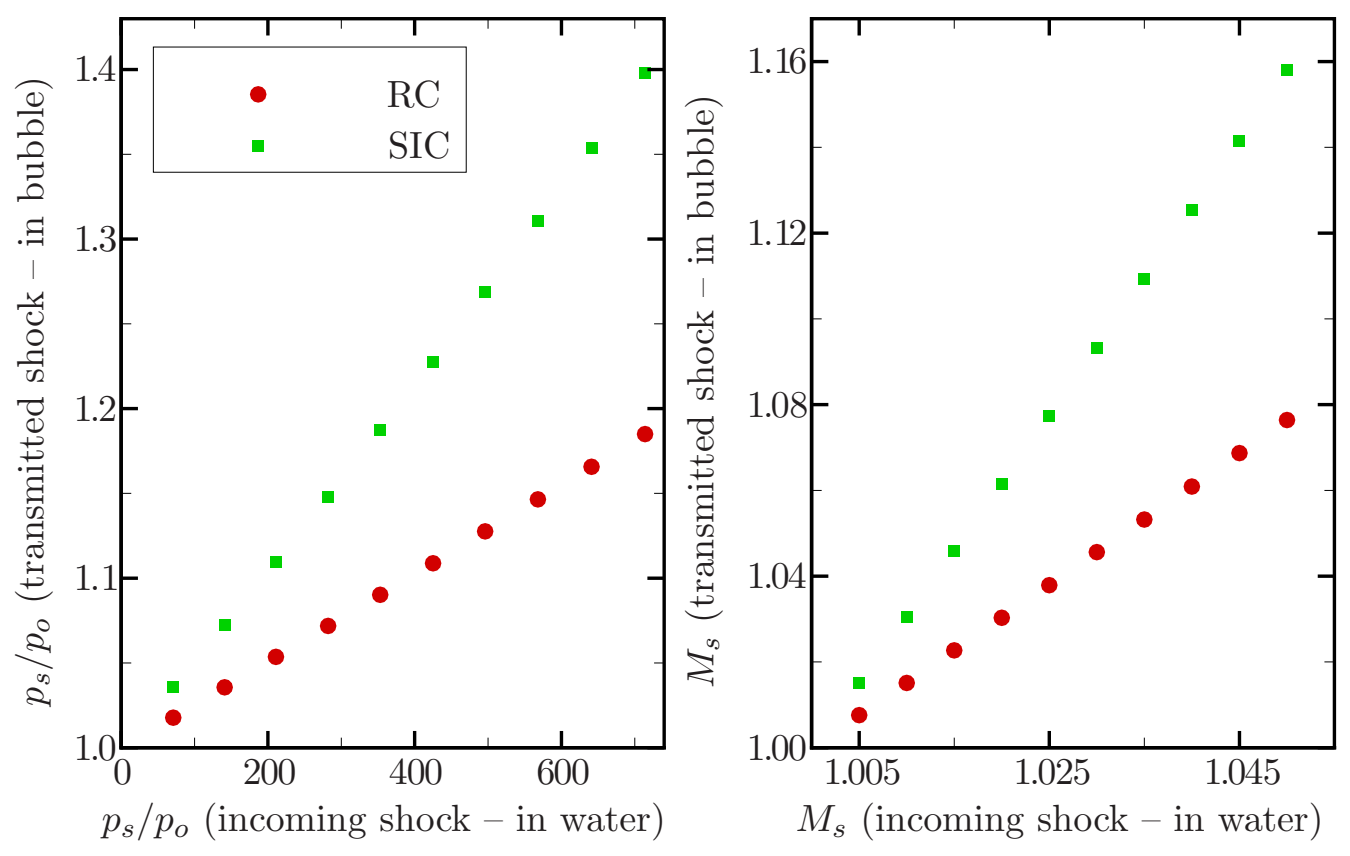

Figure 4.13: Pressure ratio (left) and Mach number (right) of the transmitted shock for Rayleigh collapse and shock-induced collapse.

\subsubsection{Interfacial instabilities}

Because of the large accelerations occurring during bubble collapse, the interface is prone to small-scale perturbations that grow catastrophically in a finite time. In particular, Rayleigh-Taylor, Richtmyer-Meshkov, and Kelvin-Helmholtz instabilities are relevant in the bubble collapse problems considered in the present work. In this section, these flow features are considered on a small scale (i.e., shortwavelength perturbations).

As described in Section 4.1.4 for spherical bubbles, the interface is most unstable just before the rebound, when $\dot{R}<0$, while $\ddot{R} \geq 0$. Then, high-frequency perturbations are expected to grow. This effect is illustrated in Figure 4.14, which shows numerical Schlieren contours of free-field Rayleigh collapse with $p_{s} / p_{o}=353$ at $t c_{L} / R_{o}=7.46,7.63$, when $\ddot{R}$ is reaches its largest (positive) value. This occurs late in the collapse, so that the scale is magnified. As noted in Section 3.8, corrugations in the initial conditions due to the fact that the interface is not aligned 

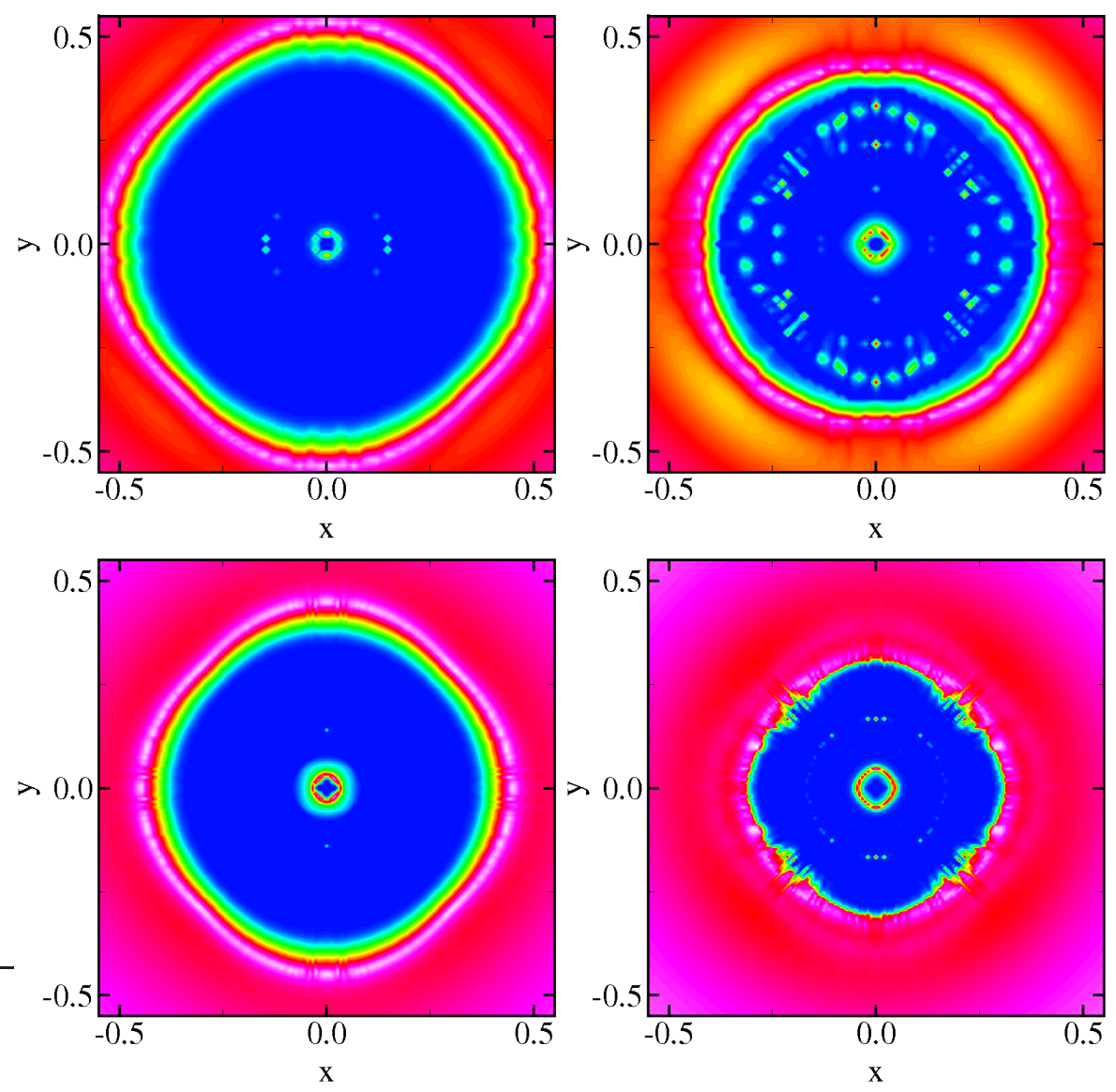

Figure 4.14: Numerical Schlieren contours for Rayleigh collapse for $\Delta x=1.33 \times$ $10^{-2}$ (top) and $\Delta x=6.67 \times 10^{-3}$ (bottom), at $t c_{L} / R_{o}=7.46$ (left) and $t c_{L} / R_{o}=$ 7.63 (right).

with the grid develop into interface perturbations. This phenomenon on a larger scale is further considered as a mechanism of jet formation in Section 4.4.

A possible mechanism for the Richtmyer-Meshkov instability is the interaction between the internal shock (generated from the initial conditions) with the collapsing interface. However, the impedance of the liquid is so large that it acts almost like a solid wall and completely reflects the shock back into the bubble. On a larger scale, the interaction of the incoming shockwave with the bubble leads to Richtmyer-Meshkov instability, as described in Section 4.4. However, RichtmyerMeshkov instability plays a role on a large scale in the formation of the re-entrant 
jet, as described in the next sections.

Contrary to the corresponding problem in gases shown in Section 3.6.4, the Kelvin-Helmholtz instability is not particularly pronounced in shock-induced bubble collapse because the collapse time is too short for such vortical structures to develop.

\subsubsection{Measure of non-sphericity}

A quantitative measure of the bubble non-sphericity is given by the ratio of the projected bubble area to the length of the perimeter, as described by equation C.8 in Appendix C. A value of 0.5 corresponds to a spherical collapse, while a value of zero implies that the interface has greatly deformed. Figure 4.15 shows the history of the non-sphericity for Rayleigh collapse in a free-field and near a wall $\left(H_{o} / R_{o}=1.5, \infty, p_{s} / p_{o}=353,714\right)$ and shock-induced collapse in a free-field and near a wall $\left(H_{o} / R_{o}=1.5, \infty, p_{s} / p_{o}=353\right)$. The results are plotted until collapse.

In all cases, the initial portion of the collapse is spherical. The shape becomes non-spherical only late in the collapse and reaches a most non-spherical shape at collapse. Instabilities develop even during the free-field Rayleigh collapse, so that the bubble does not remain completely spherical and is most non-spherical at collapse. The maximum non-sphericity depends greatly on the initial pressure ratio for shock-induced collapse and on the initial stand-off distance, for Rayleigh collapse near a wall, as shown Figure 4.16.

The bubble non-sphericity defined in Equation C.8 is a difficult quantity to compute numerically, as illustrated by the scatter in the results for free field shockinduced collapse. Nevertheless, the overall behavior is expected, as a collapse driven by a larger pressure ratio or closer to the wall leads to a more non-spherical shape. This quantity is even more difficult to measure experimentally. Another measure of non-sphericity of the collapse that is less difficult to measure in experiments is the sum of the velocities of the jet and distal side, $v_{j}+v_{d}$, as described in Section 4.3.5. 


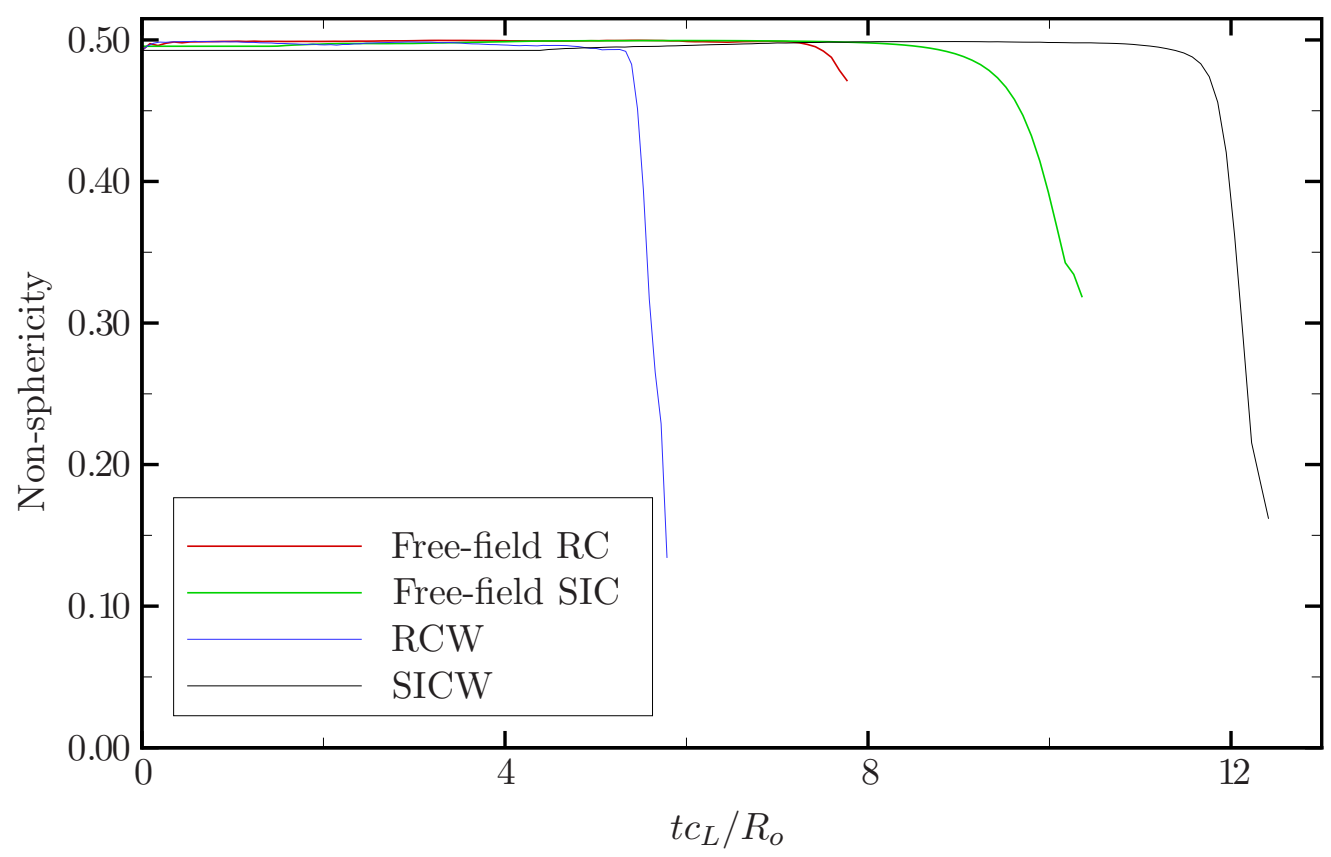

Figure 4.15: History of the bubble non-sphericity for Rayleigh collapse $\left(p_{s} / p_{o}=\right.$ $353,714)$ and shock-induced collapse $\left(p_{s} / p_{o}=353\right)$ in a free-field and near a wall $\left(H_{o} / R_{O}=1.5\right)$.

\subsubsection{Maximum jet velocity}

The maximum jet velocity, $v_{j}$, is an important quantity in non-spherical bubble collapse. As described in Section 4.5.2, when the jet hits the distal side, which moves at some velocity, $v_{d}$, a water-hammer pressure is generated, with significant potential for damage (Huang et al., 1973). In the present problems, the jet velocity is independent of the initial bubble radius because there is no additional length scale associated with the problem. As shown in Section 5.1, this is not true in shockwave lithotripsy, where the pulse has a finite width.

\section{Free-field collapse}

Figure 4.17 shows the maximum velocity of the jet and distal side as a function of the initial pressure ratio for free-field Rayleigh collapse and shock-induced collapse. The difference and sum of the velocities are also plotted. For Rayleigh collapse, 

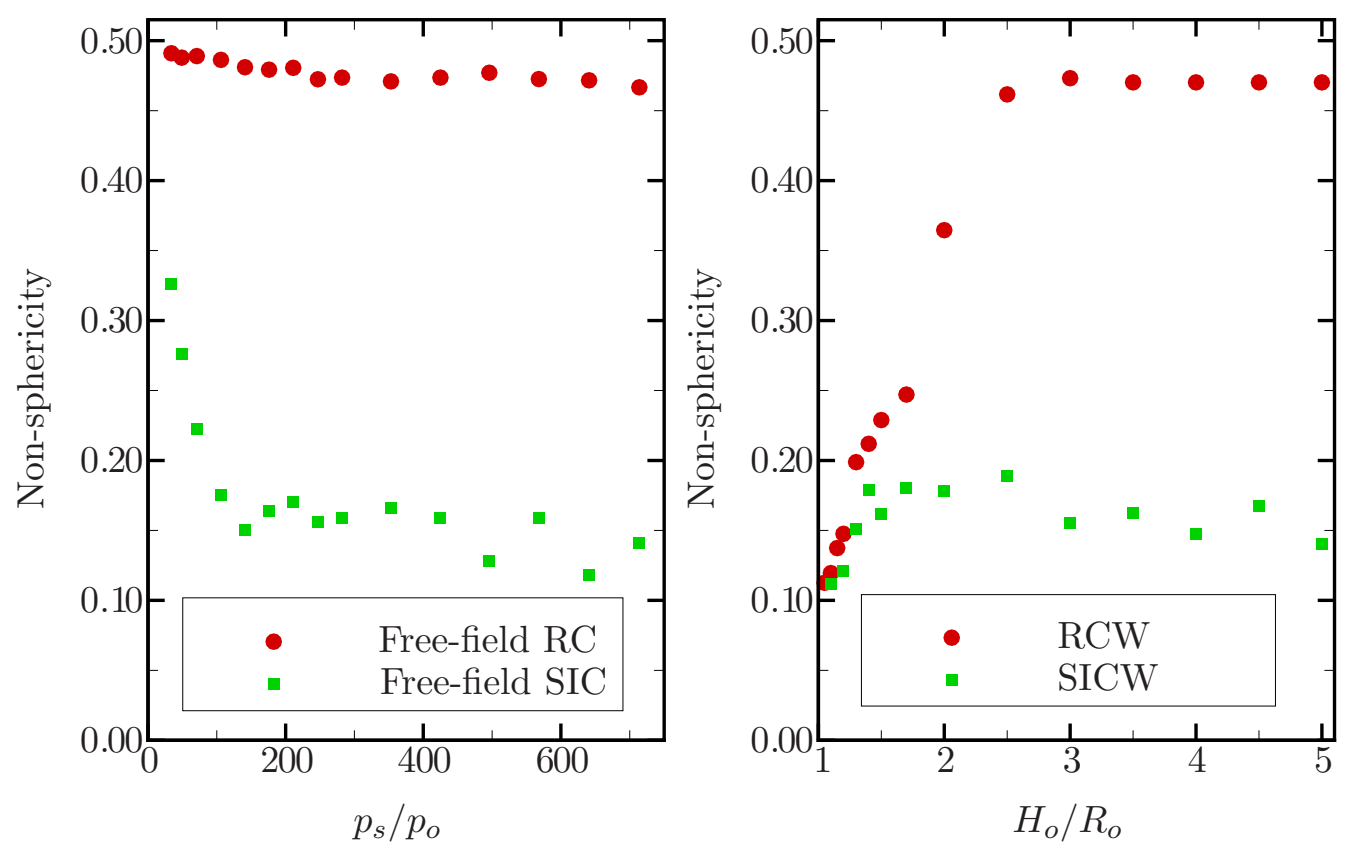

Figure 4.16: Maximum bubble non-sphericity as a function of the pressure ratio for free-field Rayleigh collapse and shock-induced collapse (left), and of the initial stand-off distance for shock-induced collapse with $p_{s} / p_{o}=353$ and Rayleigh collapse near a wall with $p_{s} / p_{o}=714$ (right).

there is no jetting; the velocity is that of the bubble wall.

As expected, the velocities of the jet and distal side increase with the initial pressure ratio. In Rayleigh collapse, $v_{j}=-v_{d}$, by symmetry; in shock-induced collapse, $\left|v_{j}\right| \gg\left|v_{d}\right|$ for reasons explained in Section 4.4.4. High jet velocities are achieved (up to $1250 \mathrm{~m} / \mathrm{s}$ for the highest pressure ratio); for pressure ratios representative of SWL, $v_{j} \approx 1000 \mathrm{~m} / \mathrm{s}$. The velocity difference is used in the calculation of the water-hammer pressure (Section 4.5.2). Although not shown here, the maximum jet velocity is observed slightly before the minimum volume is reached because of the finite time it takes for it to slow down as it is about to impact the distal side. 


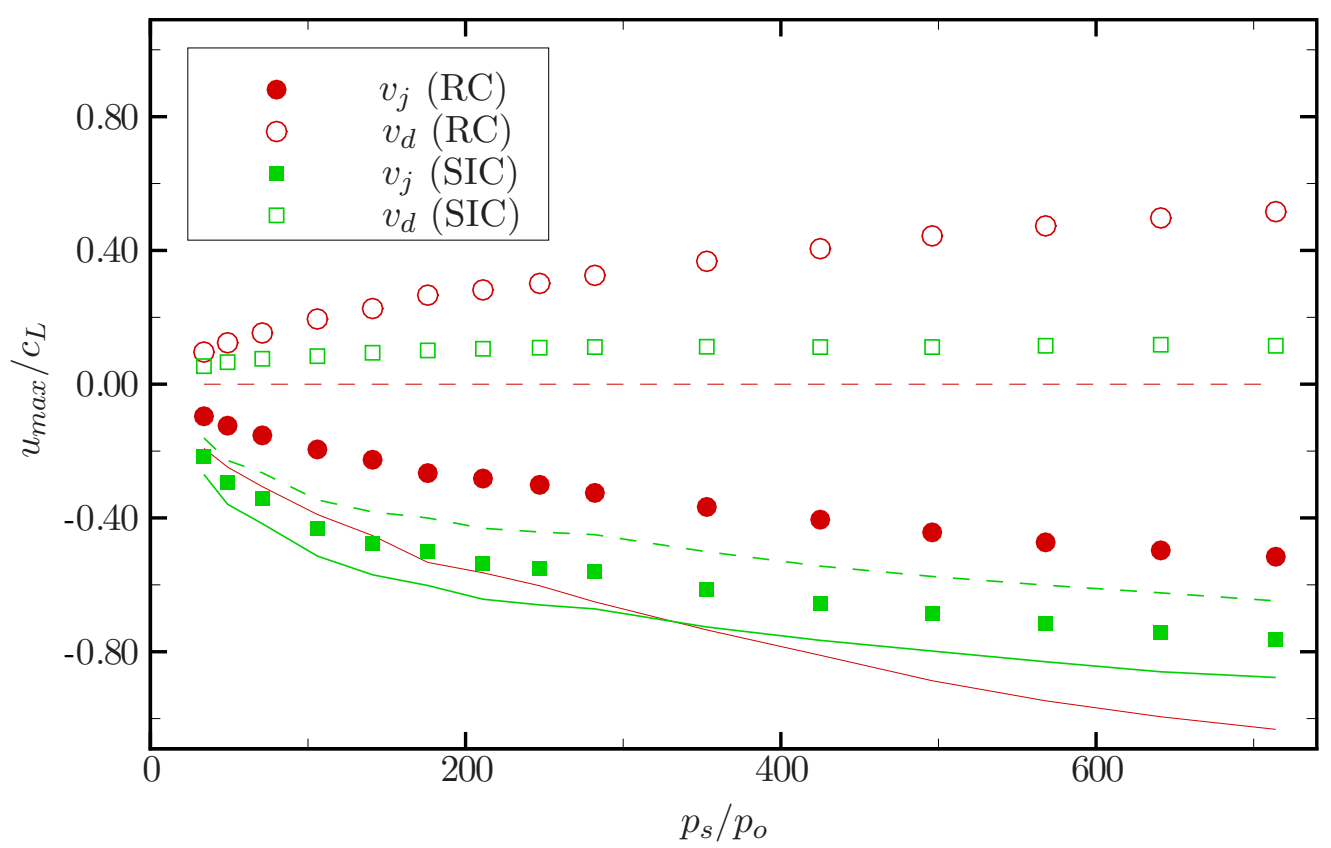

Figure 4.17: Maximum velocity of the jet and distal side for free-field Rayleigh collapse and shock-induced collapse. The dashed line shows the sum of the velocities and the solid line the relative velocities.

\section{Collapse near a wall}

Figure 4.18 shows the maximum velocity of the jet and distal side, as a function of the initial stand-off distance, for Rayleigh collapse with a pressure ratio of $p_{s} / p_{o}=34$. Results from the experiments of Philipp \& Lauterborn (1998) and the simulations of Plesset \& Chapman (1971), Blake et al. (1986), and Zhang et al. (1993) are included.

The present simulations show that the jet velocity increases as the initial standoff distance to the wall decreases. As the stand-off distance is increased, the behavior becomes closer to that of free-field Rayleigh collapse, thus suggesting that a more non-spherical collapse leads to a higher jet velocity. The maximum jet velocity measured in the computations is $v_{j}=107 \mathrm{~m} / \mathrm{s}$, at $H_{o} / R_{o}=1.05$. The experiments and some of the prior simulations show different trends. In the experiments, the maximum jet velocity is measured by calculating the slope of 


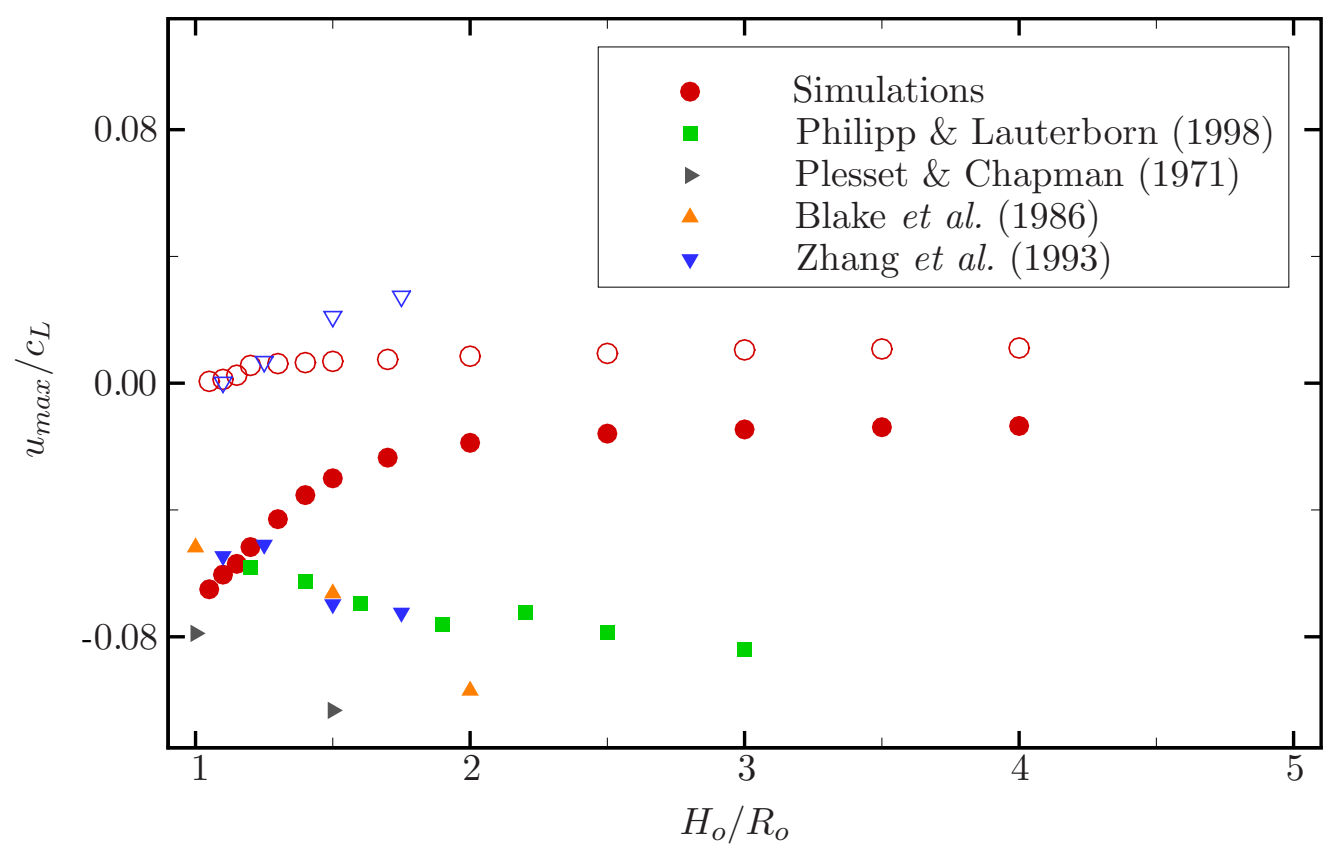

Figure 4.18: Maximum velocity of the jet and distal side as a function of the initial stand-off distance for Rayleigh collapse near a wall $\left(p_{s} / p_{o}=34\right)$. Empty symbols: the velocity of the distal side of the corresponding filled symbols.

the position of the jet; the error bars in the original article are large, and such measurements are difficult to make precisely from photographs. There is scatter in the prior numerical simulations results as well. The main reason for the lower jet velocity (and possibly the different trend) in the present simulations of Rayleigh collapse is that the bubble initially contains a large amount of non-condensible gas, as described in Section 2.2.5. Thus, the bubble does not achieve as small a volume because the collapse is arrested by the gas compression sooner. In this case, the conditions at collapse for this case may not be exactly the same as those in nature. These issues are discussed in greater detail in Section 4.5.4.

Figure 4.19 shows the velocity of the jet and distal side, as a function of the initial stand-off distance, for shock-induced collapse $\left(p_{s} / p_{o}=353\right)$ and Rayleigh collapse $\left(p_{s} / p_{o}=714\right)$ near a wall. The velocities (jet and distal side) asymptote to the values of the free-field Rayleigh collapse and shock-induced collapse with 


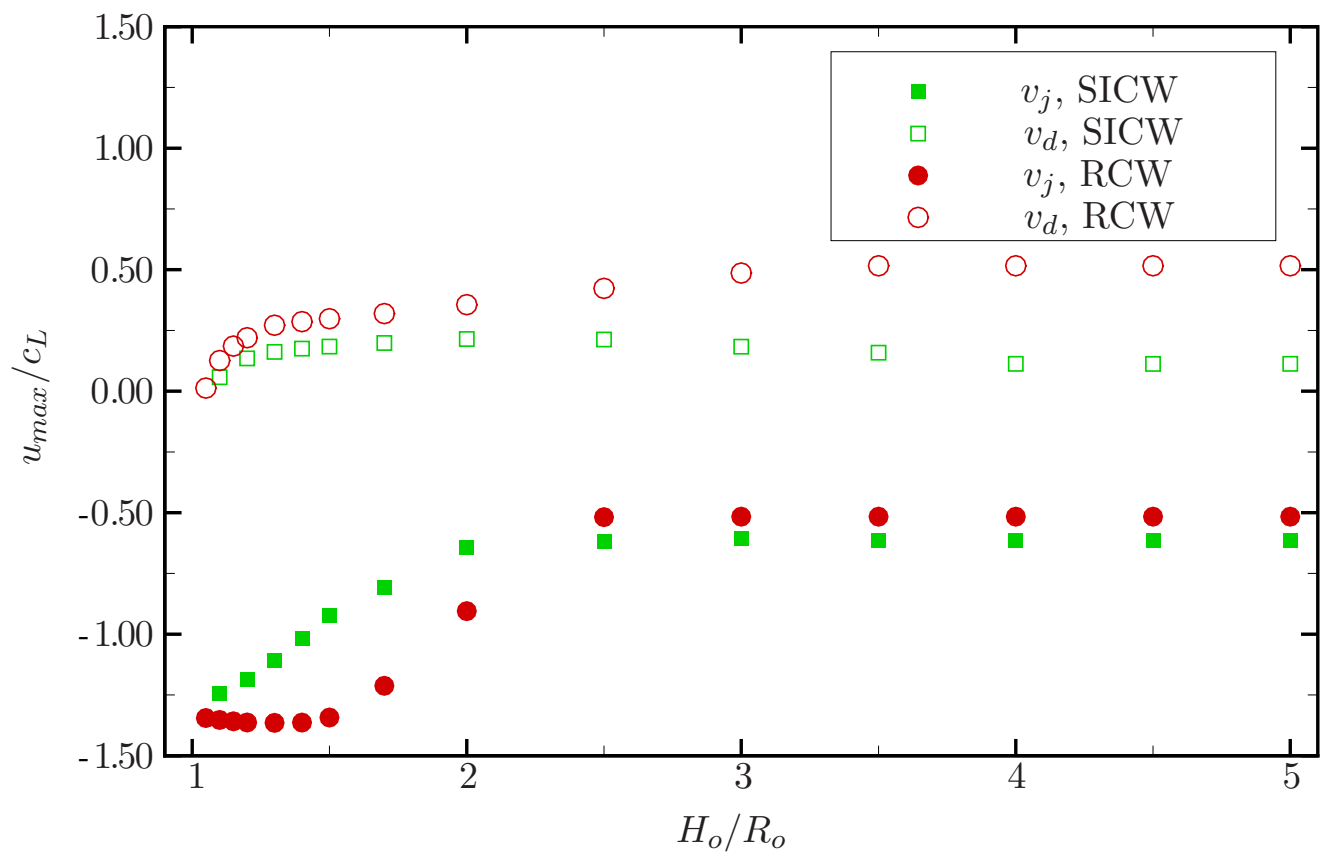

Figure 4.19: Maximum velocity of the jet and distal side as a function of the initial stand-off distance for shock-induced collapse near a wall $\left(p_{s} / p_{o}=353\right)$.

the same pressure ratio (see Figure 4.17). Near the wall, the jet velocity increases dramatically and reaches a maximum of $2250 \mathrm{~m} / \mathrm{s}$ for RCW and $2047 \mathrm{~m} / \mathrm{s}$ for SICW. These results are consistent with those obtained by Jamaluddin (2005). Interestingly, the maximum (near the wall) and asymptotic (far from the wall) jet velocities are similar in both cases, though the distribution within $H_{o} / R_{o} \approx 1.1-$ 2.0 shows significant discrepancies. The velocities of the distal side are similar near the wall, as expected since the pressure doubling occurs almost instantaneously there, but then diverge larger $H_{o} / R_{o}$, since the shock does not have time to reflect back to the bubble and thus affect the collapse. A similar behavior is observed in the collapse time (Section 4.3.7).

\subsubsection{Jet size}

An important reason for computing the characteristic size of the jet is to determine the flow regime that occurs within the jet. Because high curvatures and small 
scales are achieved, viscous and surface tension effects may be important. In order to determine the characteristic size of the jet, free-field shock-induced collapse is considered. Figure 4.20 shows the outline of the bubble for two different shock pressure ratios, $p_{s} / p_{o}=71,282$.
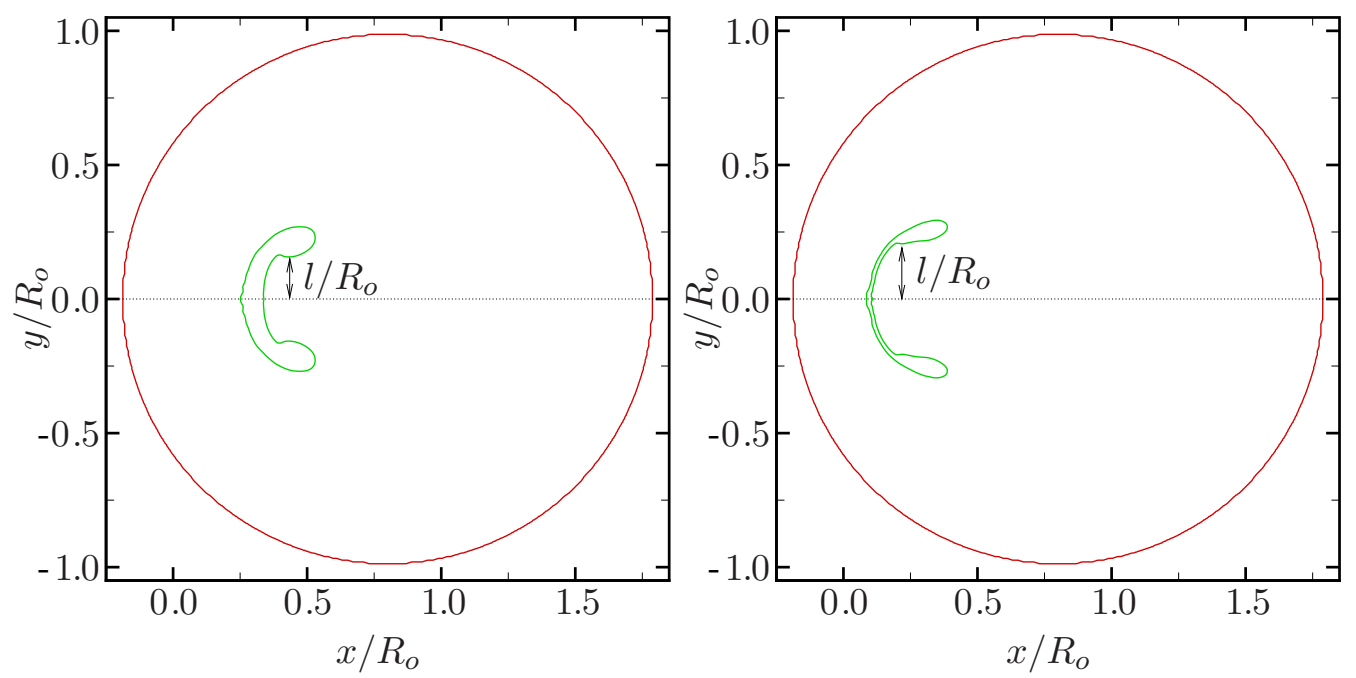

Figure 4.20: Jet outline for free-field shock-induced collapse for $p_{s} / p_{o}=71$ at $t c_{L} / R_{o}=20.1$ (left) and for $p_{s} / p_{o}=282$ at $t c_{L} / R_{o}=11.0$ (right).

The characteristic size of the jet $l$ is taken as the length between the $x$-axis and the point where the jet outline first becomes horizontal, as shown by the arrows in Figure 4.20. In both cases, this occurs just before the bubble achieves its minimum volume. The shape of the bubble at that time depends on the pressure ratio across the shock: for a higher pressure ratio, the jet becomes wider, more similar to the experiments of Bourne \& Field (1992), in contrast to the results for lower pressure ratios, which lead to a more prominent vortex ring structure similar to the shockbubble interaction in gases of Haas \& Sturtevant (1987).

As the pressure ratio across the shock increases, both the width of the jet and the size of the bubble at that time increase, as seen in Figure 4.21, which shows the jet size and the height of the bubble (top of the bubble in Figure 4.20) as a function of the pressure ratio across the shock. The aspect ratio of the bubble becomes more elongated in the vertical direction in Figure 4.20 as the shock Mach 
number is increased.

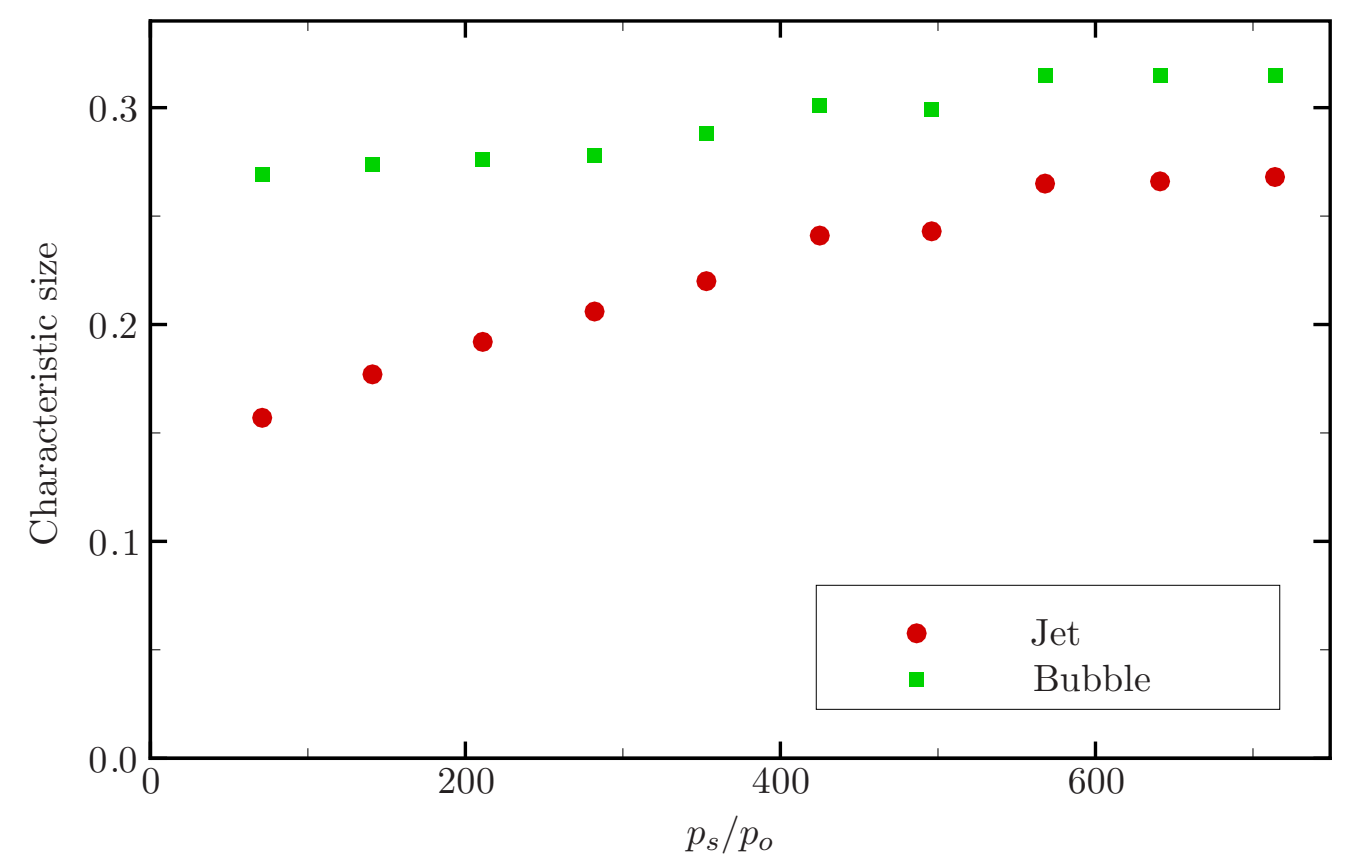

Figure 4.21: Jet size and bubble height as a function of the pressure ratio for free-field shock-induced collapse.

Based on the maximum jet velocities attained in Figure 4.17 and on the jet size shown in Figure 4.21, Reynolds numbers ranging from $8.80 \times 10^{2}-3.37 \times 10^{3}$ and Weber numbers ranging from $6.79 \times 10^{3}-5.79 \times 10^{4}$ are achieved for a bubble with a $10 \mu \mathrm{m}$ radius. However, at the instant shown in Figure 4.20, the jet has already decelerated by approximately $30 \%$ from its maximum value, so that the effective Reynolds number may range from $2.50 \times 10^{2}-1.04 \times 10^{3}$ and the effective Weber number may range from $2.04 \times 10^{3}-1.74 \times 10^{4}$. Thus, viscous and surface tension effects may be important locally for small bubbles.

\subsubsection{Collapse time}

The collapse time of a bubble strongly depends on the pressure ratio driving the collapse, as suggested by Equation 4.8. Figure 4.22 shows a comparison between the collapse time computed from the Rayleigh equation 4.4 and the results of the 
numerical simulations for free-field Rayleigh collapse and shock-induced collape. As discussed earlier, the bubble volume is not always spherical in the simulations, so that the minimum volume is considered to determine the collapse time. The time origin in shock-induced collapse is taken as the time when the shock first hits the bubble.

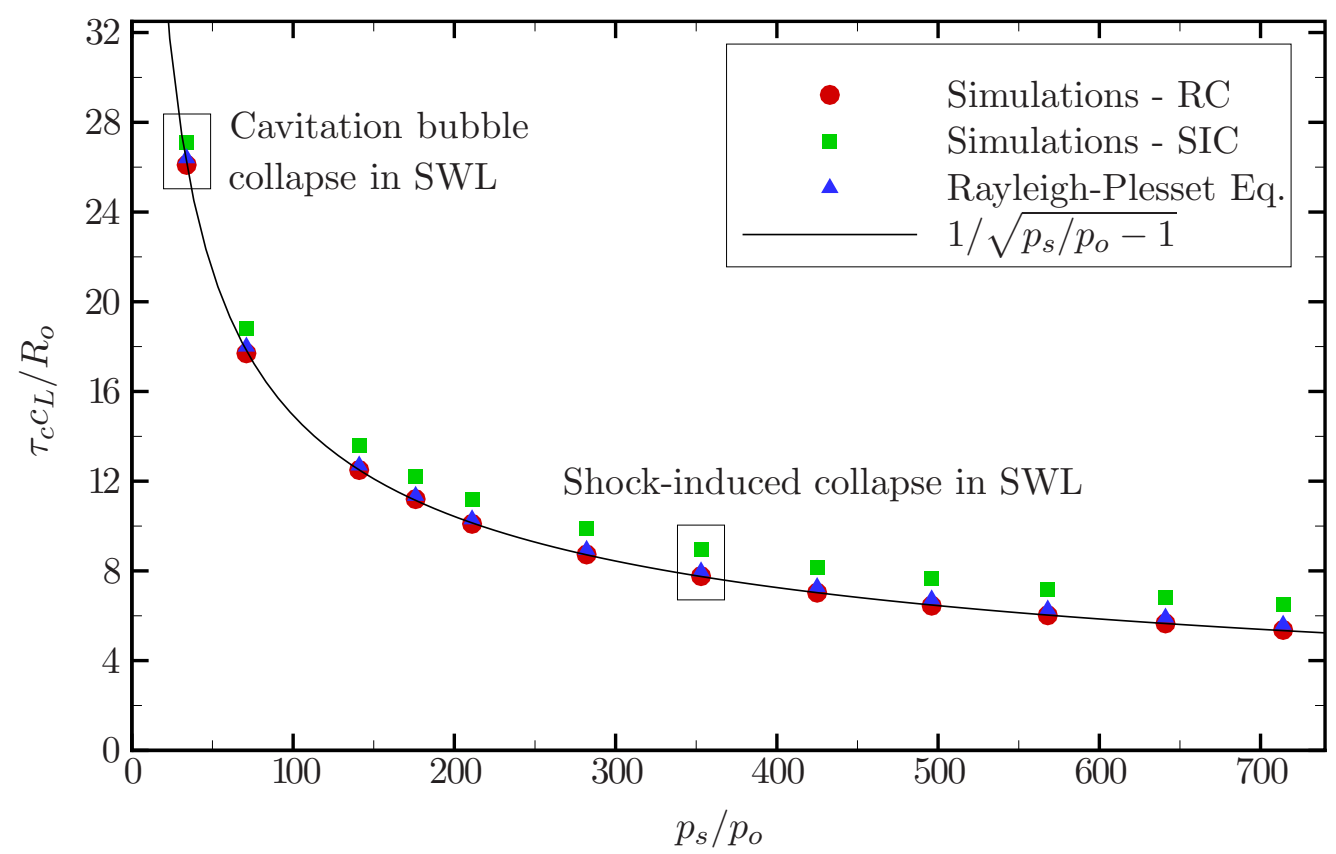

Figure 4.22: Collapse time as a function of the initial pressure ratio for free-field Rayleigh collapse and shock-induced collapse.

The agreement between the present results and the solution to Equation 4.4 is very good. Though not manifest here, compressibility effects lead to a slight deviation from the Rayleigh-Plesset curve for higher pressure ratios. The collapse time for shock-induced collapse is approximately one time unit greater than that of Rayleigh collapse for the same pressure ratio. This effect accounts for the time it takes for the shockwave to propagate over the span of the bubble; if the collapse time is taken as the average between the collapse time when the shock first hits the bubble and that when the shock ends its diffraction about the bubble, the collapse times for Rayleigh collapse and shock-induced collapse are approximately 
equal. The collapse time scales with $1 / \sqrt{p_{s} / p_{o}-1}$, as expected from the Rayleigh equation 4.4 .

The presence of the wall retards the flow of liquid filling the void created by the collapsing bubble, so that the overall collapse is slower. Based on a perturbation analysis of the Rayleigh-Plesset equation, Rattray (1951) derived the following first-order correction for this effect:

$$
\tau_{r}=\tau_{c}\left(1+0.205 \frac{R_{o}}{H_{o}}\right)+O\left(\left(\frac{H_{o}}{R_{o}}\right)^{2}\right)
$$

From a series of high-speed photographs, Vogel et al. (1988) calculated a prolongation factor, $k_{1}$, that depends on the initial stand-off distance and ranges between $1-1.3$. Thus, for bubble collapse near a wall, $t_{12}=2 \tau_{c} k_{1}$, where $t_{12}$ is the time between the generation of the bubble and the collapse, which is determined by measuring the time between the acoustic signal of each event.

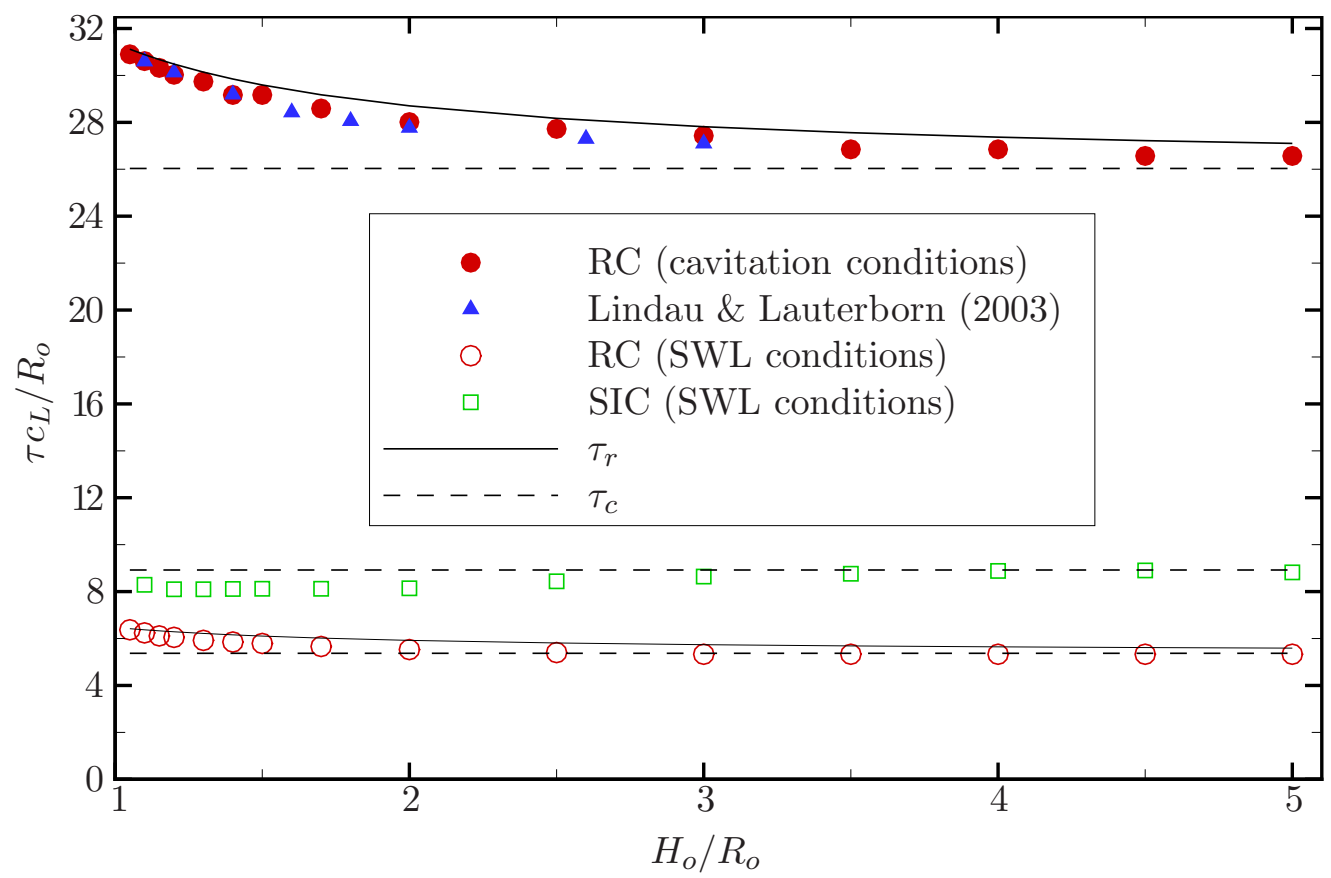

Figure 4.23: Collapse time as a function of the initial stand-off distance for Rayleigh collapse and shock-induced collapse near a wall $\left(p_{s} / p_{o}=34\right.$ for cavitation conditions and $p_{s} / p_{o}=353$ for SWL conditions). 
Figure 4.23 shows the collapse time as a function of the initial stand-off distance computed from the results of Rattray (1951), Vogel et al. (1988) and from the present computations. The experimental collapse time is computed by using the tabulated data in Lindau \& Lauterborn (2003). ${ }^{6}$ The top set of data corresponds to cavitation bubble collapse $\left(p_{s} / p_{o}=34\right)$, while the bottom set relates the shockinduced collapse $\left(p_{s} / p_{o}=353\right.$ for shock-induced collapse, $p_{s} / p_{o}=714$ for Rayleigh collapse).

As expected, the presence of the wall delays the collapse. The presence of the wall is communicated to the bubble by the reflection of the expansion wave released by the initial Riemann problem. Consequently, the pressure driving the collapse is reduced as the expansion propagates over the bubble, thus leading to a longer collapse time. This phenomenon also occurs in incompressible simulations, in which the effect is felt instantaneously due to the effective infinite speed of propagation of the wave. The agreement between the present results and the experiments is very good in Rayleigh collapse under cavitation conditions. However, the model of Rattray (1951) slightly overpredicts the results. In shock-induced collapse, shock reflection off the wall results in pressure doubling, so that the effective pressure ratio driving the collapse is the same as for Rayleigh collapse with $p_{s} / p_{o}=714$. Close to the wall, the pressure doubles almost instantaneously, so that the delay between free-field shock-induced collapse and Rayleigh collapse observed in Figure 4.23 agrees with the results shown in Figure 4.22. For bubbles farther from the wall, the collapse starts when the shock first impacts the bubble. Thereafter, the shock must travel twice the stand-off distance before interacting with the bubble again, at which point the bubble has already started its collapse due to the initial compression caused by the incoming shock. The doubling then only affects a small portion of the collapse. Thus, as the initial stand-off distance increases, the collapse time tends to that of free-field shock-induced collapse with $p_{s} / p_{o}=353$, for which $\tau_{c}=8.92$. Since the shock Mach number is close to unity, the collapse of

\footnotetext{
${ }^{6}$ Because the initial bubble pressure is initially assigned atmospheric value in the simulations (see Section 2.1.1), a scaling factor of $\sqrt{p_{a t m} / p_{v}}$ is used to compare to experiments.
} 
bubbles farther away than $H_{o} / R_{o} \approx 4.5$ are unaffected by pressure doubling. This analysis shows that the collapse time in shock-induced collapse is of the same order as the time it takes for the shock to propagate within the liquid. The expected behavior is achieved for Rayleigh collapse with $p_{s} / p_{o}=714$.

\subsubsection{Displacement of the bubble centroid}

Theory and experiments have shown that the bubble moves towards the wall as it collapses (Benjamin \& Ellis, 1966; Plesset \& Chapman, 1971). In order to preserve its Kelvin impulse during collapse (Vogel et al., 1989), the bubble accelerates towards the wall. In shock-induced collapse, the bubble migrates in the direction of propagation of the shockwave because of the fluid entrainement behind the shock. The history of the bubble displacement in Rayleigh collapse is compared to the experiments of Vogel et al. (1989) in Figure 4.24. In the experiments, $H_{o} / R_{o}=2.15$, while $H_{o} / R_{o}=2.0$ in the simulation.

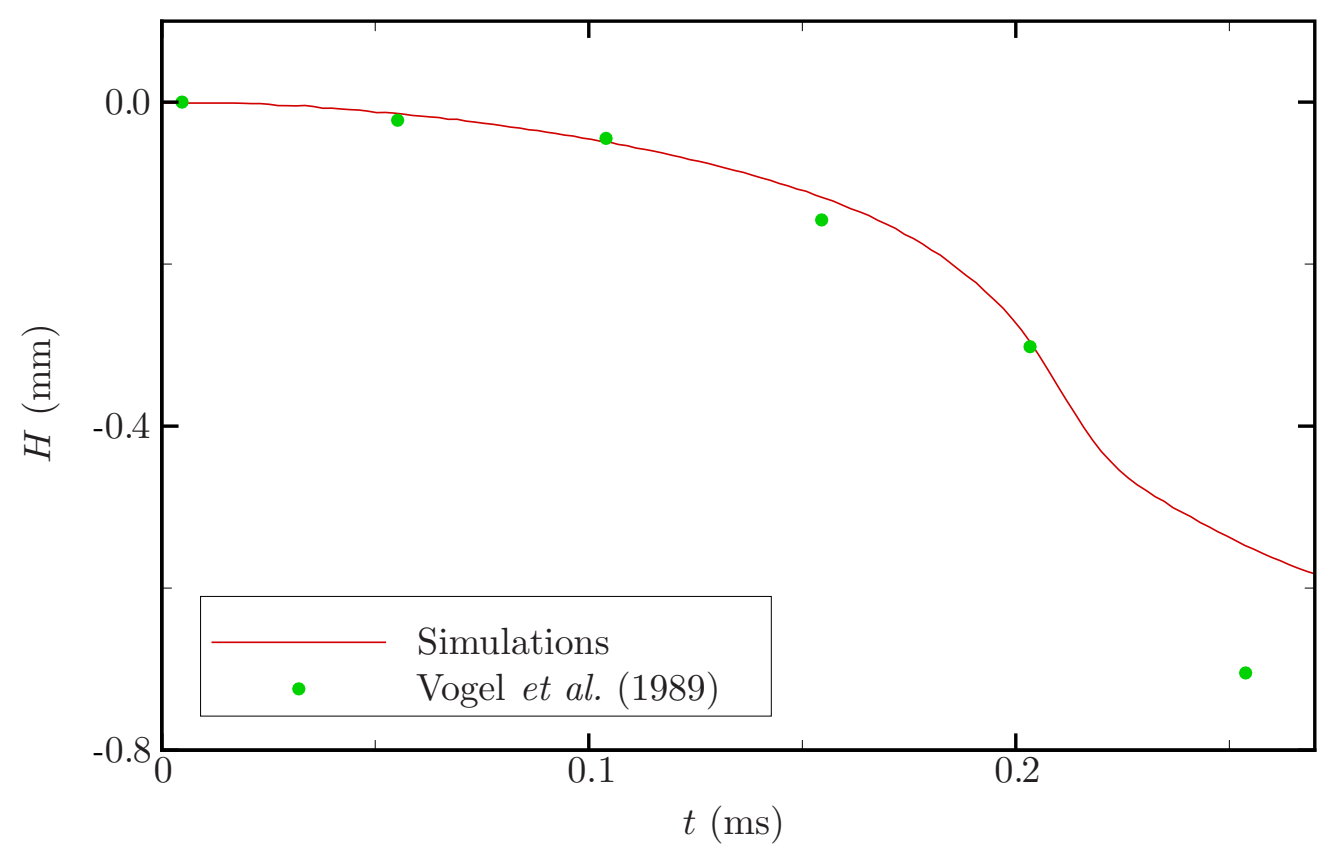

Figure 4.24: History of the bubble displacement for Rayleigh collapse near a wall (simulations: $H_{o} / R_{o}=2.0$; experiments by Vogel et al. $(1989): H_{o} / R_{o}=2.15$ ). 
The initial behavior of the bubble is well captured until collapse $\left(\tau_{c} \approx 0.2 \mathrm{~ms}\right)$. Early in the collapse, the bubble moves slowly towards the wall. Its motion then progressively accelerates during the collapse. At collapse, the bubble has migrated by a significant amount towards the wall. The impact of the jet on the distal side further accelerates the bubble and causes it to take the form of a vortex ring, which convects towards the wall. The numerical results diverge from the experimental results thereafter, because certain effects important at collapse are not modeled (e.g., mass transfer). As noted in the experiments of Tomita \& Shima (1986) and Vogel et al. (1989), the bubble moves closer to the wall with each collapse.
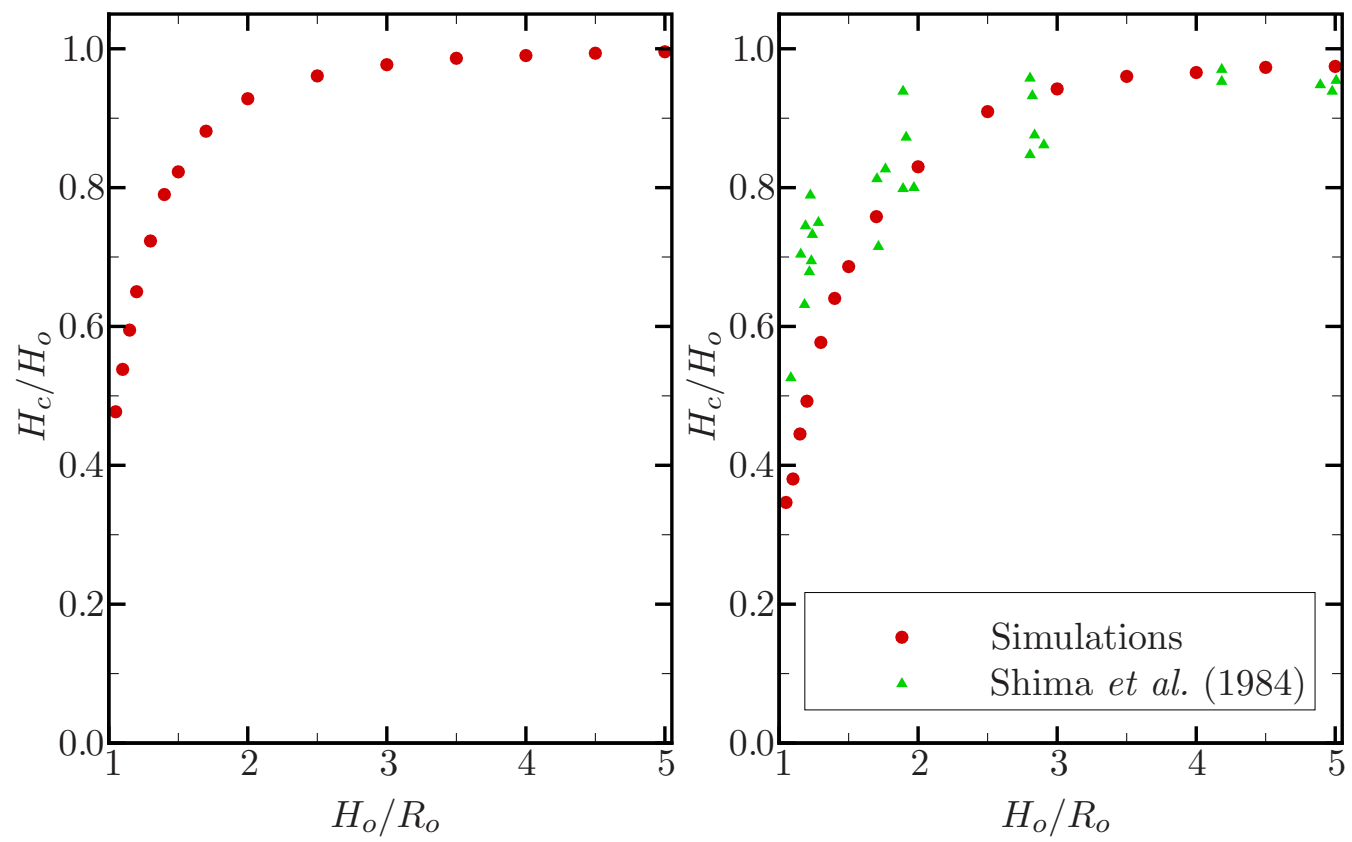

Figure 4.25: Distance of the bubble from the wall at collapse for Rayleigh collapse with $p_{s} / p_{o}=34$ (left) and shock-induced collapse with $p_{s} / p_{o}=25$ (right) as a function of the initial stand-off distance.

The position of the bubble centroid at collapse is the origin of the emitted shockwave. Since the amplitude of the shock generated upon collapse is expected to decrease with distance (see Section 4.5.4), the consequence of this migration is that the measured wall pressure is higher (Tomita \& Shima, 1986), even though 
a collapse near the wall is gentler. Figure 4.25 shows the bubble displacement at collapse for Rayleigh collapse (under cavitation conditions) and for shock-induced collapse with $p_{s} / p_{o}=25$ in order to compare to the experiments of Shima et al. $(1984) .^{7}$

In Rayleigh collapse, the influence of the wall is small on bubbles that are initially far away, such that their centroid does not migrate significantly. Bubbles initially close to the wall show a larger displacement towards the wall. In shockinduced collapse, there is a net velocity towards the wall after the passage of the shock. However, there is no effective flow after the shock reflection off the wall. Thus, the displacement is larger in the case of shock-induced collapse, especially for larger $H_{o} / R_{o}$. As in Rayleigh collapse, a bubble initially close to the wall feels its effect more significantly and migrate towards it by a larger amount. The simulations agree well with the experiments. Further comparisons are made with the experiments of Shima et al. (1984) in Section 4.5.4.

\subsubsection{Bubble circulation}

In the non-spherical bubble collapse considered in the present work, baroclinic vorticity is generated due to the misalignment of the pressure and density gradients present during the interaction between various types of waves and the bubble interface. The vorticity equation for inviscid flow is written as

$$
\frac{\partial \boldsymbol{\omega}}{\partial t}+(\mathbf{u} \cdot \nabla \boldsymbol{\omega})=(\boldsymbol{\omega} \cdot \nabla) \mathbf{u}-\boldsymbol{\omega}(\nabla \cdot \mathbf{u})+\frac{\nabla \rho \times \nabla p}{\rho^{2}}
$$

This expression can be integrated over the whole domain to obtain an equation for the time rate of change of circulation for shock-bubble interactions (Haas \& Sturtevant, 1987):

$$
\Gamma_{t}=\int_{S} \frac{\nabla \rho \times \nabla p}{\rho^{2}} \cdot d \mathbf{S}
$$

\footnotetext{
${ }^{7}$ The shock amplitude in the experiments of Shima et al. (1984) is $5 \mathrm{MPa}$, which is the value measured upon reflection off the wall. Thus, the amplitude of the incoming shock in the present simulations is $2.5 \mathrm{MPa}$.
} 
where the area is a slice through the domain in the radial direction, through $r=0$. The circulation is computed over the top-half domain, since, by symmetry, the bottom half has the opposite sign. From Equations 4.30 and 4.31, all the circulation generated in the present problems is expected to be caused by baroclinic vorticity. In theory, because the gradients of pressure and density are infinite, the resulting vorticity field consists of a vortex sheet along the interface. However, because of the numerical diffusion in the simulations, the vorticity is spread over a wider area along the interface and the gradients are spread over a few grid points. Figures 4.26 and 4.27 show the total circulation and rate of change of circulation for free-field shock-induced collapse $\left(p_{s} / p_{o}=353\right)$ and Rayleigh collapse near a wall $\left(p_{s} / p_{o}=353, H_{o} / R_{o}=1.5\right)$. In addition, the positive and negative contributions to the circulation are plotted.
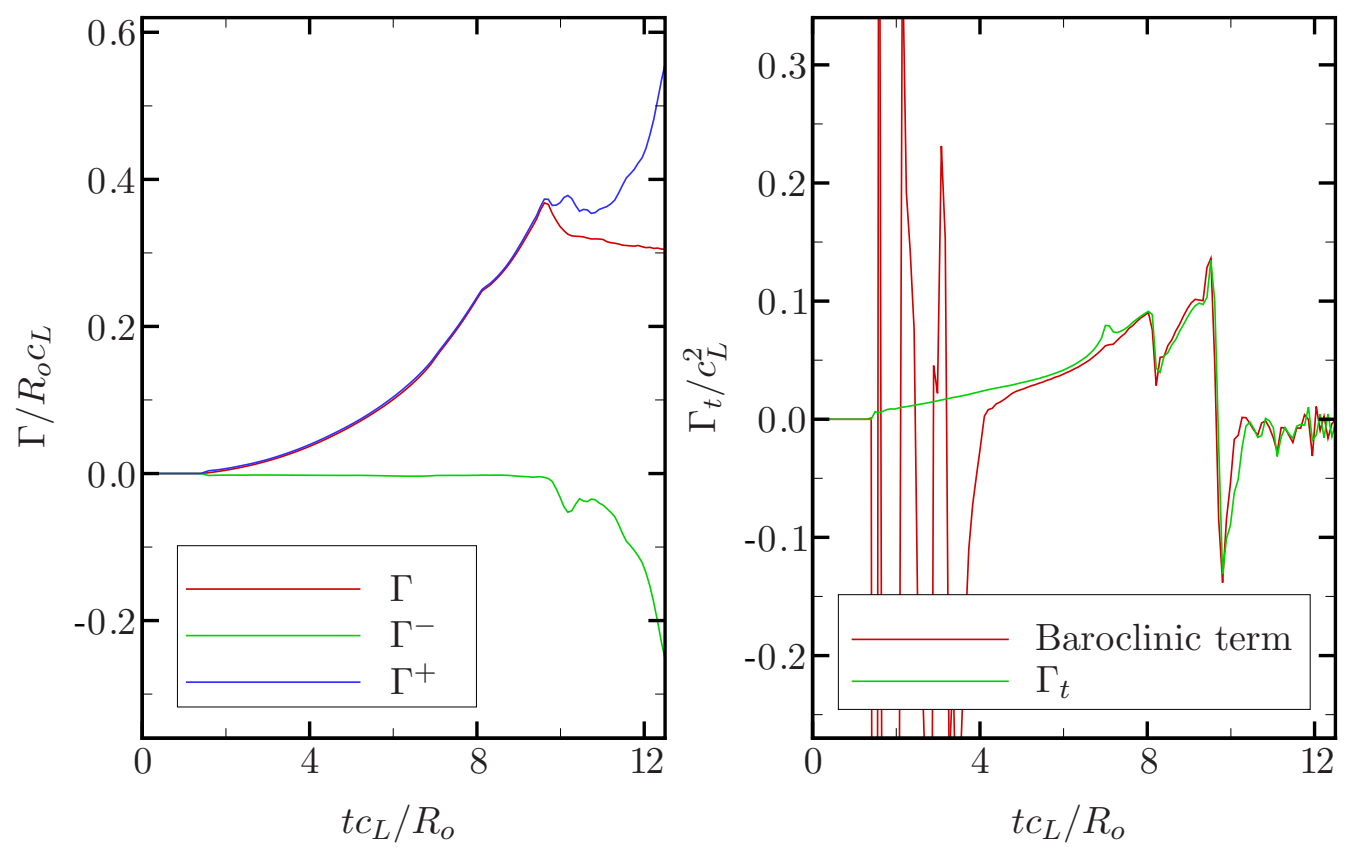

Figure 4.26: Total circulation (left) and rate of change of the circulation (right) for free-field shock-induced collapse $\left(p_{s} / p_{o}=353\right)$.

In both problems, all of the circulation is due to baroclinic vorticity, as the transmitted wave propagates within the bubble. Furthermore, primarily negative 
(clockwise) vorticity is generated until collapse. The circulation increases until it reaches a maximum before the rebound. In both cases, the continuous increase in circulation may be due to vortex-accelerated vorticity deposition (Peng et al., 2003), where baroclinic vorticity is generated due to the acceleration of the bubble interface. When the bubble volume decreases, the amplitude of the internal shockwaves increases because of focusing and the intensity of the vortex sheet increases. The interactions becomes very complex in the last stages of collapse.
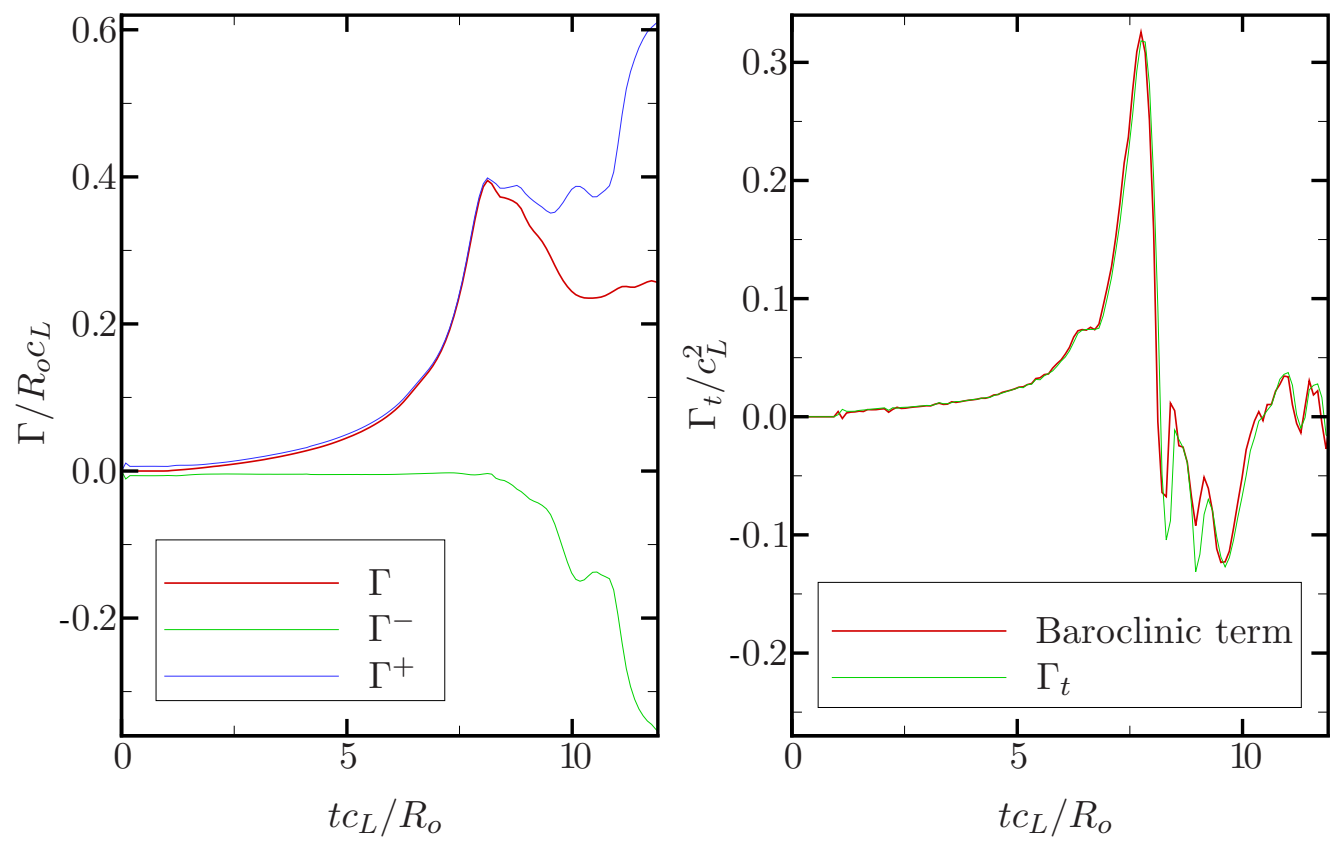

Figure 4.27: Total circulation (left) and rate of change of the circulation (right) for Rayleigh collapse near a wall, $\left(p_{s} / p_{o}=714, H_{o} / R_{o}=1.5\right)$.

In shock-induced collapse, the baroclinic vorticity is generated due to the interaction between the shockwave and the interface. The transmitted shock propagates through the bubble, generating negative vorticity. The small deviation in $\Gamma_{t}$ at $t c_{L} / R_{o} \approx 7$ is due to the (non-spherical) convergence of the shock within the bubble. After the shock reflects off the distal side at $t c_{L} / R_{o} \approx 8$, positive vorticity is generated as the internal shock propagates in the opposite direction, thereby decreasing the circulation. However, the circulation continues to increase after 
this process, possibly due to vortex-accelerated vorticity deposition (Peng et al., 2003). The large initial oscillations in the rate of change of circulation are caused by the sharp pressure gradient across the shock.

In Rayleigh collapse near a wall, baroclinic vorticity is generated due to the interaction between the initial expansion that reflects off the wall and the interface. The transmitted expansion wave propagates through the bubble, thus generating negative vorticity. The kink observed at time $t c_{L} / R_{O} \approx 6.5$ is caused by the reflection of the internal shock off the distal side. As the wave propagates back, vorticity of the opposite sign is generated, thus causing the decrease. This process then continues upon each reflection. The circulation increases rapidly as the bubble enters the fast collapse regime.

Based on Equation 4.31, it is expected that in shock-induced collapse the circulation increases linearly with $p_{s} / p_{o}$ and decreases linearly with $\rho_{B} / \rho_{L}$. Figure 4.28 shows the circulation after the propagation of the internal shock through the bubble as a function of the pressure ratio (holding the density ratio constant at $\rho_{B} / \rho_{L}=1.22 / 998$ ) and density ratio (holding the pressure ratio constant at $\left.p_{s} / p_{o}=353,641\right)$. In the pressure plot, the maximum circulation and the circulation when the internal shock hits the distal side are included.

As expected from Equation 4.31, the circulation after the passage of the shock increases linearly with the pressure ratio. The maximum circulation does not follow this trend initially, but later does. As discussed in Section 4.3.2, the speed of the transmitted shock does not change significantly with the pressure compared to the jet speed. For a small pressure ratio, the jet speed is low enough that the transmitted shock can reflect internally several times. For larger pressure ratios, the jet speed becomes so large that it almost catches up with the shock, so that the maximum circulation occurs just around when the shock hits the distal side. These observations can be related to the density plot. A departure from the linear relationship in $\Gamma_{\max }$ is observed in cases where the transmitted shock has had time to reflect multiple times within the bubble before the jet hits the distal side (low pressure ratio, high density contrast). In the plot of the density, the linear 
dependence appears to hold if the density contrast is not too large. If the density is smaller than that of air, there will be little difference in the circulation.
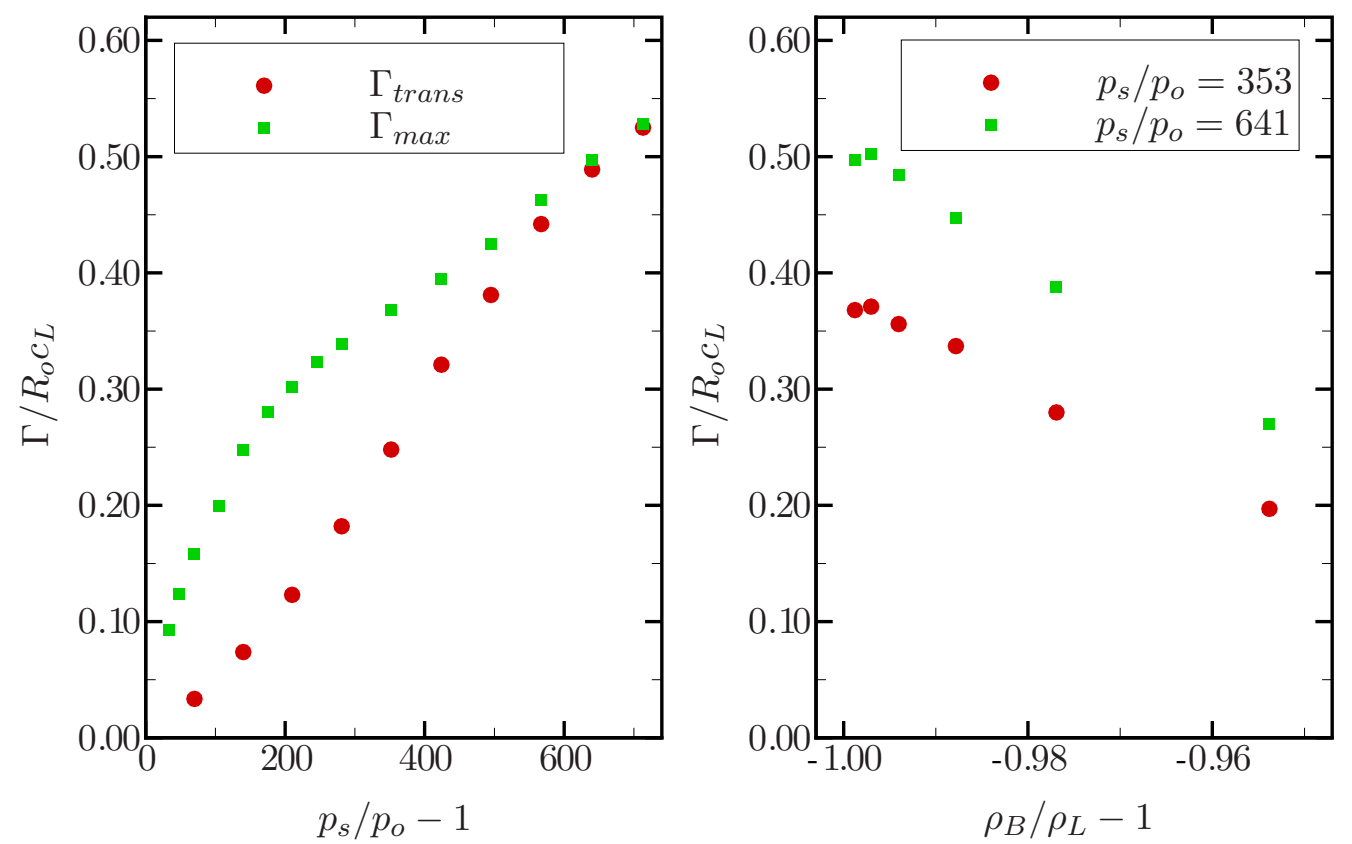

Figure 4.28: Circulation after the propagation of the internal shock through the entire bubble in free-field shock-induced collapse. Left: dependence on the pressure ratio across the shock; right: dependence on the density ratio across the interface.

\subsection{Jet formation mechanism in shock-induced collapse}

Based on the results presented in the previous section, jet formation in shockinduced collapse is discussed qualitatively by considering several mechanisms that have been proposed previously (e.g., flow induced by the passage of the shock, non-spherical perturbations and baroclinic torque) and a new one (the transient nature of shock propagation). Observations on jet formation in Rayleigh collapse near a wall are provided at the end of the section. 


\subsubsection{Flow induced by the passage of the shockwave}

In studies of ignition and propagation of explosive reactions, Dear \& Field (1988) and Bourne \& Field (1992) considered the shock-induced collapse of two-dimensional cavities in a water/gelatin matrix. Linearized shock dynamics relations are employed to show that the speed of the proximal interface increases in such a way that a jet forms. Using self-similarity and linearized relations, Tulin (1969) showed that jets may form upon the impingement of weak shockwaves on curved interfaces. Ohl \& Ikink (2003) further remarked that the flow induced by the shock would emphasize the jetting phenomenon.

The initial motion of the interface can be treated analytically. As noted by Haas \& Sturtevant (1987), as the shock passes over a fluid inhomogeneity, the shape of the volume changes due to compression and shear. In the simplest configuration of a homogeneous medium, a circle of diameter, $D$, deforms into an ellipse of major axis, $D$, and minor axis, $D\left(1-u_{p} / v_{s}\right)$, where $v_{s}$ and $u_{p}$ are the velocities of the shock and of the fluid induced by the shock. However, if the fluids are different, oblique interactions lead to shear, as exemplified by the baroclinic vorticity generated along the interface described in Section 4.3.9.

The initial velocity of the interface just after the interaction with the shock can be calculated by extending the analysis of Section 4.3.2 and considering the refraction of an oblique shock at an interface between two different fluids (Henderson, 1989). The initial velocity at $r=0$ is completely radial with respect to the bubble, whereas it is completely tangential at the top of the bubble. In between, the direction of the velocity vector with respect to the bubble wall changes smoothly, so that the interface velocity is largest at $r=0$. From Equation 4.28 and using basic geometry, the radial velocity imparted by the passage of the shock to the bubble is given by Dear \& Field (1988): $v_{f}=2 u_{p} \cos \theta$, where $u_{p}$ is the particle velocity behind the shock and $\theta$ is the angle with respect to the horizontal ( $x$-axis) in the present configuration. The bubble shape (in two dimensions) resulting from such a velocity condition is specified along the interface can be computed: as the shock interacts with a given point on the interface, this point moves with a constant 
velocity with components, $\left(v_{f} \cos \theta, v_{f} \sin \theta\right)$, where $\theta$ is the angle with the horizontal. Figure 4.29 shows results for a large pressure ratio across the shock (Dear $\&$ Field, 1988) and for two pressure ratios representative of the present work. The initial shape and the shape at a time equal to the collapse time for the relevant pressure ratio are included.
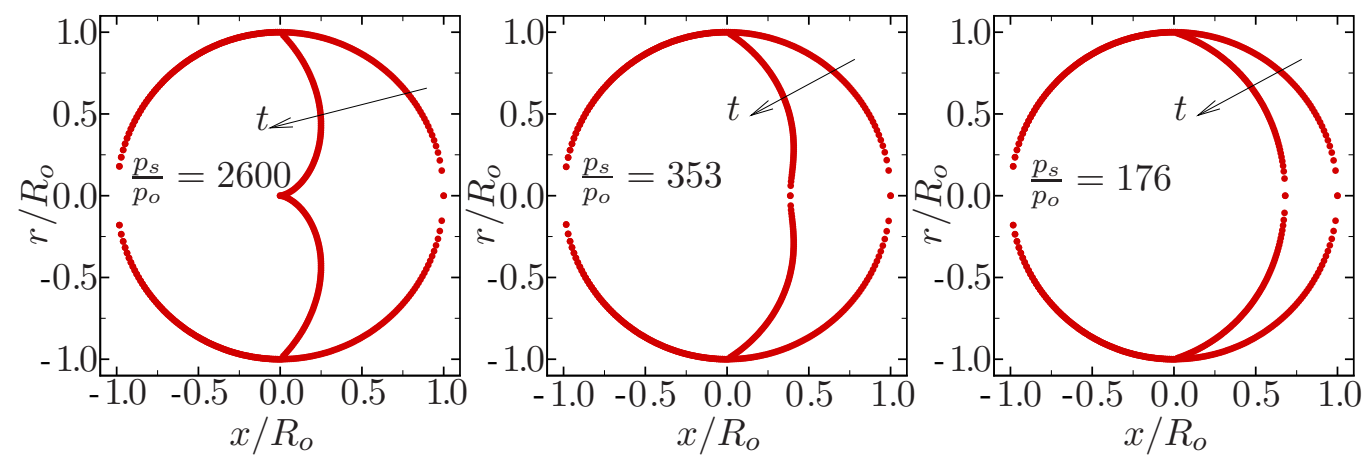

Figure 4.29: Collapse of a bubble based on the flow induced by the passage of the shock. Left: $u_{p}=-0.1, t=0,5$ (Dear \& Field, 1988); middle: $u_{p}=-0.027$, $t=0,7.98$; right: $u_{p}=-0.014, t=0,11.3$.

In the problem considered by Dear \& Field (1988), a jet forms in a finite time for an initial shockwave of $0.26 \mathrm{GPa}^{8}$ due to the flow imparted by the shockwave as it refracts through the bubble, as shown in the left frame of Figure 4.29. However, no comparison of the time scales between the theory and the experiment are provided. The non-dimensional time it takes for the jet to travel to the bubble center is given based on the normal shock relations in the liquid:

$$
t_{d}=\frac{1}{2 u_{p}}=\frac{1}{2} \frac{\sqrt{\frac{\gamma+1}{2}\left(\frac{p_{s}}{p_{o}}-1\right) \frac{p_{o}}{\rho_{L} c_{L}^{2}}+1}}{\left(\frac{p_{s}}{p_{o}}-1\right) \frac{p_{o}}{\rho_{L} c_{L}^{2}}}
$$

This characteristic formation time is to be compared to the collapse time of a bubble, which is $\tau_{c} \approx 1 / \sqrt{\left(p_{s} / p_{o}-1\right) p_{o} / \rho_{L} c_{L}^{2}}$. If the bubble had been spherical in the experiments, the collapse time corresponding to this pressure ratio would be

\footnotetext{
${ }^{8}$ It is not clear that the weak shock approximations hold for such a pressure ratio.
} 
$\tau_{c} \approx 3$, so that the proposed mechanism for jet formation would be relevant for the pressure ratio considered by Dear \& Field (1988). In the range of pressure ratios considered in this work, $u_{p}$ is much smaller, so that the jet does not have time to develop before collapse, as shown in the middle and right frames of Figure 4.29. Thus, this mechanism alone is not sufficient to be responsible for jet formation under the current conditions.

\subsubsection{Non-spherical perturbations}

As illustrated in the previous section, the passage of the shockwave perturbs the bubble shape and velocity. Thus, the non-spherical perturbation analysis discussed in Section 4.1.4 may be relevant to jet formation. After the passage of the shock, the bubble is no longer strictly spherical. This shape can be described using modes $n=0,1,2$ in Equation $4.25 .^{9}$ In particular, the initial perturbations, $a_{0}(0)=$ $-0.010, a_{1}(0)=-0.027$, and $a_{2}(0)=-0.018$, represent the bubble interface very well after just after the passage of the shock. In addition, the initial velocity of the translational mode is non-zero: $\dot{a}_{1}(0)=u_{C D}=-0.027$. Equation 4.26 can then be integrated numerically in time until collapse. The history of the amplitudes are shown in Figure 4.30, along with the initial perturbed bubble shape (just after the passage of the shock, $t=1.95)$ and the shape at collapse $(t=8.95)$.

The amplitude of modes 0 and 2 does not grow significantly until the last stages of collapse, while that of mode 1 grows from the beginning, due to the non-zero velocity perturbation. ${ }^{10}$ Mode 2 first becomes positive, leading to a deformation in the direction opposite to the jet. This is due to the fact that the initial perturbation first compresses the bubble, so that it relaxes during collapse. Although a jet is starting to develop, this occurs too late in the collapse to be the sole mechanism responsible for jet formation.

\footnotetext{
${ }^{9}$ Mode 0 corresponds to a volumetric perturbation, mode 1 to a translational perturbation (and is constant, from Equation 4.26), and mode 2 to a spheroid perturbation.

${ }^{10}$ If the perturbation velocity of mode 1 had been zero, $a_{1}(t)=0$ and the $j e t$ would not have formed.
} 

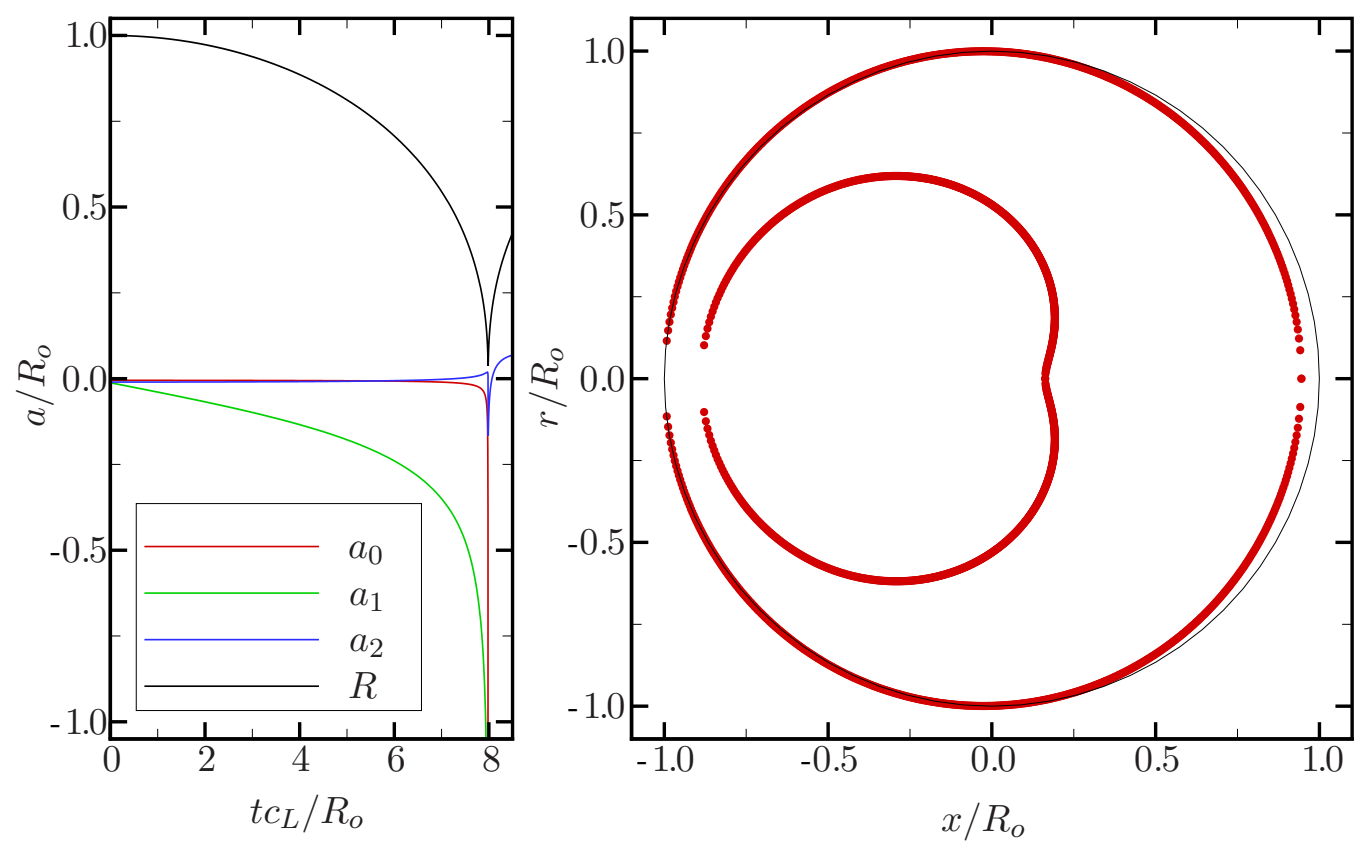

Figure 4.30: Non-spherical perturbations of the bubble interface. Left: history of the amplitude of the perturbations; right: initial perturbation and perturbation at collapse $\left(t c_{L} / R_{o}=1.95\right.$ and $t c_{L} / R_{o}=8.95$ for shock-induced collapse).

The present results however are not strictly applicable, because the amplitude of the perturbations is not small for very long. In particular in the case $n=1, a_{1}$ is already not much smaller than $R$ early in the collapse. Thus, nonlinear effects are no longer negligible and may therefore play a role in the jet formation.

\subsubsection{Flow induced by baroclinic vorticity}

In studies of shock propagation in non-uniform media, Haas \& Sturtevant (1987) conducted experiments of shockwaves in gases interacting with gaseous cylinders and concluded that the baroclinic vorticity generated by the passage of the shockwave over the bubble was the mechanism responsible for jet formation. Haas \& Sturtevant (1987) and later simulations of Picone \& Boris (1988) and Quirk \& Karni (1996) showed that the configuration of the problem led to qualitatively different behavior, depending on whether the gas within the cylinder is heav- 
ier/lighter. If the impedance of the bubble is lower, then the usual jet forms in the direction of propagation of the shockwave and the bubble becomes a vortex ring. In the inverse configuration, the bubble eventually takes the form of a pair of vortex lines, with no obvious jet. This latter case has been observed in the experiments on shock-droplet interactions of Joseph et al. (1999).

The effect of baroclinic vorticity can be assessed by adapting the RichtmyerMeshkov instability analysis of Haas \& Sturtevant (1987) for shock-bubble interactions. The main difference lies in the fact that the volumetric changes are substantial in the present case. Thus, the base flow is assumed to consist of a spherically collapsing bubble, which is provided by the simulation of Rayleigh collapse. In other words, the interfacial velocity difference at the jet location between shockinduced collapse and Rayleigh collapse, $v_{j, S I C}-v_{j, R C}$, is expected to be equal to the perturbation velocity obtained in a Richtmyer-Meshkov analysis. The bubble can be considered to be a perturbation of amplitude, $\eta=R_{o}$, and of wavelength, $\lambda=2 R_{o}$. The wavenumber for a sphere is $k=\sqrt{2} / R_{o}$, so that the perturbation velocity at the jet location is:

$$
v=k \eta V A,
$$

where $V$ is the change in translational velocity due to the passage of the shock (for the corresponding one-dimensional Riemann problem) and

$$
A=\frac{\rho_{2}-\rho_{1}}{\rho_{2}+\rho_{1}}
$$

is the Atwood number. In the present case, the base flow is the Rayleigh collapse, so that $V=u_{C D, S I C}-u_{C D, R C}$, where $u_{C D}$ is calculated as in Section 4.3.2. For $p_{s} / p_{o}=353$ and for an air bubble in water, $A=-0.998$, so that $v \approx-\sqrt{2}\left(u_{C D, S I C}-u_{C D, R C}\right)=-1.81 \times 10^{-2}$, using the measurements from Section 4.3.2. From Figure 4.31, the velocity difference is $v \approx-1.65 \times 10^{-2}$, thus showing reasonable agreement. The same calculation was carried out for different Atwood numbers and is shown in Table 4.2. Again, the perturbed velocity is calculated from Equation 4.33 using the velocity difference from the initial Riemann 
problem in Rayleigh collapse and shock-induced collapse, while the measured velocity is the difference in the simulations between the jet velocity and the velocity of the distal side. The measurements are difficult to perform because the data is recorded at discrete times and must therefore be interpolated. Nevertheless, the agreement between the theory and the computations is good. Thus, the present analysis shows that baroclinic effects add a net velocity in the direction of propagation of the jet. However, this mechanism is not sufficient to be the lone cause of jet formation.

Table 4.2: Perturbation velocity due to baroclinic vorticity for shock-induced collapse $\left(p_{s} / p_{o}=353\right)$.

\begin{tabular}{c|cc}
$A$ & $v / c_{L}($ Eq. 4.33$)$ & $v / c_{L}$ (measured) \\
\hline-0.998 & $-1.81 \times 10^{-2}$ & $-1.65 \times 10^{-2}$ \\
-0.952 & $-1.73 \times 10^{-2}$ & $-1.40 \times 10^{-2}$ \\
-0.885 & $-1.60 \times 10^{-2}$ & $-1.28 \times 10^{-2}$ \\
-0.782 & $-1.41 \times 10^{-2}$ & $-1.10 \times 10^{-2}$ \\
-0.607 & $-1.09 \times 10^{-2}$ & $-9.50 \times 10^{-3}$
\end{tabular}

\subsubsection{Transient nature of shock propagation}

In the previous three sections, it is shown that the effects of the flow induced by the passage of the shock, the baroclinic vorticity and the non-spherical perturbations alone are not sufficient to generate a re-entrant jet in shock-induced collapse. However, these effects ignore the fact that the passage of the shock over the bubble is a transient process is ignored: the proximal side is subjected to the pressure increase sooner than the distal side, because of the finite speed of propagation of the shock. Thus, the proximal portion of the bubble is expected to contract under the effect of the shock before the distal side does. Though the sound speed in water is often assumed infinite, it is approximately only five times larger than that in air. In certain cases, the time it takes for the external shock to propagate around the 
bubble is almost half of the collapse time, as shown in Section 4.3.1.

In order to provide a qualitative understanding of the process, the velocity of the jet and distal side for $p_{s} / p_{o}=353$ are shown in Figure 4.31. The top plot shows the history of the velocity of the jet and distal side for shock-induced collapse. The middle plot includes results for Rayleigh collapse and for the Rayleigh-Plesset equation as well; in shock-induced collapse, $t=0$ is taken to be the time when the shock first hits the bubble for the jet velocity and when the shock diffracts to $r=0$ for the velocity of the distal side. ${ }^{11}$ The bottom plot shows the bubble non-sphericity. The corresponding phase diagram, which displays the velocity of the proximal side with respect to its position, is shown in Figure 4.32.

The top plot of Figure 4.31 shows that the proximal side follows the slow regime described in Section 4.1.1; on the other hand, the distal side only starts its motion at $t \approx 4.5$. The proximal side enters the fast regime at $t \approx 7$ and undergoes significant acceleration, while the distal side is still in the slow regime when the jet impacts it.

By comparing the trajectories of shock-induced collapse to those of Rayleigh collapse in the middle plot of Figure 4.31, it is clear that the distal side follows the motion of spherical collapse driven by a $p_{s} / p_{o}=353$ pressure ratio. However, because of the delay due to the finite speed of propagation of the shock in water, the distal side is still in its slow regime when the jet impacts it.

Furthermore, the velocity of the proximal side (jet) is slightly higher in shockinduced collapse than in Rayleigh collapse. After $t \approx 3$ in the middle plot, the acceleration is nearly the same in both cases, so that there is an approximately constant velocity difference between the two cases, until $t \approx 5$. This effect is attributed to the baroclinic vorticity, as discussed in Section 4.4.3. Thus, the higher velocity allows the proximal side to enter the fast regime sooner. As illustrated by the bottom plot of Figure 4.31, the bubble starts to becomes non-spherical as it enters the fast regime in shock-induced collapse. ${ }^{12}$ In the fast regime, the

\footnotetext{
${ }^{11}$ In both cases, the impulse due to the initial Riemann problem has been subtracted, so that both curves start at $v=0$.

${ }^{12}$ Then, as discussed in Section 4.3.3, the interface becomes Rayleigh-Taylor unstable even in
} 

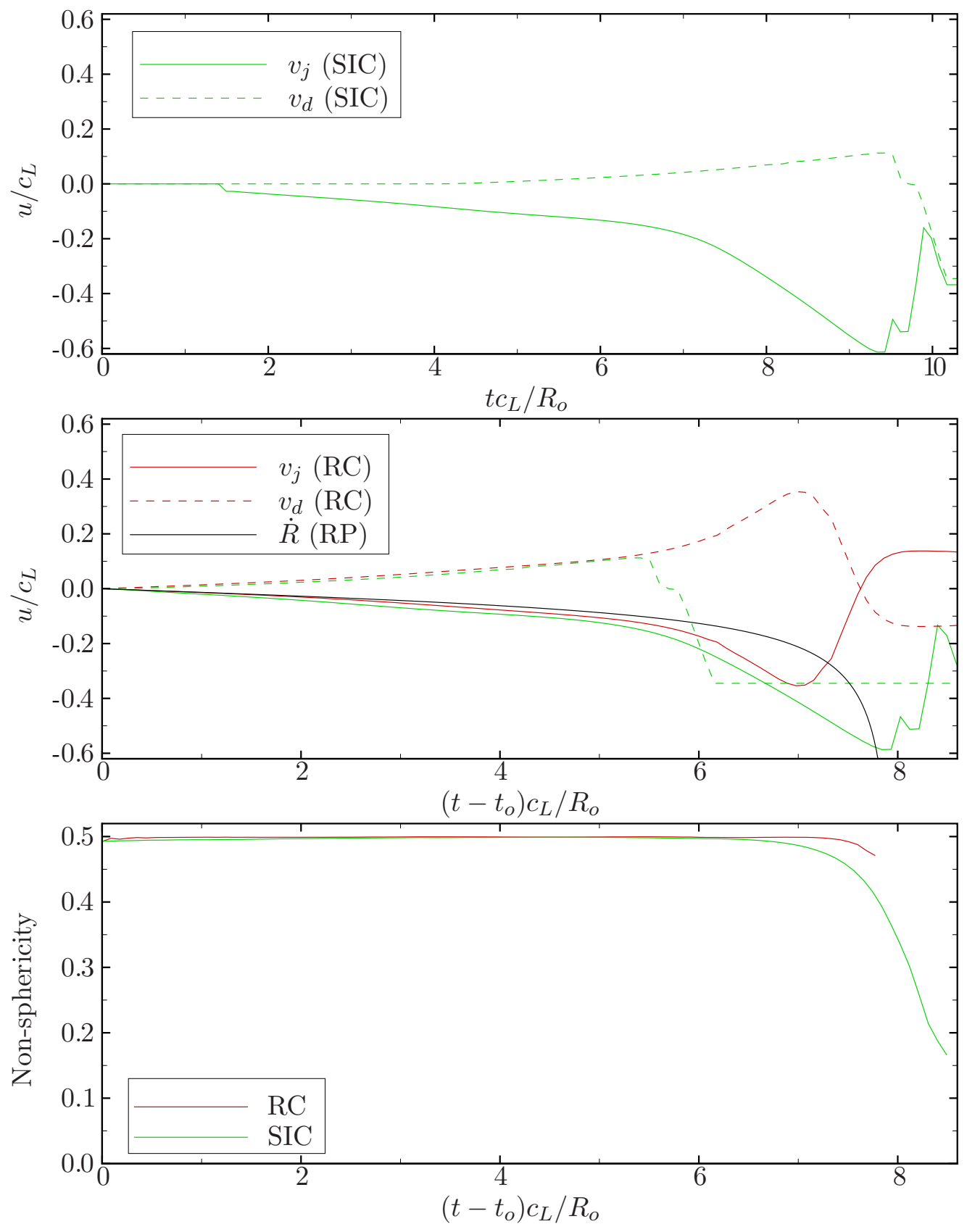

Figure 4.31: Jet velocity and bubble non-sphericity in jet formation for shockinduced collapse $\left(p_{s} / p_{o}=353\right)$. Top: velocity of the jet and distal side for shock-induced collapse. Middle: velocity of the jet and distal side for Rayleigh collapse, shock-induced collapse (the plot was adjusted to have the same origin as for Rayleigh collapse) and the Rayleigh-Plesset equation. Bottom: bubble nonsphericity. 


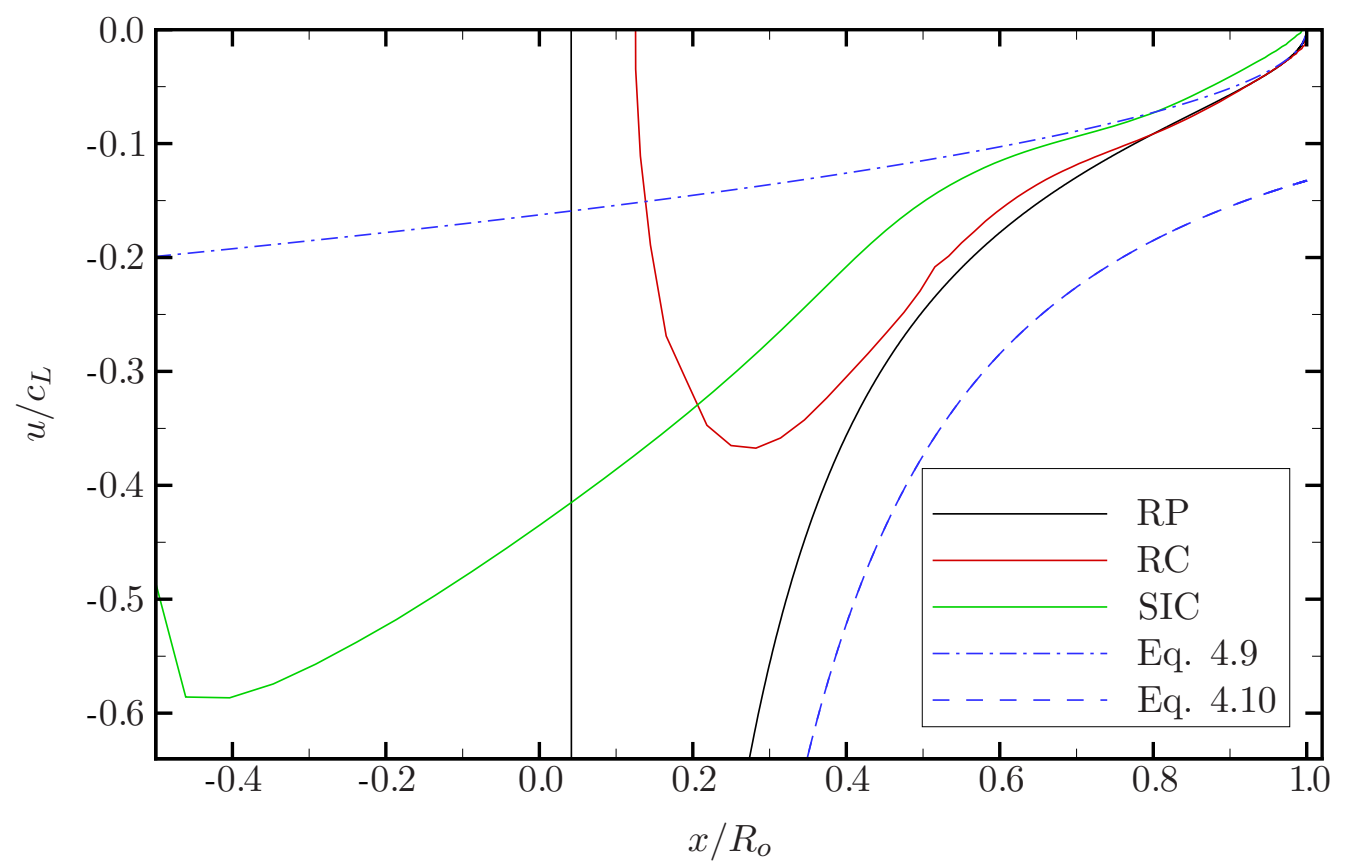

Figure 4.32: Jet velocity as a function of the interface position for free-field shockinduced collapse $\left(p_{s} / p_{o}=353\right)$.

acceleration is higher in Rayleigh collapse, as observed in the phase diagram of Figure 4.32, because the collapse is spherical.

The reason that the velocity of the proximal side (jet velocity) is higher in shock-induced collapse is now clear. While the distal side is contracting more slowly, the proximal side keeps on accelerating in the fast regime and can therefore penetrate the bubble to a greater amount, until impact with the distal side. In the spherical case, the collapse is arrested sooner, because the trajectory of the distal side is the exact opposite of that of the proximal side.

The delay in the contraction of the distal side is a direct consequence of the transient nature of shock propagation; because the proximal side starts to contract sooner than the distal side, it enters the fast regime much sooner. In light of this observation, the previous findings that each of the effects considered previously (flow induced by the passage of the shock, non-spherical perturbations and baroRayleigh collapse. 
clinic vorticity) adds a net velocity to the proximal side, allows the fast regime to be achieved sooner. Though this analysis still does not paint the full picture of jet formation, it sheds additional light on the matter.

\subsubsection{Rayleigh collapse near a wall}

In Rayleigh collapse near a wall, based on potential theory, Plesset \& Prosperetti (1977) argued that the interfacial velocity farthest from the wall must be highest during collapse, because of the flow induced by the image bubble. However, this effect is minor. Following the ideas of Benjamin \& Ellis (1966), Vogel et al. (1989) explain that a pressure gradient across the bubble is created by the retardation of the flow because of the solid boundary. In order to preserve its Kelvin impulse, the bubble accelerates towards the wall as it collapses, so that the velocity of the interface farthest from the wall must grow much faster than that of the opposite side, thus leading to the formation of a jet. Philipp \& Lauterborn (1998) extend this explanation by remarking that the fluid volume above the bubble is accelerated and focused during collapse. From boundary integral simulations, Best \& Kucera (1992) remark that a high pressure is computed behind the bubble increases during collapse due to the acceleration towards the wall, and that this pressure drives the jet into the bubble. However, Section 4.1.2 shows that this peak pressure is a consequence of the acceleration of the bubble interface, not a cause for it. This high pressure behind the bubble has also been observed in experiment (Ward \& Emmony, 1991).

The ideas developed in the previous section are used to better understand jet formation in Rayleigh collapse near a wall. Figure 4.33 shows the history of the velocity of the jet and distal side, and of the bubble non-sphericity, for Rayleigh collapse near a wall with $p_{s} / p_{o}=353$ and $H_{o} / R_{o}=1.2,1.5,2.0,5.0$. The solution to the Rayleigh-Plesset equation for spherical collapse with the same initial pressure ratio is also provided.

The history of the jet velocity follows the same behavior for the different standoff distances until the collapse time of the farthest bubble. This indicates that the 

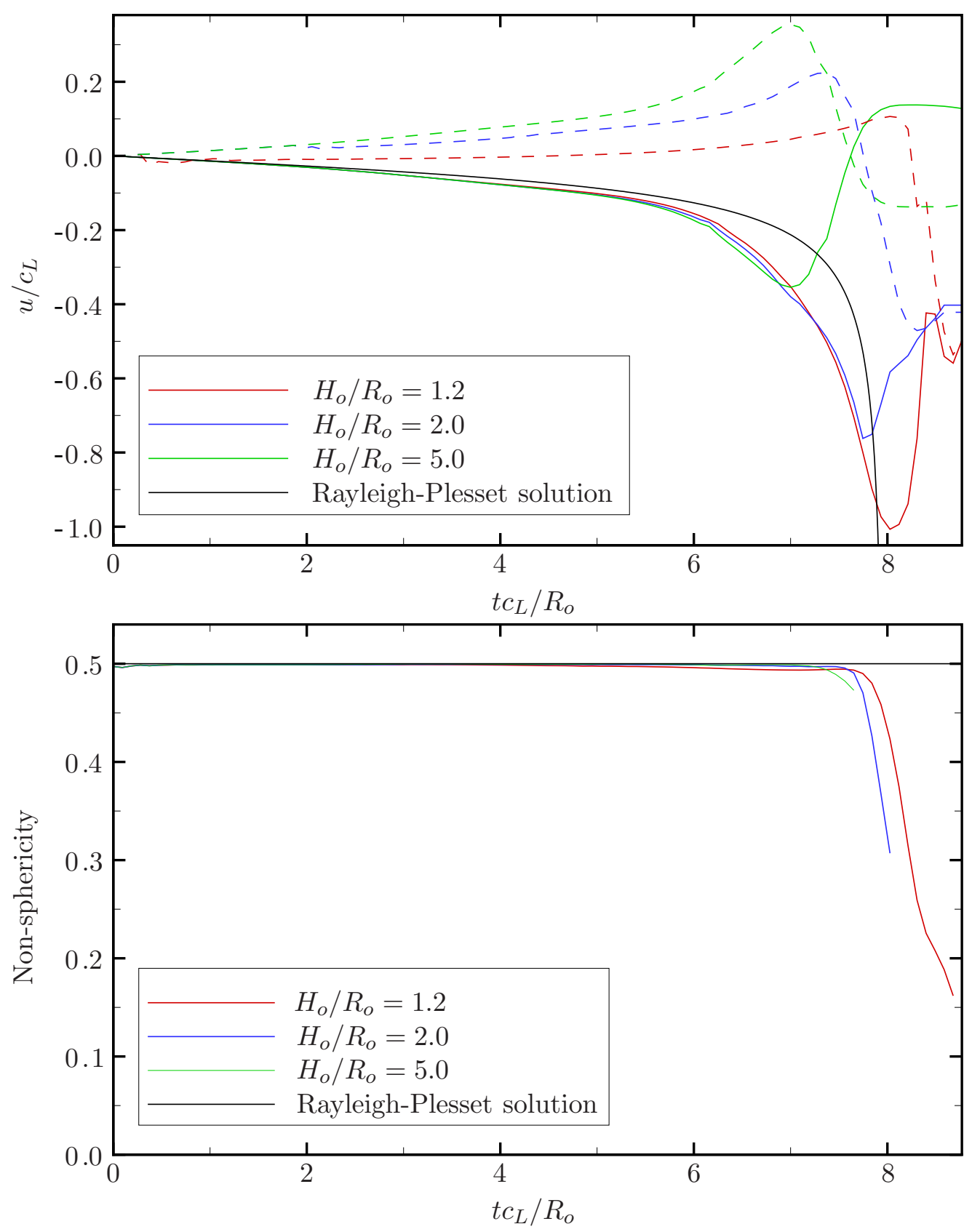

Figure 4.33: Speed of the jet and distal side (top) and bubble non-sphericity (bottom) for Rayleigh collapse near a wall $\left(p_{s} / p_{o}=353, H_{o} / R_{o}=1.5\right)$.

wall does not significantly affect the flow on the proximal side. Deviations occur only in the late stages of collapse. On the other hand, the velocity of the distal 
side (closest to the wall) is very different because the wall retards the flow. In the present compressible framework, the presence of the wall is communicated to the bubble by the reflection of the initial expansion wave back onto the bubble. As a result, the pressure driving the bubble collapse decreases as the expansion propagates over the bubble. This phenomenon would also occur in incompressible simulations (Plesset \& Chapman, 1971; Blake et al., 1986; Zhang et al., 1993), where it would happen instantaneously because the sound speed is effectively infinite in such cases. The strength of the expansion wave decreases with distance, so that this effect is not as severe on the proximal side. Thus, the pressure driving the collapse on the proximal side is essentially unchanged, while that driving the distal side is reduced. Hence, the contraction of the proximal side approximately follows the behavior of free-field collapse, while that of the distal side is lessened because of the reduced local pressure. The proximal side then enters the fast regime much sooner than the distal side. Since the distal side is contracting much more slowly, the proximal side can propagate farther because nothing arrests its motion, thus forming the jet. Furthermore, as noticed by Vogel et al. (1989), parts of a bubble with a higher curvature collapse faster, thus providing another mechanism that leads to a higher jet velocity.

\subsection{Damage potential of single-bubble collapse}

For the initial stand-off distances considered in the present work, the potential for damage of bubble collapse is related to the shockwaves that are emitted during collapse. First, these shockwaves are characterized. Then, the water-hammer pressure and the radiated energy are investigated. Next, measurements for the wall pressure are provided. Finally, the dependence on the shock amplitude is studied and the shielding effects of the bubble are considered. 


\subsubsection{Shock emission}

In the present simulations, the emission of three main types of shockwaves are observed during bubble collapse:

1. A precursor shockwave generated inside the bubble by the piston-like motion of the jet,

2. A gas compression shockwave due to the compression of the gas inside the bubble, and

3. A water-hammer shockwave caused by the impact of the jet onto the distal side.

In experiments of Rayleigh collapse near a wall and shock-induced collapse (Ohl et al., 1999; Lindau \& Lauterborn, 2003), the water-hammer and gas compression shockwaves are observed, though Sankin et al. (2005) notes that, if the camera speed is not fast enough, the shocks will have merged into one front by the time it is visualized. Ohl et al. (1999) further postulate the generation of a "tip-bubble shockwave" caused by the compression of a gas pocket between the jet tip and the distal side; however, Lindau \& Lauterborn (2003) argue that this feature is simply part of the water-hammer shock.

In order to visualize the sequence of shockwave emission, line plots of pressure, axial velocity, $\gamma$ and density along the axis, $r=0$, and the corresponding contours are shown. In the line plots, the first line is black, the second is red, the third is blue and the fourth is green. Only a small part of the domain is considered and the scales of the different plots of the same quantity are different in order to highlight the important features.

\section{Free-field Rayleigh collapse}

First, free-field Rayleigh collapse with $p_{s} / p_{o}=353$ is considered to understand the initial wave dynamics and how the gas compression shockwave is produced. 

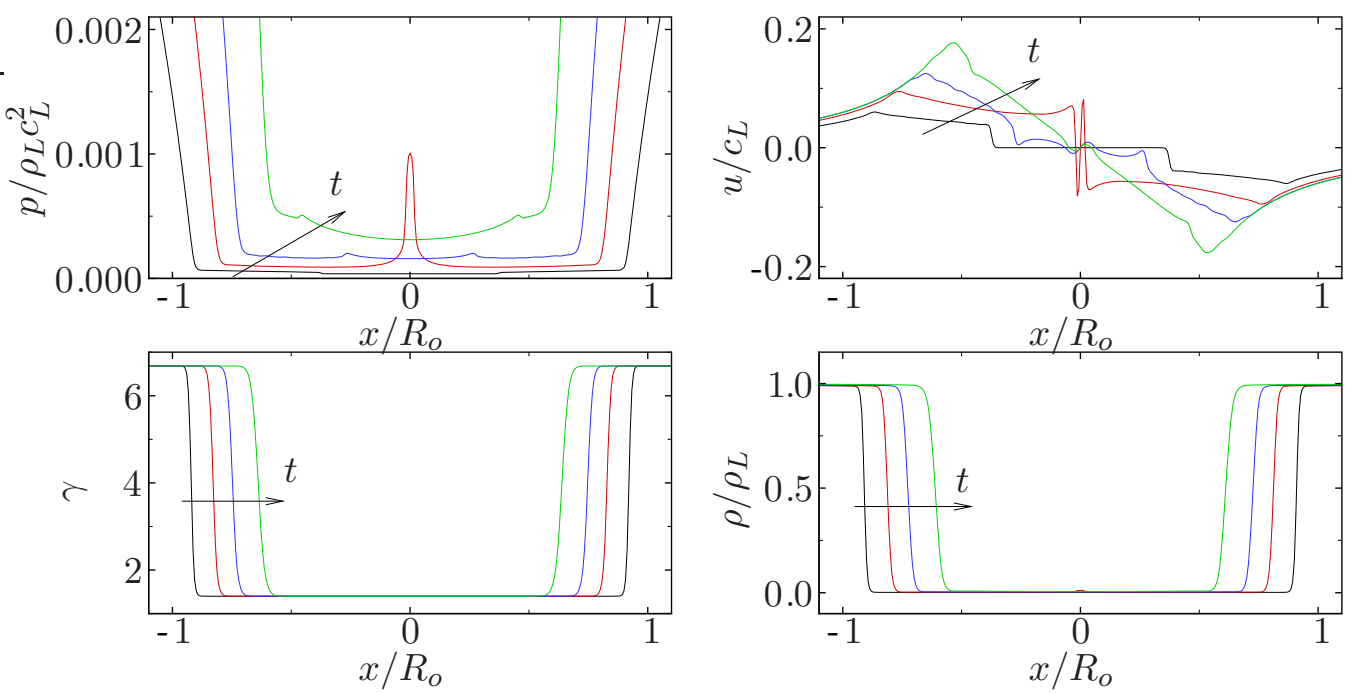

Pressure

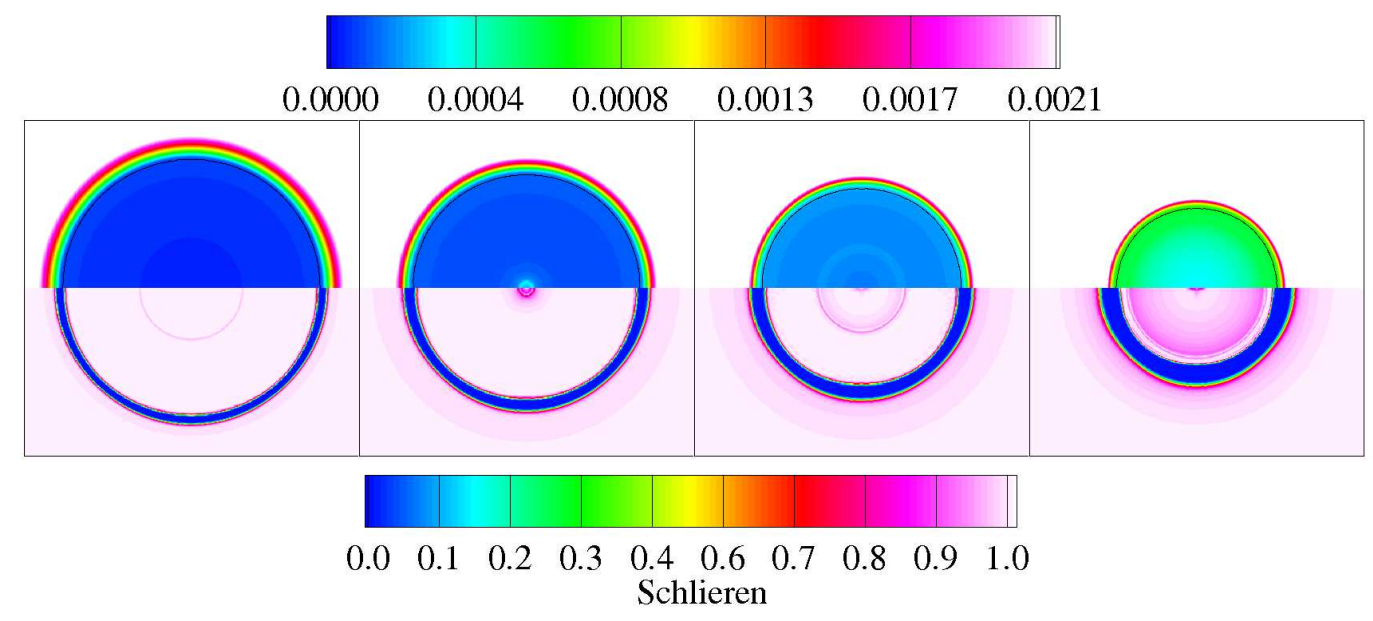

Figure 4.34: Initial flow field for free-field Rayleigh collapse $\left(p_{s} / p_{o}=353\right)$ at $t c_{L} / R_{O}=2.84,4.26,5.15,6.04$. Top: pressure, velocity, density, and $\gamma$ profiles along $r=0$; bottom: pressure and numerical Schlieren contours.

Figure 4.34 shows the initial behavior of the problem at $t c_{L} / R_{o}=2.84,4.26$, $5.15,6.04$. As discussed in Section 4.3.2, the shockwave released by the initial Riemann problem at the interface is weak. While propagating to the center of the bubble, it focuses until it converges at the origin, thereby achieving a relatively high pressure. The wave then propagates back outwards and reflects off the interface, which is moving radially inwards due to the collapsing bubble. Because 
of the high impedance mismatch, the shock is reflected almost perfectly. These reflections occur multiple times and do not significantly affect the dynamics of collapse initially.
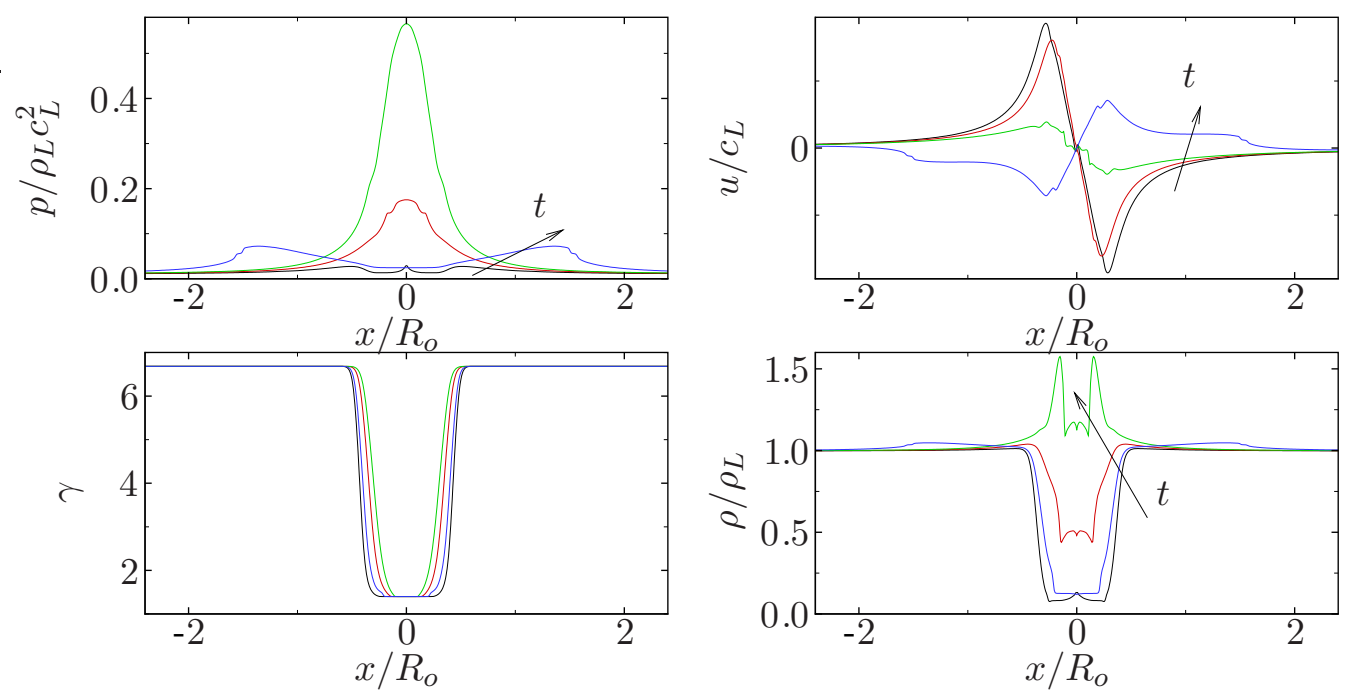

Pressure

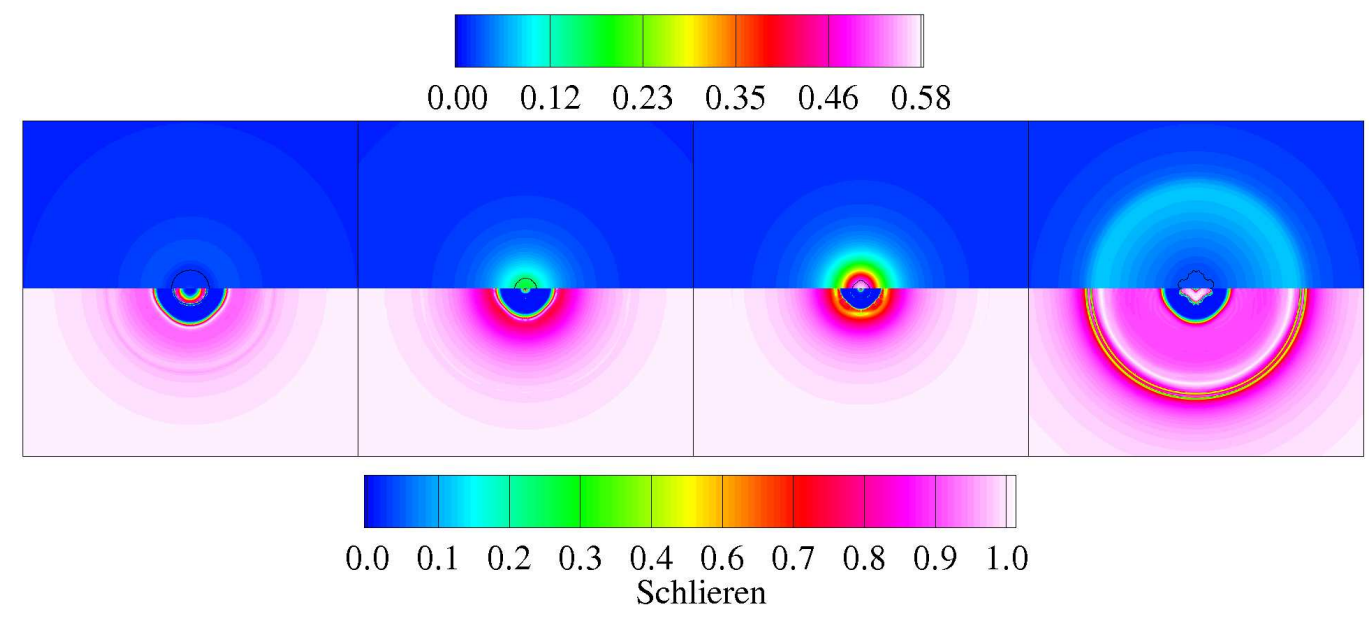

Figure 4.35: Flow field for Rayleigh collapse $\left(p_{s} / p_{o}=353\right)$ just before and after collapse at $t c_{L} / R_{o}=7.10,7.46,7.72,8.79$. Top: pressure, velocity, density, and $\gamma$ profiles along $r=0$; bottom: pressure and numerical Schlieren contours.

Figure 4.35 depicts the behavior at $t c_{L} / R_{o}=7.10,7.46,7.72,8.79$, just before and after collapse. As discussed in Section 4.1.2, the pressure behind the interface increases to a value higher than $p_{\infty}$ when the bubble pressure and the interface 
velocity reach a critical value; this is difficult to see in Figure 4.35 because of the scale. The pressure due to the intersection of the internal shock is now more substantial within the bubble. The kinks in the pressure profiles are caused by the reflection of the shockwave trapped within the bubble. The local interface velocity decreases due to the compression of the gas before the bubble reaches its minimum volume. At this stage, the gas compression shock is emitted and propagates in the liquid. The bubble expands and a shockwave propagates radially outwards. The bubble contents remain at a high pressure and density in the initial stages of rebound. The bubble interface undergoes a large change in acceleration at collapse, so that its shape becomes distorted. As described in Section 4.5.4, the amplitude of the shock front decays as $1 / r$.

\section{Rayleigh collapse near a wall}

Rayleigh collapse near a wall with $H_{o} / R_{o}=1.5$ and $p_{s} / p_{o}=714$ is considered to illustrate the emission of the precursor and water-hammer shockwaves.

Figure 4.36 presents the results at $t c_{L} / R_{O}=5.04,5.17,5.30,5.43$, just before the water-hammer. Inside the bubble, the shock released by the initial Riemann problem at the interface is trapped and reflects internally. Because the bubble walls are moving inwards, the amplitude of the shock increases with each reflection. As the bubble collapses, the jet speed and the pressure behind the proximal side increases according to the phenomenon described in Section 4.1.2. This feature has been observed in prior experiments (Ward \& Emmony, 1991) and simulations (Blake et al., 1986; Best \& Kucera, 1992; Zhang et al., 1993). It was even postulated by Best \& Kucera (1992) that this high pressure drives the jet into the bubble. However, as noted in Section 4.1.2, this high pressure is a consequence of the increasing interface speed, not a cause for it. The motion of the jet causes the gas on the proximal side of the bubble to compress, ultimately leading to the formation of a left-moving shockwave (the precursor shock) due wave steepening (at $x / R_{o} \approx 2.9$ on the green line at $t=5.43$ ). During this process, the jet acts like an advancing piston. However, unlike the predictions of Benjamin (1958), this 

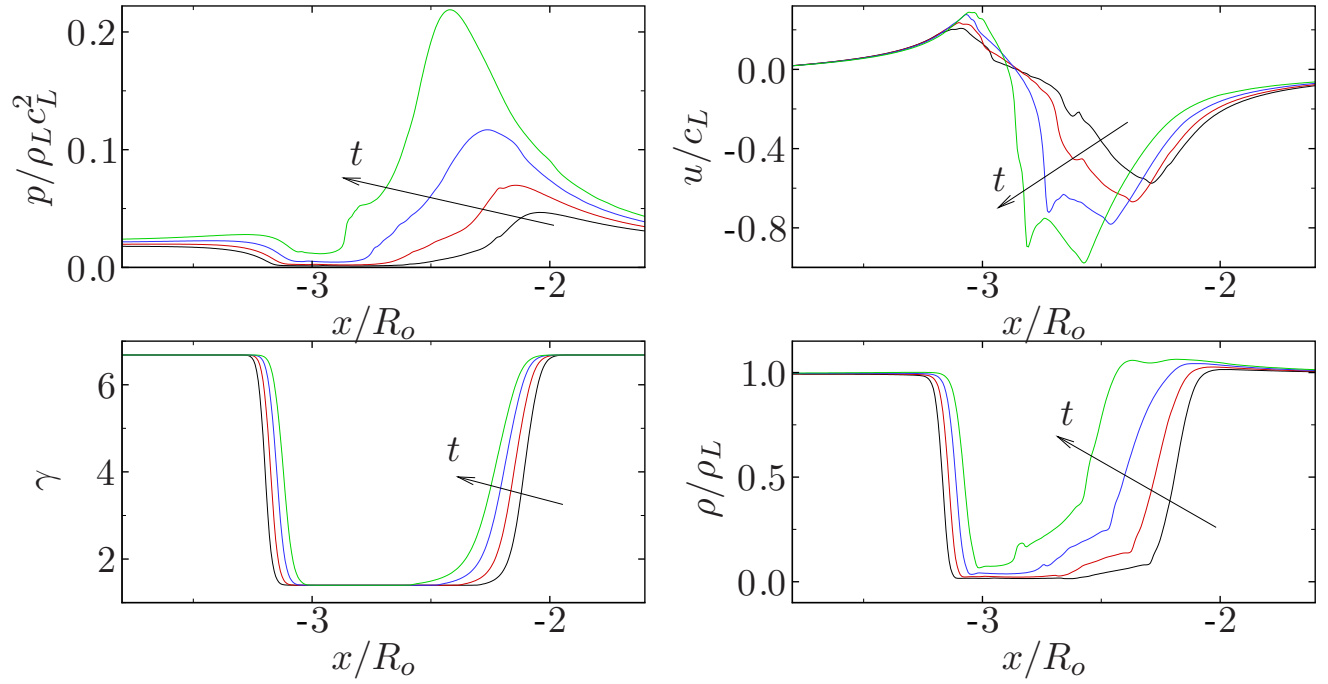

Pressure

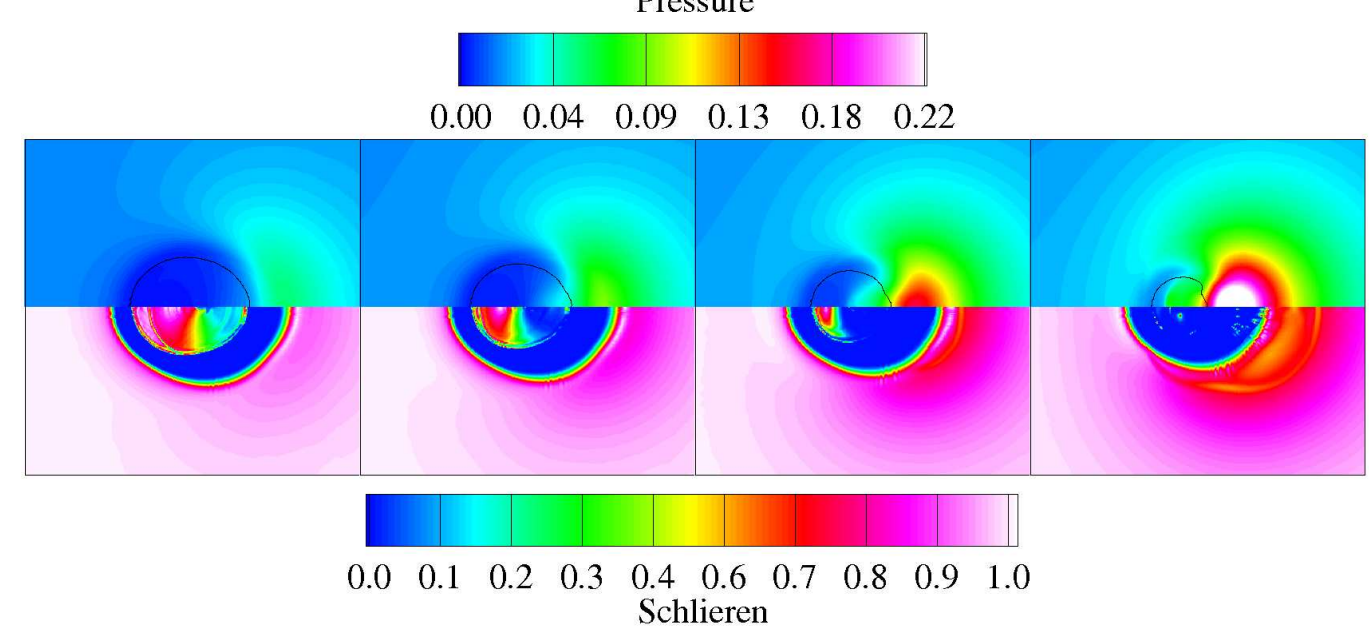

Figure 4.36: Flow field just before the impact of the jet onto the distal side for Rayleigh collapse near a wall $\left(p_{s} / p_{o}=714, H_{o} / R_{o}=1.5\right)$ at $t c_{L} / R_{o}=5.04,5.17$, 5.30, 5.43. Top: pressure, velocity, density, and $\gamma$ profiles along $r=0$; bottom: pressure and numerical Schlieren contours.

phenomenon clearly occurs inside the bubble. The resulting shock may be the one observed in the experiments of Ohl et al. (1999) and Lindau \& Lauterborn (2003).

Figure 4.37 presents the results at $t c_{L} / R_{o}=5.56,5.69,5.82,5.95$ just after the water-hammer. The precursor shock is seen exiting the bubble. When the jet hits the distal side, a sharp pressure discontinuity is produced. At this stage, 

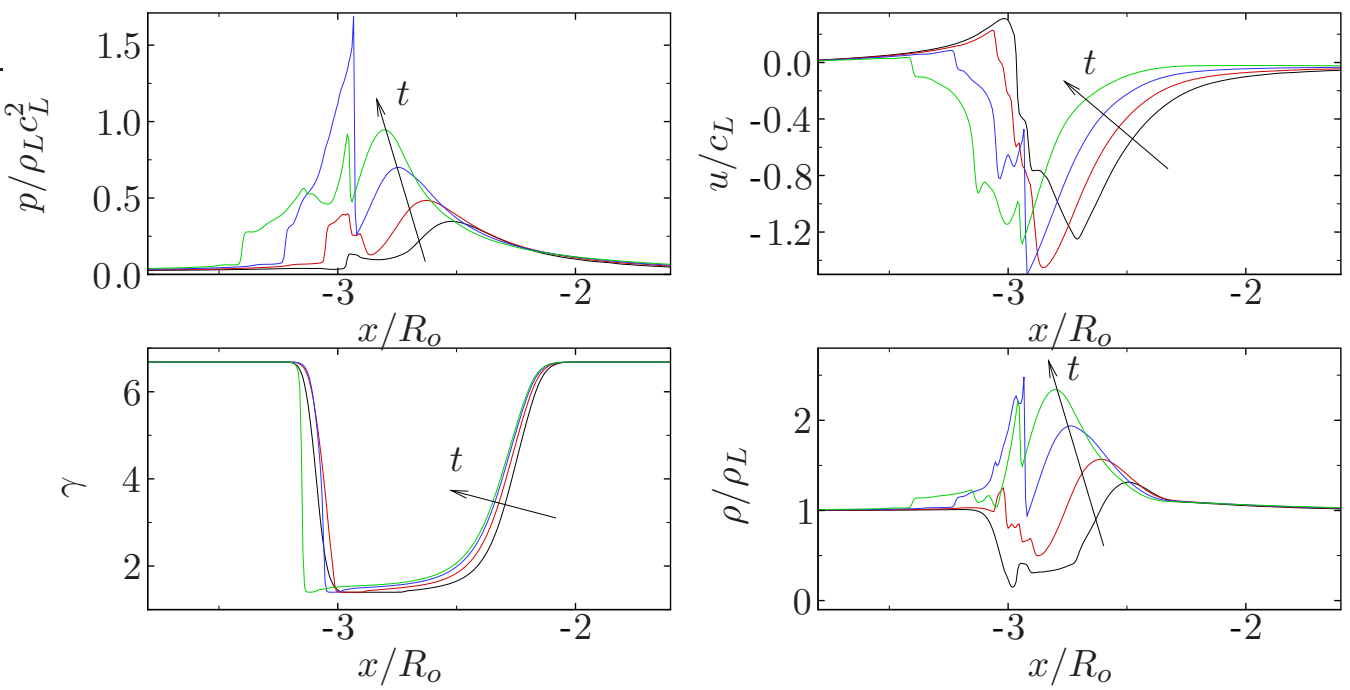

Pressure

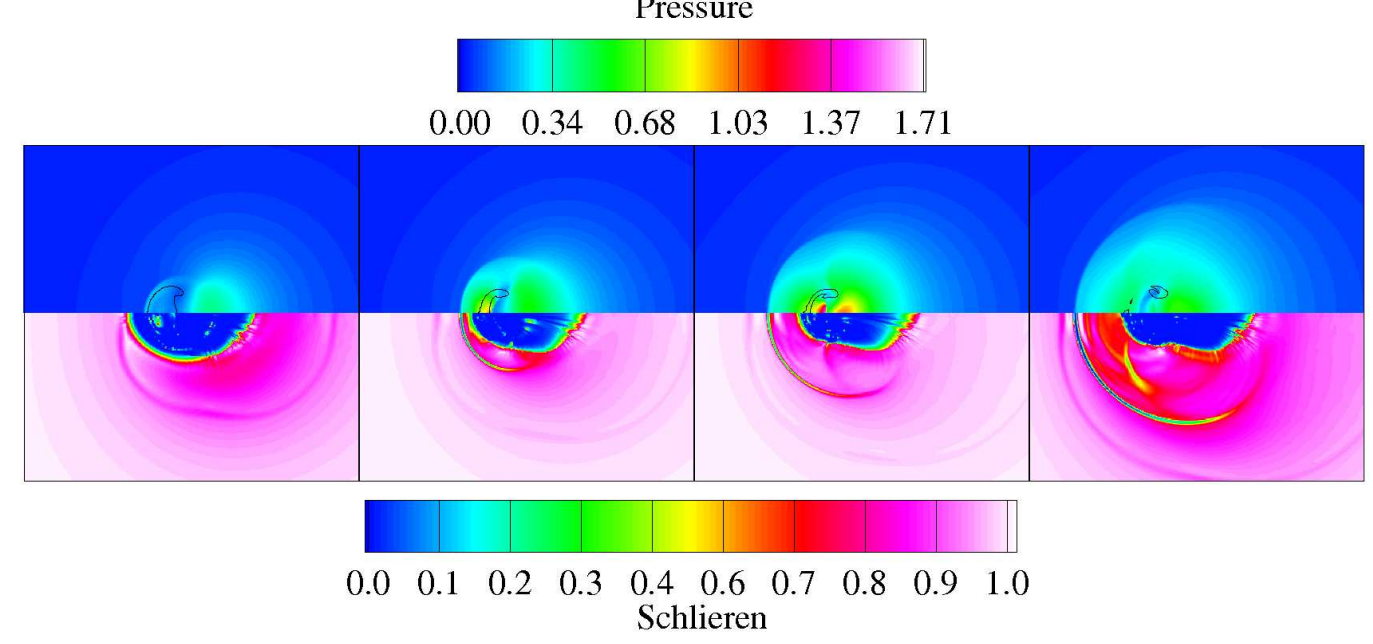

Figure 4.37: Flow field just after the impact of the jet onto the distal side for Rayleigh collapse near a wall $\left(p_{s} / p_{o}=714, H_{o} / R_{o}=1.5\right)$ at $t c_{L} / R_{o}=5.56,5.69$, 5.82, 5.95. Top: pressure, velocity, density, and $\gamma$ profiles along $r=0$; bottom: pressure and numerical Schlieren contours.

the bubble is highly compressed, so that it emits a gas compression shockwave simultaneously with the water-hammer pressure. 


\section{Free-field shock-induced collapse}

Free-field shock-induced collapse $\left(p_{s} / p_{o}=353\right)$ is considered to study the diffraction of the incoming shock, and the generation of the water-hammer shock.
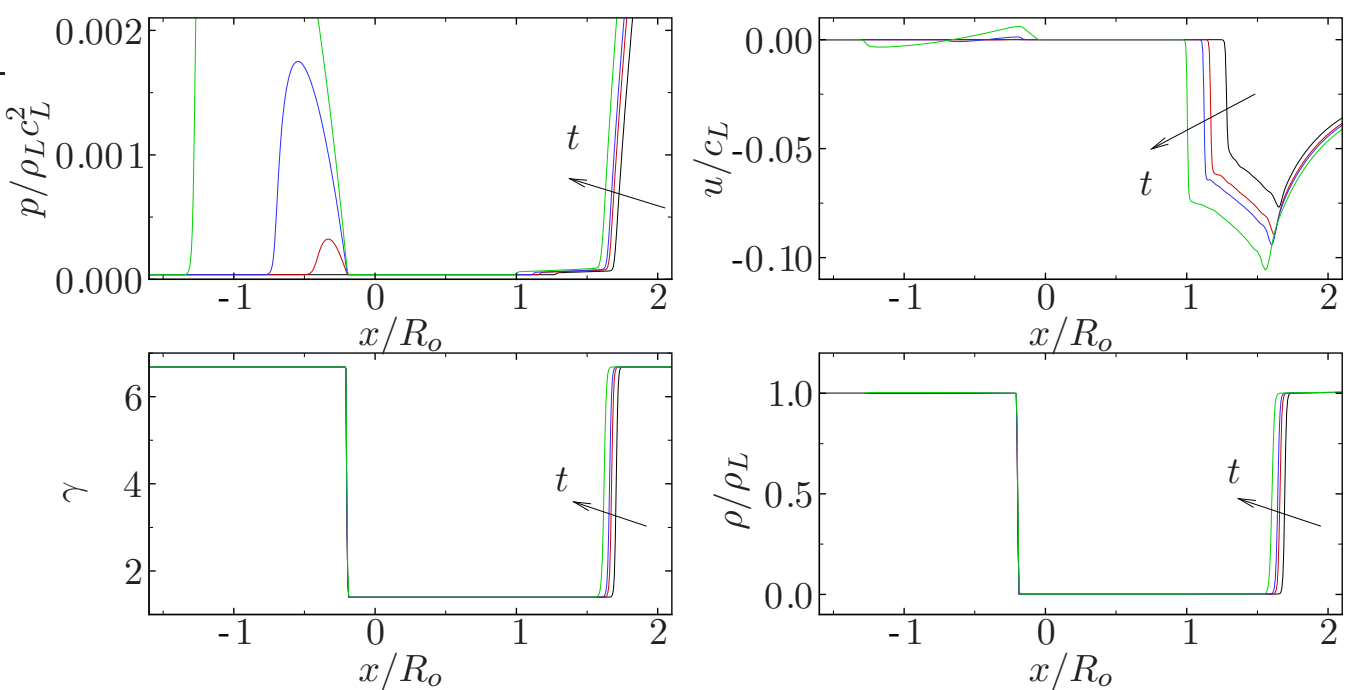

Pressure

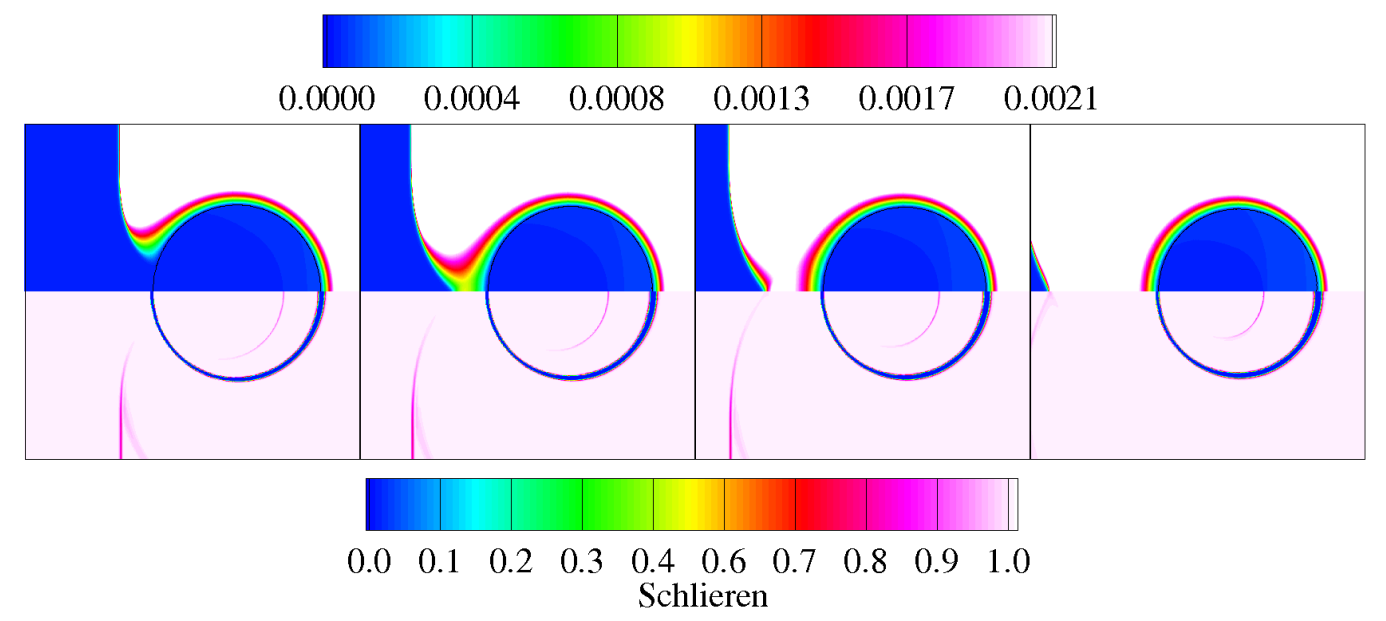

Figure 4.38: Flow field as the shock diffracts off the bubble for free-field shockinduced collapse $\left(p_{s} / p_{o}=353\right)$ at $t c_{L} / R_{o}=3.73,4.20,4.39,4.85$. Top: pressure, velocity, density, and $\gamma$ profiles along $r=0$; bottom: pressure and numerical Schlieren contours.

Figure 4.38 shows the diffraction of the incoming shock about the bubble at $t c_{L} / R_{o}=3.73,4.20,4.39,4.85$. Initially, a portion of the incoming shockwave is 
transmitted, while most of it is reflected. As the incoming shock propagates past the top of the bubble, it diffracts off its surface and intersects with itself along $r=0$. This phenomenon starts the collapse on the distal side. A small-amplitude compression wave propagates into the bubble from the distal side and interacts with the transmitted shock, which is being focused.
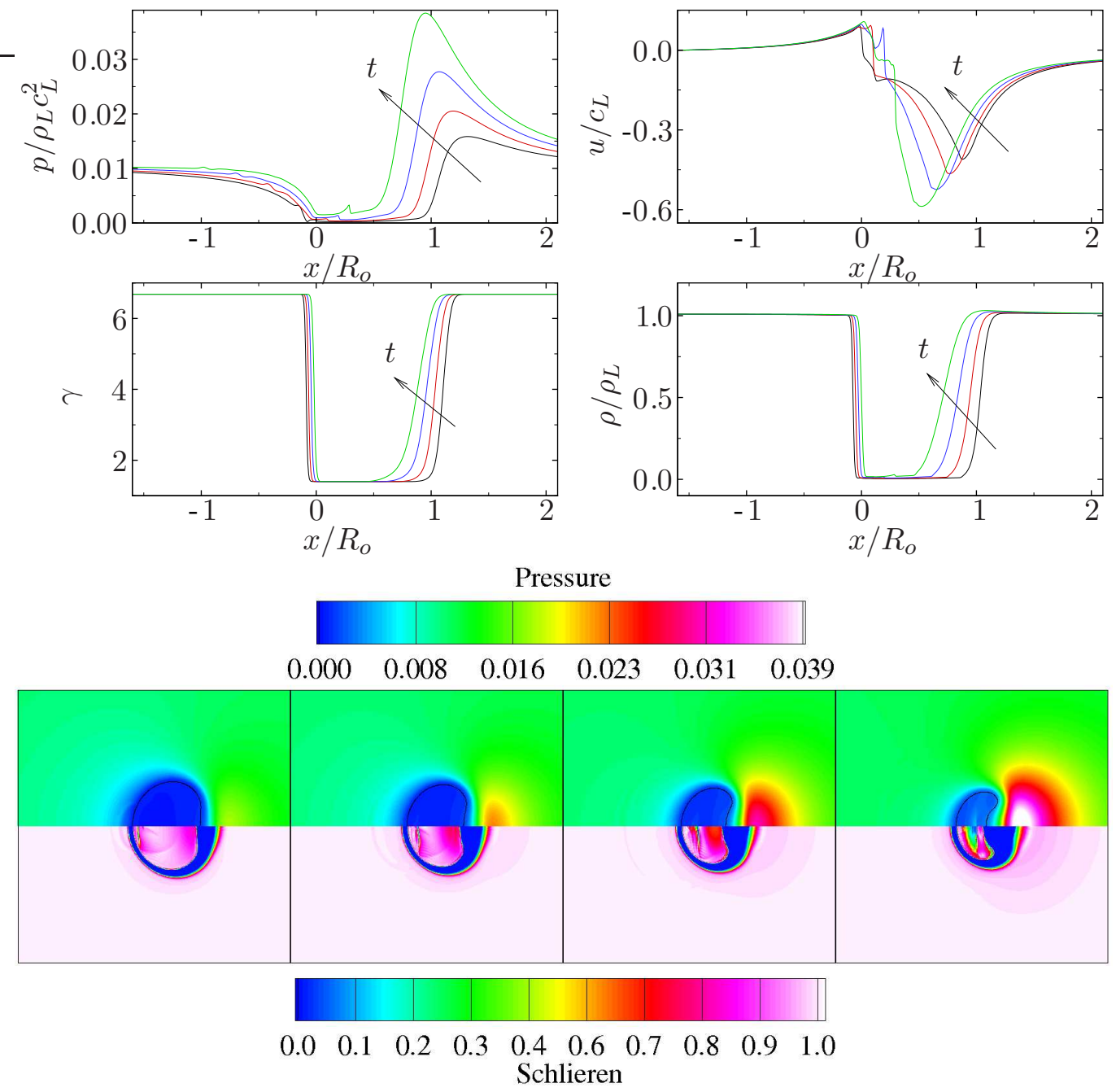

Figure 4.39: Flow field just before the jet hits the distal side for free-field shockinduced collapse $\left(p_{s} / p_{o}=353\right)$ at $t c_{L} / R_{o}=8.40,8.68,8.96,9.24$. Top: pressure, velocity, density, and $\gamma$ profiles along $r=0$; bottom: pressure and numerical Schlieren contours. 
Figure 4.39 shows the jet formation at $t c_{L} / R_{o}=8.40,8.68,8.96,9.24$. In the latter stages of collapse, the pressure behind the proximal side increases significantly due to the acceleration of the bubble interface. Similarly, the pressure at the distal side increases, but to a lesser extent, because the interface velocity has not yet reached the fast growth regime. The small kink observed within the bubble is the shock transmitted into the bubble at the first shock-interface interaction; the transmitted shock has reflected off the distal side and is traveling back towards the proximal side.

Figure 4.40 shows the generation of the water-hammer pressure wave at $t c_{L} / R_{o}=$ $9.43,9.61,9.80,9.99$. The gas within the bubble is highly compressed at this time and the shock dynamics within the bubble are more complex. The precursor shock, generated by the piston-like motion of the jet, is more difficult to identify than in the previous section, because the internally reflected shock interfers with it. It exits the bubble just before the water-hammer occurs. A sharp discontinuity develops in the pressure and velocity in the liquid on the proximal side due to the impact of the jet onto the distal side. The water-hammer shock generated by this process propagates through the bubble and is sharper in the direction of motion of the jet. The amplitude of the precursor shock is much smaller than that of the water-hammer shock.

Figure 4.41 shows the propagation of the waves after the water-hammer at $t c_{L} / R_{o}=10.2,10.5,10.7,11.2$. The maximum pressure (approximately $4.33 \mathrm{GPa}$ ) and jet velocity are achieved in Figure 4.41. The water-hammer shock propagates radially outwards. As shown in Section 4.5.4, the pressure decays as $1 / r$.

\subsubsection{Water-hammer pressure}

When a high-speed liquid impacts a solid, a water-hammer pressure is generated and obeys the equation (Huang et al., 1973)

$$
p_{w h}=\rho_{L} c_{L} v_{j} \frac{\rho_{S} c_{S}}{\rho_{S} c_{S}+\rho_{L} c_{L}}
$$



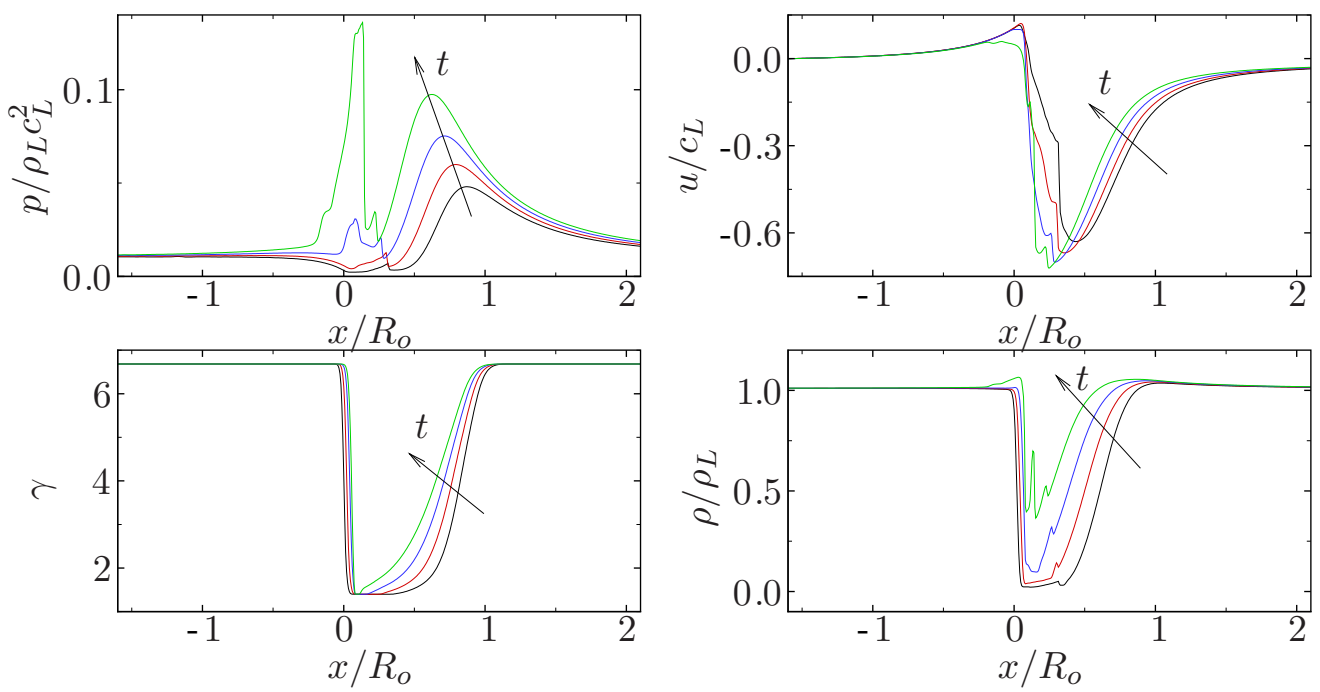

Pressure

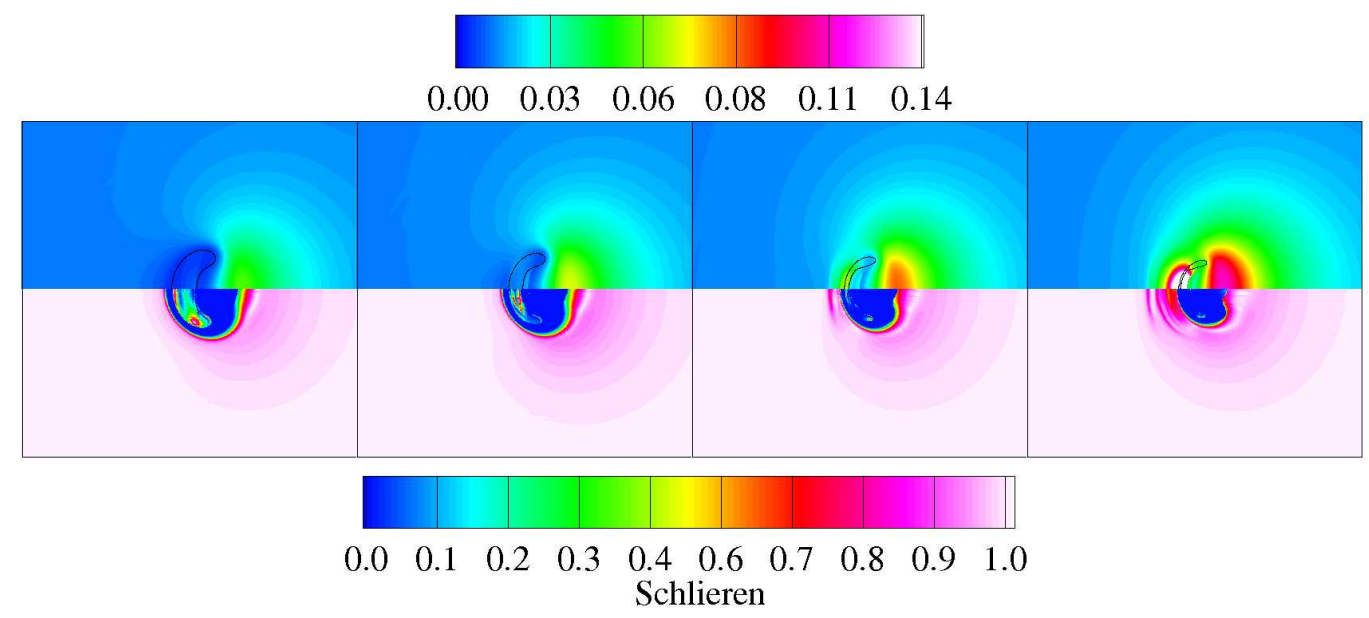

Figure 4.40: Flow field when the water-hammer is generated for free-field shockinduced collapse $\left(p_{s} / p_{o}=353\right)$ at $t c_{L} / R_{o}=9.43,9.61,9.80,9.99$. Top: pressure, velocity, density, and $\gamma$ profiles along $r=0$; bottom: pressure and numerical Schlieren contours.

where $\rho_{S}$ is the solid density and $c_{S}$ is the sound speed in the solid. In the present work, the re-entrant jet always hits the distal side first, which consists of water and moves at a velocity, $v_{d}$. The jet may hit the wall afterwards, but its velocity is greatly reduced by the impact. Thus, the water-hammer shock is always generated within the liquid in the problems of interest. For such a water/water impact, the 

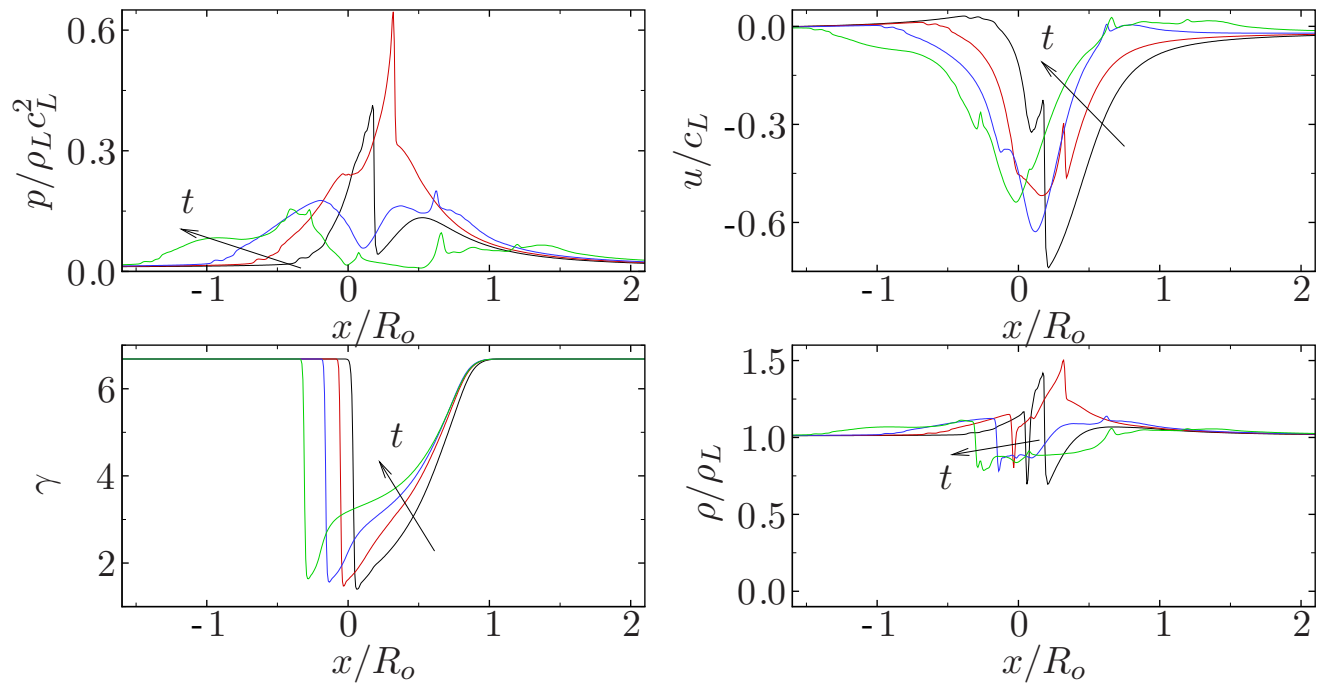

Pressure

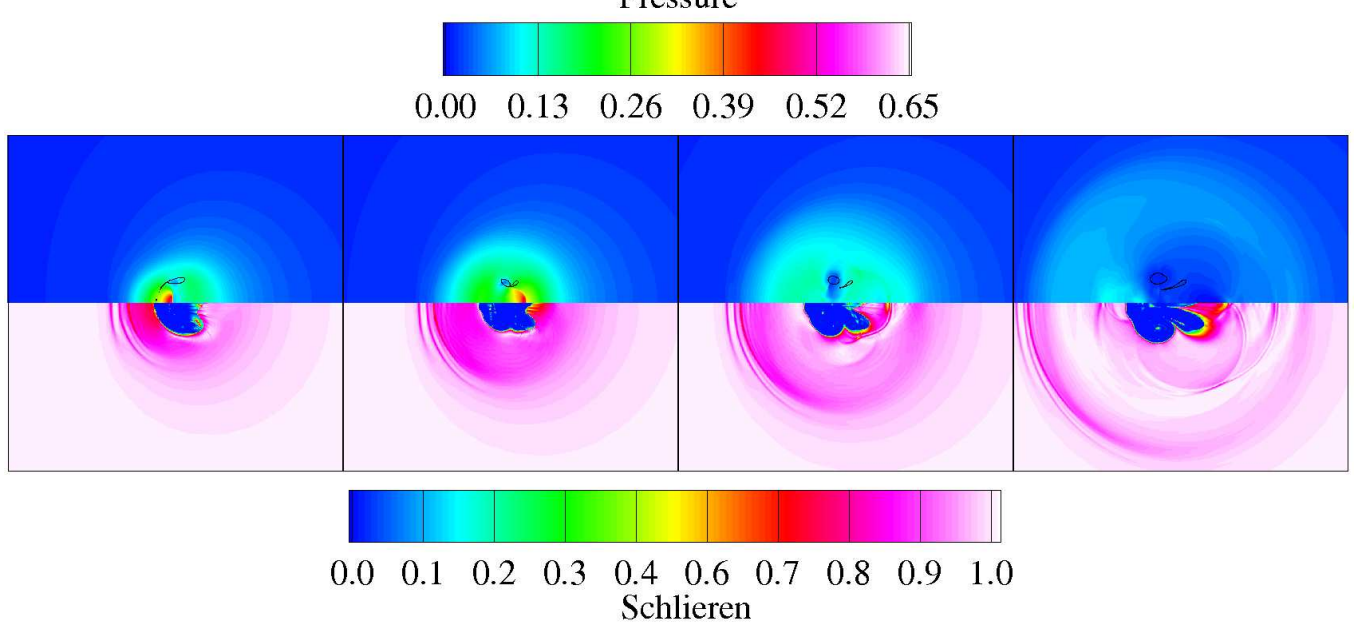

Figure 4.41: Flow field just after the jet hits the distal side for free-field shockinduced collapse $\left(p_{s} / p_{o}=353\right)$ at $t c_{L} / R_{o}=10.2,10.5,10.7,11.2$. Top: pressure, velocity, density, and $\gamma$ profiles along $r=0$; bottom: pressure and numerical Schlieren contours.

water-hammer equation reduces to

$$
p_{w h} \approx \frac{\rho_{L} c_{L} \Delta v}{2}
$$

where $\Delta v=\left|v_{j}-v_{d}\right|$. The properties of the jet are measured according to the procedure described in Appendix C. Figure 4.42 shows the water-hammer pressure 
computed based on Equation 4.36 and measured in the simulations for free-field shock-induced collapse, shock-induced collapse near a wall $\left(p_{s} / p_{o}=353\right)$ and Rayleigh collapse near a wall $\left(p_{s} / p_{o}=714\right)$. Results for free-field Rayleigh collapse are not included because no jet is generated in this case.
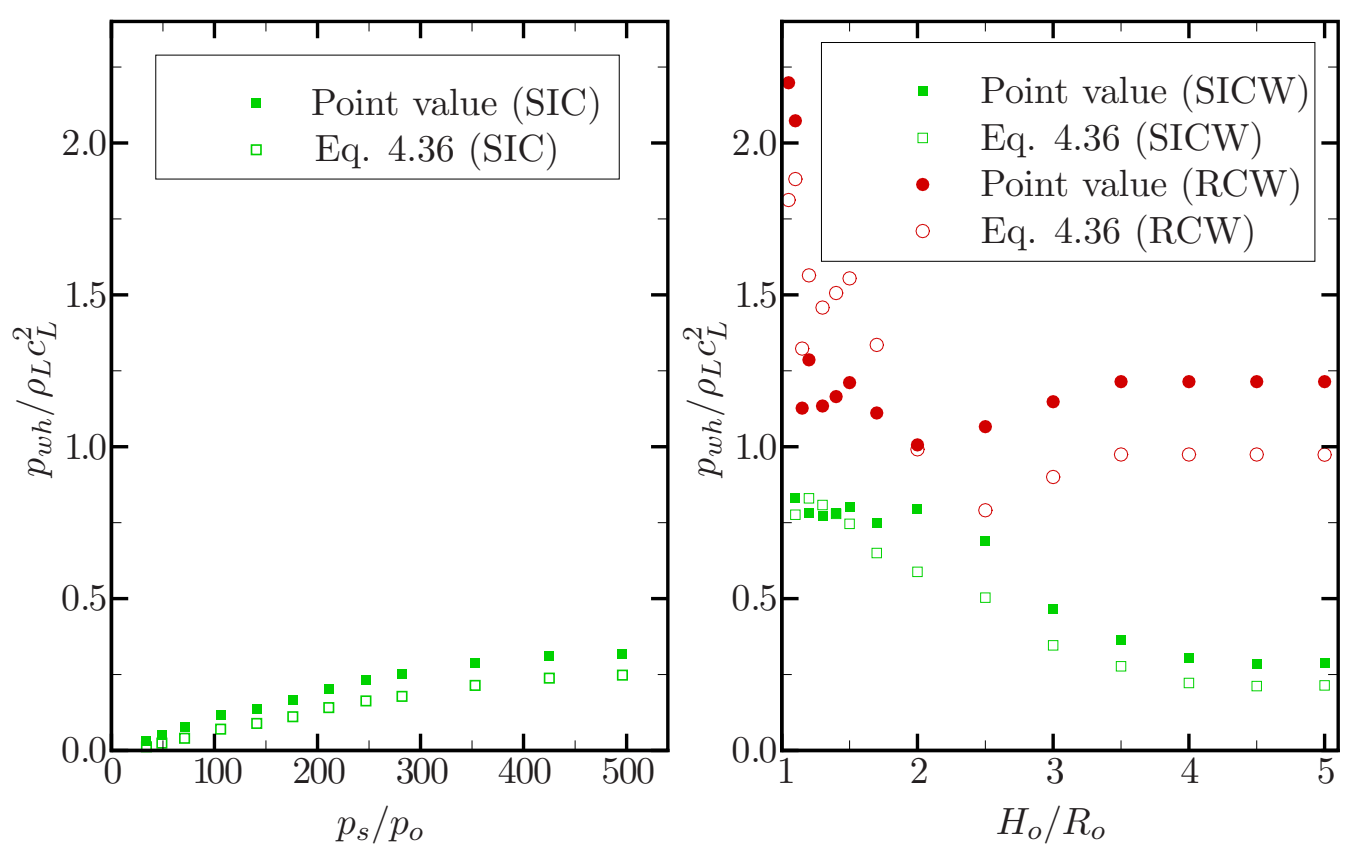

Figure 4.42: Water-hammer pressure as a function of the shock amplitude for freefield shock-induced collapse (left) and of the initial stand-off distance for shockinduced collapse $\left(p_{s} / p_{o}=353\right)$ and Rayleigh collapse $\left(p_{s} / p_{o}=714\right)$ near a wall.

As expected, the water-hammer pressure increases with increasing pressure ratio for free-field shock-induced collapse. The agreement between the theory and the simulation is good initially. However, the theory slightly underpredicts the measured water-hammer pressure. In the case of collapse near a wall, the maximum water-hammer pressure occurs at small $H_{o} / R_{o}$. The agreement between the values measured in the simulations with the theory is acceptable in shockinduced collapse, though the theory is underpredicted again. In Rayleigh collapse near a wall, the re-entrant jet forms and impact the distal side until $H_{o} / R_{o} \approx 2.5$; beyond this distance, the emitted shockwave is caused by the compression of the 
gas, because the jet does not penetrate the bubble sufficiently to impact the distal side and generate a water-hammer shock. The maximum value is achieved near the wall, where $p_{w h, \max } \approx 1.49 \mathrm{GPa}$, in agreement with Jamaluddin (2005).

For the present range of $H_{o} / R_{o}$, the jet always impacts the distal side first and subsequently decelerates. Hence, the water-hammer is always generated within the liquid. If the bubble is close enough to the wall, the jet may hit it directly at collapse. This is the case in the experiments of Tomita \& Shima (1986), Vogel et al. (1989) and Philipp \& Lauterborn (1998), where $H_{o} / R_{o}<1 .^{13}$ However, as shown by the history of the jet velocity in Section 4.4, the jet greatly decelerates after impacting the distal side. Thus, the distal side would have to be extremely close to the wall in order for the jet to hit the wall at a very high speed.

\subsubsection{Radiated energy}

The collapse of a cavitation bubble is accompanied by the emission of a shockwave, which is an exhibition of energy loss via liquid compressibility during the process. Vogel et al. (1989) report that the average energy loss of a cavitation bubble during the first collapse is $84 \%$, and that up to $90 \%$ of that fraction is due to the emission of sound. From Equation 4.37, the amount of acoustic radiation is given by:

$$
E_{\text {rad }}=4\left(\frac{4 \pi}{3} R^{3} \rho_{o}\right) \frac{\dot{R}^{2}}{2} \frac{\dot{R}}{c_{o}}+\int_{R_{o}}^{R} \frac{1}{c_{o}}\left(-3 \gamma p_{B} \frac{\dot{R}}{R}\right)\left(1-\frac{\dot{R}}{c_{o}}\right) 4 \pi R^{3} d R
$$

Because the collapse is not purely spherical, $R$ and $\dot{R}$ are computed as described in Section C.2.4. The total energy is given by

$$
E_{t o t}=\frac{4}{3} \pi R_{o}^{3}\left(p_{s}-p_{o}\right)
$$

Figure 4.43 shows the fraction of the normalized radiated energy, $E_{r a d} / E_{t o t}$, and the maximum average bubble pressure as a function of the initial pressure

\footnotetext{
${ }^{13}$ The initial shape of bubbles for which $H_{o} / R_{o}<1$ is not spherical. It is therefore not clear how to make comparisons between such bubbles and spherical bubbles.
} 
ratio for free-field Rayleigh collapse and shock-induced collapse, while Figure 4.44 shows the fraction of radiated energy and the maximum bubble pressure as a function of the initial stand-off distance for Rayleigh collapse with $p_{s} / p_{o}=714$ and shock-induced collapse with $p_{s} / p_{o}=353$ near a wall.
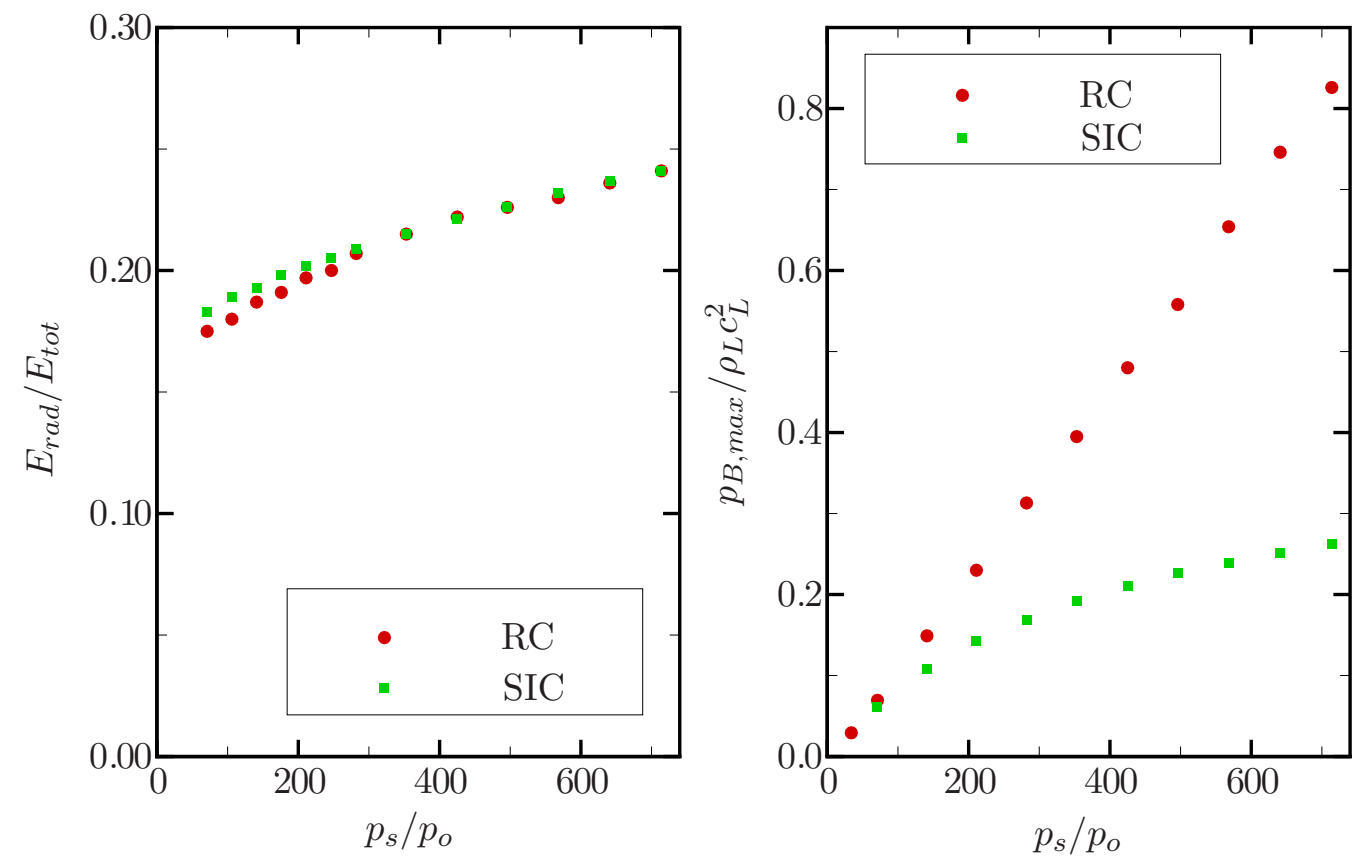

Figure 4.43: Fraction of radiated energy and maximum bubble pressure as a function of the pressure ratio for free-field shock-induced collapse.

In free-field collapse, even though the dynamics of collapse shown in Section 4.3 and the bubble pressure depend significantly on the pressure ratio, the energy radiated in shock-induced collapse is very close to that radiated during Rayleigh collapse, though it is a little bit higher in the case of low pressure ratios. This suggests that the details of the collapse are not very important, but that the pressure driving the collapse, $p_{s} / p_{o}$, is the parameter that governs the energy radiation, regardless of the type of collapse (shock-induced collapse or Rayleigh collapse).

In collapse near a wall, the energy radiated and the pressure increase with the initial stand-off distance, which is consistent with the experiments of Vogel et al. 

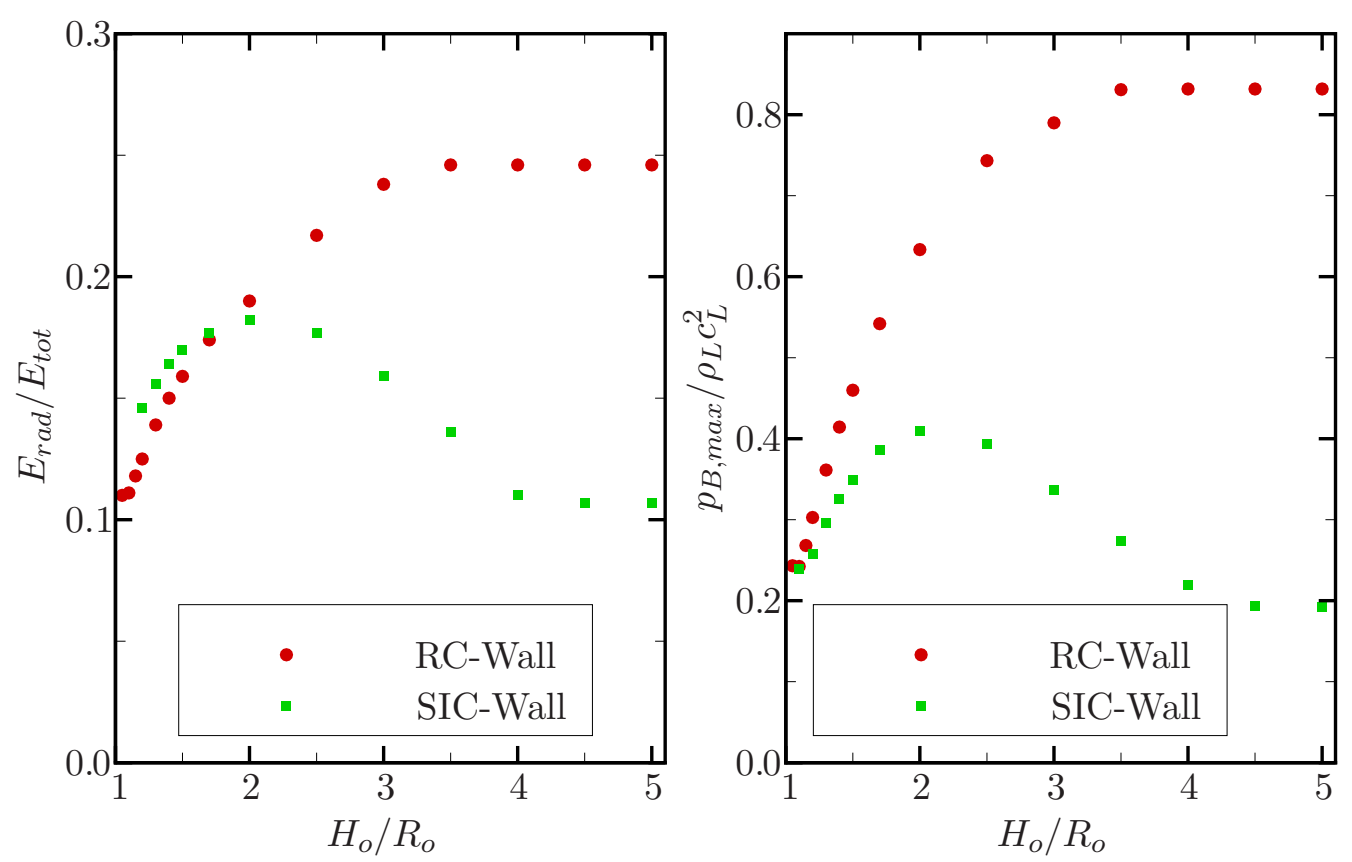

Figure 4.44: Fraction of radiated energy and maximum bubble pressure as a function of the initial stand-off distance for Rayleigh collapse near a wall.

(1989), though much lower. This trend may appear somewhat contradictory, in that energy is expected to go into the jet formation (Brennen, 2002). However, this can be related to the fact that the collapse becomes more spherical farther away from the wall, as shown in Section 4.3.4. For a given pressure ratio, a spherical collapse achieves a smaller radius and therefore a higher pressure, so that more energy is radiated (or lost). As a result, the rebound is smaller than for nonspherical collapse, where the bubble loses less energy and is therefore capable to rebound to a greater size. ${ }^{14}$

\footnotetext{
${ }^{14}$ This analysis of acoustic radiation during bubble collapse was motivated by experimental observations made by Dr. Michael Bailey and Wayne Kreider at the University of Washington, Seattle, who noticed that the rebound time of non-spherical bubbles is longer and the rebound size is larger than for spherical bubbles.
} 


\subsubsection{Wall pressure}

As a measure of the damage potential of bubble collapse, the pressure along the wall is recorded in shock-induced collapse and Rayleigh collapse.

\section{Shock-induced collapse near a wall}

Figure 4.45 shows the history of the wall pressure at different locations along the wall $\left(r / R_{o}=0,1,2,4\right)$, for two different initial stand-off distances in shock-induced collapse near a wall $\left(p_{s} / p_{o}=353, H_{o} / R_{o}=1.2,2.0\right)$.
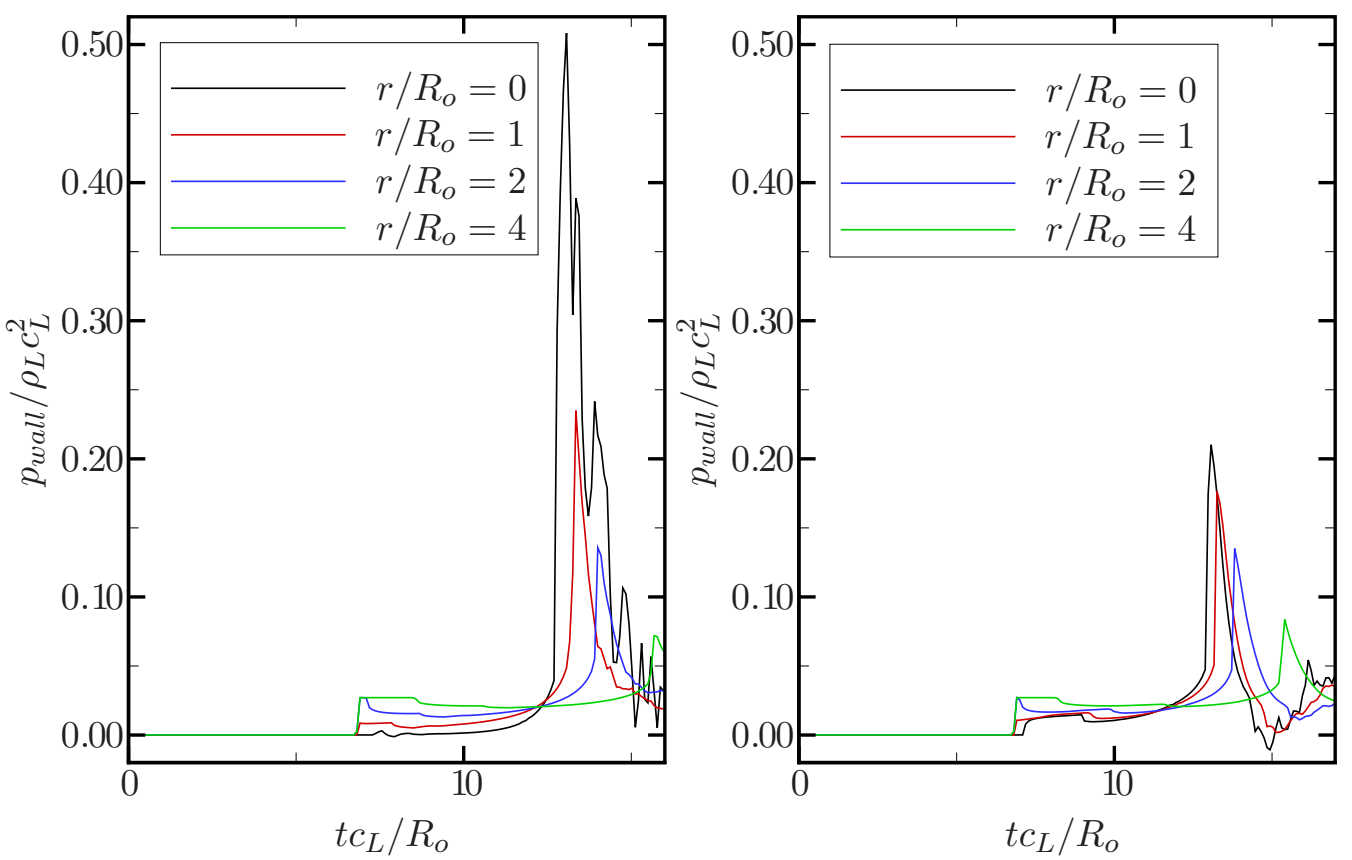

Figure 4.45: History of the wall pressure at different locations along the wall for shock-induced collapse near a wall $\left(p_{s} / p_{o}=353\right)$. Left: $H_{o} / R_{o}=1.2$; right: $H_{o} / R_{o}=2.0$.

The two situations show similar features. The pressure is initally atmospheric and suddenly increases when the shock reflects off the wall. As described in Section 4.5.6, the bubble interferes with the incoming shock in that a small portion is reflected, while the main shock diffracts about the bubble. Thus the shock in the shadow of the bubble is not as strong and is slightly delayed. After the shock re- 
flects off the bubble and inverts its phase, the wall pressure decreases. Then, a large and sharp pressure rise occurs due to the water-hammer shock. The subsequent increases and decreases are caused by later reflections between the water-hammer shock and the bubble. Significant tension may be achieved as expansion waves resulting from the shock reflection off the interface due to the impedance mismatch impact the wall, as shown in the plot for $H_{o} / R_{o}=2.0$. For small initial stand-off distances, the distal side barely moves, as shown in Section 4.3.5, and the bubble is very close to the wall. Thus, the wall pressure increases sharply along $r=0$ due to the water-hammer shock, but the measurements off-axis decrease rapidly. On the other hand, when the bubble is initially farther from the wall, the decrease in the wall pressure off-axis is smaller. This illustrates the fact that the water-hammer shock is a spherically propagating wave, so that, far from the wall, the wave appears nearly planar. Furthermore, as observed in the contour plots of the previous sections, the water-hammer shock is stronger in the direction of propagation of the jet; even though the shock propagates radially outwards, its strength is not uniform. Even in the case $H_{o} / R_{o}=1.2$, the impact of the jet onto the wall is negligible.

The maximum wall pressure is thus of interest when estimating the potential damage. Figure 4.46 shows the maximum wall pressure as a function of the location of the bubble at collapse ${ }^{15}$ for shock-induced collapse with $p_{s} / p_{o}=353$, at different positions along the wall $(r=0,1,2,4)$.

The pressure is very high along the centerline and decays as $1 / r$. Values up to 2.3 GPa are achieved locally; these are consistent with the results of Jamaluddin (2005). Off the axis, the pressure is lower for bubbles very close to the wall; this may be explained by the fact that for such bubbles the shock propagates almost parallel to the wall, so that there is little reflection. The fact that bubbles initially near the wall migrate towards it significantly leads to higher wall pressures (Tomita \& Shima, 1986), even though the collapse is more gentle near the wall. Based on

\footnotetext{
${ }^{15}$ During collapse, the bubble moves towards the wall, so that this displacement must be taken into account when computing the pressure dependence on $r$, as explained in Section 4.3.8.
} 


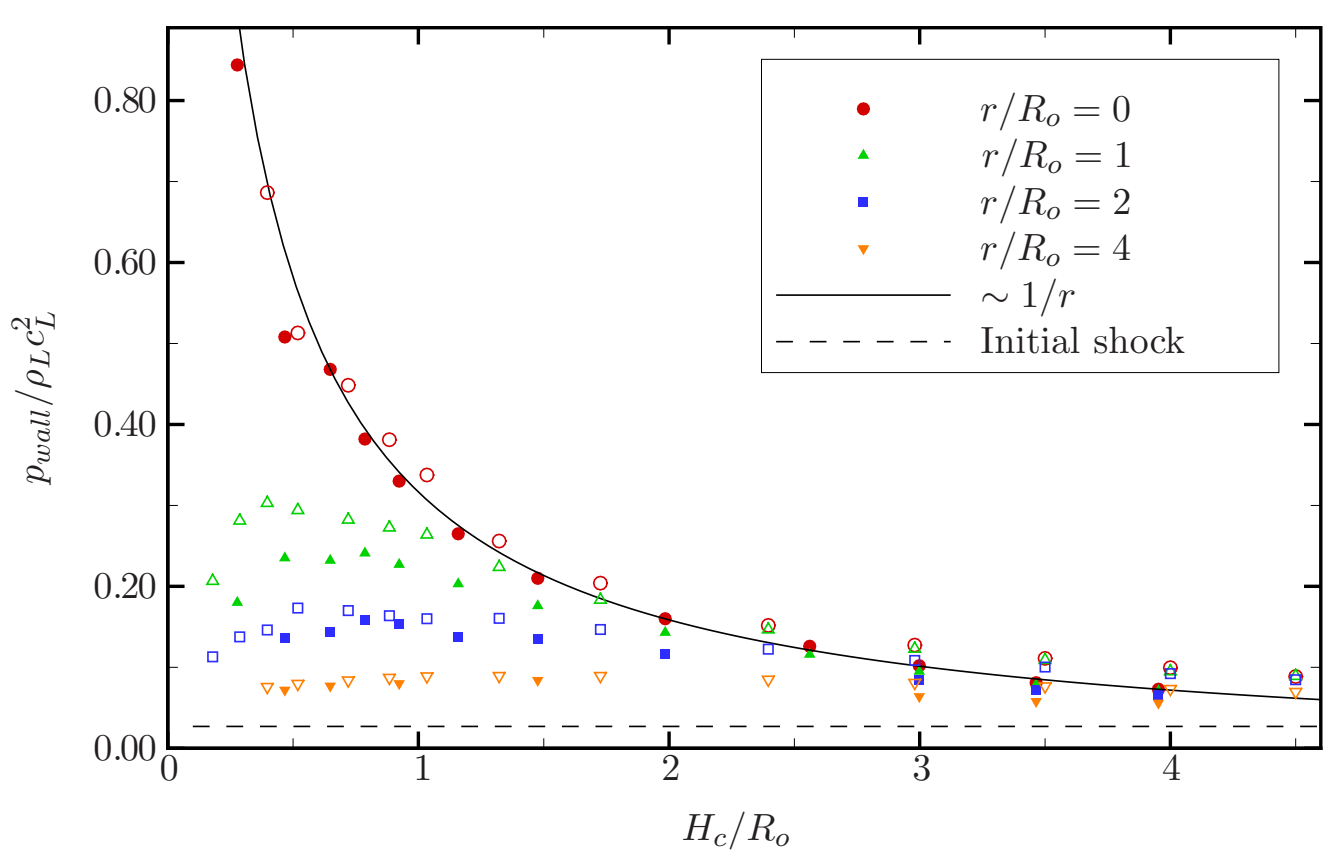

Figure 4.46: Maximum wall pressure as a function of the location of the bubble at collapse for shock-induced collapse $\left(p_{s} / p_{o}=353\right)$. The open symbols represent the equivalent Rayleigh collapse near a wall, with $p_{s} / p_{o}=714$.

the $1 / r$ relationship observed in Figure 4.46, a bubble needs to be located within $H_{\text {max }} \approx 7.9$ in order to generate a pressure as large as the incoming shockwave. In the experiments of Tomita et al. (1983) and Shima et al. (1984), a crossover value of approximately 7 is found for a $5 \mathrm{MPa}$ shock. As noted in Section 4.3.8, this corresponds to a pressure ratio of $p_{s} / p_{o}=25$ in the present simulations. Figure 4.47 provides a comparison of the present simulations with those experiments, where the wall pressure (averaged over the area of a hydrophone) and the wall pressure normalized by the incoming shock pressure are plotted as a function of the initial stand-off distance.

The same trend is observed in the experiments and in the simulations. Far from the wall, the pressure decreases with initial stand-off distance; near the wall, the pressure is not as high because it is averaged over a large area. However, the present simulations overpredict the wall pressure. In the experiments, the shock 

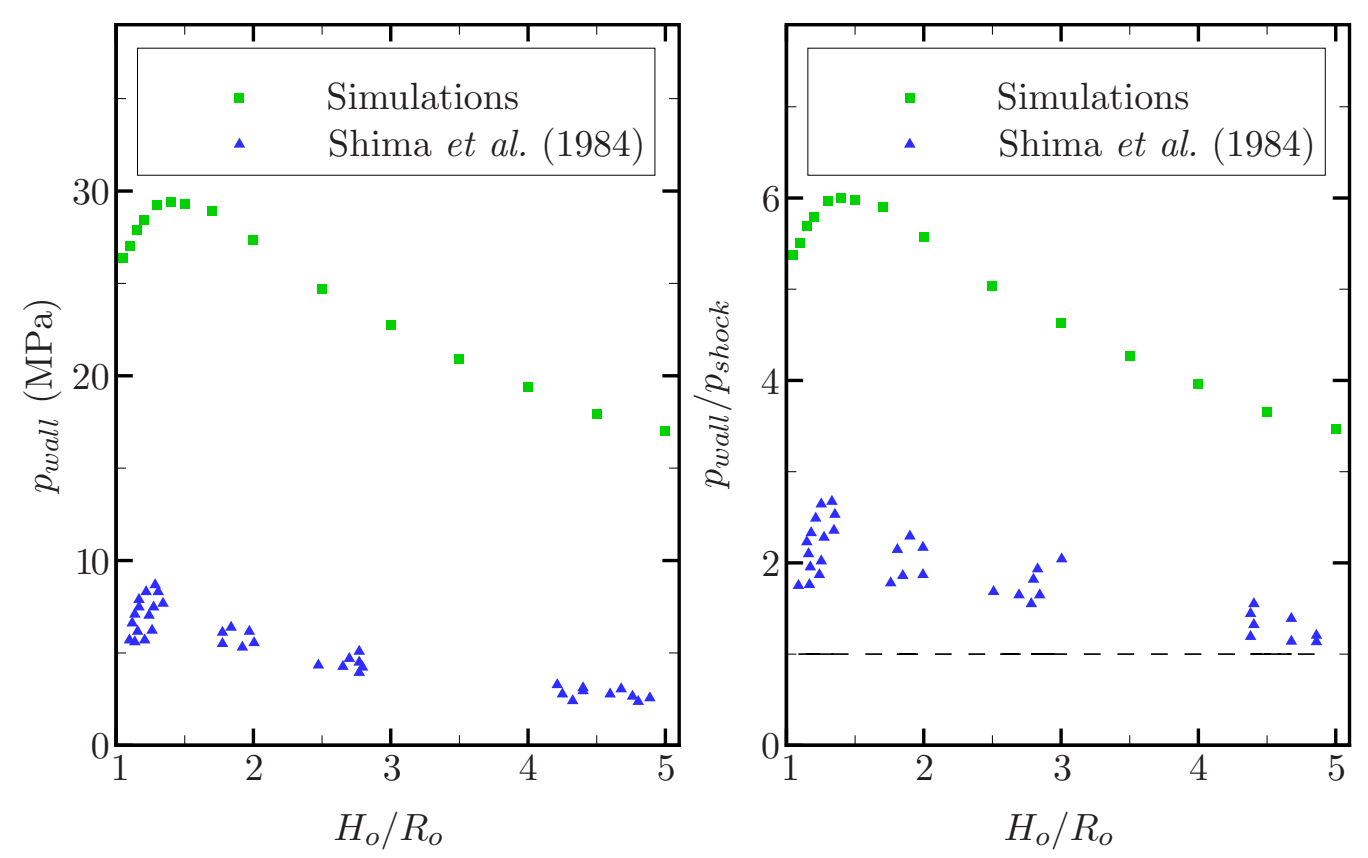

Figure 4.47: Maximum wall pressure as a function of the initial stand-off distance for shock-induced collapse $\left(p_{s} / p_{o}=25\right)$. Left: pressure in $\mathrm{MPa}$; right: pressure normalized by incident shock.

is generated by an imploding bubble, so that the amplitude of the shock decreases with distance; a calibration is performed to determine where the origin of the explosion must be located so that a $5 \mathrm{MPa}$ shock impacts the bubble. Thus, the effective pressure collapsing the bubble after the reflection of the shock off the wall is lower than $5 \mathrm{MPa}$ in the experiments. Because of the subtleties in representing the physics of the explosion process (Tanguay, 2004), the shock is planar with a constant amplitude in the present simulations, thus explaining the higher measured pressures. The effect of a finite pulse width is studied in Section 5.2.2.

In order to determine the extent of the potential damage, the area over which the pressure due to bubble collapse is larger than that of the incoming pulse is calculated. Figure 4.48 shows the wall pressure and its corresponding logarithm as a function of radial distance for $p_{s} / p_{o}=353$ and $H_{o} / R_{o}=2.0$. Several pressure profiles are shown, in addition to the maximum recorded pressure at any position. 

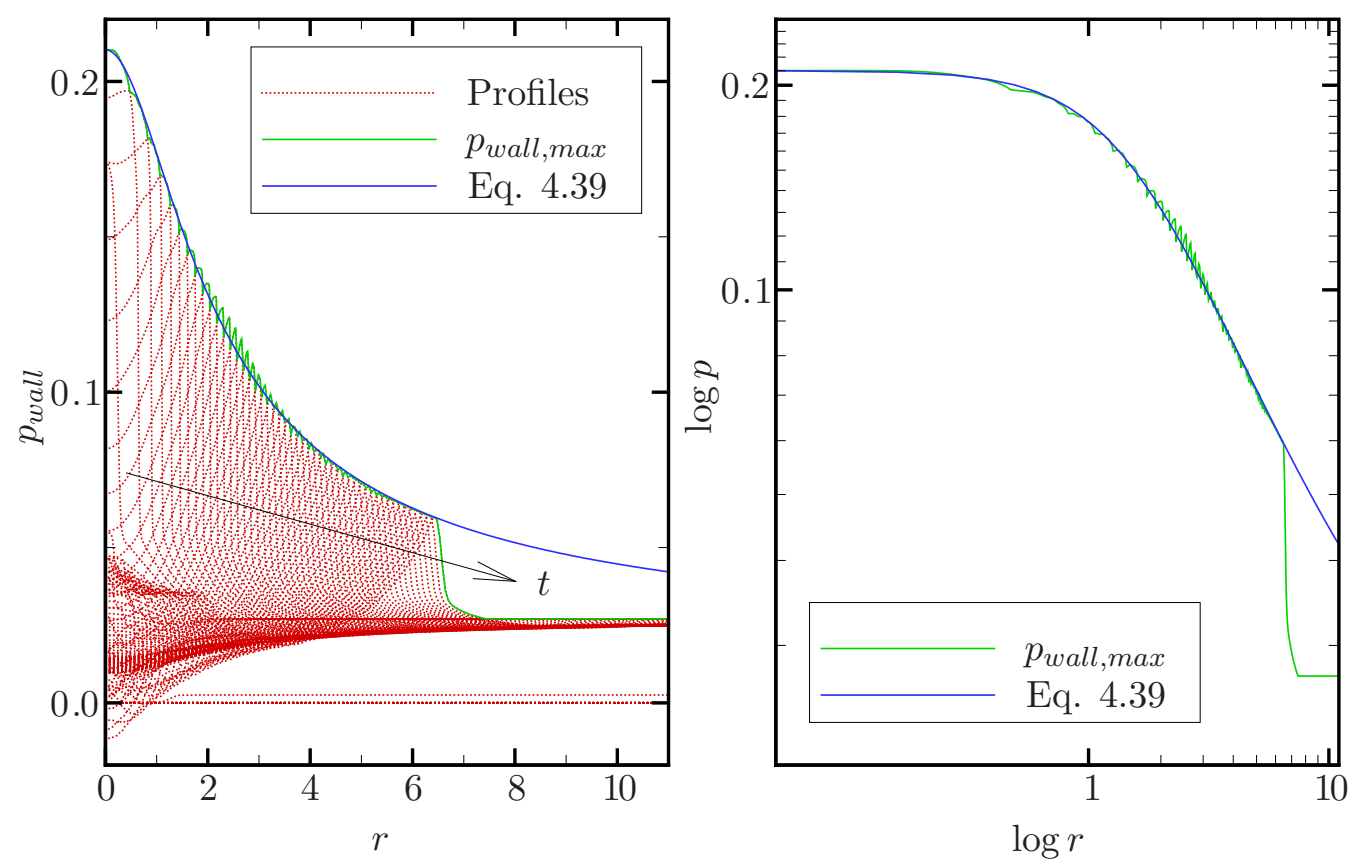

Figure 4.48: Wall pressure along the centerline for shock-induced collapse $\left(p_{s} / p_{o}=\right.$ $\left.353, H_{o} / R_{o}=2.0\right)$.

Because the water-hammer shock propagates spherically outwards, the pressure is inversely proportional to the radial distance from the origin of the shock (Hickling \& Plesset, 1964; Fujikawa \& Akamatsu, 1980), so that, along the wall,

$$
p_{w a l l}(r)=\frac{c_{1}}{\sqrt{H_{c}^{2}+r^{2}}}+c_{2}
$$

where $H_{c}$ is the distance from the collapse location to the wall for a particular $H_{o} / R_{o}$, and $c_{1}$ and $c_{2}$ are constants that can be determined if two pressure measurements are known. Equation 4.39 is the dashed curve in Figure 4.48, which matches the results very closely. This equation confirms the fact that the shock resulting from the collapse of a bubble far away from the wall (large $H_{c}$ ) looks essentially planar. Since the numerical simulation does not last long enough to show when the wall pressure due to the bubble collapse crosses over the shock pressure, the radius of the area over which the wall pressure is larger than that of 
the pulse is computed using Equation 4.39:

$$
L_{r}=\sqrt{\left(\frac{a}{p_{\text {pulse }}-b}\right)^{2}-H_{c}^{2}},
$$

where $p_{\text {pulse }}$ is the pressure of the incoming pulse. In this particular case, $L_{r} / R_{o} \approx$ 6.3 , meaning that the area over which the pressure due to bubble collapse is larger than that of the incoming shock is 40 times larger than the projected area of the bubble. Thus, although bubbles undergoing shock-induced collapse are initially small $\left(R_{o} \approx 10 \mu \mathrm{m}\right)$ and collapse to an even smaller size, the area over which they exert a pressure higher than that of the pulse is much larger $\left(R_{\text {area }} \approx 40 \mu \mathrm{m}\right)$.

\section{Rayleigh collapse near a wall}

Figure 4.49 shows the maximum wall pressure as a function of the location of the bubble at collapse for Rayleigh collapse with $p_{s} / p_{o}=34$, at different positions along the wall $\left(r / R_{o}=0,1,2,4\right)$.

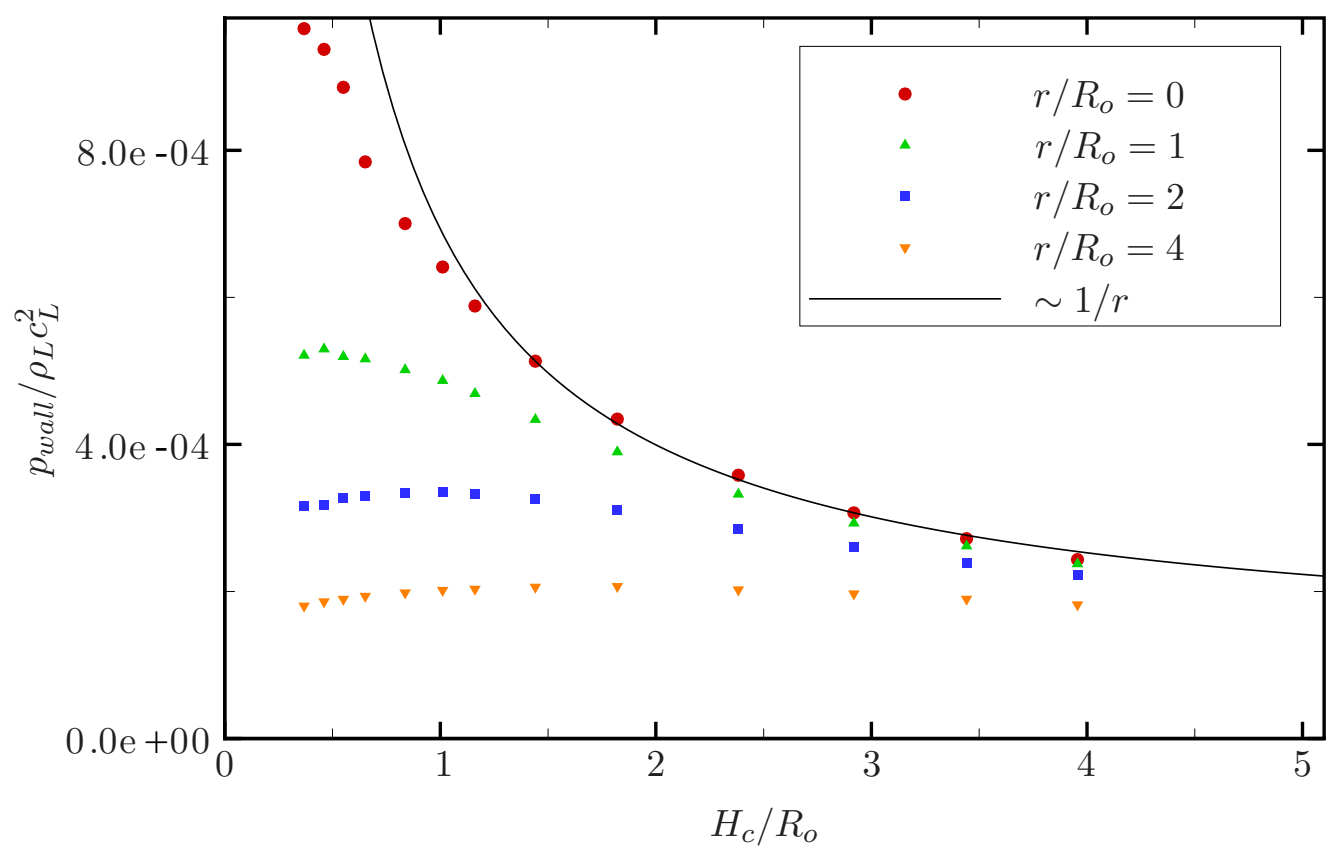

Figure 4.49: Maximum wall pressure as a function of the location of the bubble at collapse for Rayleigh collapse $\left(p_{s} / p_{o}=34\right)$. 
As observed in Hickling \& Plesset (1964) and Fujikawa \& Akamatsu (1980) for free-field Rayleigh collapse, the amplitude of the shockwave emitted upon collapse decays as $1 / r$. The departure from the $1 / r$ curve close to the wall is due to the fact that the collapse is less energetic; the presence of the wall leads to a slower collapse. Thus, even though bubbles near the wall migrate towards it by a greater amount than bubbles initially farther away, as described in Section 4.3.8, the more gentle collapse due to the presence of the wall, as described in Section 4.3.7, leads to a lower wall pressure. As illustrated previously, for a collapse very close to the wall, the pressure measured farther up along the wall (off-axis) is not as high as that of a bubble collapsing farther away from the wall.

Figure 4.50 shows the wall pressure as a function of the initial stand-off distance in order to compare to the experiments of Tomita \& Shima (1986). In the simulations, the pressure is averaged over an area equivalent to that of the hydrophone used in the experiments. The ratio of the radius of the hydrophone to that of the bubble is $R_{o} / R_{\text {hyd }}=0.63$.

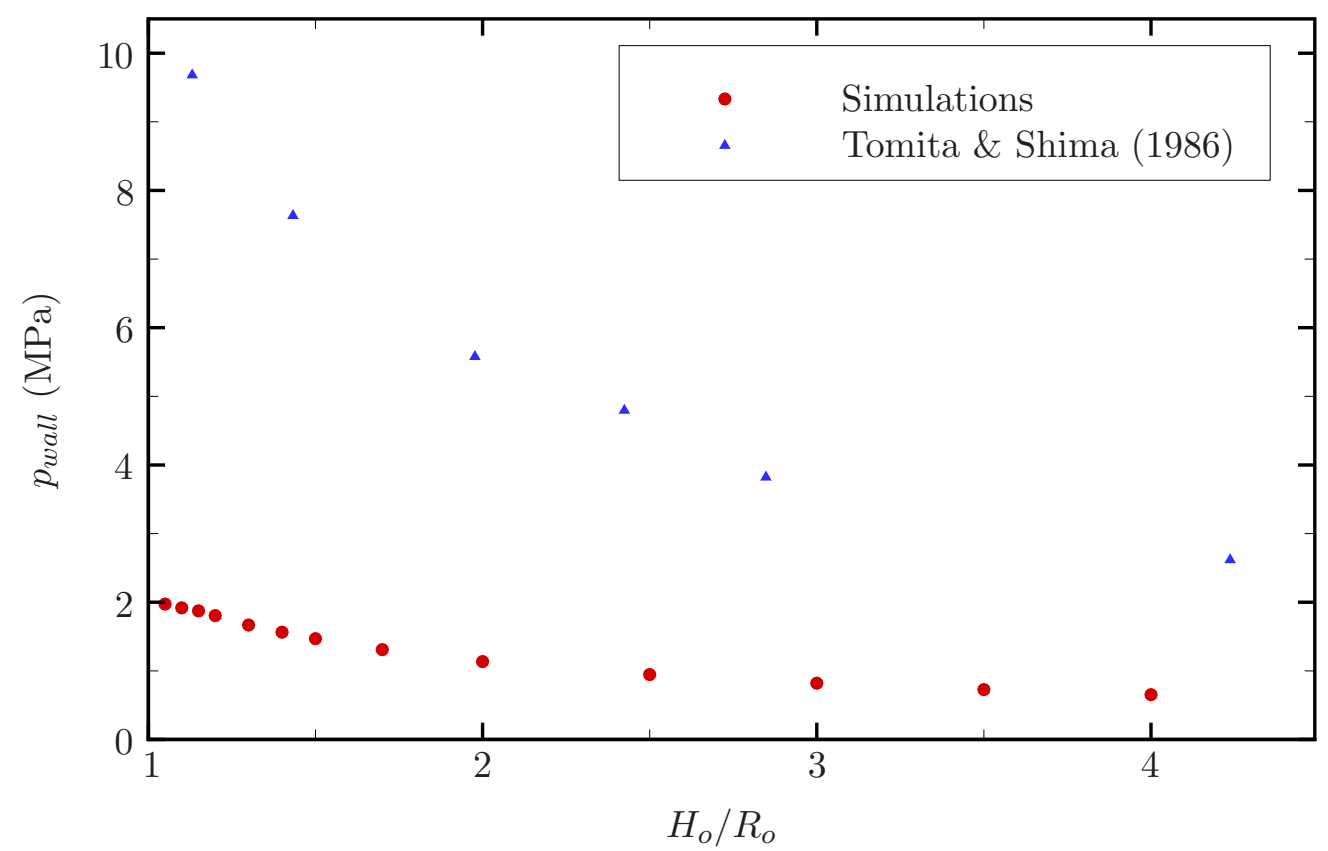

Figure 4.50: Averaged wall pressure as a function of the initial stand-off distance for Rayleigh collapse with $\left(p_{s} / p_{o}=34\right)$. 
Though the overall trend is captured, the results of the simulations show pressures lower than those obtained in the experiments. This is primarily due to the fact that the bubble is filled with non-condensible gas only in the simulations. From Rayleigh-Plesset analysis, the compression of the non-condensible gas arrests the bubble collapse and causes rebound. Thus, the minimum radius at collapse is larger in the simulations than it would be for a vapor bubble with the same initial radius, which contains far less non-condensible gas. For a bubble with little gas content undergoing spherical Rayleigh collapse, the asymptotic velocity at collapse is described by Equation 4.10. Thus, the minimum radius and bubble pressure can be expressed as

$$
\frac{R_{\min }}{R_{o}}=\left[\frac{p_{G o}}{(\gamma-1)\left(p_{\infty}-p_{v}\right)}\right]^{1 / 3(\gamma-1)}, \quad \frac{p\left(R_{\min }\right)}{p_{G o}}=\left[\frac{p_{G o}}{(\gamma-1)\left(p_{\infty}-p_{v}\right)}\right]^{\gamma /(\gamma-1)} .
$$

As an example, the equilibrium radius of a bubble is approximately $10 \mu \mathrm{m}$ and the maximum radius is approximately $1 \mathrm{~mm}$ in SWL. If the bubble only consists of gas at equilibrium, the partial pressure of the gas at maximum radius is then approximately $10^{-6} p_{a t m}$, at which point the bubble mainly consists of vapor. This leads to a minimum radius of $R_{\min } / R_{o} \approx 2 \times 10^{-5}$. In the present simulations, the bubble consists of gas at ambient pressure initially (i.e., at $p_{G o}=p_{a t m}$ ), which then leads to a larger minimum radius $\left(R_{\min } / R_{o} \approx 0.1\right)$ and therefore to a much lower bubble pressure.

\subsubsection{Dependence on the shock amplitude}

The dependence of the wall pressure on the shock amplitude and initial stand-off distance can be useful when considering shock propagation through a bubble cloud near a solid surface. Figure 4.51 shows the dependence of the wall pressure on the shock Mach number for shock-induced collapse with $H_{o} / R_{o}=2.0$.

As expected, the wall pressure increases with increasing shock Mach number 


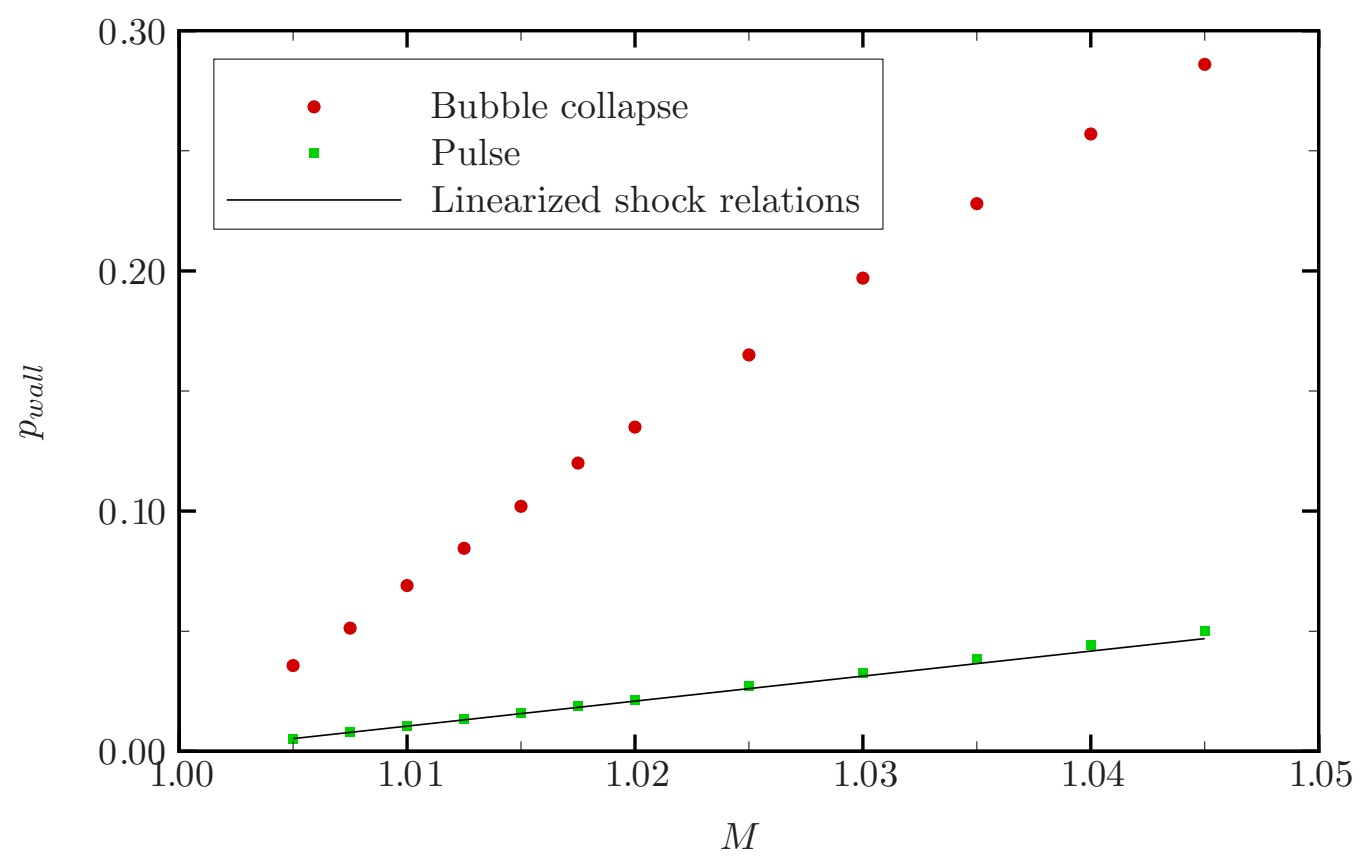

Figure 4.51: Maximum wall pressure as a function of the amplitude of the shockwave for shock-induced collapse near a wall $\left(H_{o} / R_{o}=2.0\right)$.

and matches the linearized shock relations, which are derived from Equation A.27:

$$
p_{\text {wall }, \text { shock }}=2\left(p_{o}+\frac{4}{\gamma+1} \epsilon\right)
$$

where the factor of two accounts for pressure doubling and $M=1+\epsilon$, with $0<\epsilon \ll 1$.

The damage due to the bubbles closest do the surface can be assessed by combining the present analysis with a model for shock propagation through bubbly mixtures (Kameda \& Matsumoto, 1996; Kameda et al., 1998). As the shock propagates through the cloud, it becomes attenuated, such that the bubbles nearest to the wall, which are likely to cause the most damage based on the analysis of Section 4.5.4, only feel a fraction of the original pressure ratio. Thus, if the initial stand-off distance and the shock amplitude are known, the results of this section can be used to predict the potential damage due to the shock-induced collapse of a single bubble. This can then be averaged over a given area to represent the 
damage due to multiple bubbles. It should be noted that each collapsing bubble emits a shockwave upon collapse, so that a given bubble is also subjected to such shockwaves.

\subsubsection{Shielding}

It has been shown in the previous sections that the effect of a bubble - in particular its collapse - can greatly amplify the pressure in shock-induced collapse near a wall. However, its mere presence causes part of the incoming shock to be reflected, so that the full extent of the shock is not felt along the wall. In other words, the bubble shields the wall. Figure 4.52 illustrates this effect by showing the pressure of the incoming shock along the wall at $r / R_{o}=0,1,2,4$ as a function of the initial bubble stand-off distance for shock-induced collapse with $p_{s} / p_{o}=353$.

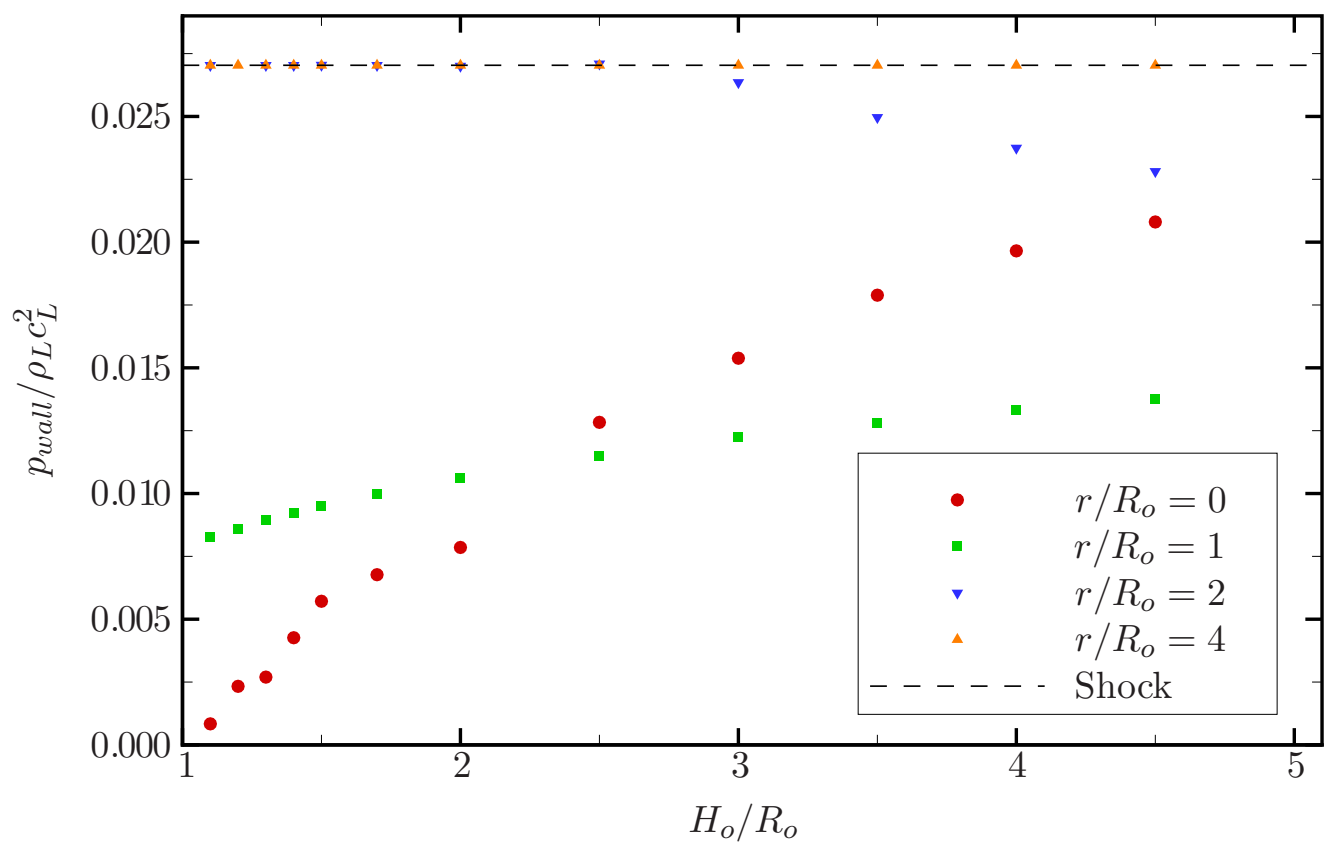

Figure 4.52: Maximum wall pressure due to the shock as a function of the initial bubble stand-off distance for shock-induced collapse near a wall $\left(p_{s} / p_{o}=353\right)$.

As expected, far from the axis $\left(r / R_{o}=4\right)$, the effect of the bubble is not felt and the wall pressure is that due to the shock. Conversely, at $r / R_{o}=0$, the 
pressure is greatly influenced by the presence of the bubble, which has reflected a portion of the incoming shock. For $r / R_{o}=2$ (i.e., outside of the shadow region), the presence of the bubble affects the pressure at large $H_{o} / R_{o}$. This is due to the fact that the shockwave diffracts around the bubble; this effect propagates from the curved part of the shock all the way to main front. Close to the axis, the pressure change due to the incoming shock is minimal for bubbles close to the wall. However, as the bubble is farther, the diffracted wave has time to intersect with itself and in fact strengthens as it propagates downstream. This phenomenon explains why the pressure is greater along the axis than at $r / R_{o}=1$, where the shock is curved, if the wall is far enough away. For bubbles very far from the wall, it is expected that the shockwave has had time to steepen again in the region near the axis to achieve a normal front.

\subsection{Summary of the bubble collapse results}

Results from numerical simulations of bubble collapse have been presented in this section. Overall, the simulations showed good agreement with the theory and experiments. For certain quantities, the results for Rayleigh collapse under cavitation conditions did not match the experimental results very well, because mass transfer is not included in the physical model.

The flow visualizations indicate that the following scenario occurs in bubble collapse near a wall, for the range of stand-off distances, $H_{o} / R_{o}=1.05-5$. A reentrant jet forms during collapse. When the jet impacts the distal side, it generates a large water-hammer pressure wave that propagates in the liquid and impacts the nearby solid surface. A gas compression shock is generated almost simultaneously and merges with the water-hammer pressure front. The strength of the shock is highest in the direction of propagation of the jet. Thus the damage mechanism in this case can be considered as a combination of two previously proposed damage mechanisms: the emission of the gas compression shock (Rayleigh, 1917) and the direct impact of the jet onto the solid surface (Kornfeld \& Suvorov, 1944). 
The dynamics of the collapse were characterized by considering a number of properties of the bubble and of the jet. The initial motion of the bubble and the collapse time match analytical results well. Jet velocities on the order to thousands of meters per second are achieved. The finite speed of propagation of shock affects certain properties of the collapse. If the bubble is located far from the wall, the reflected shock interacts with it late during the collapse, leading to a less energetic process.

The jet formation in shock-induced collapse was investigated qualitatively. Several mechanism are shown to play a role: the growth of non-spherical perturbations, baroclinic vorticity and the transient effects of shock propagation. In Rayleigh collapse near a wall, the presence of the wall leads to lower pressures driving the collapse.

The sequence of shockwave emission was determined. The previously observed gas compression and water-hammer shockwaves were visualized, and a precursor shock, generated by the piston-like motion of the jet, was observed. As a measure of damage due to single-bubble collapse, the pressure was computed along a nearby solid surface. Wall pressures on the order GPa were measured. The pressure decayed as $1 / r$, and it was determined that a bubble within a distance of $8 R_{o}$ generates as large a pressure as the incoming shock. For bubbles very close to the wall initially, the region over which the pressure due to bubble collapse is larger than that of the shock extends to $6 R_{o}$ along the wall. For small initial stand-off distances, the bubble migrates significantly towards the wall; however, the collapse is gentler because of the presence of the wall, so that the wall pressure is in fact slightly lower than expected by the $1 / r$ relationship. 


\section{Chapter 5}

\section{Single-bubble collapse in shockwave lithotripsy}

The results obtained in the previous chapter are now set into the context of shockwave lithotripsy (SWL). ${ }^{1}$ First, the effect of the finite width of the lithotripter pulse is discussed. Then, shock-induced collapse and Rayleigh collapse in the context of shockwave lithotripsy are considered. Finally, a mechanism of comminution for medium-sized stones and fragments is presented.

\subsection{Modeling of the lithotripter pulse}

The focal region of a lithotripter is characterized by a cigar-shaped region where the wavefront is nearly planar. For simplicity, the stone is assumed larger than this focal zone, which is much larger than a pre-existing spherical bubble. The lithotripter pulse is modelled as a compressive shock front of constant amplitude followed by a long expansion tail that typically includes a negative (tensile) pressure. The experimental waveform measured at the focus of an electro-hydraulic lithotripter is shown in Figure 1.4; other types of lithotripters generate at least qualitatively similar waveforms. The waveform can be represented by the following

\footnotetext{
${ }^{1}$ Results presented in this section have been submitted to the Journal of the Acoustical Society of America. Some sections may be redundant to some of the results in Chapter 4.
} 
analytical function (Church, 1989):

$$
p(t)=1+2 p_{s} e^{-\alpha t} \cos \left(\omega t+\frac{\pi}{3}\right)
$$

where $\alpha=4.5$ and $\omega=3.665$ in non-dimensional units; the parameters are chosen to closely match the waveform in a Dornier HM3 lithotripter. The pulse amplitude, $p_{s}$, and characteristic time, $T$, are defined in Figure 5.1; in particular, $T$ is equal to the second zero of the function given by Equation 5.1 (i.e., the time when the pressure becomes positive again after the first tensile region). The pulse width is then given by $\sigma=M_{s} T$, where $M_{s}$ is the shock Mach number. In the present simulation, the values of $\alpha$ and $\omega$ lead to a nominal value of $\sigma=6.75 \mathrm{~mm}$. Typical peak positive pressures at the focus range from 9-114 MPa and negative (tensile) pressures as low as -10 MPa have been measured (Coleman \& Saunders, 1989). In the present work, the amplitude of the compressive front is approximately 35 $\mathrm{MPa}$ with a very short rise time, while the tensile component is $-10 \mathrm{MPa}$. The positive pressure lasts for approximately $1 \mu \mathrm{s}$, while the negative pressure lasts for $4 \mu \mathrm{s}$.

In practice (Zhong et al., 1993; Cleveland et al., 2000), kidney stones have a finite impedance, approximately three to five times that of water. However, in the present work, the kidney stone is assumed to have infinite impedance, so that all waves are completely reflected, with no losses; thus, upon reflection of the pulse off the stone, the pressure doubles. This can be understood by considering the reflection of a planar shockwave in water off a solid surface, as illustrated in Figure 5.2. As noted in Thompson (1984), the change in velocity across the reflected shock has the same magnitude as the change in velocity across the incident shock and $u_{1}=u_{3}=0$. Using this information and Equations A.17a and A.17b from Appendix A,

$$
[p]_{r}\left[\frac{1}{\rho}\right]_{r}=[p]_{r}\left[\frac{1}{\rho}\right]_{i} .
$$

Combining this equation with Equation A.22, the following expression can be 


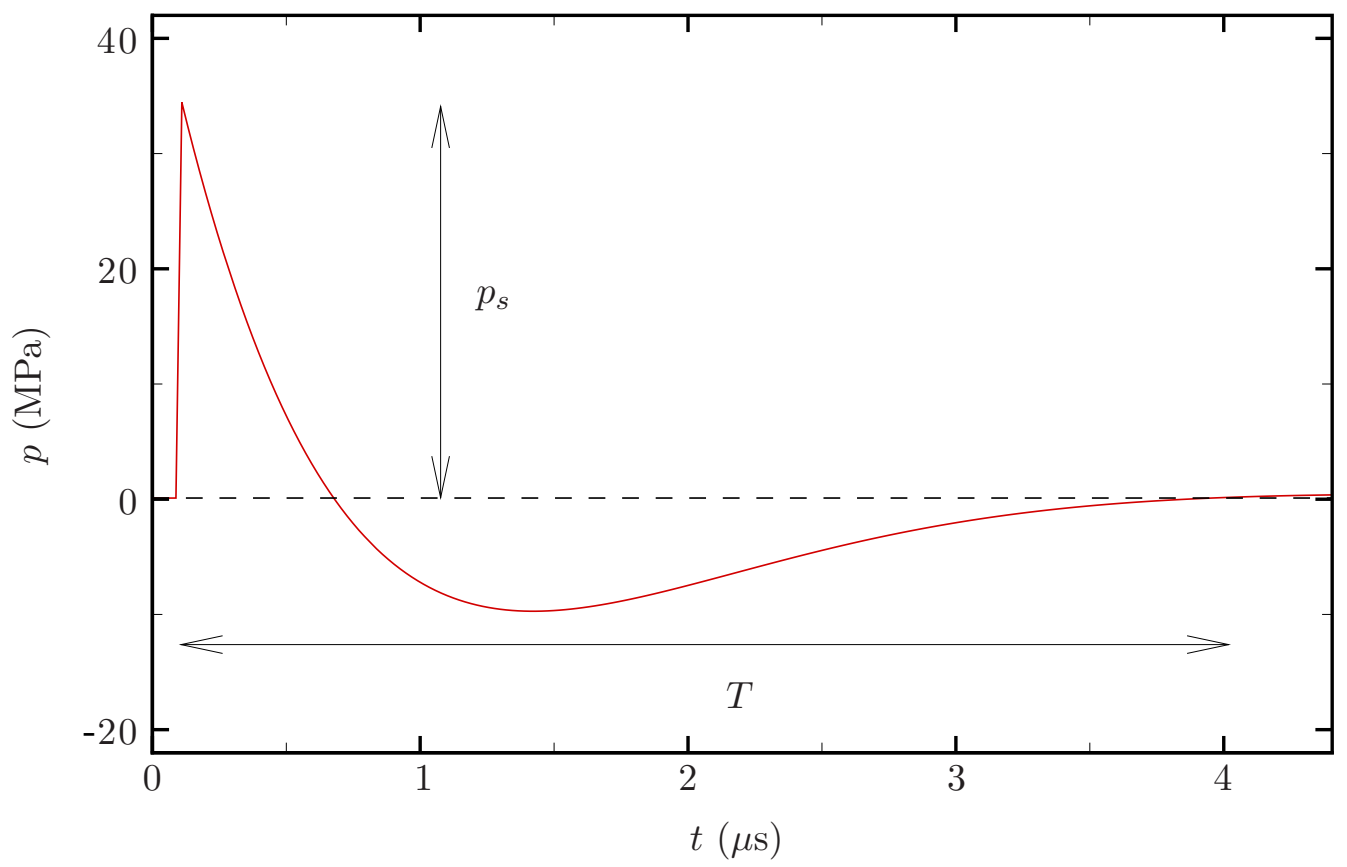

Figure 5.1: Pressure waveform of a lithotripter pulse at the focus.

derived:

$$
\left(p_{3}-p_{2}\right)\left(\frac{\frac{\gamma+1}{\gamma-1}+\frac{p_{3}+P_{\infty}}{p_{2}+P_{\infty}}}{\frac{\gamma+1}{\gamma-1} \frac{p_{3}+P_{\infty}}{p_{2}+P_{\infty}}+1}-1\right)=\left(p_{2}-p_{1}\right)\left(\frac{\frac{\gamma+1}{\gamma-1}+\frac{p_{2}+P_{\infty}}{p_{1}+P_{\infty}}}{\frac{\gamma+1}{\gamma-1} \frac{p_{2}+P_{\infty}}{p_{1}+P_{\infty}}+1}-1\right)
$$

In the case of gases, $P_{\infty}=0$, so that the relationship between $p_{3} / p_{2}$ and $p_{2} / p_{1}$ is usually nonlinear, unless the shocks are very weak and the acoustic limit is achieved. In the context of SWL, $P_{\infty} \gg p_{2}, p_{3} \gg p_{1}$, so that

$$
p_{3}=2 p_{2}-p_{1} \approx 2 p_{2}
$$

Thus, the pressure at the wall doubles upon reflection.

\subsection{Shock-induced collapse in SWL}

As described in Chapter 4, the potential for damage of shock-induced collapse is tremendous. However, it has not yet been considered for SWL. 


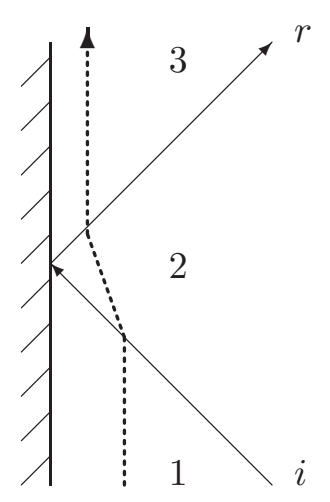

Figure 5.2: Planar shock reflection off a solid surface. The dotted line denotes a particle path.

\subsubsection{General observations of shock-induced collapse}

In order to illustrate the general flow features, shock-induced collapse for a baseline case with $p_{s} / p_{o}=353, H_{o} / R_{o}=2.0, \sigma / R_{o}=135$ is considered. In physical parameters, this corresponds to a $35 \mathrm{MPa}$ shock impacting a $50 \mu \mathrm{m}$ bubble initially located $100 \mu \mathrm{m}$ away from the solid surface. A qualitative description of the events is presented in Figure 5.3. Slices across the computational domain through the centerline show numerical Schlieren (top) and pressure contours (bottom). The location of the interface is highlighted in black in the pressure plot and the dark area on the left of each frame denotes the wall.

The results are very similar to those of Section 4.2.2. When the left-moving shock hits the bubble, an expansion wave is reflected because of the high impedance mismatch, while a weak shock is transmitted (frame 1). The shock then diffracts off the bubble and intersects along the axis. Thereafter, the lithotripter pulse reflects off the wall and effectively doubles the local pressure; the transmitted shock focuses, but not exactly in the center of the bubble (frame 2). The bubble proceeds to collapse non-spherically, while complex wave interactions take place within the bubble (frame 3). The distal side flattens and starts to involute. At collapse, the jet has penetrated the bubble and impacts the distal side; this generates a 
water-hammer shockwave, which propagates spherically outwards (frame 4). The strength of the shock is higher in the direction of the jet. The bubble then takes the shape of a vortex ring and convects towards the wall, while the water-hammer shockwave reflects back onto the bubble (frame 5). As the bubble expands again, the shock interacts with it and reflects back onto the wall as an expansion wave (frame 6).

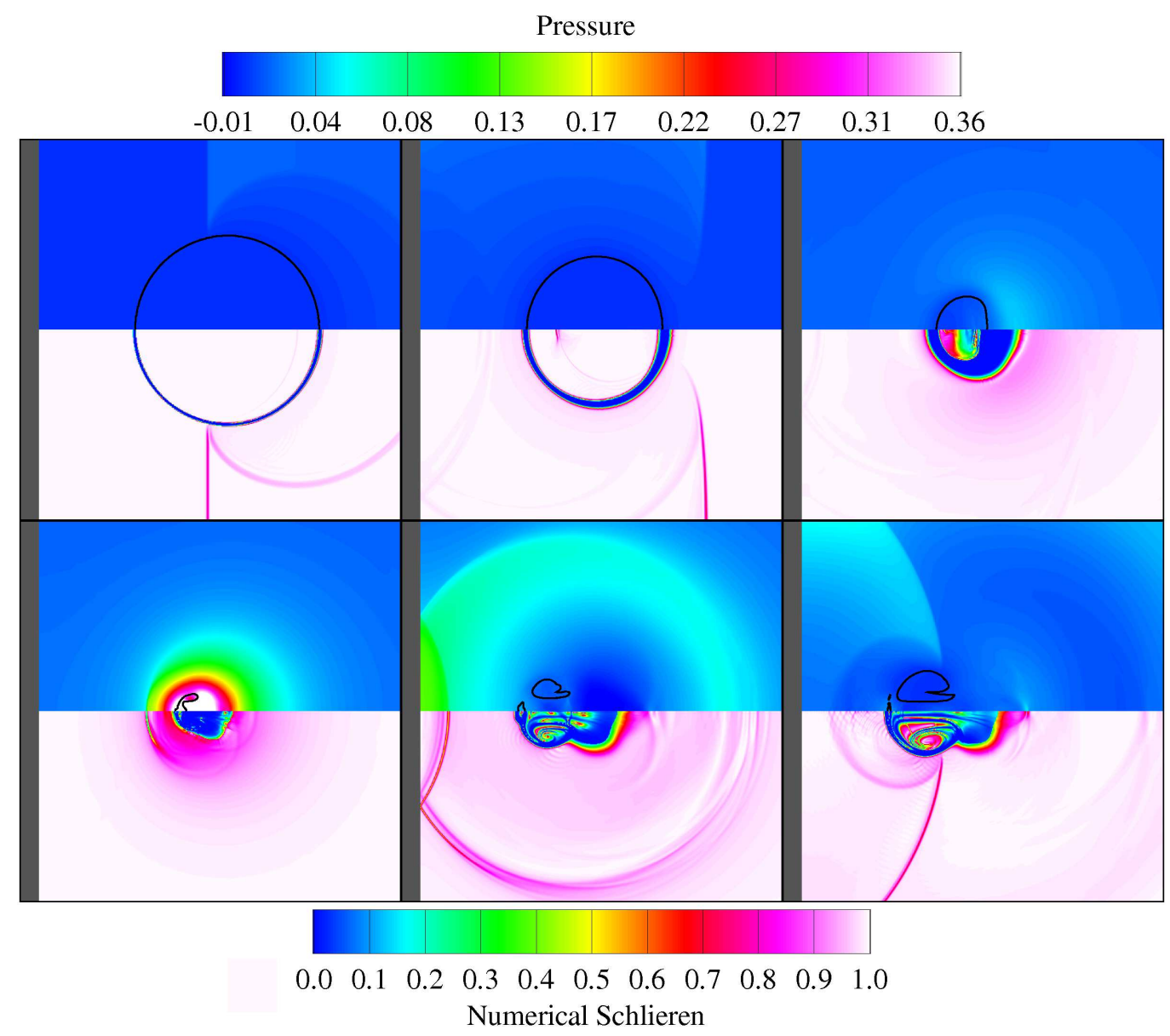

Figure 5.3: Pressure (top) and numerical Schlieren (bottom) contours for shockinduced collapse in SWL $\left(p_{s} / p_{o}=353, H_{o} / R_{o}=2.0, \sigma / R_{o}=135\right)$ at $t c_{L} / R_{o}=$ $5.12,9.77,11.9,12.8,14.0,15.3$. 


\section{Bubble dynamics}

To better understand the bubble dynamics, the history of the bubble volume, stand-off distance, jet velocity and velocity of the distal side are plotted in Figure 5.4. After the passage of the shock $\left(t c_{L} / R_{O} \approx 4\right)$, the bubble begins to collapse. In the initial stages, the collapse is slow, as seen in the early migration of the bubble towards the wall and increase in jet velocity. The external shock reaches the distal bubble side at $t c_{L} / R_{o} \approx 6$, inducing the distal side to move. In the latter stages, high interfacial velocities are achieved; the bubble collapses to a very small size and accelerates towards the wall. The jet eventually impacts the distal side, causing a large deceleration of the interface; this occurs slightly before the bubble reaches its minimum volume.
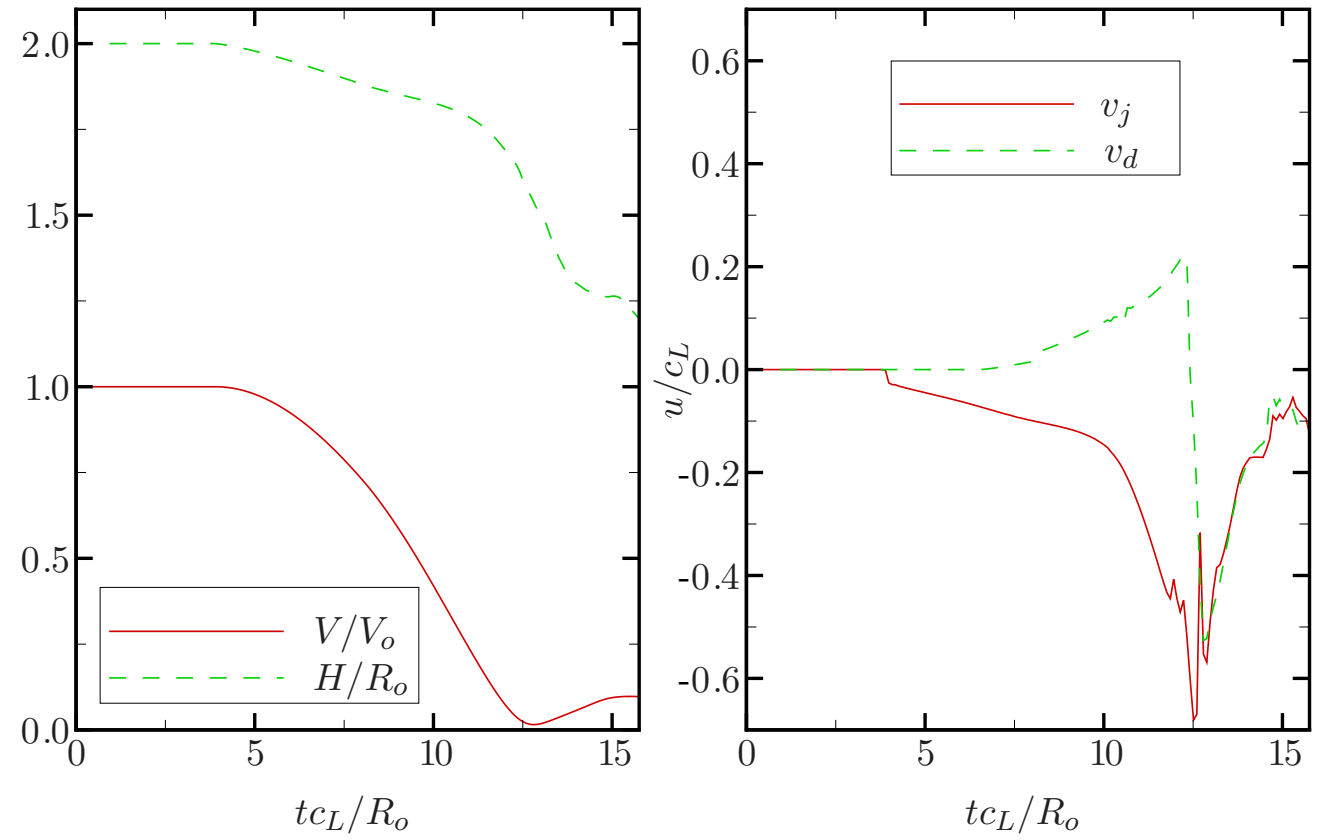

Figure 5.4: History of the bubble volume and displacement (left) and velocity of the jet and of the distal side (right) for shock-induced collapse in SWL $\left(p_{s} / p_{o}=353\right.$, $\left.H_{o} / R_{o}=2.0, \sigma / R_{o}=135\right)$.

Upon the impact of the jet onto the distal side of the bubble, a water-hammer pressure is generated. For the impact of a liquid jet onto a liquid surface, the 
water-hammer equation simplifies to

$$
p_{w h}=\frac{\rho_{L} c_{L}\left|v_{j}-v_{d}\right|}{2}
$$

where $v_{j}$ is the jet velocity $\left(v_{j} \approx 1120 \mathrm{~m} / \mathrm{s}\right)$ and $v_{d}$ is the velocity of the distal side $\left(v_{d} \approx 364 \mathrm{~m} / \mathrm{s}\right)$. The local sound speed and density at the moment of impact are $1330 \mathrm{~m} / \mathrm{s}$ and $1400 \mathrm{~kg} / \mathrm{m}^{3}$, so that the computed water-hammer pressure is 1.5 GPa. In the simulations, the local pressure at the jet is $1.8 \mathrm{GPa}$, giving reasonable agreement. This analysis illustrates that very high velocities and pressures occur during the process. Comparisons with experimental findings are provided for the velocity in Section 5.2.2.

\section{Wall pressure}

The wall pressure is an important quantity indicative of the damage potential of bubble collapse. Figure 5.5 shows the history of the wall pressure for different locations along the wall and pressure profiles along the wall. First, the lithotripter pulse hits the wall at $t c_{L} / R_{o} \approx 7$. The pressure along the centerline $\left(r / R_{o}=0\right)$ is slightly lower and delayed compared to other locations because, when the initial pulse impacts the bubble, a portion of the wave is reflected. In other words, the bubble shields the wall by an amount that depends on $H_{o} / R_{o}$. The shock then reflects off the wall and impacts the bubble again. Because of the impedance mismatch, the amplitude inverts, so that, upon the interaction with the resulting expansion wave, the wall pressure decreases at $t c_{L} / R_{O} \approx 9$. The large pressure rise then observed is caused by the water-hammer shock generated upon collapse; hence, the pressure due to bubble collapse is thus much larger than that due to the pulse. Later in the wall pressure history, negative pressures (tension) are achieved due to the reflection of the water-hammer shock onto the bubble and the subsequent inversion in the amplitude.

Similarly to Section 4.5.4, the wall pressure obeys Equations 4.39 and 4.40. Equation 4.39 is the dashed curve in Figure 4.48, which matches the computations 

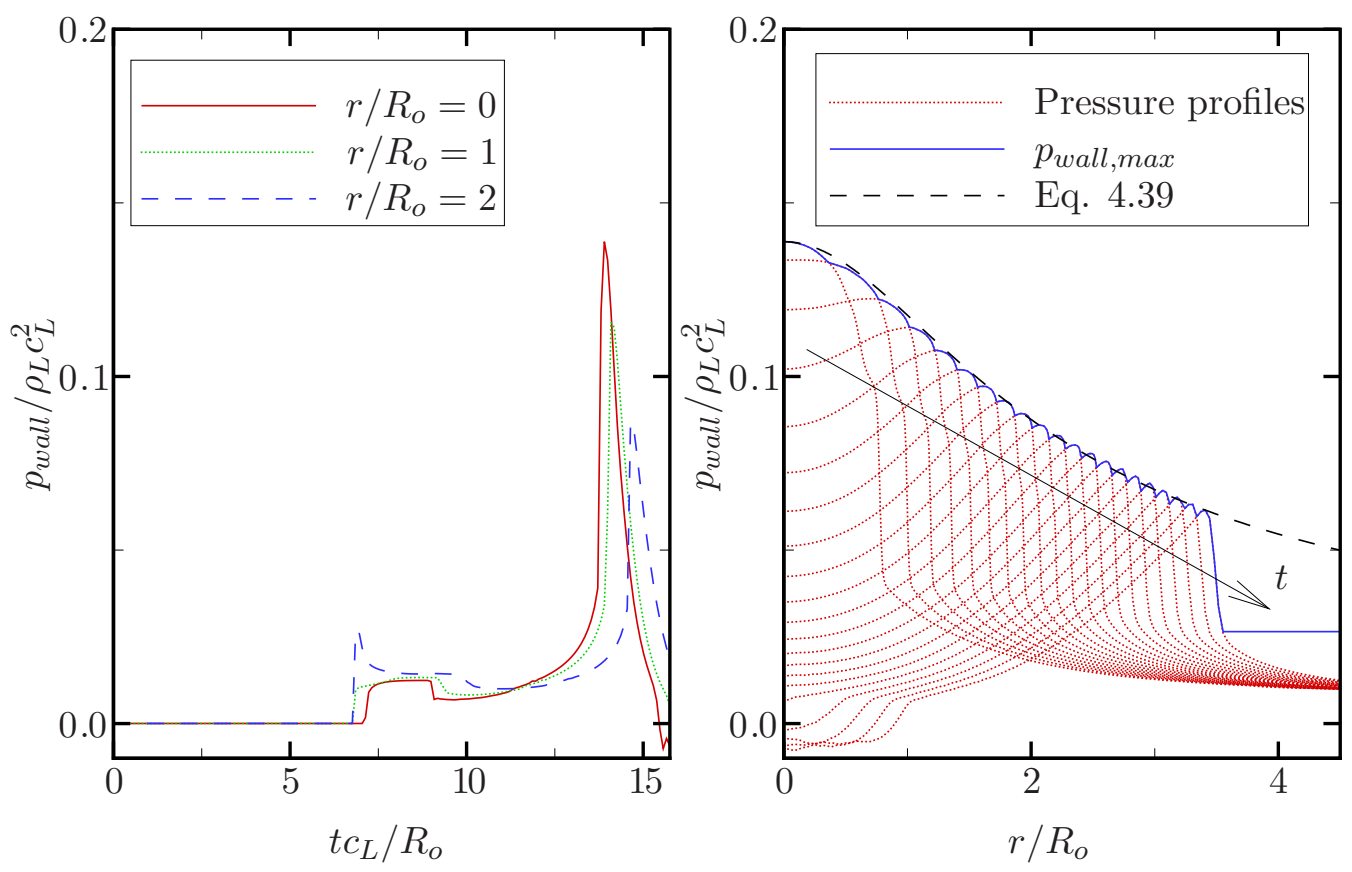

Figure 5.5: History of the wall pressure (left) and pressure profiles along the wall (right) for shock-induced collapse in SWL $\left(p_{s} / p_{o}=353, H_{o} / R_{o}=2.0, \sigma / R_{o}=\right.$ $135)$.

very closely. Using Equation 4.40, the radius of the area over which the wall pressure is larger than that of the pulse is $L_{r} / R_{o} \approx 10$. Thus the area over which the pressure due to bubble collapse is larger than that of the lithotripter pulse is 100 times larger than the projected area of the bubble. Thus, although bubbles undergoing shock-induced collapse are initially small $\left(R_{o} \approx 10 \mu \mathrm{m}\right)$ and collapse to an even smaller size, the area over which they act is much larger $\left(R_{\text {area }} \approx 100\right.$ $\mu \mathrm{m})$.

\subsubsection{Damage potential of shock-induced collapse in SWL}

As a measure of the damage potential of shock-induced collapse in SWL, the maximum wall pressure generated by bubble collapse is considered in the following parametric study. The dependence initial stand-off distance and pulse width is studied. The dependence on the pulse amplitude is almost identical to the results 
in Section 4.5.5 and is therefore omitted.

\section{Dependence on the initial stand-off distance}

The distance at which bubbles are likely to cause damage is of great importance for practical purposes. The dependence of the wall pressure along the centerline on the stand-off distance for $p_{s} / p_{o}=353$ and $\sigma / R_{o}=135,1350$ is shown in Figure 5.6 to illustrate the effect of small and large isolated bubbles in SWL (holding $\sigma$ fixed). Because the bubble migrates towards the wall, the stand-off distance at collapse is considered, since this is when the shockwave is emitted. The same number of computational points are used across the bubble for each $\sigma / R_{o}$. Because the extent of the potential damage scales with the initial bubble radius, the pressure is averaged over the first ten cells in the case $\sigma / R_{o}=1350$, so that the area over which the pressure is recorded is the same in both cases.

As expected, bubbles close to the wall generate a higher pressure (up to 2.4 $\mathrm{GPa}$ ), which decays as $1 / r$ as remarked in the previous section. In addition, for the range of $H_{o} / R_{o}$ considered here, the wall pressure along $r=0$ is much higher than that of the incoming pulse. As noted in Chapter 4 , bubbles with $\sigma / R_{o} \rightarrow 1350$ that collapse within approximately $8 R_{o}$ generate a pressure higher than that of the incoming pulse. The results show that the smaller bubble generates higher wall pressures; however, the extent of the damage scales with the initial radius. This phenomenon is due to the fact that the high pressure of the compressive part of the pulse is exerted over a longer time for a bubble that is small compared to the pulse width. As a generalization, the results indicate that the collapse of a bubble that is large compared to the pulse width (e.g., cavitation bubble) is much more gentle than that of a small bubble (e.g., gas nucleus). The dependence of the bubble dynamics on the pulse width is investigated in the next section. Experiments have measured wall pressures up to approximately $11 \mathrm{MPa}$ for shock-induced collapse (Shima et al., 1984). 


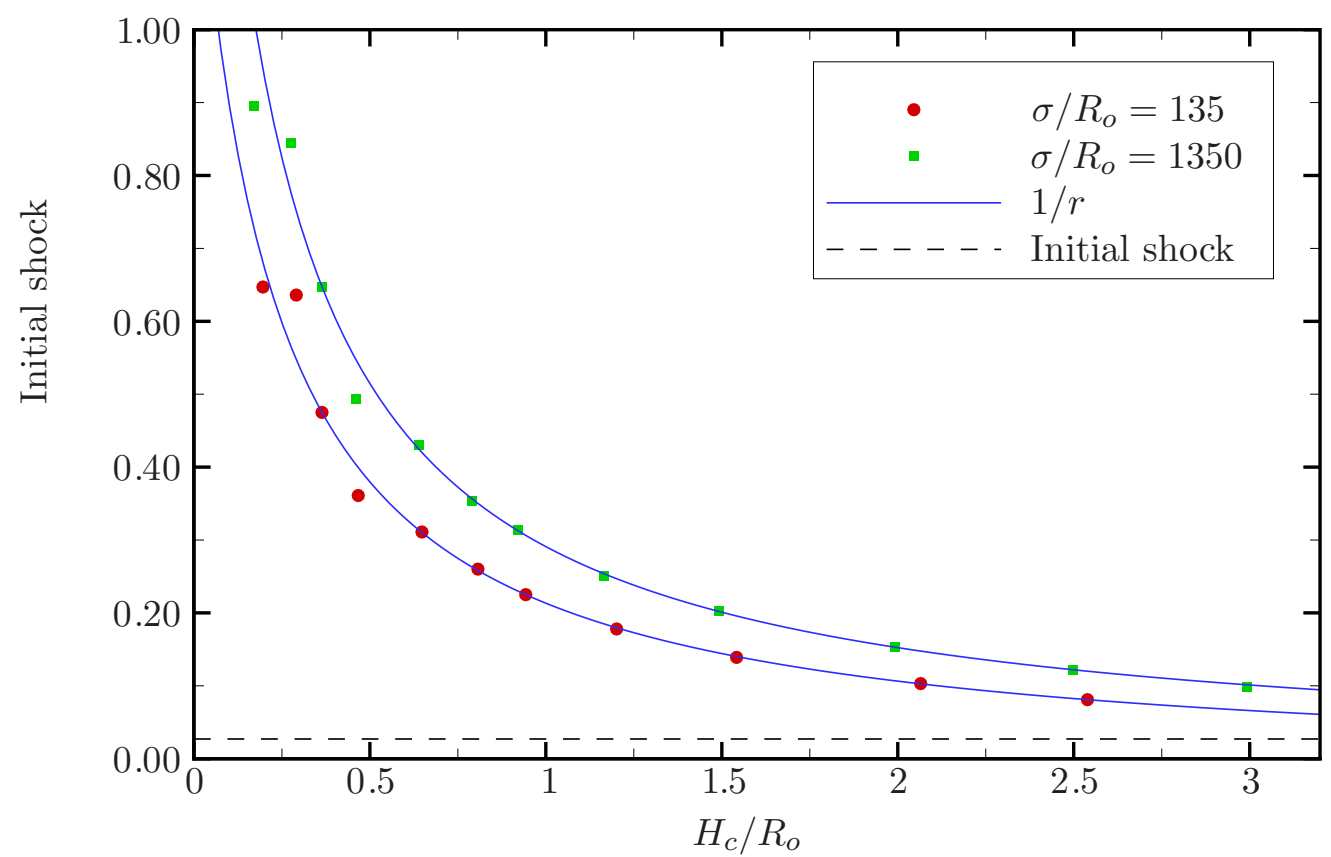

Figure 5.6: Wall pressure along the centerline as a function of the initial stand-off distance for two different bubble sizes $\left(p_{s} / p_{o}=353\right)$.

\section{Dependence on the pulse width}

The main difference between the shock-induced collapse studied in the previous chapter and that in SWL is the finite width of the pulse. As shown in Figure 1.4, the pulse in SWL has a width, $\sigma$, so that the ratio, $\sigma / R_{o}$, is an important parameter. Changing this quantity leads to two possible interpretations: a variable pulse width (which is a property of the lithotripter) or a variable initial bubble radius (which depends on the bubble population). The limit, $\sigma / R_{O} \rightarrow \infty$, corresponds to the previously studied shock-induced collapse, which consits of a stepwise change in pressure. Table 5.1 lists the range of pulse widths and initial bubble radii representative of SWL conditions for shock-induced collapse.

In order to understand the effect of the pulse width in SWL, Figure 5.7 shows the collapse time and the bubble displacement, Figure 5.8 depicts the maximum interfacial velocity (jet and distal side) and the water-hammer pressure for free-field collapse with $p_{s} / p_{o}=353$, Figure 5.9 shows the bubble pressure and normal- 
Table 5.1: Representative values of the pulse width and initial bubble radius for an electro-hydraulic lithotripter.

\begin{tabular}{|l|c|c|}
\hline$\sigma / R_{o}$ & $\sigma\left(\right.$ for $\left.R_{o}=10 \mu \mathrm{m}\right)$ & $R_{o}($ for $\sigma=6.75 \mathrm{~mm})$ \\
\hline 67.5 & $0.675 \mathrm{~mm}$ & $100 \mu \mathrm{m}$ \\
\hline 135 & $1.35 \mathrm{~mm}$ & $50 \mu \mathrm{m}$ \\
\hline 337.5 & $3.70 \mathrm{~mm}$ & $20 \mu \mathrm{m}$ \\
\hline 675 & $6.75 \mathrm{~mm}$ & $10 \mu \mathrm{m}$ \\
\hline 1350 & $13.5 \mathrm{~mm}$ & $5 \mu \mathrm{m}$ \\
\hline
\end{tabular}
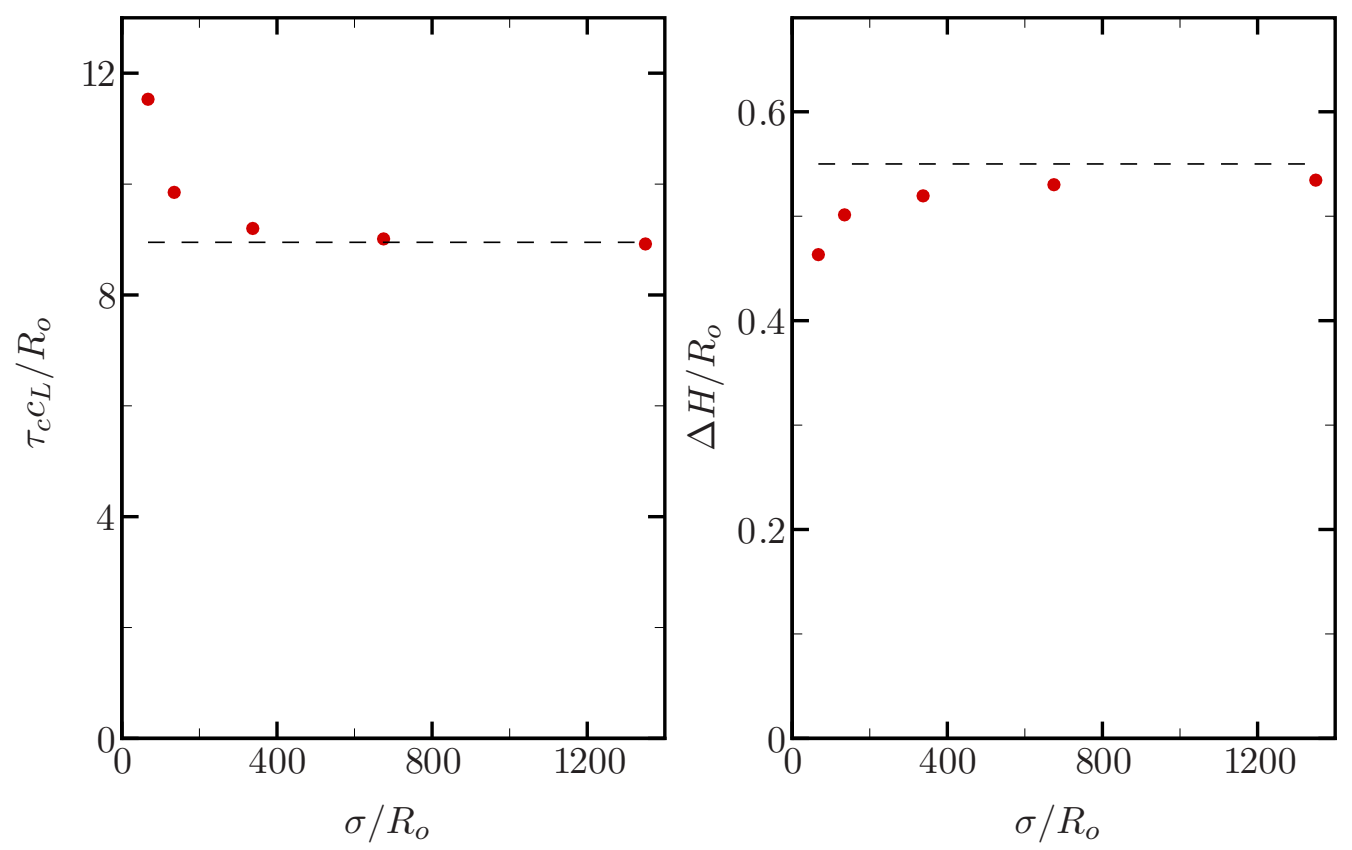

Figure 5.7: Collapse time as a function of the pulse width for free-field shockinduced collapse in SWL $\left(p_{s} / p_{o}=353\right)$. 
ized radiated energy at collapse, and Figure 5.10 depicts the maximum circulation and circulation after the transmission of the internal shock as a function of the non-dimensional pulse width. The dashed lines represent the limiting case of $\sigma / R_{o} \rightarrow \infty$ (i.e., a stepwise increase in pressure, followed by no expansion). For the parameters considered in the present study, the collapse time occurs before the arrival of the negative tail of the pulse (at $t c_{L} / R_{o} \approx 23$ ). Thus, the dynamics of collapse are only affected by the compressive portion of the pulse.
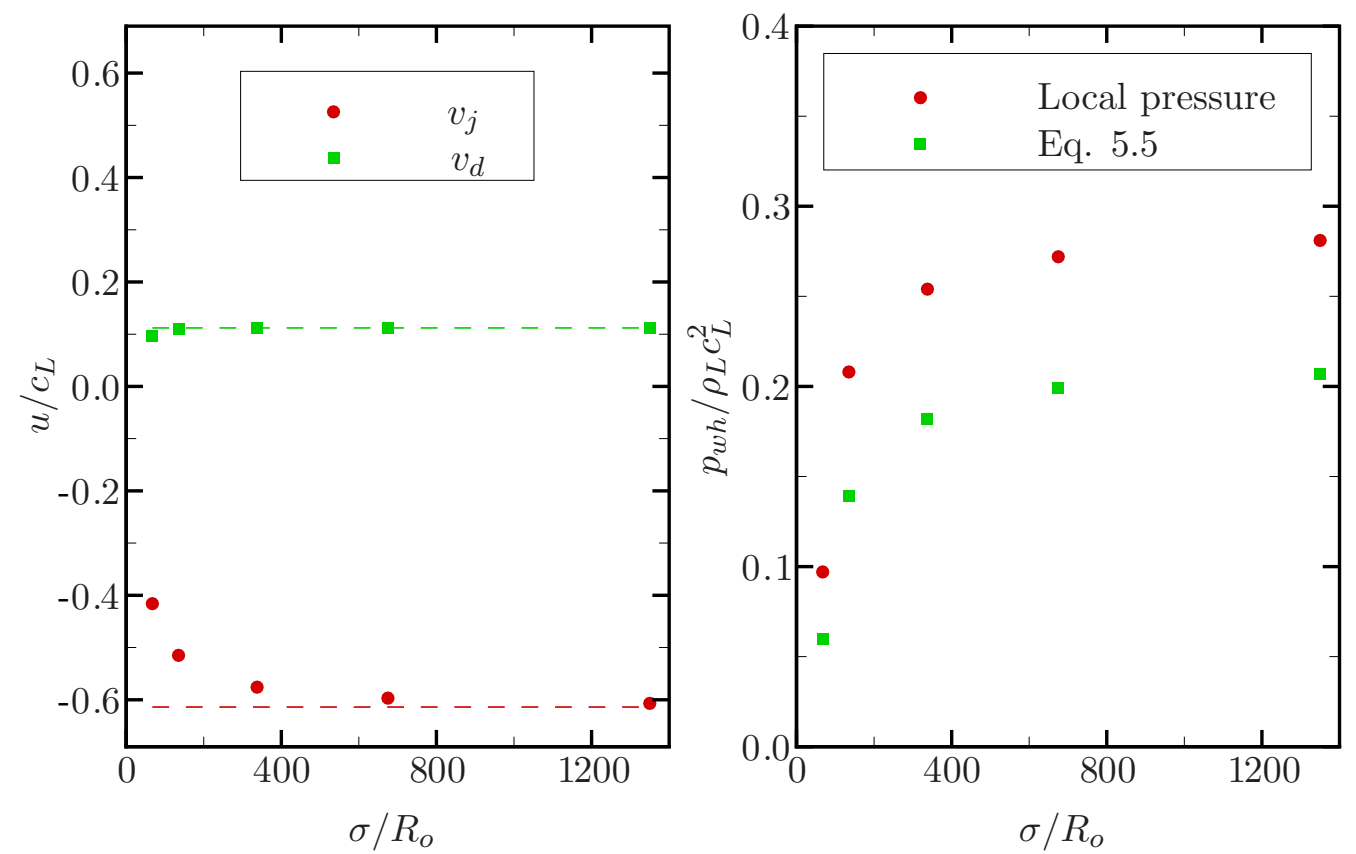

Figure 5.8: Maximum velocity of the jet and of the distal side (left) and measured and computed water-hammer pressure (right) as a function of the pulse width for free-field shock-induced collapse in SWL $\left(p_{s} / p_{o}=353\right)$.

The results show that the dynamics are sensitive to the pulse width. As the pulse width is increased, the behavior of the bubble tends to that resulting from the interaction with a shockwave that has infinite width (denoted by the dashed line); the data for $\sigma / R_{o}=675$ are already close to the asymptotic value.

The behavior of the wall pressure in shock-induced collapse near a solid surface follows a similar trend, as seen in Figure 5.11, though the asymptote is achieved 

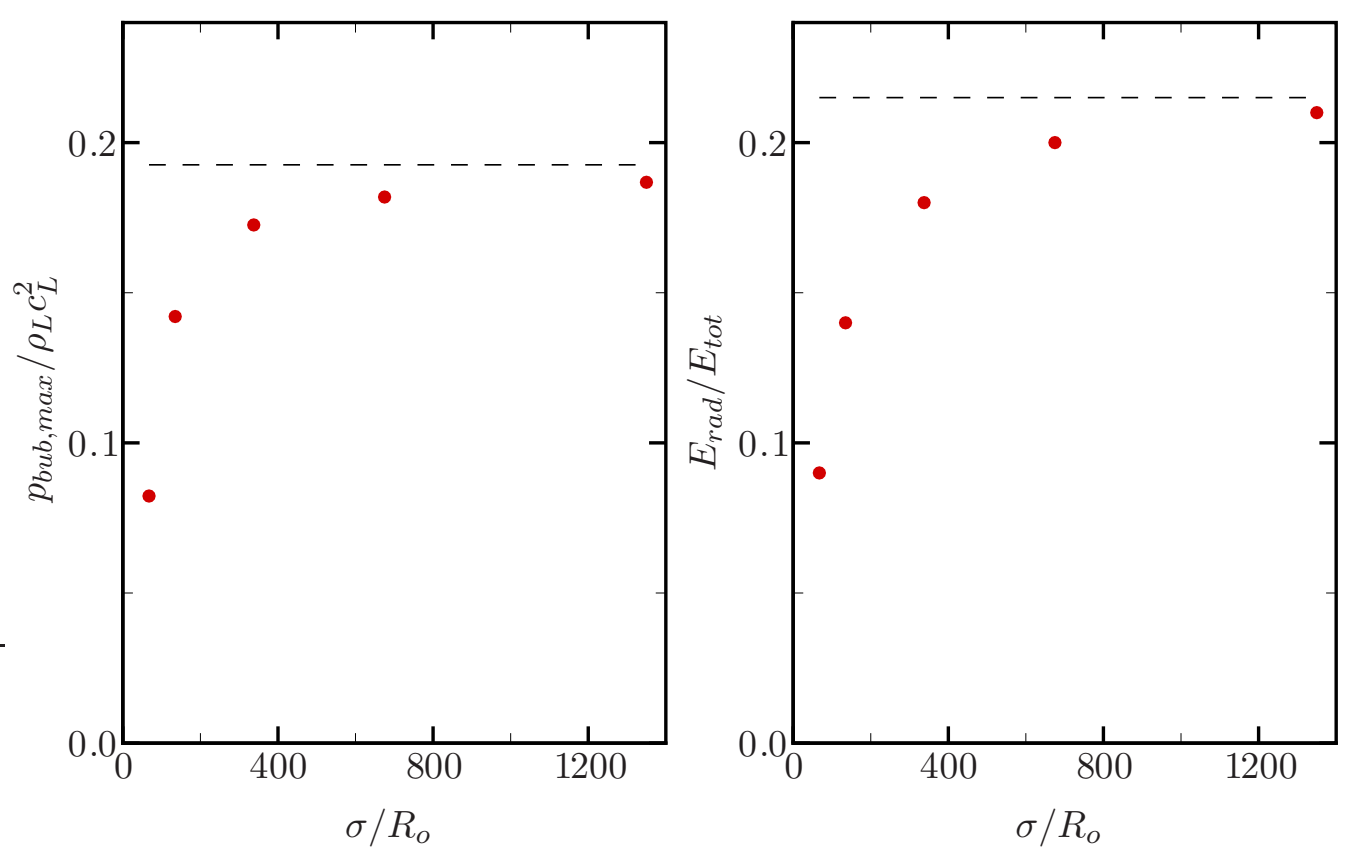

Figure 5.9: Bubble pressure and normalized radiated energy at collapse as a function of the pulse width for shock-induced collapse in SWL $\left(p_{s} / p_{o}=353\right)$.

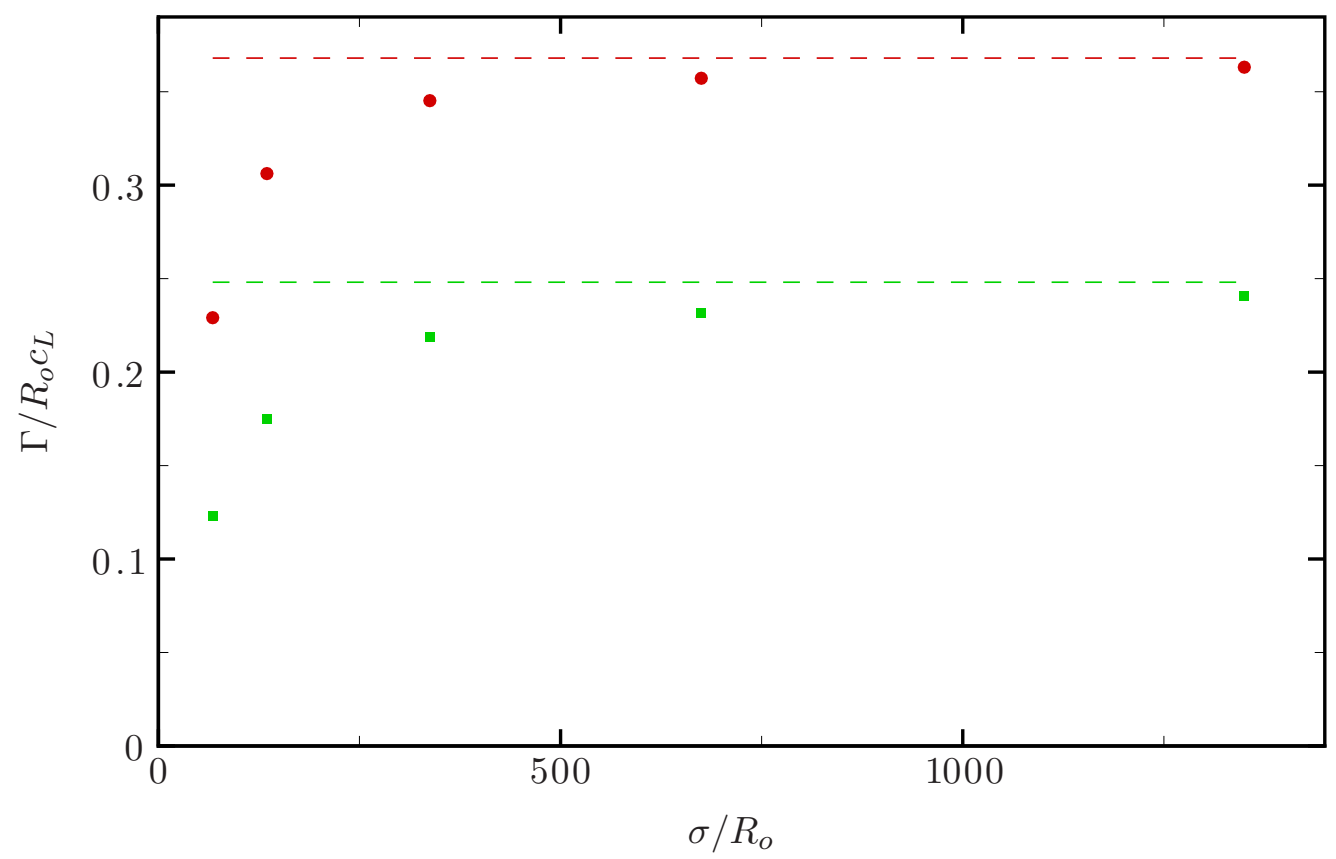

Figure 5.10: Circulation of the transmitted shock and maximum circulation as a function of the pulse width for shock-induced collapse in SWL $\left(p_{s} / p_{o}=353\right)$. 
for larger values of $\sigma / R_{o}$. This phenomenon may be understood by the fact that the pulse subjects the bubble to a high pressure over a shorter time when $\sigma / R_{o}$ is small, as shown schematically in Figure 5.12. An alternate view is to hold $\sigma$ fixed, so that the bubble radius is the variable, as discussed previously.

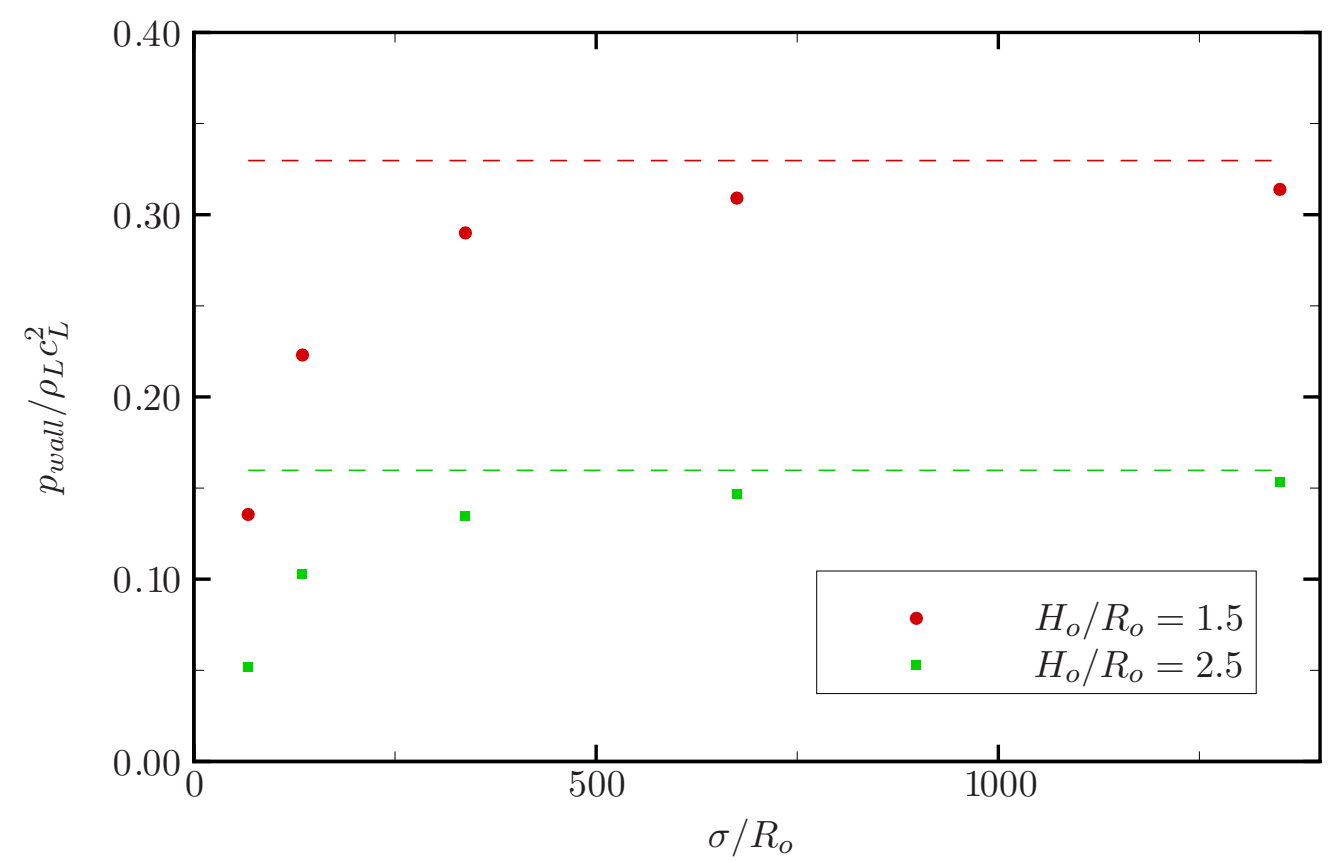

Figure 5.11: Wall pressure along the centerline as a function of pulse width for different initial stand-off distances in SWL with $p_{s} / p_{o}=353$.

In experiments of shock-induced collapse of a gas bubble near an aluminum foil (Philipp et al., 1993), a pressure ratio of $p_{s} / p_{o} \approx 650$ is used. The pulse width in this case is $\sigma \approx 10 \mathrm{~mm}$ and the range is $\sigma / R_{o}=11.4-20.8$, which corresponds to large bubbles. A trend similar to the present results is observed for the collapse time, which falls in the range $\tau_{c} c_{L} / R_{O}=6.51-9.36$, as a function of the pulse width (or initial bubble radius). The collapse time decreases with increasing pulse width, eventually asymptoting to the value represented by a stepwise increase in pressure. Jet velocities of approximately $600-700 \mathrm{~m} / \mathrm{s}$ are achieved in this range of $\sigma / R_{o}$; the present simulations yield a jet velocity of $660 \mathrm{~m} / \mathrm{s}$ for $p_{s} / p_{o}=353$ and $\sigma / R_{o}=67.5$. Although the parameters are different, similar orders of magnitude 


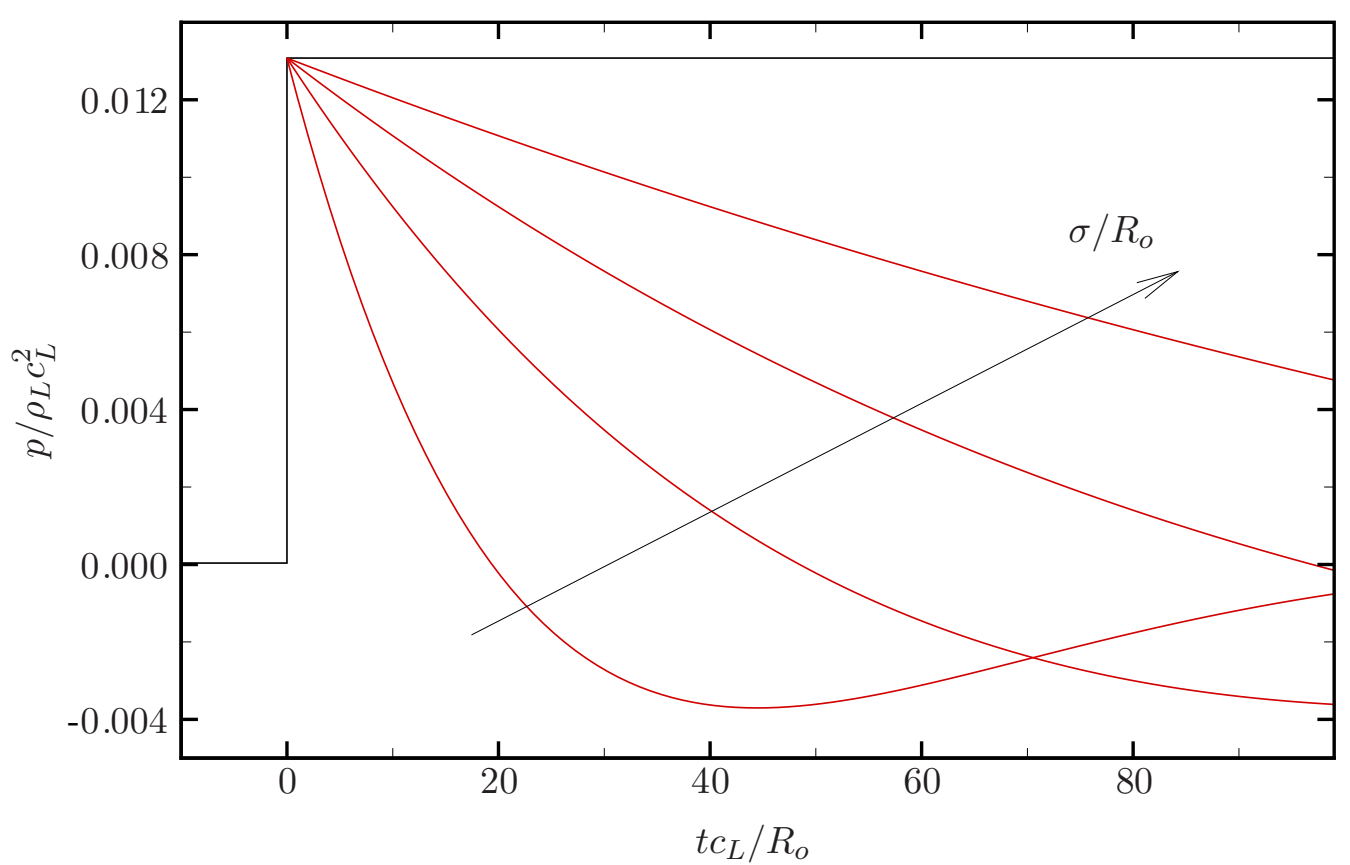

Figure 5.12: Waveform for increasing pulse widths.

and trends are achieved in the simulations. In free-field experiments with lower pulse amplitude (Ohl \& Ikink, 2003) $\left(p_{s} / p_{o}=100-200\right)$, jet velocities up to 150 $\mathrm{m} / \mathrm{s}$ are achieved for $R_{o}=50 \mu \mathrm{m}$, while jet velocities of $360-700 \mathrm{~m} / \mathrm{s}$ are achieved in the simulations. In these experiments however, the rise time is much slower than in the simulations, and measured values constitute lower bounds limited by the resolution of the laboratory equipment.

\subsubsection{Rayleigh collapse in SWL}

The damage potential of shock-induced collapse has been considered so far. The second type of collapse that occurs in SWL is Rayleigh collapse, where a vapor bubble collapses due to the pressure difference across the interface, $\Delta p \approx p_{a t m}-p_{v}$. The present discussion focuses on how the wall pressures generated by Rayleigh collapse compare to those generated by shock-induced collapse in SWL.

Experiments (Tomita \& Shima, 1986) show that wall pressures up to $11 \mathrm{MPa}$ are achieved for $H_{o} / R_{o} \gtrsim 1$. In these experiments, large bubbles $\left(R_{o}=3.5,5.1\right.$ 
$\mathrm{mm}$ ) are generated using electrodes, and the radius of the hydrophone is $2.775 \mathrm{~mm}$. The pressure measurements constitute an average over the area of the hydrophone, so that a higher local pressure is expected to be achieved over a smaller area. Numerical simulations with the same parameters were carried out. However, wall pressures four to five times smaller were obtained $(\sim 2.5-3 \mathrm{MPa})$ at similar stand-off distances, for the reasons explained in Section 4.5.4.

Nevertheless, interesting information can be obtained. Taking as a specific case the results for $H_{o} / R_{o}=1.05$, the wall pressure averaged over an area of radius 2.775/5.1 is 2.2 MPa. However, the maximum local pressure measured along the wall in the simulations (which occurs at $r=0$ ) is $2.6 \mathrm{MPa}$. This seems to indicate that the local wall pressure achieved in an isolated Rayleigh collapse may not be larger than the pressure of the incoming lithotripter pulse at all.

Experiments of shock-induced collapse of air bubbles were carried out Tomita et al. (1983), using a shock amplitude of $5 \mathrm{MPa}$. Wall pressures of approximately $10 \mathrm{MPa}$ were obtained with similar hydrophones, which may have led one to believe that the pressures achieved in shock-induced collapse are similar to those resulting from Rayleigh collapse. However, the shock amplitudes in SWL are five to ten times larger and, according to Section 4.5.5, the resulting wall pressure due to bubble collapse increases linearly.

Thus, wall pressures higher than those of the incoming pulse are generated in shock-induced collapse. Based on current experiments and simulations, it is not clear that Rayleigh collapse leads to wall pressures higher than $35 \mathrm{MPa}$ (i.e., the pulse amplitude), though ample evidence exists that the pitting of solid surfaces may occur due to the collapse of cavitation bubbles Philipp \& Lauterborn (1998). On the other hand, when subjected to a $35 \mathrm{MPa}$ shock, a wall pressure on the order of $1 \mathrm{GPa}$ may be generated locally upon collapse. Therefore shock-induced collapse has a greater potential for damage in SWL than Rayleigh collapse, even though the bubbles are typically smaller than in Rayleigh collapse.

Though shock-induced collapse therefore appears to bear a prominent role in the stone comminution mechanism, the high pressures generated during such a 
process may lead to negative effects in SWL. For instance, if the bubbles collapse in unwanted ares (e.g., near tissue), significant trauma may occur upon their shockinduced collapse (Evan et al., 2002).

\subsection{Stone comminution}

\subsubsection{Spallation and cavitation}

One of the important stone comminution mechanisms is the wave propagation within the stone, such as spallation (Gracewski et al., 1993; Xi \& Zhong, 2001) or constructive interference of shear waves (Cleveland \& Sapozhnikov, 2005). Because kidney stones are principally made of calcium (Zhong et al., 1993), they are brittle, so that they are more likely to fail in tension. In spallation, lithotripter pulses propagate through the stone and, upon reflection from the distal side of the stone, invert their amplitude. For stones greater than a critical size, this large negative pressure superposes over the tensile part of the incoming pulse, thus creating a magnified tensile region near their posterior end. In addition, the shockwave propagating in the water generates shear waves, which may interfere constructively within the stone to generate high tension. This latter phenomenon greatly depends on the geometry of the stone.

Another important stone comminution mechanism is cavitation erosion. After the passage of the tensile part of the pulse, bubbles gather along the surface of the stone; there, they grow and collapse, thereby generating shockwaves (Xi \& Zhong, 2001; Pishchalnikov et al., 2003). When a single bubble collapses near a solid surface, a re-entrant jet directed towards the surface forms and penetrates the bubble (Plesset \& Chapman, 1971). The direct impact of this jet onto the surface can cause significant damage (Tomita \& Shima, 1986; Philipp \& Lauterborn, 1998) and has been regarded as the primary cause of cavitation erosion in SWL (Crum, 1988).

The individual effect of each of the two mechanisms described above is difficult to assess. (Zhu et al., 2002) conducted experiments seeking to isolate each effect. 
When cavitation is suppressed, it is shown that the stress waves in the stone are strong enough to cause spallation, due to the superposition of the tensile waves reflected off the end of the stone with the negative part of the incoming pulse. However, this occurs only if the stone is larger than half the wavelength of the incoming pulse; smaller stones do not fail in this fashion. On the other hand, if only cavitation is allowed, the stones do not spall, but pitting is observed on the anterior surface. When both cavitation and shockwaves are allowed, both spallation and pitting occur, and the process is far more efficient.

\subsubsection{A comminution mechanism for medium-sized stones}

The present results show that a large localized pressure is generated along the stone surface due to bubble collapse, especially in the case of shock-induced collapse. This high pressure explains the pitting along the proximal surface of the stone (Philipp \& Lauterborn, 1998). In addition, the pressure wave generated by bubble collapse propagates through the stone and, after reflecting upon the stone end, inverts its amplitude. Thus, a phenomenon similar to spallation may occur. Furthermore, the propagation of this pressure wave along the edge of the stone may generate shear waves. However, because the pressure decays as $1 / r$, such effects would be more important for smaller stones. This phenomenon may explain why the combined effect of shock propagation within the stone and cavitation leads to the most efficient stone comminution rate (Zhu et al., 2002).

To verify this hypothesis, the hydrodynamic simulations discussed throughout this thesis were prescribed as an input for the propagation of elastic waves within the stone, using the code of Cleveland \& Sapozhnikov (2005). In this model, the kidney stone is assumed to behave as a linear, isotropic, elastic solid. Thus, Newton's second law,

$$
\frac{\partial v_{i}}{\partial t}=\frac{1}{\rho_{o}} \frac{\partial \tau_{i j}}{\partial x_{j}}
$$

and Hooke's law,

$$
\frac{\partial \tau_{i j}}{\partial t}=\lambda \delta_{i j} \frac{\partial v_{k}}{\partial x_{k}}+\mu\left(\frac{\partial v_{i}}{\partial x_{j}}+\frac{\partial v_{j}}{\partial x_{i}}\right)
$$


are solved using a time-domain finite difference method (FDTD). Here, $v_{i}$ is the velocity in the $i$ th direction, $\tau_{i j}$ is the stress tensor, $\delta_{i j}$ is the Kronecker delta function, $\rho_{o}$ is the density, and $\lambda$ and $\mu$ are the Lamé coefficients.

For the elastic waves simulations, the computational domain consists of a kidney stone immersed in water. Values of $\rho_{o}, \lambda$ and $\mu$ representative of water and kidney stones are used. The pressure along the stone surface is specified from the hydrodynamic simulations as a time-varying boundary condition for the elastic waves simulations. The wave propagation within the stone does not affect the wall pressure; in other words, there is only a one-way coupling between the hydrodynamics and the wave propagation. The full coupling between fluid dynamics and solid mechanics presents many difficulties and constitutes a field of studies in itself (Wardlaw \& Luton, 2000; Dowell \& Hall, 2001).

Contours of the maximum tensile stress over the course of the simulation are plotted in Figure 5.13 and 5.14 for large $\left(R_{\text {stone }}=2 \mathrm{~mm}\right)$ and small $\left(R_{\text {stone }}=1\right.$ $\mathrm{mm}$ ) spherical stones. The units for the stresses are $\mathrm{MPa}$, and the white line denotes the stone outline. In these figures, the bubble collapses below the stone and is initially located at $H_{o} / R_{o}=1.5$, with $R_{o}=100 \mu \mathrm{m}$.

High tensile stresses are observed on the proximal side. The shock generated upon bubble collapse reflects off the stone back onto the bubble. As it subsequently reflects off the bubble, the amplitude of the shock inverts, thereby generating the large tension in the water on the proximal side of the stone. As a result, a large tensile wave is transmitted into the stone and leads to a tension of approximately $100 \mathrm{MPa}$. The tensile stress contours on the proximal side are similar for both stone sizes, because they result from the same bubble collapse.

The shock generated upon collapse propagates through the stone. As it reflects off the distal side, the shock inverts its amplitude and converges along the vertical axis, leading to a high tension (approximately $100 \mathrm{MPa}$ ) on the distal side of the stone. As expected, this effect is more severe for the smaller stone, because the amplitude of the shock strength decays as $1 / r$ within the stone as well.

Thus, bubble collapse leads to damage on the proximal side of the stone due to 


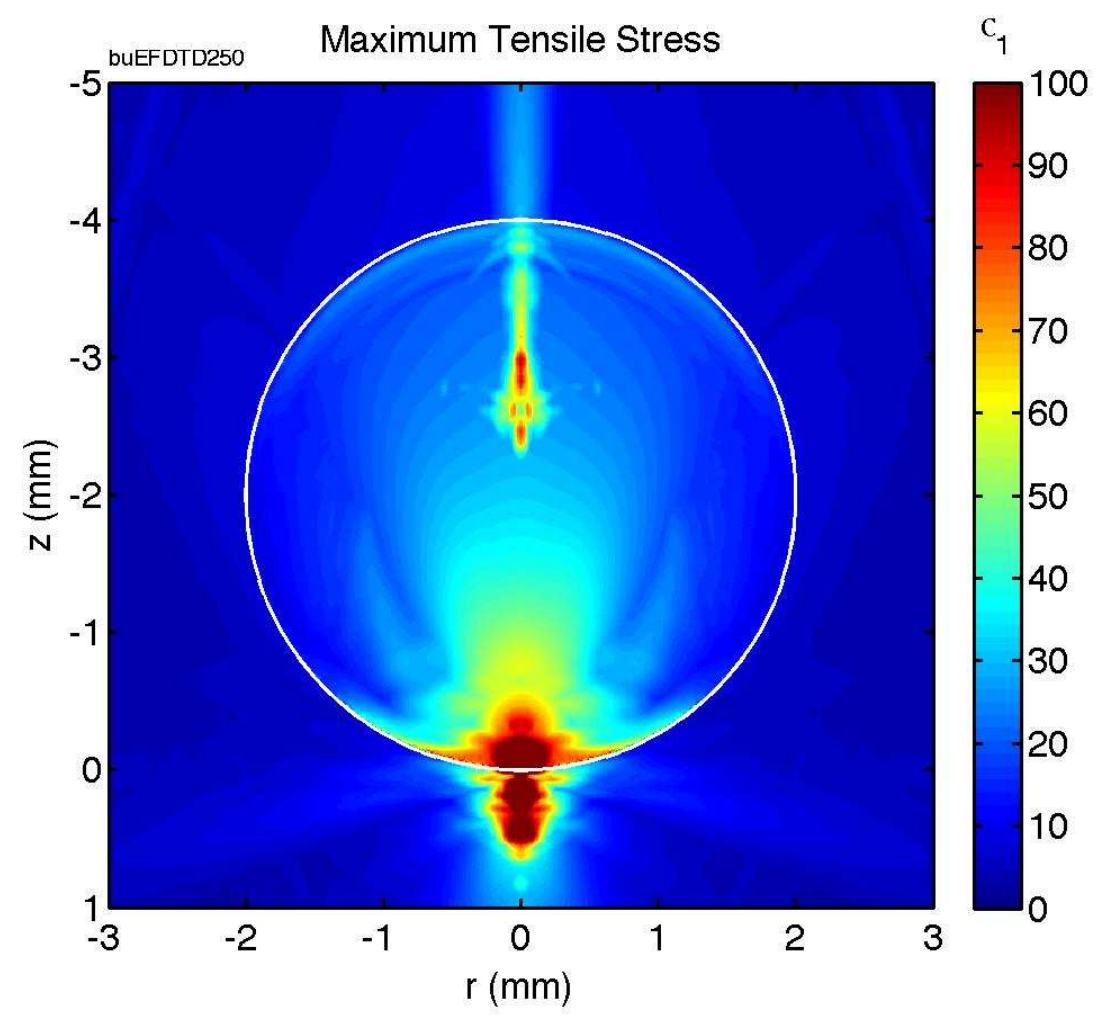

Figure 5.13: Maximum tensile stress (in $\mathrm{MPa}$ ) for a $2 \mathrm{~mm}$ radius stone over the course of the elastic waves simulation.

the high pressure and subsequent tension, as reported in the literature. In addition, the propagation of the pressure wave generated upon collapse leads to spallationlike damage towards the distal end of the stone. Kidney stones typically have tensile strength on the order of $1 \mathrm{MPa}$ (Cohen \& Whitfield, 1993; Lokhandwalla \& Sturtevant, 2000), so that both effects are expected to be important.

Based on this analysis, a more comprehensive picture of stone comminution is painted:

- Spallation/shear waves due to the incoming pulse breaks the stone into smaller bits,

- Spallation due to single-bubble collapse further fragments the resulting pieces, and 


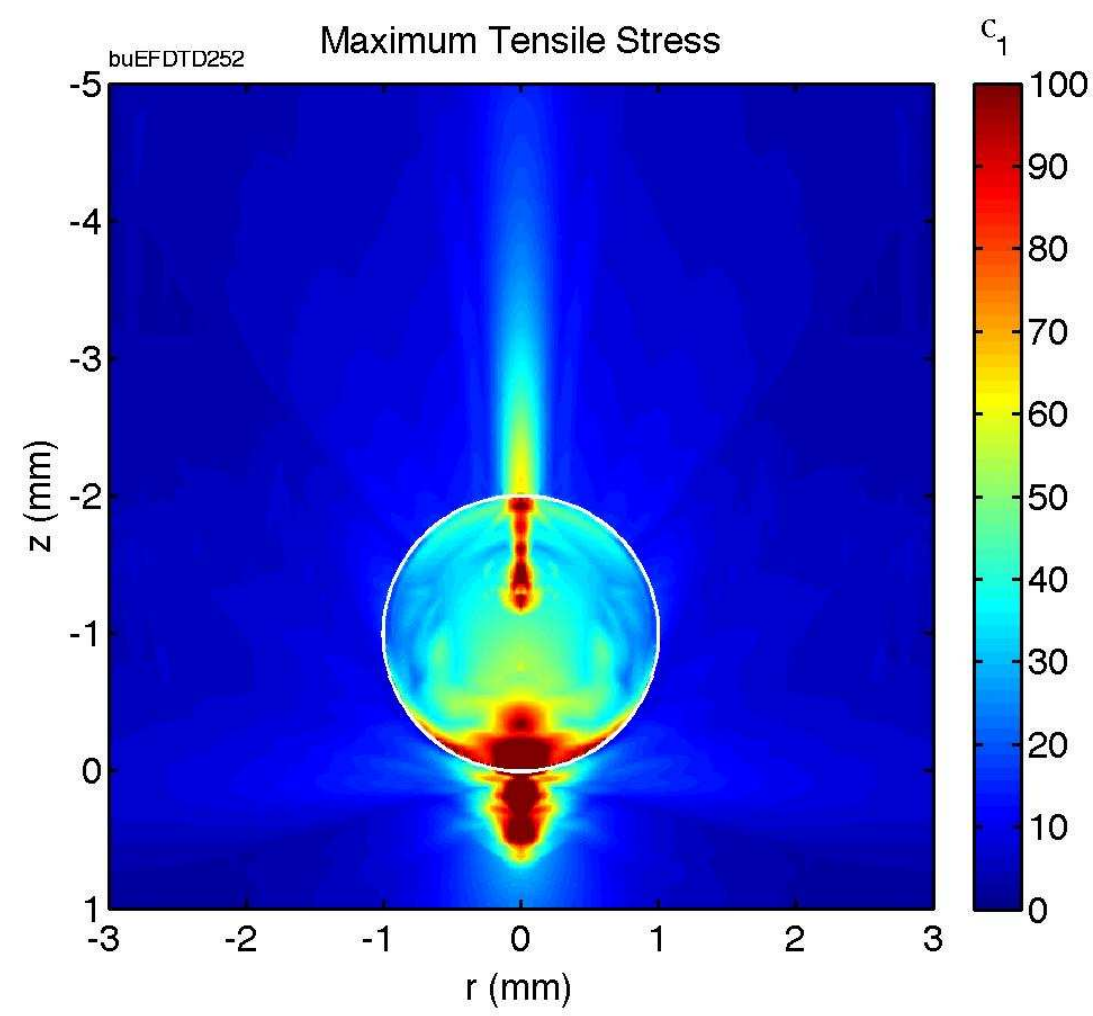

Figure 5.14: Maximum tensile stress for a $1 \mathrm{~mm}$-radius stone over the course of the elastic waves simulation.

- Cavitation erosion grinds the remaining fragments to an even smaller size, while eroding the surface of larger pieces.

\subsection{Summary of the SWL results}

In the present chapter, bubble collapse in the context of shockwave lithotripsy is studied. The main difference with the previously studied shock-induced collapse is that the lithotripter pulse has a finite width. As the width is increased, the behavior tends to that of shock-induced collapse considered previously.

Shock-induced collapse is established as an important comminution mechanism in SWL. It is shown that the area on the wall over which the pressure due to bubble collapse is larger than that of the lithotripter pulse is 100 times larger than the 
projected area of the bubble.

Furthermore, it is shown that bubble collapse in the vicinity of kidney stones may lead to two types of damage. First, the high pressures and tensions generated along the surface may cause pitting. In addition, the reflection and inversion of the amplitude of the pressure wave off the distal side of the stone leads to a high tension near the distal end of the stone. This latter mechanism is likely to be important only for small- to medium-sized stones. 


\section{Chapter 6}

\section{Concluding remarks}

This chapter summarizes the work conducted in the present research and provides an outlook for possible future directions.

\subsection{Summary and conclusions}

In the present work, a new numerical framework was developed to simulate in an accurate and robust fashion compressible multicomponent flows with shocks. This method was then used to conduct a fundamental study of non-spherical bubble dynamics and investigate the potential damage inflicted by bubble collapse in general. The results were then cast in the context of shockwave lithotripsy (SWL), in an attempt to better understand the role of bubble collapse in the stone comminution mechanism.

The numerical method is based on the quasi-conservative shock- and interfacecapturing schemes of Abgrall (1996). The Euler equations, closed by a stiffened equation of state and augmented by appropriate advection equations, are solved. In order to achieve high-order accuracy and avoid interface oscillations, a finite volume WENO formulation was implemented by reconstructing average primitive variables, and the HLLC approximate Riemann solver was extended to the advection equations. The resulting numerical method is oscillation-free for the advection of a material interface and for isolated shock-wave problems; no oscillations are observed in more complex problems, such as multicomponent Riemann problems 
and shock-bubble interactions. Furthermore, mass, momentum and energy are conserved to round-off levels. The numerical method was verified using an extensive series of test problems and thereafter extended to cylindrical coordinates and to a stretched grid in order to compute axisymmetric bubble collapse.

Numerical simulations of Rayleigh collapse and shock-induced collapse, the two main types of collapse in SWL, were carried out either in a free field or near a solid wall. The flow visualizations showed that, during the non-spherical collapse, a re-entrant jet formed towards the wall and/or in the direction of propagation of the shock. The jet eventually impacts the distal side, thus generating a strong water-hammer pressure wave. At this instant, the minimum volume is achieved. For the range of parameters considered in the present study, the water-hammer shock is always generated within the liquid and then propagates radially outwards. The strength of the shock is highest in the direction of propagation of the jet. This phenomenon can be considered a combination of the two previously proposed damage mechanisms: the emission of the gas compression shock (Rayleigh, 1917) and the direct impact of the jet onto the solid surface (Kornfeld \& Suvorov, 1944).

The dynamics of non-spherical bubble collapse were characterized by considering a number of properties of the collapse. The initial motion of the bubble showed good agreement with theoretical predictions based on the corresponding Riemann problem. The collapse time indicated good agreement with theory and experiments. The transient effect of the finite speed of propagation in shock-induced collapse near a wall was highlighted, as the effective pressure doubling driving the collapse is achieved almost immediately for bubbles close to the wall, but much later for bubbles farther away. The bubble is spherical during most of the collapse and becomes non-spherical only late in the latter stages. In addition, it was shown that a bubble migrates towards the wall during collapse, in good agreement with experiments. Jet properties were measured; velocities up to $100 \mathrm{~m} / \mathrm{s}$ in Rayleigh collapse near a wall and up to $1000 \mathrm{~m} / \mathrm{s}$ in shock-induced collapse were achieved. The jet size was measured to show that viscous and surface tension effects are mostly negligible in the present study. 
The re-entrant jet formation mechanism was studied in detail for shock-induced collapse. It was determined that a number of phenomena played a role, such as the growth of non-spherical perturbations, baroclinic vorticity and the transient effects of shock propagation. In Rayleigh collapse near a wall, it is shown that the contraction of the distal side is reduced because expansion waves generated initially decrease the local pressure driving the collapse.

The sequence of shockwave emission was studied. A precursor shockwave is generated by the piston-like motion of the jet, which leads to wave breaking within the bubble. Further shockwaves are emitted due to the compression of the gas within the bubble (gas compression shock) and the impact of the jet onto the distal side (water-hammer shock). The water-hammer pressure was tabulated based on theory and measurements in the simulations; local pressures on the order of 1 GPa are achieved. The jet always impacts the distal side first, so that the waterhammer shock is generated in the liquid. The jet may impact the wall; however, for the range of stand-off distances considered here, the jet velocity at that instant is small.

Prompted by experimental observations, the energy radiated during collapse was studied. It was determined that the acoustical energy radiated during collapse decreases as the collapse becomes more non-spherical, thus leading to longer and larger rebounds in non-spherical cases because of the fact that more energy is retained.

As a measure of damage due to single-bubble collapse, the pressure was computed along a nearby solid surface. For a $35 \mathrm{MPa}$ shock, jet velocities up to several hundreds of meters per second and local wall pressures on the order of $1 \mathrm{GPa}$ were measured locally. The pressure decayed as $1 / r$. It was determined that a bubble collapsing within a distance of $8 R_{o}$ from the wall generates as large a pressure as the incoming shock. In addition, the extent of the region where the pressure is larger than that of the incoming shock can range up to $6 R_{o}$ along the wall for bubbles initially close to the wall. Because bubbles migrated towards the wall during collapse, a higher wall pressure was measured; however, the presence of 
the wall led to a gentler collapse, so that the resulting wall pressure was slightly lower than than expected from the $1 / r$ relationship. It was further shown that the bubble locally shields the wall from the incoming shock.

The results from Rayleigh collapse and shock-induced collapse were cast in the context of SWL. The main characteristic of the lithotripter pulse is its finite

width, so that the collapse depends on the size of the bubble relative to that of the pulse: a bubble large compared to the pulse width leads to a gentler collapse than a smaller one.

Shock-induced collapse is established as an important comminution mechanism in SWL. It is shown that the area on the wall over which the pressure due to the bubble collapse is larger than that of the lithotripter pulse is 100 times larger than the projected area of the bubble. However, if such a collapse occurs in unwanted areas, it has the potential to lead to trauma.

By using the present results as input for elastic waves simulations in kidney stones, it was shown that the pressure waves generated during bubble collapse may lead to damage within the stone, in addition to erosion along its surface. Depending on the geometry, the shock generated by the collapse may reflect as an expansion wave off the end of the stone, thus creating a high tension within the stone. Spherical stones of small to medium sizes are more likely to be affected by this phenomenon, which would explain why the combination of shock propagation within the stone and cavitation is the most efficient means to break kidney stones.

\subsection{Suggestions for future work}

The present work opens new avenues in the study of bubble collapse via numerical simulations. On one hand, extensions of the model and numerical method would allow the implementation of more accurate physical conditions. On the other hand, the present methodology could be used to solve analogous problems. 


\subsubsection{Physical model}

The governing equations can be improved by including additional physical effects, such as viscosity and heat transfer, surface tension and phase change, and turbulence modeling. These features would allow more accurate simulations in certain regions.

The resulting diffusive terms (mass transfer, viscous effect and heat transfer) may be treated in a flux formulation, so that the numerical method does not need to be modified significantly. These terms introduce additional time scales and non-dimensional numbers (Schmidt, Reynolds and Prandtl numbers), so that the CFL condition must be modified to take these into account.

The implementation of surface tension and phase change involves modifications to the model (source terms in the equations of motion) and to the numerical method. The WENO reconstruction and the Riemann solver would have to be extended accordingly. An additional non-dimensional number (Weber number) would account for surface tension, while further modeling in the form of mass and energy conservation equations at the interface would be required for phase change.

The flows resulting from interactions between shockwaves and interfaces are likely to be turbulent. Thus, turbulence modeling is required to account for the energy dissipation at the small scales. However, subgrid-scale modeling in the presence of shockwaves and interfaces is extremely challenging.

\subsubsection{Numerical method}

Although the problems considered in the present work are axisymmetric, in general bubble collapse in SWL is three-dimensional. The two-dimensional Cartesian code can be extended to three dimensions trivially. However, the computational cost would be significantly higher, so that the code would need to be parallelized, e.g., using the Message Passing Interface (MPI). This would require the domain to be decomposed into sub-domains; each processor would then solve the problem on a given sub-domain, given appropriate boundary conditions. 
With regards to the implementation of turbulence modeling for the present problems, it is far from clear how this can be done in a fashion consistent with the current shock-capturing framework, because the numerical dissipation introduced to stabilize the solution near discontinuities may overwhelm the small scales of turbulence. A hybrid methodology where dissipation is introduced near shocks and interfaces may constitute a starting point, though its stability may be an issue.

\subsubsection{Extension to related problems}

Using the present framework, a number of related bubble collapse problems may be solved for general engineering applications or shockwave lithotripsy. Though a more extensive investigation of single-bubble collapse in SWL problems could be carried out (e.g., multiple bubbles, different wall impedances), the following methodology could be applied to underwater explosions, for which the relevant problem configurations are similar.

Problems relevant to the dynamics of bubble clouds and shock propagation in bubbly mixtures include Rayleigh collapse and shock-induced collapse of multiples bubbles in a row. In these fundamental problems, the distance between the bubbles and the initial radius are expected to be important governing parameters. An additional problem of interest is the interaction between a shockwave and a bubble at different phases in its cycle (i.e., out of equilibrium), where the optimal phase for maximum potential damage could be determined. Bubble collapse near different fluid media can be simulated. In addition, traveling cavitation can be considered, in which a bubble in a stagnation point flow travels towards the wall. These problems can be simulated by using the present code and simply changing the initial conditions.

Extending the code to three dimensions would allow the configuration of the problem to be changed. For instance, the direction of propagation of the shockwave could be changed, and multiple bubbles in a wide range of configurations could be considered, while more complex geometries could be implemented. More general 
traveling cavitation could be considered.

When considering bubble collapse near solid surface, the surface is assumed to be perfectly reflecting. In reality, there are coupled interactions between the solid body and the fluid. This coupling may be taken into account on a simple level by implementing an Immersed Boundary Method and prescribing appropriate conditions for the solid. A more refined solid mechanics method can be coupled to the present scheme to solve the full problem, and fracture mechanics may be implemented. However, adequate and efficient communication between the two simulations is challenging. With such models, bubble collapse near different types of solid media can be simulated, which are situations that occur often in bioengineering applications.

The present numerical method is not restricted to solving problems involving bubbles. Indeed, general compressible flows with interfaces may be computed. This includes the interaction of a droplet with high-speed gas flows or shockwaves, explosions near the ocean surface, the impact of liquid jets onto solid structures, breaking ocean waves, shock propagation in non-uniform media, or the impact of tidal waves on structures. 


\section{Appendix A}

\section{The stiffened equation of state}

In this appendix, additional relationships are derived for the stiffened equation of state. Certain thermodynamic quantities are required for the numerical scheme, and shock relations are necessary to set up appropriate initial conditions.

\section{A.1 Definitions}

\section{A.1.1 Isentropic flow}

The stiffened equation of state (Harlow \& Amsden, 1971) is given by

$$
\frac{1}{\gamma-1} p+\frac{\gamma P_{\infty}}{\gamma-1}=E-\rho \frac{u^{2}}{2}
$$

For perfect gases, $\gamma$ is the ratio of specific heats and $P_{\infty}=0$; for water, Section A.2.1 presents derivations for $\gamma$ and $P_{\infty}$. Starting with the Gibbs equation,

$$
d e=T d s-p d v=T d s+\frac{p}{\rho^{2}} d \rho
$$

where, $s$ is the entropy, $T$ is the temperature and $v$ is the specific volume, the internal energy $e=e(\rho, p)$ can be expressed:

$$
d e=\left.\frac{\partial e}{\partial p}\right|_{\rho} d p+\left.\frac{\partial e}{\partial \rho}\right|_{p} d \rho=\frac{1}{\gamma-1} \frac{d p}{\rho}-\frac{p+\gamma P_{\infty}}{\gamma-1} \frac{d \rho}{\rho^{2}} .
$$


For isentropic conditions, $d s=0$, so that

$$
\left.\frac{\partial e}{\partial \rho}\right|_{s}=\frac{p}{\rho^{2}}
$$

In a region where $\gamma$ and $P_{\infty}$ are constant (i.e., polytropic gas, where $\gamma \neq \gamma(T)$ ), Equation A.3 can be reorganized using Equation A.4, so that, along an isentrope,

$$
\frac{d\left(p+P_{\infty}\right)}{p+P_{\infty}}=\gamma \frac{d \rho}{\rho} \Rightarrow \frac{p+P_{\infty}}{P_{\infty}}=\left(\frac{\rho}{\rho_{o}}\right)^{\gamma}
$$

The latter equation is in a form similar to the Tait equation of state for water, where $\gamma=7.15$ and $P_{\infty}=3000$ atm (Thompson, 1984).

\section{A.1.2 Thermodynamic quantities}

Since $p=p(e, \rho)$,

$$
d p=\left.\frac{\partial p}{\partial e}\right|_{\rho} d e+\left.\frac{\partial p}{\partial \rho}\right|_{e} d \rho
$$

Using Equation A.4, the sound speed squared can be expressed

$$
c^{2}=\left.\frac{\partial p}{\partial \rho}\right|_{s}=\left.\frac{p}{\rho^{2}} \frac{\partial p}{\partial e}\right|_{\rho}+\left.\frac{\partial p}{\partial \rho}\right|_{e} .
$$

For the stiffened equation of state with constant $\gamma$ and $P_{\infty}$,

$$
\left.\frac{\partial p}{\partial e}\right|_{\rho}=(\gamma-1) \rho,\left.\quad \frac{\partial p}{\partial \rho}\right|_{e}=(\gamma-1) e
$$

Therefore,

$$
c^{2}=\gamma \frac{p+P_{\infty}}{\rho} .
$$

The enthalpy, $h=(E+p) / \rho$, using 2.6 and A.9, is given by:

$$
h=\frac{c^{2}}{\gamma-1}+\frac{u^{2}}{2}
$$


From Equation A.5, the specific volume can be written as:

$$
v=\frac{\alpha^{1 / \gamma}}{\left(p+P_{\infty}\right)^{1 / \gamma}}
$$

where $\alpha$ is some constant. Therefore,

$$
\begin{aligned}
& \left.\frac{\partial v}{\partial p}\right|_{s}=\frac{-\alpha^{\frac{1}{\gamma}}}{\gamma}\left(p+P_{\infty}\right)^{-\frac{\gamma+1}{\gamma}} \\
& \left.\frac{\partial^{2} v}{\partial p^{2}}\right|_{s}=\frac{\alpha^{\frac{1}{\gamma}}}{\gamma}\left(\frac{1}{\gamma}+1\right) \frac{1}{\left(p+P_{\infty}\right)^{\frac{2 \gamma-1}{\gamma}}}=\frac{\gamma+1}{\gamma^{2}} \frac{\left(p+P_{\infty}\right)^{-2}}{\rho}
\end{aligned}
$$

Using these results and Equation A.9, the fundamental gasdynamic derivative is given by:

$$
\Gamma=\left.\frac{\rho^{3} c^{4}}{2} \frac{\partial^{2} v}{\partial p^{2}}\right|_{s}=\frac{\rho^{3} c^{4}}{2} \frac{\gamma+1}{\gamma^{2}} \frac{1}{\rho\left(p+P_{\infty}\right)^{2}}=\frac{\rho^{3} c^{4}}{2} \frac{\gamma+1}{\gamma^{2}} \frac{1}{\rho\left(\rho c^{2} / \gamma\right)^{2}}=\frac{\gamma+1}{2}
$$

\section{A.1.3 Riemann invariants}

From the primitive form of the Euler equations 2.1 and the definition of the speed of sound, the following characteristic equations are obtained:

$$
d u \pm \frac{c}{\rho} d \rho=0, \quad \text { along } \frac{d x}{d t}=u \pm c .
$$

Using the definition of the speed of sound A.9 and the isentropic relation A.5,

$$
\int \frac{c}{\rho} d \rho=\sqrt{\gamma \frac{p+P_{\infty}}{\rho^{\gamma}}} \int \rho^{\frac{\gamma-3}{2}} d \rho=\sqrt{\gamma \frac{p+P_{\infty}}{\rho^{\gamma-1} \rho}} \frac{1}{\gamma-1} \rho^{\frac{\gamma-1}{2}}=\frac{2}{\gamma-1} c .
$$

Thus, Equation A.14 becomes:

$$
u \pm \frac{2}{\gamma-1} c=\text { const } \quad \text { along } \frac{d x}{d t}=u \pm c
$$




\section{A.2 Rankine-Hugoniot relations}

A shock traveling at speed, $s$, with constant $\gamma$ and $P_{\infty}$, is considered, in the frame of reference of the "unshocked" fluid. The goal is to express all the properties of the "shocked" fluid in terms of the properties of the "unshocked" fluid and the pressure jump across the shock.

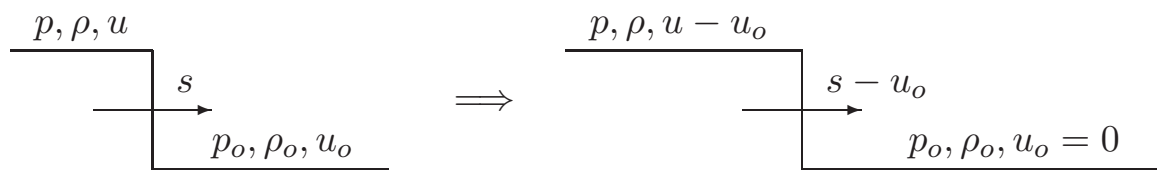

Figure A.1: Schematic of the reference frames for the shock.

The shock relations can be written for the Euler equations using a traveling wave analysis, where the brackets represent a jump in properties:

$$
\begin{aligned}
& -\left(s-u_{o}\right)[\rho]+[\rho u]=0, \\
& -\left(s-u_{o}\right)[\rho u]+\left[\rho u^{2}+p\right]=0, \\
& -\left(s-u_{o}\right)\left[\rho e+\rho \frac{u^{2}}{2}\right]+\left[u\left(\rho e+\rho \frac{u^{2}}{2}+p\right)\right]=0,
\end{aligned}
$$

Equation A.17a can be rewritten

$$
\begin{aligned}
\rho(s-u) & =\rho_{o}\left(s-u_{o}\right), \\
\frac{\rho_{o}}{\rho} & =1-\frac{u-u_{o}}{s-u_{o}},
\end{aligned}
$$

Rearranging A.17b:

$$
\begin{aligned}
p-p_{o} & =\rho_{o}\left(s-u_{o}\right)\left(u-u_{o}\right), \\
\left(s-u_{o}\right)^{2} & =\frac{p-p_{o}}{\rho_{o}\left(1-\frac{\rho_{o}}{\rho}\right)}
\end{aligned}
$$

where Equation A.18a was used for A.19a and Equation A.18b was used for Equa- 
tion A.19b. Equation A.17c can be rearranged:

$$
\begin{aligned}
\left(e+\frac{u^{2}}{2}\right) \rho(s-u) & =\left(e_{o}+\frac{u_{o}^{2}}{2}\right) \rho_{o}\left(s-u_{o}\right)-u_{o} p_{o} \\
e-e_{o} & =\frac{\left(u-u_{o}\right)^{2}}{2}-\left(\frac{\rho_{o}}{\rho}-1\right) \frac{p_{o}}{\rho_{o}}
\end{aligned}
$$

where Equations A.18a and A.19a) were used for Equation A.20b. Thus, using Equations A.18b and A.19b, Equation A.20b can be written as:

$$
e-e_{o}=\frac{p+p_{o}}{2}\left(\frac{1}{\rho_{o}}-\frac{1}{\rho}\right)
$$

Then, using Equation A.21 and the equation of state 2.6:

$$
\frac{\rho}{\rho_{o}}=\frac{\frac{\gamma+1}{\gamma-1} \frac{p+P_{\infty}}{p_{o}+P_{\infty}}+1}{\frac{\gamma+1}{\gamma-1}+\frac{p+P_{\infty}}{p_{o}+P_{\infty}}}
$$

Equation A.19b can be used with the definition of the speed of sound A.9 and Equation A.22 to find the shock speed:

$$
s=u_{o}+c_{o} \sqrt{\frac{\gamma+1}{2 \gamma}\left(\frac{p}{p_{o}}-1\right) \frac{p_{o}}{p_{o}+P_{\infty}}+1} .
$$

Equation A.19a can be used with the definition of the speed of sound A.9 and Equation A.23 to find the velocity:

$$
u=u_{o}+\frac{c_{o}}{\gamma} \frac{\left(\frac{p}{p_{o}}-1\right) \frac{p_{o}}{p_{o}+P_{\infty}}}{\sqrt{\frac{\gamma+1}{2 \gamma}\left(\frac{p}{p_{o}}-1\right) \frac{p_{o}}{p_{o}+P_{\infty}}+1}}
$$

It follows from Equation A.23 that the shock Mach number is given by:

$$
M_{s}=\frac{s-u_{o}}{c_{o}}=\sqrt{\frac{\gamma+1}{2 \gamma}\left(\frac{p}{p_{o}}-1\right) \frac{p_{o}}{p_{o}+P_{\infty}}+1},
$$


and the shock strength (nondimensional pressure difference) by:

$$
\Pi=\frac{p-p_{o}}{\rho c_{o}^{2}}=\frac{1}{\gamma}\left(\frac{p}{p_{o}}-1\right) \frac{p_{o}}{p_{o}+P_{\infty}} .
$$

The pressure jump across the shock can be re-written in terms of the Mach number:

$$
\frac{p}{p_{o}}=1+\frac{2 \gamma}{\gamma+1}\left(M^{2}-1\right)\left(1+\frac{P_{\infty}}{p_{o}}\right)
$$

\section{A.2.1 Determination of $\gamma$ and $P_{\infty}$}

The Hugoniot data for shock speed vs. particle speed in water is almost linear, as shown in Figure A.2 (Cocchi et al., 1996). Thus, as an approximation, $u_{s} \cong$ $c_{o}+\alpha u_{p}$, where $c_{o}=1647 \mathrm{~m} / \mathrm{s}, \alpha=1.921$ (slope of the dotted line), and $u_{s}=s-u_{o}$, $u_{p}=u-u_{o}$. From the Rankine-Hugoniot relations A.18b, A.19a, and A.22:

$$
u_{s}=\sqrt{c_{o}^{2}+\left(\frac{\gamma+1}{4} u_{p}\right)^{2}}+\frac{\gamma+1}{4} u_{p} .
$$

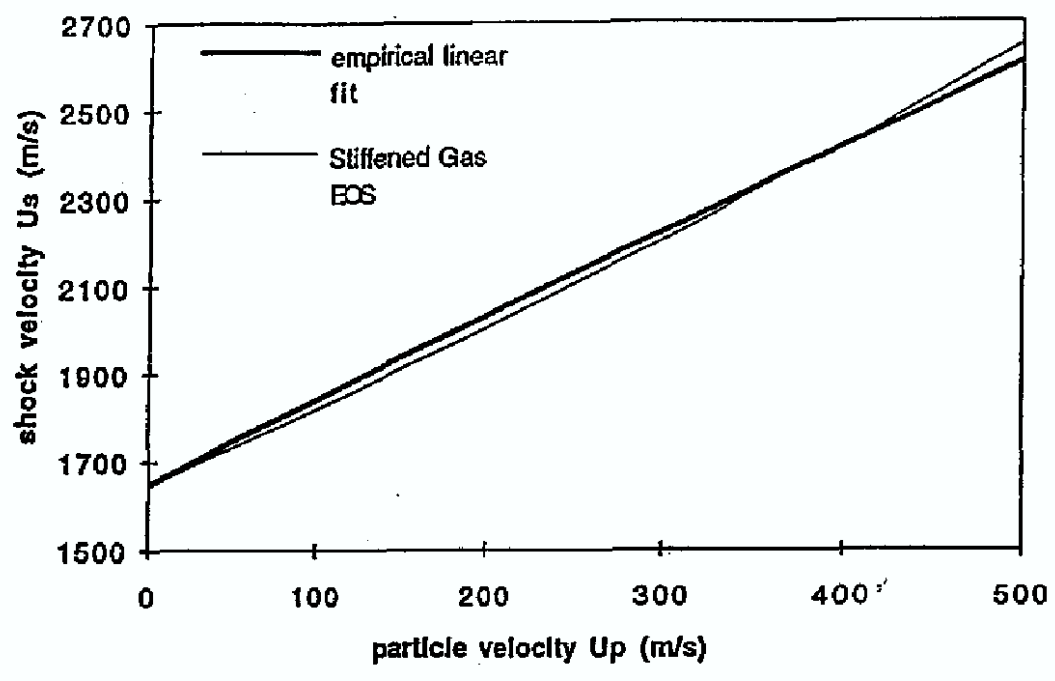

Figure A.2: Shock speed vs. particle speed in water. Reprinted with permission from Cocchi et al. (1996). 
Equation A.28 can then be rearranged to express $\gamma$ :

$$
\gamma=2 \alpha\left(1+\frac{1}{M_{s}}\right)-1
$$

Thus, given a shock Mach number (or $u_{s}$ ) and the slope, $\alpha$, from the experimental data, $\gamma$ can be determined. Then, $P_{\infty}$ is obtained by rearranging the equation of state $(2.6)$ :

$$
P_{\infty}=\frac{\rho_{o} c_{o}^{2}}{\gamma}-p_{o}
$$

where $p_{o}$ is taken to be the ambient pressure (atmospheric). Table A.2.1 lists values of $\gamma$ and $P_{\infty}$ that are used in the literature, along with the corresponding shock Mach number. For the present work, because the pressure ratios are such that the shock Mach number is close to unity, the values of $\gamma$ and $P_{\infty}$ are set as the limiting values as $M_{s} \rightarrow 1$. For the Tait equation, the corresponding shock Mach number would be less than unity, making the present analysis inconsistent.

Table A.1: Values of $\gamma, P_{\infty}$ used in the literature, with the corresponding shock Mach number.

\begin{tabular}{l|ccc} 
Reference & $M_{s}$ & $\gamma$ & $P_{\infty}$ \\
\hline Cocchi et al. (1996) & 1.445 & 5.50 & $4.92 \times 10^{8} \mathrm{~Pa}$ \\
Saurel \& Abgrall (1999b) & 2.466 & 4.40 & $6.15 \times 10^{8} \mathrm{~Pa}$ \\
Tait Eq. & 0.892 & 7.15 & $3.94 \times 10^{8} \mathrm{~Pa}$ \\
Present work & $1.005-1.050$ & 6.68 & $4.05 \times 10^{8} \mathrm{~Pa}$
\end{tabular}

\section{A.3 Expansion waves}

The isentropic relations derived from (A.5) can be used in an expansion fan. For example, given the pressure distribution,

$$
\frac{\rho}{\rho_{o}}=\left(\frac{p+P_{\infty}}{p_{o}+P_{\infty}}\right)^{1 / \gamma}, \quad \frac{c}{c_{o}}=\left(\frac{p+P_{\infty}}{p_{o}+P_{\infty}}\right)^{\frac{\gamma-1}{2 \gamma}}
$$


To determine the velocity, the fact that expansion waves are simple waves is used. Figure A.3 shows an $x t$-diagram for this case.

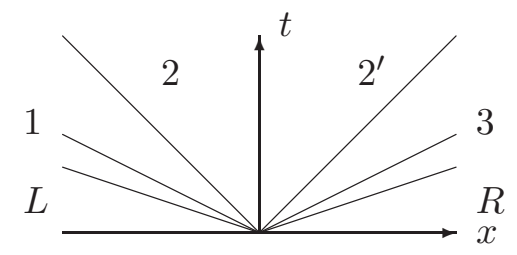

Figure A.3: Space-time diagram for expansion waves.

The points 2 and $2^{\prime}$ are equivalent. First, the left-moving expansion wave is considered. The $C^{+}$characteristics emanating from $L$ have uniform conditions. Therefore, in the entire left-hand plane,

$$
\frac{2}{\gamma-1} c+u=\frac{2}{\gamma-1} c_{L}+u_{L} \quad \Rightarrow \quad u_{2}=u_{L}+\frac{2}{\gamma-1}\left(1-\frac{c_{2}}{c_{L}}\right) c_{L}
$$

Along each of the fan $\left(C^{-}\right.$characteristics):

$$
u-\frac{2}{\gamma-1} c=\text { const, } \quad \text { along } \frac{d x}{d t}=u-c
$$

In view of equation (A.32), this can be rewritten as:

$$
u=\text { const }, \quad \text { along each } \frac{d x}{d t}=\frac{\gamma+1}{2} u-\frac{\gamma-1}{2} u_{L}-c_{L} .
$$

The value of $u$ will be different on each $C^{-}$, but the family of negative characteristics will be a family of straight lines, so that

$$
\frac{x}{t}=\frac{\gamma+1}{2} u_{1}-\frac{\gamma-1}{2} u_{L}-c_{L}
$$

The same holds for the right-moving expansion wave:

$$
u_{2}=u_{R}-\frac{2}{\gamma-1}\left(1-\frac{c_{2}}{c_{R}}\right) c_{R}, \quad \frac{x}{t}=\frac{\gamma+1}{2} u_{3}-\frac{\gamma-1}{2} u_{R}+c_{R} .
$$




\section{Appendix B}

\section{WENO reconstruction on non-uniform grids}

In the present section, the polynomials, ideal weights and smoothness indicators for fifth-order accurate WENO on a non-uniform grid are derived.

\section{B.1 WENO polynomials}

For WENO5 $(k=3)$, third-order accurate polynomials constitute the building blocks. For a non-uniform grid, Equation 3.18 can be modified to the following form (Shu, 1997):

$$
p^{(r)}(x)=\sum_{m=0}^{k} \sum_{j=0}^{m-1} \bar{v}_{i-r+j} \Delta x_{i-r+j} \frac{\sum_{\substack{l=0 \\ l \neq m}}^{k} \prod_{\substack{q=0 \\ q \neq m, l}}^{k}\left(x-x_{i-r+q-1 / 2}\right)}{\prod_{\substack{l=0 \\ l \neq m}}^{k}\left(x_{i-r+m-1 / 2}-x_{i-r+l-1 / 2}\right)},
$$

where $r$ denotes the left-most cell. Given the polynomials, the smoothness indicators are given by:

$$
\beta_{r}=\sum_{l=1}^{k-1} \int_{x_{i-1 / 2}}^{x_{i+1 / 2}}\left(x_{i+1 / 2}-x_{i-1 / 2}\right)^{2 l-1}\left(\frac{\partial^{l} p^{(r)}(\xi)}{\partial \xi^{l}}\right)^{2} d \xi
$$

The polynomials are combined to achieve fifth-order accuracy using ideal weights as follows:

$$
p_{i+1 / 2}=d_{0} p_{i+1 / 2}^{(0)}+d_{1} p_{i+1 / 2}^{(1)}+d_{2} p_{i+1 / 2}^{(2)}
$$


where the ideal weights have the following properties: $d_{r} \geq 0, \sum_{r} d_{r}=1$. A Mathematica worksheet was used to compute the polynomials, ideal weights, and smoothness indicators.

\section{B.2 Polynomial values at cell edges}

$$
\begin{aligned}
\boldsymbol{x} & =\boldsymbol{x}_{\boldsymbol{i + 1 / 2}} \\
r & =0: \\
& p_{i+1 / 2}^{(0)}=\bar{v}_{i}-\frac{\left(x_{i+3 / 2}-x_{i+1 / 2}\right)\left(x_{i+1 / 2}-x_{i-1 / 2}\right)}{\left(x_{i+5 / 2}-x_{i-1 / 2}\right)\left(x_{i+5 / 2}-x_{i+1 / 2}\right)}\left(\bar{v}_{i+2}-\bar{v}_{i+1}\right) \\
& \quad+\frac{\left(x_{i+1 / 2}-x_{i-1 / 2}\right)\left[\left(x_{i+3 / 2}-x_{i-1 / 2}\right)+\left(x_{i+5 / 2}-x_{i+1 / 2}\right)\right]}{\left(x_{i+3 / 2}-x_{i-1 / 2}\right)\left(x_{i+5 / 2}-x_{i-1 / 2}\right)}\left(\bar{v}_{i+1}-\bar{v}_{i}\right) .
\end{aligned}
$$

On a uniform grid, this simplifies to: $p_{i+1 / 2}^{(0)}=\left(-\frac{1}{6}\right) \bar{v}_{i+2}+\frac{5}{6} \bar{v}_{i+1}+\frac{2}{6} \bar{v}_{i}$. $r=1$ :

$$
\begin{aligned}
p_{i+1 / 2}^{(1)} & =\bar{v}_{i}+\frac{\left(x_{i+1 / 2}-x_{i-1 / 2}\right)\left(x_{i+1 / 2}-x_{i-3 / 2}\right)}{\left(x_{i+3 / 2}-x_{i-3 / 2}\right)\left(x_{i+3 / 2}-x_{i-1 / 2}\right)}\left(\bar{v}_{i+1}-\bar{v}_{i}\right) \\
& +\frac{\left(x_{i+1 / 2}-x_{i-1 / 2}\right)\left(x_{i+3 / 2}-x_{i+1 / 2}\right)}{\left(x_{i+1 / 2}-x_{i-3 / 2}\right)\left(x_{i+3 / 2}-x_{i-3 / 2}\right)}\left(\bar{v}_{i}-\bar{v}_{i-1}\right) .
\end{aligned}
$$

On a uniform grid, this simplifies to: $p_{i+1 / 2}^{(1)}=\frac{2}{6} \bar{v}_{i+1}+\frac{5}{6} \bar{v}_{i}+\left(-\frac{1}{6}\right) \bar{v}_{i-1}$. $r=2$ :

$$
\begin{aligned}
p_{i+1 / 2}^{(2)} & =\bar{v}_{i}+\left(\frac{x_{i+1 / 2}-x_{i-1 / 2}}{x_{i+1 / 2}-x_{i-5 / 2}}+\frac{x_{i+1 / 2}-x_{i-1 / 2}}{x_{i+1 / 2}-x_{i-3 / 2}}\right)\left(\bar{v}_{i}-\bar{v}_{i-1}\right) \\
& -\frac{\left(x_{i+1 / 2}-x_{i-1 / 2}\right)\left(x_{i+1 / 2}-x_{i-3 / 2}\right)}{\left(x_{i-1 / 2}-x_{i-5 / 2}\right)\left(x_{i+1 / 2}-x_{i-5 / 2}\right)}\left(\bar{v}_{i-1}-\bar{v}_{i-2}\right)
\end{aligned}
$$

On a uniform grid, this simplifies to: $p_{i+1 / 2}^{(2)}=\frac{11}{6} \bar{v}_{i}+\left(-\frac{7}{6}\right) \bar{v}_{i-1}+\frac{2}{6} \bar{v}_{i-2}$. 
$x=x_{i-1 / 2}$

$r=0$ :

$$
\begin{aligned}
p_{i-1 / 2}^{(0)} & =\bar{v}_{i}+\frac{\left(x_{i+1 / 2}-x_{i-1 / 2}\right)\left(x_{i+3 / 2}-x_{i-1 / 2}\right)}{\left(x_{i+5 / 2}-x_{i-1 / 2}\right)\left(x_{i+5 / 2}-x_{i+1 / 2}\right)}\left(\bar{v}_{i+2}-\bar{v}_{i+1}\right) \\
& -\left(\frac{x_{i+1 / 2}-x_{i-1 / 2}}{x_{i+3 / 2}-x_{i-1 / 2}}+\frac{x_{i+1 / 2}-x_{i-1 / 2}}{x_{i+5 / 2}-x_{i-1 / 2}}\right)\left(\bar{v}_{i+1}-\bar{v}_{i}\right) .
\end{aligned}
$$

On a uniform grid, this simplifies to: $p_{i-1 / 2}^{(0)}=\frac{2}{6} \bar{v}_{i+2}+\left(-\frac{7}{6}\right) \bar{v}_{i+1}+\frac{11}{6} \bar{v}_{i}$. $r=1$ :

$$
\begin{aligned}
p_{i-1 / 2}^{(1)} & =\bar{v}_{i}-\frac{\left(x_{i+1 / 2}-x_{i-1 / 2}\right)\left(x_{i-1 / 2}-x_{i-3 / 2}\right)}{\left(x_{i+3 / 2}-x_{i-3 / 2}\right)\left(x_{i+3 / 2}-x_{i-1 / 2}\right)}\left(\bar{v}_{i+1}-\bar{v}_{i}\right) \\
& -\frac{\left(x_{i+1 / 2}-x_{i-1 / 2}\right)\left(x_{i+3 / 2}-x_{i-1 / 2}\right)}{\left(x_{i+1 / 2}-x_{i-3 / 2}\right)\left(x_{i+3 / 2}-x_{i-3 / 2}\right)}\left(\bar{v}_{i}-\bar{v}_{i-1}\right) .
\end{aligned}
$$

On a uniform grid, this simplifies to: $p_{i-1 / 2}^{(1)}=\left(-\frac{1}{6}\right) \bar{v}_{i+1}+\frac{5}{6} \bar{v}_{i}+\frac{2}{6} \bar{v}_{i-1}$.

$r=2$ :

$$
\begin{aligned}
p_{i-1 / 2}^{(2)} & =\bar{v}_{i}-\frac{\left(x_{i+1 / 2}-x_{i-1 / 2}\right)\left[\left(x_{i+1 / 2}-x_{i-3 / 2}\right)+\left(x_{i-1 / 2}-x_{i-5 / 2}\right)\right]}{\left(x_{i+1 / 2}-x_{i-5 / 2}\right)\left(x_{i+1 / 2}-x_{i-3 / 2}\right)}\left(\bar{v}_{i}-\bar{v}_{i-1}\right) \\
& -\frac{\left(x_{i+1 / 2}-x_{i-1 / 2}\right)\left(x_{i-1 / 2}-x_{i-3 / 2}\right)}{\left(x_{i-1 / 2}-x_{i-5 / 2}\right)\left(x_{i+1 / 2}-x_{i-5 / 2}\right)}\left(\bar{v}_{i-1}-\bar{v}_{i-2}\right) .
\end{aligned}
$$

On a uniform grid, this simplifies to: $p_{i-1 / 2}^{(2)}=\frac{2}{6} \bar{v}_{i}+\frac{5}{6} \bar{v}_{i-1}+\left(-\frac{1}{6}\right) \bar{v}_{i-2}$.

\section{B.3 Ideal weights}

Only two weights are required because $d_{1}=1-d_{0}-d_{2}$.

$x=x_{i+1 / 2}$

$$
\begin{aligned}
& d_{0}=\frac{\left(x_{i+1 / 2}-x_{i-5 / 2}\right)\left(x_{i+1 / 2}-x_{i-3 / 2}\right)}{\left(x_{i+5 / 2}-x_{i-5 / 2}\right)\left(x_{i+5 / 2}-x_{i-3 / 2}\right)}, \\
& d_{2}=\frac{\left(x_{i+3 / 2}-x_{i+1 / 2}\right)\left(x_{i+5 / 2}-x_{i+1 / 2}\right)}{\left(x_{i+3 / 2}-x_{i-5 / 2}\right)\left(x_{i+5 / 2}-x_{i-5 / 2}\right)} .
\end{aligned}
$$


On a uniform grid, this simplifies to: $d_{0}=\frac{3}{10}, \quad d_{1}=\frac{6}{10}, \quad d_{2}=\frac{1}{10}$.

$x=x_{i-1 / 2}$

$$
\begin{aligned}
& d_{0}=\frac{\left(x_{i-1 / 2}-x_{i-5 / 2}\right)\left(x_{i-1 / 2}-x_{i-3 / 2}\right)}{\left(x_{i+5 / 2}-x_{i-5 / 2}\right)\left(x_{i+5 / 2}-x_{i-3 / 2}\right)}, \\
& d_{2}=\frac{\left(x_{i+3 / 2}-x_{i-1 / 2}\right)\left(x_{i+5 / 2}-x_{i-1 / 2}\right)}{\left(x_{i+3 / 2}-x_{i-5 / 2}\right)\left(x_{i+5 / 2}-x_{i-5 / 2}\right)} .
\end{aligned}
$$

On a uniform grid, this simplifies to: $d_{0}=\frac{1}{10}, \quad d_{1}=\frac{6}{10}, \quad d_{2}=\frac{3}{10}$.

\section{B.4 Smoothness indicators}

As Equation B.2 shows, the smoothness indicators have a unique value within a computational cell, regardless of where they are evaluated.

$$
\begin{aligned}
r & =0: \\
\beta_{0} & =\frac{4}{9} \frac{\left(x_{i+1 / 2}-x_{i-1 / 2}\right)}{\left(x_{i+3 / 2}-x_{i-1 / 2}\right)^{2}\left(x_{i+5 / 2}-x_{i-1 / 2}\right)^{2}\left(x_{i+5 / 2}-x_{i+1 / 2}\right)^{2}} \\
& \times\left\{81\left(x_{i+1 / 2}-x_{i-1 / 2}\right)^{3}\left[\left(\bar{v}_{i+2}-\bar{v}_{i+1}\right)\left(x_{i+3 / 2}-x_{i-1 / 2}\right)-\left(\bar{v}_{i+1}-\bar{v}_{i}\right)\left(x_{i+5 / 2}-x_{i+1 / 2}\right)\right]^{2}\right. \\
& +\left[\left\{-\left(x_{i+3 / 2}-x_{i+1 / 2}\right)\left[\left(\bar{v}_{i+2}-\bar{v}_{i+1}\right)\left(x_{i+3 / 2}-x_{i-1 / 2}\right)-\left(\bar{v}_{i+1}-\bar{v}_{i}\right)\left(x_{i+5 / 2}-x_{i+1 / 2}\right)\right]\right.\right. \\
& \left.+\left(\bar{v}_{i+2}-\bar{v}_{i+1}\right)\left(x_{i+3 / 2}-x_{i-1 / 2}\right)\left(x_{i+1 / 2}-x_{i-1 / 2}\right)+\left(\bar{v}_{i+1}-\bar{v}_{i}\right)\left(x_{i+5 / 2}-x_{i+1 / 2}\right)^{2}\right\}^{3} \\
& -\left\{\left[\left(x_{i+1 / 2}-x_{i-1 / 2}\right)+\left(x_{i+3 / 2}-x_{i-1 / 2}\right)\right]\right. \\
& \times\left[\left(\bar{v}_{i+2}-\bar{v}_{i+1}\right)\left(x_{i+3 / 2}-x_{i-1 / 2}\right)-\left(\bar{v}_{i+1}-\bar{v}_{i}\right)\left(x_{i+5 / 2}-x_{i+1 / 2}\right)\right] \\
& \left.\left.-\left(\bar{v}_{i+1}-\bar{v}_{i}\right)\left(x_{i+5 / 2}-x_{i+1 / 2}\right)\left(x_{i+5 / 2}-x_{i-1 / 2}\right)\right\}^{3}\right] \\
& \left.\times \frac{1}{\left(\bar{v}_{i+2}-\bar{v}_{i+1}\right)\left(x_{i+3 / 2}-x_{i-1 / 2}\right)-\left(\bar{v}_{i+1}-\bar{v}_{i}\right)\left(x_{i+5 / 2}-x_{i+1 / 2}\right)}\right\} .
\end{aligned}
$$

On a uniform grid, this simplifies to

$$
\beta_{0}=\frac{13}{12}\left[\left(\bar{v}_{i+2}-\bar{v}_{i+1}\right)-\left(\bar{v}_{i+1}-\bar{v}_{i}\right)\right]^{2}+\frac{3}{12}\left[\left(\bar{v}_{i+2}-\bar{v}_{i+1}\right)-3\left(\bar{v}_{i+1}-\bar{v}_{i}\right)\right]^{2} .
$$




$$
\begin{aligned}
r= & 1: \\
\beta_{1} & =\frac{4}{9} \frac{\left(x_{i+1 / 2}-x_{i-1 / 2}\right)}{\left(x_{i+1 / 2}-x_{i-3 / 2}\right)^{2}\left(x_{i+3 / 2}-x_{i-3 / 2}\right)^{2}\left(x_{i+3 / 2}-x_{i-1 / 2}\right)^{2}} \\
& \times\left\{81\left(x_{i+1 / 2}-x_{i-1 / 2}\right)^{3}\left[\left(\bar{v}_{i+1}-\bar{v}_{i}\right)\left(x_{i+1 / 2}-x_{i-3 / 2}\right)-\left(\bar{v}_{i}-\bar{v}_{i-1}\right)\left(x_{i+3 / 2}-x_{i-1 / 2}\right)\right]^{2}\right. \\
& +\left[\left\{\left(x_{i+1 / 2}-x_{i-1 / 2}\right)\left[\left(\bar{v}_{i+1}-\bar{v}_{i}\right)\left(x_{i+1 / 2}-x_{i-3 / 2}\right)-\left(\bar{v}_{i}-\bar{v}_{i-1}\right)\left(x_{i+3 / 2}-x_{i-1 / 2}\right)\right]\right.\right. \\
& \left.+\left(\bar{v}_{i+1}-\bar{v}_{i}\right)\left(x_{i+1 / 2}-x_{i-3 / 2}\right)^{2}+\left(\bar{v}_{i}-\bar{v}_{i-1}\right)\left(x_{i+3 / 2}-x_{i-1 / 2}\right)^{2}\right\}^{3} \\
& -\left\{\left(x_{i+1 / 2}-x_{i-1 / 2}\right)\left[\left(\bar{v}_{i+1}-\bar{v}_{i}\right)\left(x_{i+1 / 2}-x_{i-3 / 2}\right)-\left(\bar{v}_{i}-\bar{v}_{i-1}\right)\left(x_{i+3 / 2}-x_{i-1 / 2}\right)\right]\right. \\
& \left.\left.-\left(\bar{v}_{i+1}-\bar{v}_{i}\right)\left(x_{i+1 / 2}-x_{i-3 / 2}\right)\left(x_{i-1 / 2}-x_{i-3 / 2}\right)-\left(\bar{v}_{i}-\bar{v}_{i-1}\right)\left(x_{i+3 / 2}-x_{i-1 / 2}\right)^{2}\right\}^{3}\right] \\
& \left.\times \frac{1}{\left(\bar{v}_{i+1}-\bar{v}_{i}\right)\left(x_{i+1 / 2}-x_{i-3 / 2}\right)-\left(\bar{v}_{i}-\bar{v}_{i-1}\right)\left(x_{i+3 / 2}-x_{i-1 / 2}\right)}\right\} .
\end{aligned}
$$

On a uniform grid, this simplifies to

$$
\beta_{1}=\frac{13}{12}\left[\left(\bar{v}_{i+1}-\bar{v}_{i}\right)-\left(\bar{v}_{i}-\bar{v}_{i-1}\right)\right]^{2}+\frac{3}{12}\left(\bar{v}_{i}-\bar{v}_{i+1}\right)^{2}
$$

$r=2$ :

$$
\begin{aligned}
\beta_{2} & =\frac{4}{9} \frac{\left(x_{i+1 / 2}-x_{i-1 / 2}\right)}{\left(x_{i-1 / 2}-x_{i-5 / 2}\right)^{2}\left(x_{i+1 / 2}-x_{i-5 / 2}\right)^{2}\left(x_{i+1 / 2}-x_{i-3 / 2}\right)^{2}} \\
& \times\left\{81\left(x_{i+1 / 2}-x_{i-1 / 2}\right)^{3}\left[\left(\bar{v}_{i}-\bar{v}_{i-1}\right)\left(x_{i-1 / 2}-x_{i-5 / 2}\right)-\left(\bar{v}_{i-1}-\bar{v}_{i-2}\right)\left(x_{i+1 / 2}-x_{i-3 / 2}\right)\right]^{2}\right. \\
& -\left[\left\{\left(x_{i-1 / 2}-x_{i-3 / 2}\right)\left[\left(\bar{v}_{i}-\bar{v}_{i-1}\right)\left(x_{i-1 / 2}-x_{i-5 / 2}\right)-\left(\bar{v}_{i-1}-\bar{v}_{i-2}\right)\left(x_{i+1 / 2}-x_{i-3 / 2}\right)\right]\right.\right. \\
& \left.+\left(\bar{v}_{i}-\bar{v}_{i-1}\right)\left(x_{i-1 / 2}-x_{i-5 / 2}\right)^{2}+\left(\bar{v}_{i-1}-\bar{v}_{i-2}\right)\left(x_{i+1 / 2}-x_{i-3 / 2}\right)\left(x_{i+1 / 2}-x_{i-1 / 2}\right)\right\}^{3} \\
& -\left\{\left[\left(x_{i+1 / 2}-x_{i-1 / 2}\right)+\left(x_{i+1 / 2}-x_{i-1 / 2}\right)\right]\right. \\
& \times\left[\left(\bar{v}_{i}-\bar{v}_{i-1}\right)\left(x_{i-1 / 2}-x_{i-5 / 2}\right)-\left(\bar{v}_{i-1}-\bar{v}_{i-2}\right)\left(x_{i+1 / 2}-x_{i-3 / 2}\right)\right] \\
& \left.\left.+\left(\bar{v}_{i}-\bar{v}_{i-1}\right)\left(x_{i-1 / 2}-x_{i-5 / 2}\right)\left(x_{i+1 / 2}-x_{i-5 / 2}\right)\right\}^{3}\right] \\
& \left.\times \frac{1}{\left(\bar{v}_{i}-\bar{v}_{i-1}\right)\left(x_{i-1 / 2}-x_{i-5 / 2}\right)-\left(\bar{v}_{i-1}-\bar{v}_{i-2}\right)\left(x_{i+1 / 2}-x_{i-3 / 2}\right)}\right\} .
\end{aligned}
$$


On a uniform grid, this simplifies to

$$
\beta_{2}=\frac{13}{12}\left[\left(\bar{v}_{i}-\bar{v}_{i-1}\right)-\left(\bar{v}_{i-1}-\bar{v}_{i-2}\right)\right]^{2}+\frac{3}{12}\left[3\left(\bar{v}_{i}-\bar{v}_{i-1}\right)-\left(\bar{v}_{i-1}-\bar{v}_{i-2}\right)\right]^{2} .
$$




\section{Appendix C}

\section{Post-processing}

In this section, the methodology to calculate the properties of the bubble and of the re-entrant jet is explained.

\section{C.1 Interface location}

Although the interface is smeared over a few grid points, its actual location is required to compute certain variables (e.g., jet properties). For this purpose, the location of the interface is taken to be given by $\xi_{\text {int }}(\gamma=1.42)$.

\section{C.2 Bubble properties}

\section{C.2.1 Bubble volume}

In order to calculate the bubble volume, the volume fraction of gas, $\xi$, is first computed based on the distribution of $\gamma$ from Equation 3.62:

$$
\xi_{i j}=\frac{\gamma_{G}}{\gamma_{i j}} \frac{\gamma_{i j}-\gamma_{L}}{\gamma_{G}-\gamma_{L}}
$$

Then, the bubble volume is computed by summing the volume of gas in each cell leading to an averaged radius:

$$
V_{B}=\sum_{i j} \xi_{i j} \Delta V_{i j} \quad \Rightarrow \quad R=\left(\frac{3}{4 \pi} V_{B}\right)^{1 / 3}
$$


In cylindrical coordinates,

$$
\Delta V_{i j}=2 \pi r_{j} \Delta r_{j} \Delta x_{i}
$$

The collapse time is taken to be the time at which the bubble volume is minimum.

\section{C.2.2 Bubble position}

The position of the bubble is computed by determining its center of "volume" along the $x$-axis (its radial position will always be zero). This is done by taking the average position of all the cells that contain mainly gas:

$$
H=\frac{\sum_{\xi_{i j} \leq \xi_{i n t}} \xi_{i}}{N_{\xi_{i j} \leq \xi_{i n t}}},
$$

where $N_{\xi_{i j} \leq \xi_{i n t}}$ is the number of cells for which $\xi<0.5$.

\section{C.2.3 Bubble pressure}

In Rayleigh-Plesset models, the pressure within the bubble is assumed to be uniform. However, results from the numerical simulations show clear variations within the bubble, especially due to the propagation of shockwaves. Thus, the bubble pressure is computed by taking the average value of the pressure within the bubble, as follows:

$$
p_{B}=\frac{\sum_{i j} p_{i j} \xi_{i j} \Delta V_{i j}}{V_{B}}
$$

\section{C.2.4 Bubble non-sphericity}

By considering the $x r$-plane passing through $r=0$, the length of the bubble outline can be computed. To do so, coordinates of the interface are recorded by interpolating the $x$-value for $r_{j}, \forall j$, and the $r$-value for $x_{i}, \forall i$. Starting with the first pair along $r=0$, these pairs are ordered by considering the distance with all the other points. Then, the length of the bubble is computed by adding the 
distance between each point (first order approximation):

$$
L_{B}=\sum_{i} \sqrt{\left(x_{i+1}-x_{i}\right)^{2}+\left(r_{i+1}-r_{i}\right)^{2}}
$$

Similarly, the area of the bubble can be computed by "integrated" the area within the bubble outline:

$$
A_{B}=\sum_{i}\left(r_{i+1}-r_{i}\right) \frac{x_{i+1}-x_{i}}{2} .
$$

The ratio,

$$
\nu=\frac{A_{B}}{\bar{R} L_{B}}
$$

provides a measure of non-sphericity of the bubble, where $\bar{R}$ is an average value for the bubble radius. For a spherical collapse, $\nu=1 / 2$; for a highly non-spherical $\nu \rightarrow 0$. The average radius is computed from the bubble area,

$$
\bar{R}=R_{o} \sqrt{\frac{A_{B}}{A_{B, o}}}=\sqrt{\frac{A}{\pi}} .
$$

Furthermore, taking the time-derivative of $\bar{R}$,

$$
\dot{R} \approx \dot{\bar{R}}=\frac{\bar{R}^{n+1}-\bar{R}^{n}}{\Delta t}
$$

allows the approximation of the speed of the bubble wall when computing quantities such as the radiated energy.

\section{C.3 Circulation}

The total circulation of the domain is computed by summing up the vorticity projected onto the area of each cell:

$$
\Gamma=\int_{A} \boldsymbol{\omega} \cdot d \mathbf{A}=\sum_{i j} \omega_{i j} \Delta x_{i} \Delta r_{j}
$$

Thus, the circulation is computed along an $x r$-plane that passes through $r=0$. 


\section{C.4 Jet properties}

The position, pressure, density, and sound speed of the jet (and of the distal side) are taken to be the local values of these variables at $\xi_{i, 0}=\xi_{\text {int }}$ by using linear interpolation:

$$
\eta_{j e t}=\eta_{i, 0}+\frac{\xi_{i n t}-\xi_{i, 0}}{\xi_{i+1,0}-\xi_{i, 0}}\left(\eta_{i+1,0}-\eta_{i, 0}\right)
$$

where $\eta$ is any of the aforementioned variables. The speed of the jet (and of the distal side) is computed by computing a first order derivative of the position:

$$
v_{j e t}=\frac{x_{j e t}^{(n)}-x_{j e t}^{(n-1)}}{\Delta t},
$$

where the superscript denotes the time level.

\section{C.5 Numerical Schlieren contours}

Plots of the density gradient are generated in order to emulate experimental Schlieren images, adapting the methodology of Quirk \& Karni (1996). This allows waves and interfaces to be visualized on the same plots. The following function of the density gradient is plotted:

$$
f_{i j}=\exp \left(-\alpha \frac{|\nabla \rho|}{\max |\nabla \rho|}\right),
$$

where $\alpha=40$ for air and $\alpha=400$ for water, in order to observe waves in both fluids simultaneously. 


\section{Bibliography}

AbGrall, R 1996 How to prevent pressure oscillations in multicomponent flow calculations: a quasi conservative approach. J. Comput. Phys. 125, 150-160.

Abgrall, R \& Karni, S 2001 Computations of compressible multifluids. J. Comput. Phys. 169, 594-623.

Abgrall, R \& Saurel, R 2003 Discrete equations for physical and numerical compressible multiphase mixtures. J. Comput. Phys. 186, 361-396.

Acosta, A J 2000 Cavitation and cavitation types. In Cavitation of Hydraulic Machinery (ed. S C Li). Imperial College Press.

ARndT, R E A 1981 Cavitation in fluid machinery and hydraulic structures. Annu. Rev. Fluid Mech. 12, 273-328.

Ball, G J, Howell, B P, Leighton, T G \& Schofield, M J 2000 Shockinduced collapse of a cylindrical air cavity in water: a Free-Lagrange simulation. Shock Waves 10, 265-276.

Balsara, D S \& Shu, C W 2000 Monotonicity preserving weighted essentially non-oscillatory schemes with increasingly high order of accuracy. J. Comput. Phys. 160, 405-452.

Batten, P, Clarke, N, Lambert, C \& Causon, D 1997 On the choice of wave speeds for the HLLC Riemann solver. SIAM J. Sci. Comput. 18, 1553-1570.

Benjamin, B 1958 Pressure waves from collapsing cavities. In Proc. Second ONR Symp. Naval Hydrodynamics, pp. 207-233. 
Benjamin, T B \& Ellis, A T 1966 The collapse of cavitation bubbles and the pressure thereby produced against solid boundaries. Phil. Trans. R. Soc. Lond. A 260, 221-240.

Besant, W H 1859 Hydrostatics and Hydrodynamics. Cambridge University Press.

Best, J P 1993 The formation of toroidal bubbles upon the collapse of transient cavities. J. Fluid Mech. 251, 79-107.

Best, J P \& KuCERA, A 1992 A numerical investigation of non-spherical rebounding bubbles. J. Fluid Mech. 245, 137-154.

Billet, G \& ABGRAll, R 2003 An adaptive shock-capturing algorithm for solving unsteady reactive flows. Comput. Fluids 32, 1473-1495.

Birkhoff, G 1954 Note on Taylor instability. Quart. Appl. Math. 13, 306-309.

Blake, J R, Taib, B B \& Doherty, G 1986 Transient cavities near boundaries. Part 1. Rigid boundary. J. Fluid Mech. 170, 479-497.

Bourne, N K \& Field, J E 1992 Shock-induced collapse of single cavities in liquids. J. Fluid Mech. 244, 225-240.

Brennen, C E 1994 Hydrodynamics of Pumps. Oxford University Press.

Brennen, C E 1995 Cavitation and Bubble Dynamics. Oxford University Press.

Brennen, C E 2002 Fission of collapsing cavitation bubbles. J. Fluid Mech. 472, $153-166$.

Busnaina, A A, Kashkoush, I I \& Gale, G W 1995 An experimental study of megasonic cleaning of silicon wafers. J. Electrochem. Soc. 142, 2812-2817.

Chang, C H \& Liou, M S 2007 A robust and accurate approach to computing compressible multiphase flow: Stratified flow model and $\mathrm{AUSM}^{+}$-up scheme. $J$. Comput. Phys. 225, 840-873. 
Chaussy, C 1982 Extracorporeal Shock Wave Lithotripsy: New Aspects in the Treatment of Kidney Stone Disease. Basel: Karger.

Chaussy, C 1986 Extracorporeal Shock Wave Lithotripsy: Technical Concept, Experimental Research, and Clinical Application. Basel: Karger.

Church, C C 1989 A theoretical study of cavitation generated by an extracorporeal shock wave lithotripter. J. Acoust. Soc. Am. 86, 215-227.

Cleveland, R O, Bailey, M R, Fineberg, N, Hartenbaum, B, Lokhandwalla, M, McAteer, J A \& Sturtevant, B 2000 Design and characterization of a research electrohydraulic lithotripter patterned after the Dornier HM3. Rev. Sci. Instrum. 71, 2514-2525.

Cleveland, R O \& Sapozhnikov, O A 2005 Modeling elastic wave propagation in kidney stones with application to shock wave lithotripsy. J. Acoust. Soc. Am. 118, 2667-2676.

Cocchi, J P, Saurel, R \& Loraud, J C 1996 Treatment of interface problems with Godunov-type schemes. Shock Waves 5, 347-357.

Coe, F L, Evan, A \& Worcester, E 2005 Kidney stone disease. J. Clin. Invest. 115, 2598-2608.

Cohen, N P \& Whitfield, H N 1993 Mechanical testing of urinary calculi. World J. Urol. 11, 1-18.

Cole, R 1948 Underwater Explosions. Princeton, NJ: Princeton University Press.

Coleman, A J \& Saunders, J E 1989 A survey of the acoustic output of commercial extracorporeal shock wave lithotripters. Ultrasound Med. Biol. 15, 213-227.

Coleman, A J, Saunders, J E, Crum, L A \& Dyson, M 1987 Acoustic cavitation generated by an extracorporeal shockwave lithotripter. Ultrasound Med. Biol. 13, 69-76. 
Colonius, T 2004 Modeling artificial boundary conditions for compressible flow. Annu. Rev. Fluid Mech. 36, 315-345.

Colonius, T \& RAN, H 2002 A super-grid-scale model for simulating compressible flow on unbounded domains. J. Comput. Phys. 182, 191-212.

Crum, L A 1980 Measurements of the growth of air bubbles by rectified diffusion. J. Acoust. Soc. Am. 68, 203-211.

Crum, L A 1988 Cavitation microjets as a contributory mechanism for renal calculi disintegration in ESWL. J. Urol. 140, 1587-1590.

DeAR, J P \& Field, J E 1988 A study of the collapse of arrays of cavities. J. Fluid Mech. 190, 409-425.

Delius, M 1994 Medical applications and bioeffects of extracorporeal shock waves. Shock Waves 4, 55-72.

Dowell, E H \& Hall, K C 2001 Modeling of fluid-structure interaction. Annu. Rev. Fluid Mech. 33, 445-490.

Einfeldt, B, Munz, C D, Roe, P L \& Sjögreen, B 1991 On Godunov-type methods near low densities. J. Comput. Phys. 92, 273-295.

EISENMENGER, W 2001 The mechanisms of stone fragmentation in ESWL. Ultrasound Med. Biol. 27, 683-693.

Elder, S A 1959 Cavitation microstreaming. J. Acoust. Soc. Am. 31, 54-64.

Evan, A P, Willis, L R, Lingeman, J E \& McAteer, J A 1998 Renal trauma and the risk of long-term complications in shock wave lithotripsy. Nephron $\mathbf{7 8}$, $1-8$.

Evan, A P, Willis, L R, McAteer, J A, Balley, M R, Connors, B A, Shao, Y Z, Lingeman, J E, Williams, J C, Fineberg, N S \& Crum, L A 2002 Kidney damage and renal functional changes are minimized by waveform 
control that suppresses cavitation in shock wave lithotripsy. J. Urol. 168, 15561562.

FAVre, A 1965 Équations des gaz turbulents compressibles. I. Formes générales. J. Mécanique 4, 361-390.

Fedkiw, R P, Aslam, T, Merriman, B \& Osher, S 1999 A non-oscillatory Eulerian approach to interfaces in multimaterial flows (the Ghost Fluid Method). J. Comput. Phys. 152, 457-492.

Fedkiw, R P, Merriman, B \& Osher, S 2000 Simplified discretization of systems of hyperbolic conservation laws containing advection equations. J. Comput. Phys. 157, 302-326.

Flannigan, D J, Hopkins, S D, Camara, C G, Putterman, S J \& Suslick, K S 2006 Measurement of pressure and density inside a single sonoluminescing bubble. Phys. Rev. Lett. 96, 204301.

Franc, J P \& Michel, J M 2004 Fundamentals of Cavitation. Kluwer Academic Publishers.

Fujikawa, S \& Akamatsu, T 1980 Effects of the non-equilibrium condensation of vapour on the pressure wave produced by the collapse of a bubble in a liquid. J. Fluid Mech. 97, 481-512.

Gilmore, F R 1952 The growth and collapse of a spherical bubble in a viscous compressible liquid. Tech. Rep. Rep. 26-4. Calif. Inst. Technol. Eng. Div.

Gottlieb, S \& Shu, C W 1998 Total variation diminishing Runge-Kutta schemes. Math. Comp. 67, 73-85.

Gracewski, S M, Dahake, G, Ding, Z, Burns, S J \& Everbach, E C 1993 Internal stress wave measurements in solids subjected to lithotripter pulses. $J$. Acoust. Soc. Am. 94, 652-661. 
Grasso, F \& Pirozzoli, S 2000 Shock wave - thermal inhomogeneity interactions: Analysis and numerical simulations of sound generation. Phys. Fluids 12, 205-219.

HaAs, J F \& Sturtevant, B 1987 Interaction of weak shock waves with cylindrical and spherical gas inhomogeneities. J. Fluid Mech. 181, 41-76.

Haines, J R, Riemer, B W, Felde, D K, Hunn, J D, Pawel, S J \& Tai, C C 2005 Summary of cavitation erosion investigations for the SNS mercury target. J. Nucl. Mater. 343, 58-69.

Hammitt, F G 1980 Cavitation and Multiphase Flow Phenomena. McGraw-Hill.

Hansson, I, Kedrinskit, V \& Mørch, K A 1982 On the dynamics of cavity clusters. J. Phys. D: Appl. Phys. 12, 1725-1734.

Harlow, F \& Amsden, A 1971 Fluid dynamics. Tech. Rep. LANL Monograph LA-4700. Los Alamos National Labs.

Harten, A, Engquist, B, Osher, S \& Chakravarthy, S 1987 Uniformly high order accurate essentially nonoscillatory schemes, III. J. Comput. Phys. 71, 231-303.

Harten, A, Lax, P D \& van Leer, B 1983 On upstream differencing and Godunov-type schemes for hyperbolic conseration laws. SIAM Review 25, 3561.

Henderson, L F 1989 On the refraction of shock waves. J. Fluid Mech. 198, $365-386$.

Henrick, A K, Aslam, T D \& Powers, J M 2005 Mapped weighted essentially non-oscillatory schemes: achieving optimal order near critical points. J. Comput. Phys. 207, 542-567. 
Herring, C 1941 Theory of the pulsations of the gas bubble produced by an underwater explosion. Tech. Rep. NDRC Division 6 Report C4-sr20. National Defense Research Committee.

Hickling, R 1963 Effects of thermal conduction in sonoluminescence. J. Acoust. Soc. Am. 35, 967-974.

Hickling, R \& Plesset, M S 1964 Collapse and rebound of a spherical bubble in water. Phys. Fluids 7, 7-14.

Holt, M 1977 Underwater explosions. Annu. Rev. Fluid Mech. 9, 187-214.

Hsieh, D Y \& Plesset, M S 1961 Theory of rectified diffusion of mass into gas bubbles. J. Acoust. Soc. Am. 33, 206-215.

Hu, X Y, Khoo, B C, Adams, N A \& Huang, F L 2006 A conservative interface method for compressible flows. J. Comput. Phys. 219, 553-578.

Huang, Y C, Hammitt, F G \& Mitchell, T M 1973 Note on shock-wave velocity in high-speed liquid-solid impact. J. Appl. Phys. 44, 1868-1869.

JAmALudDin, A R 2005 Free-lagrange simulations of shock-bubble interaction in extracorporeal shock wave lithotripsy. PhD thesis, University of Southampton.

Jenny, P, Müller, B \& Thomann, H 1997 Correction of conservative Euler solvers for gas mixtures. J. Comput. Phys. 132, 91-107.

JiANG, G S \& SHU, C W 1996 Efficient implementation of weighted ENO schemes. J. Comput. Phys. 126, 202-228.

Johnsen, E \& Colonius, T 2006 Implementation of WENO schemes for compressible multicomponent flow problems. J. Comput. Phys. 219, 715-732.

JosePh, D D 1989 Fluid dynamics of viscoelastic liquids. Springer-Verlag.

Joseph, D D, Belanger, J \& Beavers, G S 1999 Breakup of a liquid drop suddenly exposed to a high-speed airstream. Int. J. Multiphase Flow 25, 12631303. 
Kameda, M \& Matsumoto, Y 1996 Shock waves in a liquid containing small gas bubbles. Phys. Fluids 8, 322-335.

Kameda, M, Shimaura, N, Higashino, F \& Matsumoto, Y 1998 Shock waves in a uniform bubbly flow. Phys. Fluids 10, 2661-2668.

KARNI, S 1994 Multicomponent flow calculations by a consistent primitive algorithm. J. Comput. Phys. 112, 31-43.

KARni, S 1996 Hybrid multifluid algorithms. SIAM J. Sci. Comput. 17, 10191039.

Klaseboer, E, Turangan, C, Fong, S W, Liu, T G, Hung, K C \& Khoo, B C 2006 Simulations of pressure pulse-bubble interaction using boundary element method. Comput. Methods Appl. Mech. Engrg. 195, 4287-4302.

Kling, C L \& Hammitt, F G 1972 A photographic study of spark-induced cavitation bubble collapse. Trans. ASME D: J. Basic Eng. 94, 825-833.

KNAPP, R T 1952 Cavitation mechanics and its relation to the design of hydraulic equipment. Proc. Inst. Mech. Eng. (A) 166, 150-163.

Knapp, R T, Daily, J W \& Hammitt, F G 1970 Cavitation. McGraw-Hill.

Koren, B, Lewis, M R, van Brummelen, E H \& van Leer, B 2002 Riemannproblem and level-set approaches for homentropic two-fluid flow computations. J. Comput. Phys. 181, 654-674.

Kornfeld, M \& Suvorov, L 1944 On the destructive action of cavitation. J. Appl. Phys. 15, 495-506.

Koumoutsakos, P 2005 Multiscale flow simulations using particles. Annu. Rev. Fluid Mech. 37, 457-487.

Larrouturou, B \& Fezoui, L 1989 On the equations of multi-component perfect or real gas inviscid flow. In Lecture Notes in Mathematics: Nonlinear Hy- 
perbolic Systems (ed. C Carasso, P Charrier, B Hanouzet \& J L Joly). SpringerVerlag.

LAUterborn, W \& Bolle, H 1975 Experimental investigations of cavitationbubble collapse in the neighbourhood of a solid boundary. J. Fluid Mech. 72, 391-399.

LAX, P 1954 Weak solutions of nonlinear hyperbolic equations and their numerical computation. Comm. Pure Appl. Math. 7, 159-193.

LeVeque, R J 1992 Numerical Methods for Conservation Laws. BirkhauserVerlag.

LeVeque, R J 2002 Finite Volume Methods for Hyperbolic Problems. Cambridge University Press.

LI, S C 2000 Introduction. In Cavitation of Hydraulic Machinery (ed. S C Li). Imperial College Press.

Lindau, O \& LAUterborn, W 2003 Cinematographic observation of the collapse and rebound of a laser-produced cavitation bubble near a wall. J. Fluid Mech. 479, 327-348.

Liu, T G, Khoo, B C \& Yeo, K S 2003 Ghost fluid method for strong shock impacting on material interface. J. Comput. Phys. 190, 651-681.

LiU, X D, Osher, S \& Chan, T 1994 Weighted essentially non-oscillatory schemes. J. Comput. Phys. 115, 200-212.

Lokhandwalla, M \& Sturtevant, B 2000 Fracture mechanics model of stone comminution in ESWL and implications for tissue damage. Phys. Med. Biol. 45, 1923-1940.

Lokhandwalla, M \& Sturtevant, B 2001 Mechanical haemolysis in shock wave lithotripsy (swl): I. Analysis of cell deformation due to swl flow-fields. Phys. Med. Biol. 46, 413-437. 
Lubock, P \& Goldsmith, W 1980 Experimental cavitation studies in a model head-neck system. J. Biomech. 13, 1041-1052.

Marquina, A \& Mulet, P 2003 A flux-split algorithm applied to conservative models for multicomponent compressible flows. J. Comput. Phys. 185, 120-138.

McNamara III, W B, Didenko, Y T \& Suslick, K S 1999 Sonoluminescence temperatures during multi-bubble cavitation. Nature 401, 772-775.

Menikoff, R \& Plohr, B J 1989 The Riemann problem for fluid flow of real materials. Rev. Mod. Phys. 61, 75-130.

Moholkar, V S, Warmoeskerken, M M C G, Ohl, C D \& Prosperetti, A 2004 Mechanism of mass-transfer enhancement in textiles by ultrasound. AIChE J. 60, 58-64.

Mourad, P D, Roberts, F A \& McInnes, C 2007 Synergistic use of ultrasound and sonic motion for removal of dental plaque bacteria. Compend. Contin. Educ. Dent. 28, 354-358.

Mulder, W, Osher, S \& Sethian, J A 1992 Computing interface motion in compressible gas dynamics. J. Comput. Phys. 100, 209-228.

Nagrath, S, Jansen, K, Lahey JR., R T \& Akhatov, I 2006 Hydrodynamic simulation of air bubble implosion using a level set approach. J. Comput. Phys. 215, 98-132.

NAudé, C F \& Ellis, A T 1961 On the mechanism of cavitation damage by nonhemisperical cavities in contact with a solid boundary. Trans. ASME D: J. Basic Eng. 83, 648-656.

Niederhaus, J H J, Greenough, J A, Oakley, J G, Ranjan, D, Anderson, M H \& BonazzA, R 2007 A computational parameter study for the threedimensional shock-bubble interaction. In press. 
NIH 2004 Kidney stones in adults. On the WWW, uRL http://kidney.niddk.nih.gov.

Nourgaliev, R R, Dinh, $\mathrm{T}$ N \& Theofanous, T G 2006 Adaptive characteristics-based matching for compressible multifluid dynamics. J. Comput. Phys. 213, 500-529.

Ohl, C D, Arora, M, Dijkink, R, Janve, V \& Lohse, D 2006 Surface cleaning from laser-induced cavitation bubbles. Appl. Phys. Lett. 89, 074102.

OHL, C D \& IKINK, R 2003 Shock-wave-induced jetting of micron-size bubbles. Phys. Rev. Lett. 90, 1-4.

Ohl, C D, Kurz, T, Geisler, R, Lindau, O \& Lauterborn, W 1999 Bubble dynamics, shock waves and sonoluminescence. Phil. Trans. R. Soc. Lond. A 357, 269-294.

Osher, S \& Sethian, J A 1988 Fronts propagating with curvature-dependent speed: algorithms based on Hamilton-Jacobi formulations. J. Comput. Phys. 79, $12-49$.

Packer, M, Fishkind, W J, Fine, I H, Seibel, B S \& Hoffman, R S 2005 The physics of phaco: a review. J. Cataract Refract. Surg. 31, 424-431.

Paterson, R F, Lifshitz, D A, Lingeman, J E, Evan, A P, Connors, B A, Fineberg, N S, Williams, J C \& McAteer, J A 2002 Stone fragmentation during shock wave lithotripsy is improved by slowing the shock wave rate: Studies with a new animal model. J. Urol. 168, 2211-2215.

Peng, G, Zabusky, N J \& Zhang, S 2003 Vortex-accelerated secondary baroclinic vorticity deposition and late-intermediate time dynamics of a twodimensional Richtmyer-Meshkov interface. Phys. Fluids 15, 3730-3744.

Philipp, A, Delius, M, Scheffczyk, C, Vogel, A \& Lauterborn, W 1993 Interaction of lithotripter-generated shock waves with air bubbles. J. Acoust. Soc. Am. 93, 2496-2509. 
Philipp, A \& Lauterborn, W 1998 Cavitation erosion by single laser-produced bubbles. J. Fluid Mech. 361, 75-116.

PiCONe, J M \& BorIs, J P 1988 Vorticity generation by shock propagation through bubbles in a gas. J. Fluid Mech. 189, 23-51.

Pishchalnikov, Y A, Sapozhnikov, O A, Bailey, M R, Williams, J C, Cleveland, R O, Colonius, T, Crum, L A, Evan, A P \& McAteer, J A 2003 Cavitation bubble cluster activity in the breakage of kidney stones by lithotripter shockwaves. J. Endourol. 17, 435-446.

Plesset, M S 1949 The dynamics of cavitation bubbles. Trans. ASME: J. Appl. Mech. 16, 277-282.

Plesset, M S 1969 Cavitating flows. In Topics in Ocean Engineering (ed. C I Bretschneider). Gulf Publ. Co.

Plesset, M S \& Chapman, R B 1971 Collapse of an initially spherical vapour cavity in the neighbourhood of a solid boundary. J. Fluid Mech. 47, 283-290.

Plesset, M S \& Mitchell, T P 1956 On the stability of the spherical shape of a vapor cavity in a liquid. Quart. Appl. Math. 13, 419-430.

Plesset, M S \& Prosperetti, A 1977 Bubble dynamics and cavitation. Annu. Rev. Fluid Mech. 9, 145-185.

Plesset, M S \& Zwick, S A 1954 A nonsteady heat diffusion problem with spherical symmetry. J. Appl. Phys. 23, 95-98.

Popinet, S \& ZALESKi, S 2002 Bubble collapse near a solid boundary: a numerical study of the influence of viscosity. J. Fluid Mech. 464, 137-163.

POWER, H 1995 Boundary element applications in fluid mechanics. WIT Press.

Putterman, S J \& Weninger, K R 2000 Sonoluminescence: how bubbles turn sound into light. Annu. Rev. Fluid Mech. 32, 445-476. 
QIU, J \& SHU, C W 2002 On the construction, comparison, and local characteristic decomposition for high-order central WENO schemes. J. Comput. Phys. 183, 187-209.

QuiRK, J J \& KARNI, S 1996 On the dynamics of a shock-bubble interaction. J. Fluid Mech. 318, 129-163.

Rattray, M 1951 Perturbation effects in cavitation bubble dynamics. PhD thesis, California Institute of Technology.

RAYLEIGH, LORD 1917 On the pressure developed in a liquid during the collapse of a spherical cavity. Phil. Mag. 34, 94-98.

Riesch, E S 2007 Purdue dogged by misconduct claims. Nature 447, 238-239.

RoE, P L 1981 Approximate Riemann solvers, parameter vectors, and difference schemes. J. Comput. Phys. 43, 357-372.

Samtaney, R \& Pullin, D I 1996 On initial-value and self-similar solutions of the compressible Euler equations. Phys. Fluids 8, 2650-2655.

Sankin, G N, Simmons, W N, Zhu, S L \& Zhong, P 2005 Shock wave interaction with laser-generated single bubbles. Phys. Rev. Lett. 95, 034501.

Sapozhnikov, O A, Khokhlova, V A, Bailey, M R, Williams, J C, McAteer, J A, Cleveland, R O \& Crum, L A 2002 Effect of overpressure and pulse repetition frequency on cavitation in shock wave lithotripsy. J. Acoust. Soc. Am. 112, 1183-1195.

SAURel, R \& ABGRALL, R 1999 a A multiphase Godunov method for compressible multifluid and multiphase flows. J. Comput. Phys. 150, 425-467.

SAurel, R \& AbGrall, R $1999 b$ A simple method for compressible multifluid flows. SIAM J. Sci. Comput. 21, 1115-1145.

Sethian, J A 1996 Level Set Methods. Cambridge University Press. 
Shima, A, Tomita, Y \& Takahashi, K 1984 The collapse of a gas bubble near a solid wall by a shock wave and the induced impulsive pressure. Proc. Inst. Mech. Eng. 198C, 81-86.

SHU, C W 1988 Total-variation-diminishing time discretizations. SIAM J. Sci. Stat. Comput. 9, 1073-1084.

SHu, C W 1997 Essentially non-oscillatory and weighted essentially non-oscillatory schemes for hyperbolic conservation laws. Tech. Rep. ICASE Report No. 97-65. NASA Langley Research Center.

SHu, C W 1999 High order ENO and WENO schemes for computational fluid dynamics. In High-Order Methods for Computational Physics (ed. T J Barth \& H Deconinck). Springer-Verlag.

SHu, C W 2003 High-order finite difference and finite volume WENO schemes and discontinuous Galerkin methods for CFD. Int. J. Comput. Fluid Dyn. 17, $107-118$.

Shu, C W \& Osher, S 1988 Efficient implementation of essentially nonoscillatory shock-capturing schemes. J. Comput. Phys. 77, 439-471.

Shu, C W \& Osher, S 1989 Efficient implementation of essentially nonoscillatory shock-capturing schemes, II. J. Comput. Phys. 83, 32-78.

Shyue, K M 1998 An efficient shock-capturing algorithm for compressible multicomponent problems. J. Comput. Phys. 142, 208-242.

Shyue, K M 2001 A fluid-mixture type algorithm for compressible multicomponent flow with Mie-Grüneisen equation of state. J. Comput. Phys. 171, 678-707.

SoD, G A 1978 A survey of several finite difference methods for systems of nonlinear hyperbolic conservation laws. J. Comput. Phys. 27, 1-31.

SusLICK, K S 1990 Sonochemistry. Science 247, 1439-1445. 
Taleyarkhan, R P, West, C D, Cho, J S \& Lahey Jr., R T 2002 Evidence for nuclear emissions during acoustic cavitation. Science 295, 1868-1873.

TAnguay, M 2004 Computation of bubbly cavitating flow in shock wave lithotripsy. PhD thesis, California Institute of Technology.

Thompson, K W 1987 Time dependent boundary conditions for hyperbolic systems. J. Comput. Phys. 68, 1-24.

Thompson, K W 1990 Time-dependent boundary conditions for hyperbolic systems, II. J. Comput. Phys. 89, 439-461.

Thompson, P A 1984 Compressible-Fluid Dynamics. Maple Press Company.

Thornycroft, J \& Barnaby, S W 1895 Torpedo boat destroyers. Proc. Inst. of Civ. Engrs 122, 51-103.

Titarev, V A \& Toro, E F 2004 Finite-volume WENO schemes for threedimensional conservation laws. J. Comput. Phys. 201, 238-260.

Tomita, Y \& Shima, A 1986 Mechanisms of impulsive pressure generation and damage pit formation by bubble collapse. J. Fluid Mech. 169, 535-564.

Tomita, Y, Shima, A \& Takahashi, K 1983 The collapse of a gas bubble attached to a solid wall by a shock wave and the induced impact pressure. Trans. ASME I: J. Fluids Engng 105, 341-349.

Toro, E F 1999 Riemann Solvers and Numerical Methods for Fluid Dynamics. Springer-Verlag.

Toro, E F, Spruce, M \& Speares, W 1994 Restoration of the contact surface in the HLL-Riemann solver. Shock Waves 4, 25-34.

Tryggvason, G, Bunner, B, Esmaeeli, A, Juric, D, Al-Rawahi, N, Tauber, W, Han, J, NAs, S \& Jan, Y J 2001 A front-tracking method for the computations of multiphase flow. J. Comput. Phys. 169, 708-759. 
Tulin, M P 1969 On the creation of ultra-jets. In L. I. Sedov 60th Anniversary Volume: Problems of Hydrodynamics and Continuum Mechanics. Soc. for Ind. and Ind. Appl. Maths.

Versluis, M, Schmitz, B, von Der Heydt, A \& Lohse, D 2000 How snapping shrimp snap: through cavitating bubbles. Science 289, 2114-2117.

Vogel, A, Lauterborn, W \& Timm, R 1988 Acoustic transient generation by laser-produced cavitation bubbles near solid boundaries. J. Acoust. Soc. Am. 84, 719-731.

Vogel, A, Lauterborn, W \& Timm, R 1989 Optical and acoustic investigations of the dynamics of laser-produced cavitation bubbles near a solid boundary. $J$. Fluid Mech. 206, 299-338.

Wackers, J \& Koren, B 2005 A fully conservative model for compressible twofluid flow. Int. J. Numer. Meth. Fluids 47, 1337-1343.

Walmsley, A D, Laird, W R E \& Williams, A R 1988 Dental plaque removal by cavitational activity during ultrasonic scaling. J. Clin. Periodontal. 15, 539543.

Walters, J K \& Davidson, J F 1962 The initial motion of a gas bubble formed in an inviscid liquid. Part 1. The two-dimensional bubble. J. Fluid Mech. 12, 408-416.

Ward, B \& Emmony, D C 1991 Direct observation of the pressure developed in a liquid during cavitation-bubble collapse. Appl. Phys. Lett. 59, 2228-2230.

Wardlaw, A B \& Luton, J A 2000 Fluid-structure interaction mechanisms for close-in explosions. Shock Vib. 7, 265-275.

Willis, L R, Evan, A P, Connors, B A, Shao, Y, Blomgren, P M, Pratt, J H, Fineberg, N S \& Lingeman, J E 2005 Shockwave lithotripsy: doserelated effects on renal structure hemodynamics, and tubular function. J. Endourol. 19, 90-101. 
XI, X \& Zhong, P 2001 Dynamics photoelastic study of the transient stress field in solids during shock wave lithotripsy. J. Acoust. Soc. Am. 109, 1226-1239.

YANG, H 1990 An artificial compression method for ENO schemes: the slope modification method. J. Comput. Phys. 89, 125-160.

Yoganathan, A P, He, Z \& Jones, S C 2004 Fluid mechanics of heart valves. Annu. Rev. Biomed. Eng. 6, 331-362.

Zhang, S, Duncan, J H \& Chahine, G L 1993 The final stage of the collapse of a cavitation bubble near a rigid wall. J. Fluid Mech. 257, 147-181.

Zhong, P, Chuong, C J \& Preminger, G M 1993 Propagation of shock waves in elastic solids caused by cavitation microjet impact. II: Application in extracorporeal shock wave lithotripsy. J. Acoust. Soc. Am. 94, 29-36.

Zhu, S, Cocks, F H, Preminger, G M \& Zhong, P 2002 The role of stress waves and cavitation in stone comminution in shock wave lithotripsy. Ultrasound Med. Biol. 28, 661-671. 